\title{
The Determination and Evaluation of Pyrolytic Products: A Focus on Synthetic Cannabinoids
}

\author{
Stephen Raso
}

Follow this and additional works at: https://researchrepository.wvu.edu/etd

\section{Recommended Citation}

Raso, Stephen, "The Determination and Evaluation of Pyrolytic Products: A Focus on Synthetic Cannabinoids" (2018). Graduate Theses, Dissertations, and Problem Reports. 6481.

https://researchrepository.wvu.edu/etd/6481

This Dissertation is protected by copyright and/or related rights. It has been brought to you by the The Research Repository @ WVU with permission from the rights-holder(s). You are free to use this Dissertation in any way that is permitted by the copyright and related rights legislation that applies to your use. For other uses you must obtain permission from the rights-holder(s) directly, unless additional rights are indicated by a Creative Commons license in the record and/ or on the work itself. This Dissertation has been accepted for inclusion in WVU Graduate Theses, Dissertations, and Problem Reports collection by an authorized administrator of The Research Repository @ WVU.

For more information, please contact researchrepository@mail.wvu.edu. 


\title{
The Determination and Evaluation of Pyrolytic Products: A Focus on Synthetic Cannabinoids
}

\author{
Stephen Raso
}

\author{
Dissertation submitted to the \\ Eberly College of Arts and Sciences \\ at West Virginia University \\ in partial fulfillment of the requirements for the degree of \\ Doctor of Philosophy in Chemistry
}

Suzanne Bell, Ph.D., Chair

Jonathan Boyd, Ph.D.

Harry Finklea, Ph.D.

Glen Jackson, Ph.D.

Patrick Callery, Ph.D.

C. Eugene Bennett Department of Chemistry

\section{Morgantown, West Virginia \\ 2018}

Key Words: synthetic cannabinoids, pyrolysis, thermal degradation, gas chromatograph mass spectrometry, liquid chromatography mass spectrometry 


\section{Abstract \\ The Determination and Evaluation of Pyrolytic Products: A Focus on Synthetic Cannabinoids}

\section{Stephen Raso}

Synthetic cannabinoids have become a ubiquitous challenge in forensic toxicology and seized drug analysis. Acute toxic effects associated with these drugs include tachycardia, seizures, depression, possible suicidal tendencies, and the onset of psychotic episodes. Synthetic cannabinoids were initially synthesized for research purposes in understanding the receptorligand interactions at the $\mathrm{CB}_{1}$ and $\mathrm{CB}_{2}$ cannabinoid receptors. They are similar to $\Delta^{9}$ tetrahydrocannabinol (THC) and also act as agonists at the cannabinoid receptors. Originally it was hoped that this class of drug would be useful for pain relief, but instead this class of compounds has become widely abused, typically via smoking or heated vapor inhalation. The mode of ingestion is important in this context as heating creates many new potentially toxic agents, some of which may interact with the cannabinoid receptors as well.

The objectives of this research are to determine pyrolytic products produced from the smoking process of selected representative synthetic cannabinoids and evaluate their presence in true case samples to establish the necessity for inclusion in toxicological assessment. This is a timely project given that current literature reports describe the detection of thermal degradation products of two synthetic cannabinoids in traditional toxicological matrices; blood and urine. Fundamental understanding of the toxic effects of thermal degradation products could assist forensic toxicologists in assessing intoxication and in development of new assays. This knowledge could also be of use to the field of medicolegal death investigation and finally in the broader context of public health and safety.

Proof of thermal degradation product production and ingestion are crucial to set a precedent for further studies of possible toxicity. The current research utilizes an optimized pyrolysis methodology, where a predictive model for thermal degradation was established. An extraction and LC/MS/MS method was validated according to the guidelines set forth by the Standard Practices for Method Validation in Forensic Toxicology, and the project aids in achieving research goals on the set out by SWGTOX in August 2014; such as goal 3.1, Characterization of Toxicants and 3.2 Factors Affecting the Interpretation of Forensic Toxicology Data. 


\section{Acknowledgements}

I would like to acknowledge the National Institute of Justice, as they awarded me with a graduate research fellowship that allowed me to conduct this research with full funding. This award has been extremely beneficial in purchasing all pertinent supplies and traveling to multiple national conferences.

A second acknowledgement that is necessary is Dr. Barry Logan and the National Medical Services Labs in Willow Grove, PA. They graciously allowed me to visit their facility and brief them on the research I was performing. In turn, they agreed to supply me with case samples or post mortem blood. An important piece of the project could not have been done without their collaboration. 


\section{Table of Contents}

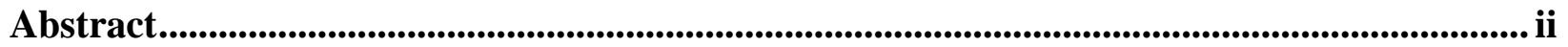

Acknowledgements ................................................................................................................................ iii

List of Figures.............................................................................................................................................. vii

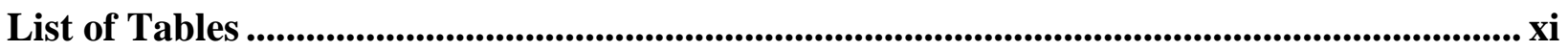

List of Abbreviations, Symbols and Nomenclature ......................................................................... xii

Chapter 1: Introduction ......................................................................................................... 1

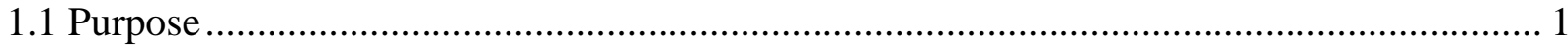

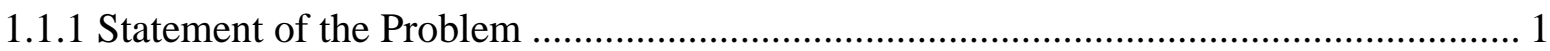

1.1.2 Review of Research Literature .............................................................................. 4

1.1.3 Goals and Research Design ......................................................................................... 9

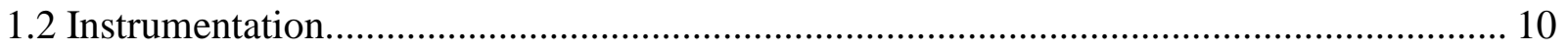

1.2.1 Gas Chromatography - Mass Spectrometry (GC/MS) ................................................. 10

1.2.2 Liquid Chromatography - Tandem Mass Spectrometry (LC/MS/MS) ........................ 12

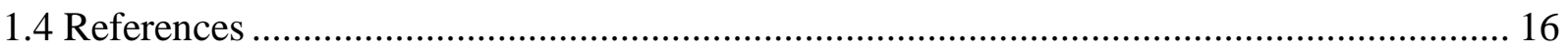

Chapter 2: Qualitative Analysis and Detection of the Pyrolytic Products of JWH-018 and 11 Additional Synthetic Cannabinoids in the Presence of Common Herbal Smoking Substrates................................................................................................................................................. 23

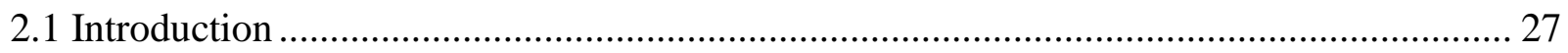

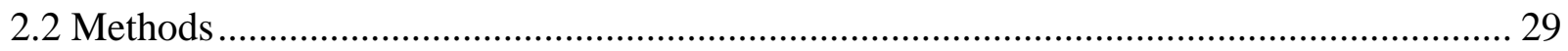

2.2.1 Materials and Reagents..................................................................................... 29

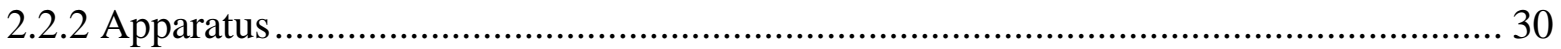

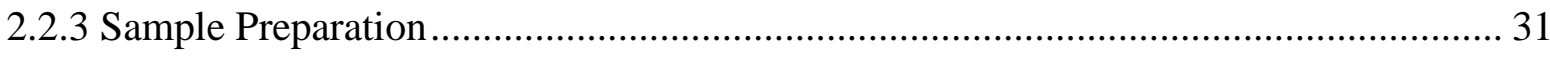

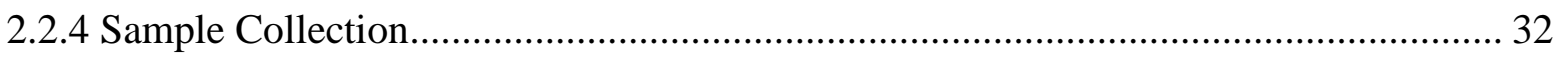

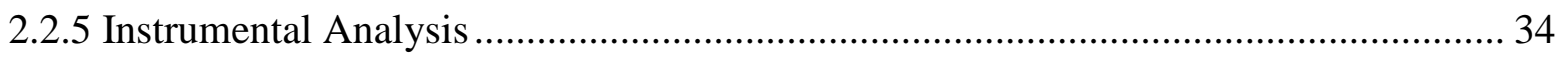

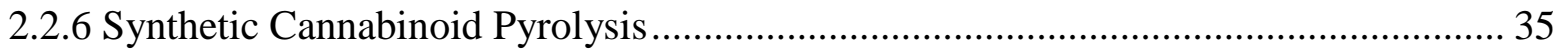

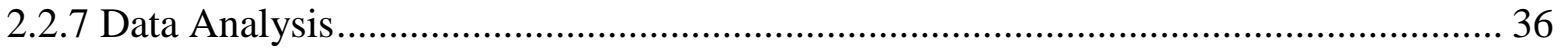

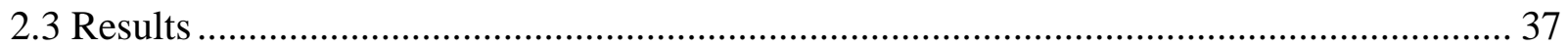

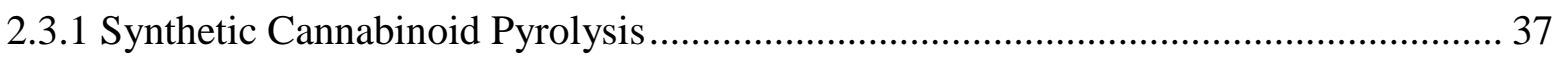

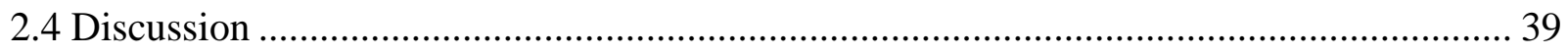

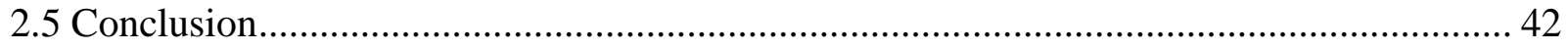




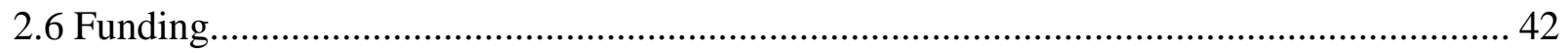

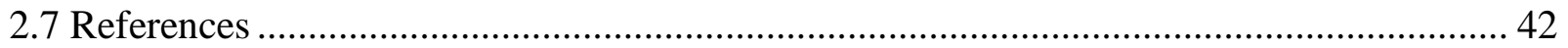

Chapter 3: Utilization of Novel Pyrolysis Methodology to Evaluate the Thermal Degradation Products of Classical Drugs of Abuse .......................................................................... 46

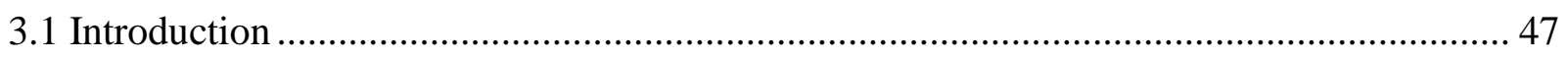

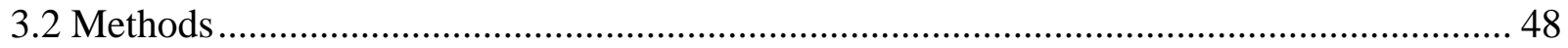

3.2.1 Materials and Reagents........................................................................................ 48

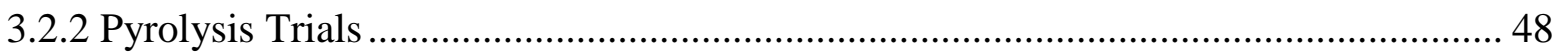

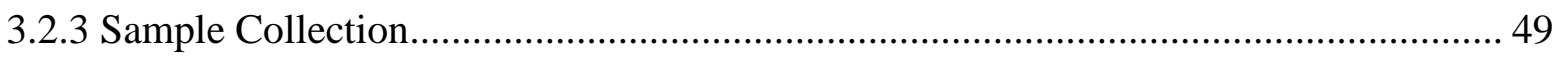

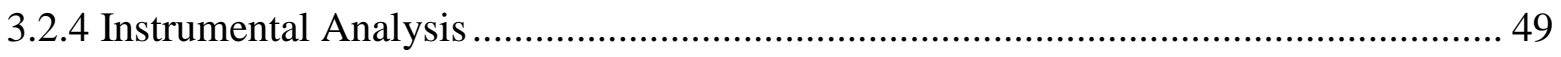

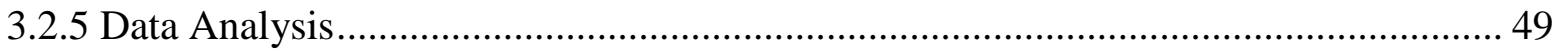

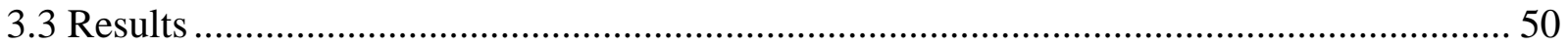

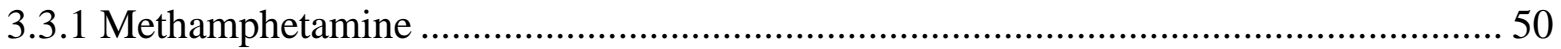

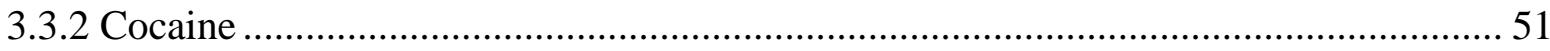

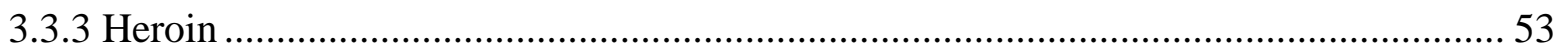

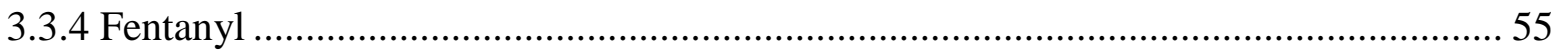

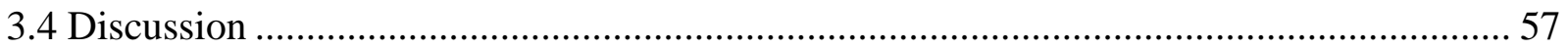

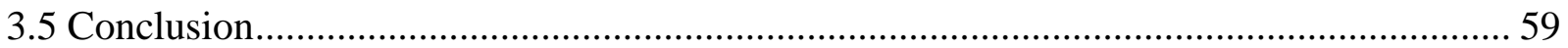

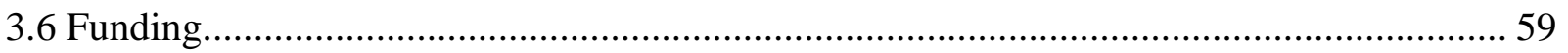

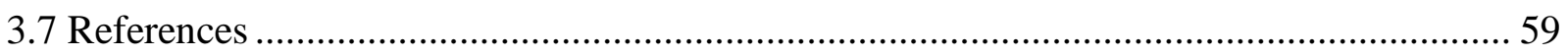

Chapter 4: Detection of Confirmed Thermal Degradation Products of Ten Synthetic Cannabinoids in Post-Mortem Blood Samples .................................................................................... 63

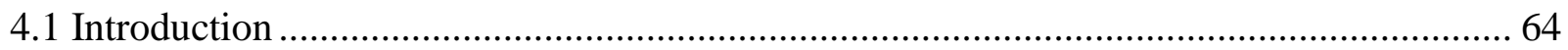

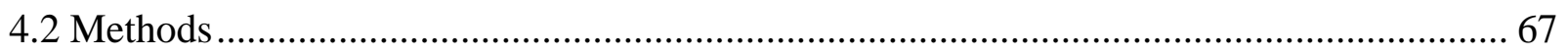

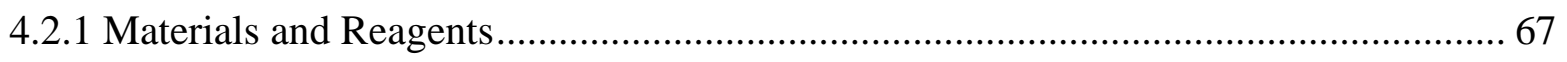

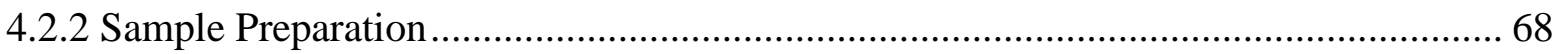

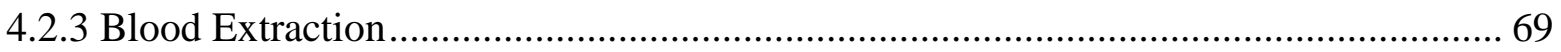

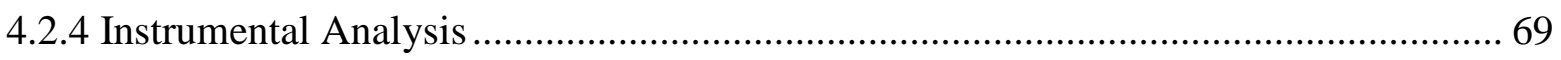

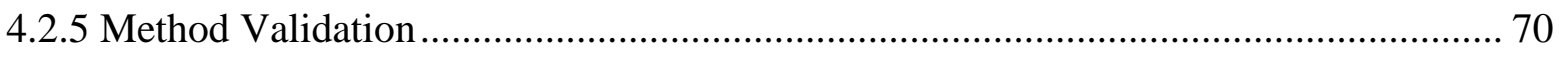

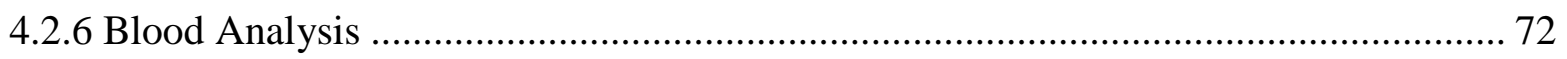

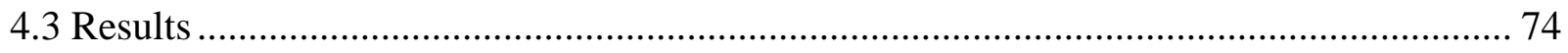

4.3.1 Method Validation.................................................................................................. 74

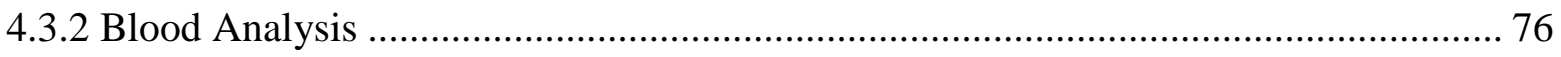




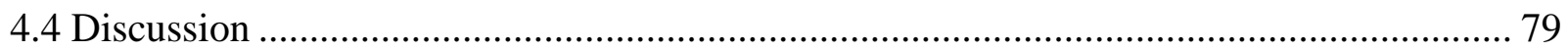

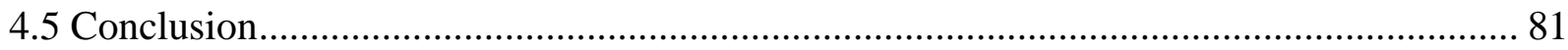

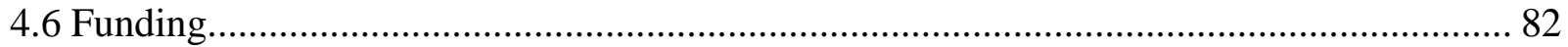

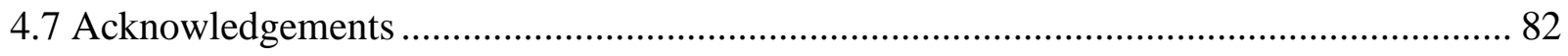

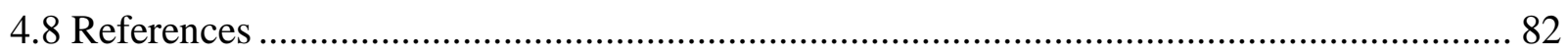

Chapter 5: Conclusions and Future Directions.......................................................... 85

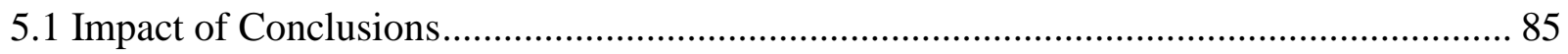

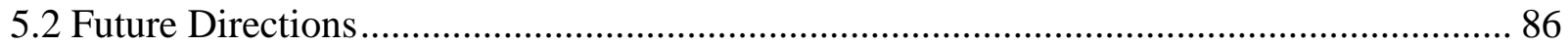

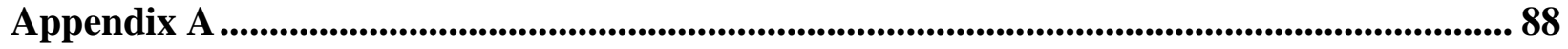

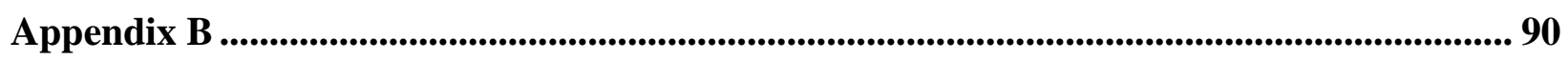

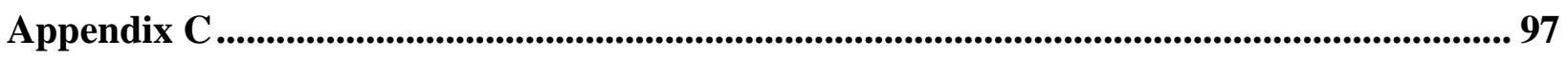

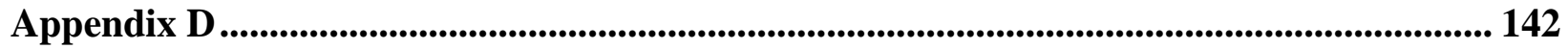

Appendix E .................................................................................................................................. 145 


\section{List of Figures}

Figure 1.1 - Schematic of a gas-chromatograph - mass spectrometer........................ 11

Figure 1.2 - Schematic of a liquid chromatograph........................................ 13

Figure 1.3 - Schematic of an LC injection valve........................................ 14

Figure 1.4 - Schematic of a triple quadrupole mass spectrometer.......................... 15

Figure 2.1 - A reproduced MS spectrum of predicted product, N-(1,2-dimethylpropyl)-4-

cinnolinamine, along with its predicted breakdown...................................... 32

Figure 2.2 - Breakdown chart of JWH-018 to it proposed observed pyrolytic products; (*)

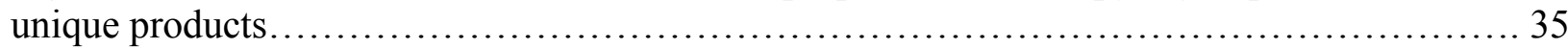

Figure 2.3 - Chart displaying each parent synthetic cannabinoid that produced quinoline...... 40

Figure 3.1 - Proposed pyrolytic breakdown of methamphetamine into five pyrolytic

products. $\mathrm{M}=$ Methamphetamine metabolite; $\mathrm{P}=$ Previously found pyrolytic.................. 51

Figure 3.2 - Proposed pyrolytic breakdown of cocaine into eight pyrolytic products. $\mathrm{M}=$

Cocaine metabolite; $\mathrm{P}=$ Previously found pyrolytic; $\mathrm{M}^{*}=$ Metabolite of a previously

reported cocaine pyrolytic product................................................... 52

Figure 3.3 - Proposed pyrolytic breakdown of heroin into eight pyrolytic products. $\mathrm{M}=$

Heroin metabolite; $\mathrm{P}=$ Previously found pyrolytic; $\mathrm{M}^{\wedge}=$ Metabolite of a newly reported pyrolytic product of heroin.......................................................... 54

Figure 3.4 - Proposed breakdown of N,3-O,6-O-triacetylnormorphine, compound 2 from

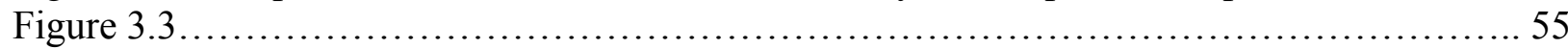

Figure 3.5 - Proposed pyrolytic breakdown of fentanyl into six pyrolytic products. $\mathrm{M}=$

Fentanyl metabolite; $\mathrm{P}=$ Previously found pyrolytic..................................... 56

Figure 4.1 - The structure of serotonin and examples of proposed synthetic cannabinoid

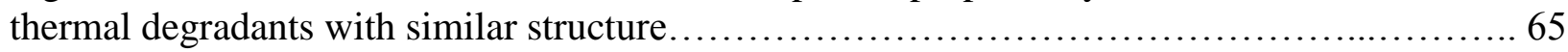

Figure 4.2 - The structure of each parent synthetic cannabinoid contained in the 23 case

blood samples...................................................................... 74

Figure 4.3 - The structure of the 7 consistently observed thermal degradation products........ 78

Figure B1 - Photograph of the in-house built apparatus................................... 90

Figure B2 - Schematic of the quartz tubing T-junction where the pyrolysis was contained...... 91

Figure B3 - Schematic of the bored Teflon plug used to hold the pyrolysis apparatus parts

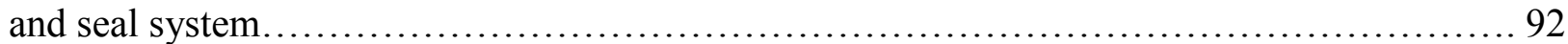

Figure B4 - The heating signature data of the propane torch with no herbal material present....92

Figure B5 - Heating signature of the reaction zone with each herbal material present, and

the average of all trials indicated by the black dashed line................................... 93

Figure B6 - Schematic of the exhaustive sampling of JWH-018 on Damiana to optimize

the number of washes and replicates................................................... 95

Figure C1 - Plot of volume added vs number of sprays with the equation of best fit line

showing the volume per spray as the slope............................................... 100

Figure $\mathrm{C} 2$ - Proposed mechanism of a pyrolytic cleavage at the nitrogen within the indole

ring (or could be an indazole).......................................................... 107

Figure C 3 - Proposed mechanism of a pyrolytic cleavage of the central carbonyl group

on the non-indole/indazole side .........................................................108

Figure C4 - Proposed mechanism of a pyrolytic cleavage of the central carbonyl group

on the indole/indazole side ...............................................................

Figure C5 - Proposed mechanism of a ring expansion from indazole to cinnoline ..............109 
Figure C6 - Thermal degradation breakdown of AB-CHMINACA to its proposed observed pyrolytic products ..................................................... 110 Figure C7 - Thermal degradation breakdown of AB-FUBINACA to its proposed observed pyrolytic products.

Figure $\mathrm{C} 8$ - Thermal degradation breakdown of AB-PINACA to its proposed observed

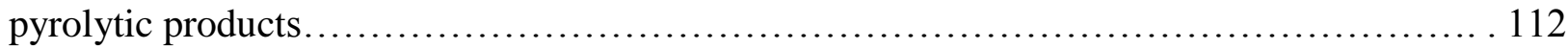

Figure C9 - Thermal degradation breakdown of AM-694 to its proposed observed pyrolytic products ............................................................... 113

Figure C10 - Thermal degradation breakdown of AM-2201 to its proposed observed pyrolytic products.............................................................. 114

Figure C11 - Thermal degradation breakdown of JWH-018 to its proposed observed pyrolytic products............................................................... 115

Figure C12 - Thermal degradation breakdown of JWH-073 to its proposed observed pyrolytic products......................................................... 116

Figure C13 - Thermal degradation breakdown of JWH-081 to its proposed observed pyrolytic products............................................................. 117

Figure C14 - Thermal degradation breakdown of JWH-210 to its proposed observed pyrolytic products 118

Figure C15 - Thermal degradation breakdown of MAM-2201 to its proposed observed pyrolytic products

Figure C16 - Thermal degradation breakdown of UR-144 to its proposed observed pyrolytic products

Figure C17 - Thermal degradation breakdown of XLR-11 to its proposed observed pyrolytic products.

Figure C18 - Breakdown of parent synthetic cannabinoids that produced the proposed thermal degradant of (1H-indo-3-yl)-(2,2,3,3-tetramethylcyclopropyl)-Methanone. Figure C19 - Breakdown of parent synthetic cannabinoids that produced the proposed thermal degradant of $1 \mathrm{H}$-Indazole-3-carboxaldehyde.

Figure C20 - Breakdown of parent synthetic cannabinoids that produced the proposed thermal degradant of 1-Methylindazole.

Figure C21 - Breakdown of parent synthetic cannabinoids that produced the proposed thermal degradant of 1-Methylindazole-3-formamide.

Figure C22 - Breakdown of parent synthetic cannabinoids that produced the proposed thermal degradant of 1-pentyl-3-Acetylindole.

Figure C23 - Breakdown of parent synthetic cannabinoids that produced the proposed thermal degradant of 1-Pentylindole.

Figure C24 - Breakdown of parent synthetic cannabinoids that produced the proposed thermal degradant of 1-Pentylindole-3-carboxaldehyde.

Figure C25 - Breakdown of parent synthetic cannabinoids that produced the proposed thermal degradant of 1-Pentylisatin

Figure C26 - Breakdown of parent synthetic cannabinoids that produced the proposed thermal degradant of 3-Naphthoylindole.

Figure C27 - Breakdown of parent synthetic cannabinoids that produced the proposed thermal degradant of 4-Hydroxycinnoline or 4-Cinnolinamine.

Figure C28 - Breakdown of parent synthetic cannabinoids that produced the proposed thermal degradant of Formylvaline amide. 
Figure C29 - Breakdown of parent synthetic cannabinoids that produced the proposed thermal degradant of Hydroxyquinoline............................................. 133 Figure C30 - Breakdown of parent synthetic cannabinoids that produced the proposed thermal degradant of Indole.

Figure C31 - Breakdown of parent synthetic cannabinoids that produced the proposed

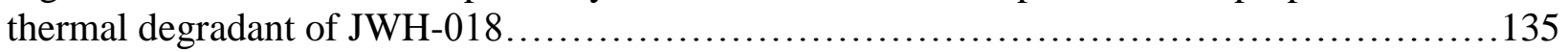

Figure C32 - Breakdown of parent synthetic cannabinoids that produced the proposed thermal degradant of Methylindole.

Figure C33 - Breakdown of parent synthetic cannabinoids that produced the proposed thermal degradant of $\mathrm{N}$-(2-methylpropyl)-4-Cinnolinamine....

Figure C34 - Breakdown of parent synthetic cannabinoids that produced the proposed thermal degradant of Naphthalene.................................................... 138 Figure C35 - Breakdown of parent synthetic cannabinoids that produced the proposed thermal degradant of N-methyl-3-Naphthoylindole.

Figure C36 - Breakdown of parent synthetic cannabinoids that produced the proposed thermal degradant of N-phenyl-1-Naphthamide.

Figure C37 - Breakdown of parent synthetic cannabinoids that produced the proposed thermal degradant of Quinoline.

Figure E1 - Possible thermal degradation products for the parent synthetic cannabinoid,

$5 \mathrm{~F}-\mathrm{ADB}$.

Figure E2 - Possible thermal degradation products for the parent synthetic cannabinoid,

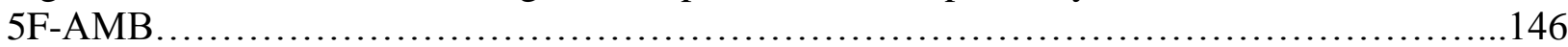

Figure E3 - Possible thermal degradation products for the parent synthetic cannabinoid, 5F-PB-22 .............................................................. 147

Figure E4 - Possible thermal degradation products for the parent synthetic cannabinoid,

AB-CHMINACA ............................................................. 148

Figure E5 - Possible thermal degradation products for the parent synthetic cannabinoid, AB-FUBINACA............................................................. 149

Figure E6 - Possible thermal degradation products for the parent synthetic cannabinoid, AB-PINACA .............................................................. 150

Figure E7 - Possible thermal degradation products for the parent synthetic cannabinoid, ADB-CHMINACA (MAB-CHMINACA) ....................................... 151

Figure E8 - Possible thermal degradation products for the parent synthetic cannabinoid,

ADB-FUBINACA ......................................................... 152

Figure E9 - Possible thermal degradation products for the parent synthetic cannabinoid,

FUB-AKB-48.......................................................... 153

Figure E10 - Possible thermal degradation products for the parent synthetic cannabinoid, MMB-FUBINACA........................................................... 154

Figure E11 - Linearity plot of concentration $(1-100 \mathrm{ng} / \mathrm{ml})$ vs intensity for 5F-ADB.......155

Figure E12 - Linearity plot of concentration $(0.1-100 \mathrm{ng} / \mathrm{ml})$ vs intensity for 5F-AMB .....155

Figure E13 - Linearity plot of concentration $(1-100 \mathrm{ng} / \mathrm{ml}) \mathrm{vs}$ intensity for 5F-PB-22......156

Figure E14 - Linearity plot of concentration $(1-100 \mathrm{ng} / \mathrm{ml})$ vs intensity for

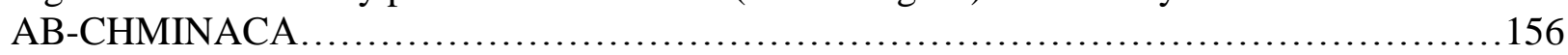

Figure E15 - Linearity plot of concentration $(1-100 \mathrm{ng} / \mathrm{ml})$ vs intensity for

AB-FUBINACA. 
Figure E16 - Linearity plot of concentration $(1-100 \mathrm{ng} / \mathrm{ml})$ vs intensity for

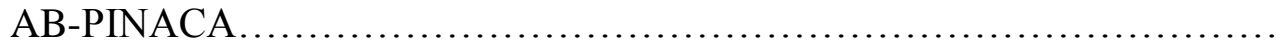

Figure E17 - Linearity plot of concentration $(1-100 \mathrm{ng} / \mathrm{ml})$ vs intensity for

ADB-FUBINACA...

Figure E18 - Linearity plot of concentration $(1-100 \mathrm{ng} / \mathrm{ml})$ vs intensity for FUB-AKB-48 158

Figure E19 - Linearity plot of concentration $(1-100 \mathrm{ng} / \mathrm{ml}) \mathrm{vs}$ intensity for

MAB-CHMINACA.

Figure E20 - Linearity plot of concentration $(1-100 \mathrm{ng} / \mathrm{ml})$ vs intensity for

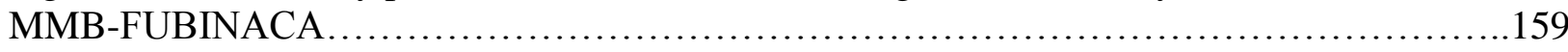

Figure E21 - LC chromatogram of a mixture of all 10 synthetic cannabinoids..................160

Figure E22 - LC chromatogram of a standard of 4-Hydroxycinnoline ........................160

Figure E23 - LC chromatogram of a standard of 1H-Indazole-3-carboxaldehyde..............160

Figure E24 - LC chromatogram of a standard of Cinnoline................................ 161

Figure E25 - LC chromatogram of a standard of 1-Methylindazole......................... 161

Figure E26 - LC chromatogram of a standard of Indazole............................... 161

Figure E27 - LC chromatogram of a standard of 1-Pentylindazole.........................162

Figure E28 - Example LC chromatogram of a blood sample containing indazole...............162

Figure E29 - Example LC chromatogram of a blood sample containing 1-pentylindazole......163

Figure E30 - Example LC chromatogram of a blood sample containing 4-methylcinnoline... 163 


\section{List of Tables}

Table 2.1 - Overview of the identification method for the thermal degradation products observed in the chapter 2 manuscript. If not indicated as confirmed, it is a tentative identification.

Table 2.2 - Proposed observed pyrolytic products unique to their respectively shown synthetic cannabinoid.

Table 2.3 - Proposed yrolytic products with the parent synthetic cannabinoids they are commonly produced from; a)AB-CHMINACA b)AB-FUBINACA c)AB-PINACA d)AM-694 e)AM-2201 f)JWH-018 g)JWH-073 h)JWH-081 i)JWH-210 j)MAM-2201 k)UR-144 1)XLR-11.

Table 4.1 - Previously detected synthetic cannabinoids within the case blood samples obtained and reported from NMS Labs.

Table 4.2 - Observed thermal degradation products from the case blood samples. 1: 4Hydroxycinnoline; 2: 1H-Indazole-3-carboxaldehyde; 3: Indazole; 4: Cinnoline; 5: NMethylindazole; 6: 4-Methylcinnoline; 7: N-Pentylindazole. * - Not Confirmed................77

Table B1 - Proposed identifications of products observed in herbal material pyrolysis.

Table C1 - Data for method 1 of the determination of volume per spray of the sprayer used for sample preparation. * : Density of water used was $0.9982 \mathrm{~g} / \mathrm{ml}$.

Table C2 - The mass data for method 2 of the determination of the volume per spray..........99

Table C3 - Data for determining the loss of drug compound due to evaporation only..........101

Table C4 - Data for the determination of loss due to the spraying of drug solution onto the herbal matrix by periodic sprays.

Table C5 - Data for the determination of loss due to the spraying of drug solution onto the herbal matrix all at once.

Table C6 - Data for the determination of recovery of overall sample preparation, pyrolysis and sample collection process.

Table D1 - Compound dependent parameters for the synthetic cannabinoids and observed pyrolytic products. * - predicted transition ions and parameters kept the same as cinnoline as the compounds differ by only a single methyl group and a reference standard was unavailable.

Table D2 - Linearity, LOD and LOQ values determined for each synthetic cannabinoid.......143

Table D3 - Precision and Accuracy data for both within-day and between-day evaluations....144

Table D4 - Average extraction recoveries and matric effects; analyzed at $n=3$. . . 


\section{List of Abbreviations, Symbols and Nomenclature}

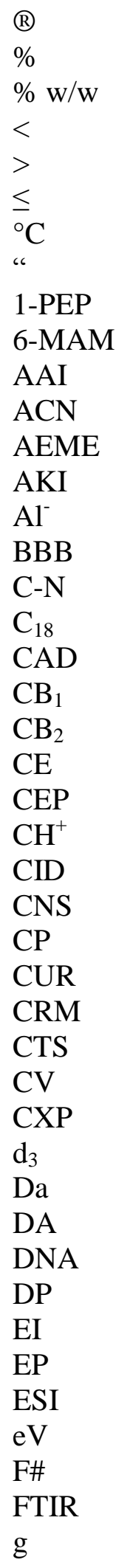

Registered

Percent

Percent by Weight

Less Than

Greater Than

Less Than or Equal To

Degrees Celsius

Inches

1-Phenylpyridinium

6-Monoacetylmorphine

Aminoalkylindole

Acetonitrile

Anhydroecgonine methyl ester

Acute Kidney Injury

Aluminum Anion

Blood Brain Barrier

Carbon-Nitrogen Bond

Carbon-18 Chain

Collision Gas

Cannabinoid Receptor 1

Cannabinoid Receptor 2

Collision Energy

Collision Cell Entrance Potential

Carbon Hydrogen Cation

Collision Induced Dissociation

Central Nervous System

Cyclohexylphenol

Curtain Gas

Charge Residue Model

Counts

Coefficient of Variance

Collision Cell Exit Potential

Deuterated Hydrogen

Dalton

Dopamine

Deoxyribonucleic Acid

Declustering Potential

Electron Impact Ionization

Entrance Potential

Electrospray Ionization

Electron Volt

Flask Sample Number

Fourier-Transfer Infrared Spectroscopy

Gram 


\begin{tabular}{|c|c|}
\hline $\mathrm{GC}$ & Gas Chromatograph \\
\hline GC/MS & Gas Chromatography-Mass Spectrometry \\
\hline \multirow[t]{2}{*}{ GC/MS/MS } & Gas Chromatography-Tandem Mass \\
\hline & Spectrometry \\
\hline GHS & Globally Harmonized System \\
\hline GS1 & Gas 1 \\
\hline GS2 & Gas 2 \\
\hline HPLC & High Pressure Liquid Chromatography \\
\hline \multirow[t]{2}{*}{ HSL } & Heroin induced spongiform \\
\hline & leukoencephalopathy \\
\hline $\mathrm{HU}$ & Hebrew University \\
\hline I & Inner Diameter \\
\hline IEM & Ion Evaporation Model \\
\hline Inc. & Incorporated \\
\hline inHg & Inches of Mercury \\
\hline IS & Ionization Spray \\
\hline K-type & Thermocouple Wire: Graded -270 - 1260C \\
\hline $\mathrm{kPa}$ & Kilopascal \\
\hline $\mathrm{LC}$ & Liquid Chromatography \\
\hline \multirow[t]{2}{*}{$\mathrm{LC} / \mathrm{MS} / \mathrm{MS}$} & Liquid Chromatography-Tandem Mass \\
\hline & Spectrometry \\
\hline LC-QTOF-MS & $\begin{array}{l}\text { Liquid Chromatography-Quadrupole Time } \\
\text { of Flight-Mass Spectrometry }\end{array}$ \\
\hline LOD & Limit of Detection \\
\hline LOQ & Limit of Quantitation \\
\hline $\mathrm{m}$ & Meter \\
\hline M & Molarity \\
\hline $\mathrm{m} / \mathrm{z}$ & Mass to Charge Ratio \\
\hline $\mathrm{MeOH}$ & Methanol \\
\hline $\mathrm{mg}$ & Milligram \\
\hline $\min$ & Minute \\
\hline $\mathrm{ml}$ & Milliliter \\
\hline $\mathrm{mm}$ & Millimeter \\
\hline $\mathrm{mmHg}$ & Millimeter of Mercury \\
\hline MRM & Multiple Reaction Monitoring \\
\hline MS & Mass Spectrometry/Mass Spectrum \\
\hline NE & Norepinephrine \\
\hline $\mathrm{NIH}$ & National Institute of Health \\
\hline NIST & National Institute of Standards and \\
\hline & Technology \\
\hline $\mathrm{nM}$ & Nanomolar \\
\hline NMR & Nuclear Magnetic Resonance \\
\hline NPS & Novel Psychoactive Substance \\
\hline NMS & National Medical Services \\
\hline $\mathrm{O}$ & Outer Diameter \\
\hline P-SA\# & Plant-Spray All Sample Number \\
\hline
\end{tabular}




$\begin{array}{ll}\text { P-SP\# } & \text { Plant-Spray Periodic Sample Number } \\ \text { PCP } & \text { Phencyclidine } \\ \text { pH } & \text { Potential of Hydrogen } \\ \text { psi } & \text { Pound per Square Inch } \\ \text { QW\# } & \text { Quartz Wool Sample Number } \\ \text { R }^{2} & \text { Coefficient of Determination } \\ \text { RCS } & \text { Research Supply Company } \\ \text { RF } & \text { Radio Frequency } \\ \text { RI } & \text { Retention Index } \\ \text { s or sec } & \text { Seconds } \\ \text { SV\# } & \text { Spray into Vial Sample Number } \\ \text { SWGTOX } & \text { Scientific Working Group for Toxicology } \\ \text { T\# } & \text { Tube Sample Number } \\ \text { THC } & \Delta^{9} \text {-Tetrahydrocannabinol } \\ \text { TM } & \text { Trademark } \\ \mu l & \text { Microliter } \\ \mu \mathrm{m} & \text { Micrometer } \\ \mu \mathrm{M} & \text { Micromolar } \\ \text { V } & \text { Voltage } \\ \text { W } & \text { Wall Width } \\ \text { WHO } & \text { World Health Organization } \\ \text { WIN } & \text { Sterling Winthrop } \\ & \end{array}$




\section{Chapter 1: Introduction}

\subsection{Purpose}

\subsubsection{Statement of the Problem}

In the past few years, notable progress has been made in identifying the metabolites of synthetic cannabinoids but there are two areas that remain relatively unexplored: 1) the toxicity and mechanisms of toxicity of these drugs and their metabolites; and identification of common pyrolytic products and their toxicity ${ }^{1-10}$. The latter is critical given that the most common mode of ingestion of the synthetic cannabinoids is smoking or heated vapor generation. Of particular concern to forensic toxicology, medicolegal death investigation, and public health and safety are the acute toxicities of these compounds. The goal of this research is to provide foundational knowledge to these communities and to provide a framework for developing new diagnostic and analytical tools for use in the context.

Synthetic cannabinoid products act on the same receptors as $\Delta^{9}$-tetrahydrocannabinol, the active ingredient within cannabis; however, they produce acute toxic responses that are not observed with the use of $\mathrm{THC}^{11-16}$. The side effects are considered the "cannabinoid tetrad", which includes (1) hypothermia, (2) analgesia, (3) catalepsy and (4) suppression of locomotor activity $^{17-23}$. Toxic effects outside of the tetrad also are observed in the renal, cardiovascular, respiratory and nervous systems. The increased use of these products and the accompanying dangers to users led the Drug Enforcement Agency to begin temporarily scheduling compounds such as JWH-018, JWH-073, JWH-200, CP-47,497 and CP-47,497-C8 homologue as Schedule I compounds under the Controlled Substances Act. Since then, additional compounds have been added in over the years, and to date, there are 33 Schedule 1 synthetic cannabinoids ${ }^{15,20-21,24}$. 
Synthetic cannabinoids appeared in the mid-2000s primarily in Europe and soon thereafter in the United States ${ }^{11,15,25-27}$. In 2009, a number of countries in Europe and the United States analyzed seized so-called "Spice" products, and numerous synthetic cannabinoid products such as JWH-073, CP 47,497 and HU-210 among others were detected in these products ${ }^{11}$. In subsequent years, a large influx of synthetic cannabinoid products were introduced and became widely available which in turn led to an increased reporting by poison control centers and hospitals of synthetic cannabinoid ingestion ${ }^{26,28}$. In 2010, there were 2,906 suspected cases reported and 6,968 in 2011. The number decreased in $2013(2,668)$ and increased again in 2014 $(3,677)^{29}$. Driving while intoxicated, overdose, and death cases that are unknowingly related to synthetic cannabinoid ingestion may not be accurately reflected in these statistics.

The synthesis of THC analogs dates back to the 1960s when this family of drugs was first explored for use as pain relieving agents. Unanticipated acute toxic effects resulted in an end to this work and research attention turned to employing these compounds as research tools for understanding the structure of cannabinoid receptors and their receptor-ligand binding ${ }^{11,13,15,30}$. In the 1970's, Pfizer developed synthetic cannabinoids commonly termed cyclohexylphenols (CP), which are considered "non-classical" due to their lack of the benzopyran core of classical cannabinoids ${ }^{11,13}$. In the 1990's, John W. Huffman began synthesizing the aminolkylindole synthetic cannabinoids (AAI), which contain napthyl and indole groups. This family of compounds is identified by the JWH moniker ${ }^{11,13,26,31-32}$.

THC is an agonist for both the $\mathrm{CB}_{1}$ and $\mathrm{CB}_{2}$ cannabinoid receptors with roughly equivalent binding affinities for each. $\mathrm{THC}$ has a $\mathrm{CB}_{1}$ binding affinity of $41 \mathrm{nM}$, while early generation compounds JWH-018 and JWH-073 are 9nM and 8.9nM, respectively ${ }^{33} \cdot \mathrm{CB}_{1}$ receptors are located in the central nervous system, which produce the psychological effects 
observed in cannabis users ${ }^{11-12,14}$. Synthetic cannabinoids are structurally different than THC but still elicit similar psychoactive responses to $\mathrm{THC}^{11-14,16,22,34}$. They also bind to the cannabinoid receptors, and the binding affinity of common synthetic cannabinoids is higher than that of THC. The increased potency has been reported to cause increased dependence over time and possible chronic health risks ${ }^{35}$. The primary route of ingestion for these compounds is the inhalation of heated vapors. This immediate bioavailability through absorption in the lungs coupled with the higher binding affinity for the $\mathrm{CB}_{1}$ receptors and potency put users in risk of over dose, particularly naïve users ${ }^{36}$.

Recent work in forensic toxicology in the context of synthetic cannabinoids has focused on analytical assays. Methods have been published for detecting synthetic cannabinoids along with their respective metabolites in toxicological matrices using hyphenated chromatographic/ mass spectrometry instrumentation and immunoassays ${ }^{15,22,29,31,37-44}$. What is lacking from the current literature are reports and data describing these toxicants associated with the synthetic cannabinoids and associated pyrolytic products, their mechanisms of action, and how this information could be used to inform the interpretation of forensic toxicology data. In a 2012 publication, Wiebelhaus and Poklis et al. concluded that, "The increased potency of JWH-018 compared to THC, the variable amount of drug added to various herbal products, and unknown toxicity, undoubtedly contribute to public health risks of synthetic cannabinoids" ${ }^{45}$. Additionally, in a 2011 article published in Clinical Biochemistry, Penn and Langman et al. stated, "The compounds were also extracted by boiling rather than vaporization into smoke, which raises the question whether smoking would generate pyrolysis products different from the parent drug" 46 . Numerous pyrolytic products produced from the pyrolysis of the synthetic cannabinoid JWH-018 have been identified ${ }^{26}$. 
To the best of our knowledge, there has yet to be an extensive investigation into the ingestion and toxicity of the pyrolytic products produced when smoking synthetic cannabinoids. In a 2014 paper, Daw and Grabenauer et al. stated, "Synthetic cannabinoids degrade to other products when smoked. These pyrolysis products can be components that have an altered affinity to the $\mathrm{CB}_{1}$ and $\mathrm{CB}_{2}$ receptor or entities of unknown pharmacology and toxicology"47. There have been a few studies into the cell toxicity of synthetic cannabinoid parent compounds, and these can be used as a basis for advancing and expanding work into pyrolytic compounds as they are demonstrated to be ingested. Understanding the toxicity of the pyrolytic compounds could aid in filling the gap of toxicological knowledge regarding acute and chronic toxicity of synthetic cannabinoids and the associated issues related to forensic toxicology, medicolegal death investigation, and public health.

\subsubsection{Review of Research Literature}

One of the earliest studies that detected the presence of synthetic cannabinoids in an herbal incense product was in 2009, where the authors smoked a cigarette containing one of seven products they had collected ${ }^{25}$. Observations of the subjects were documented and several blood and urine samples were collected over time. The main synthetic cannabinoid detected in blood was the CP-47,497-C8 homologue. Subsequent analyses of the collected products, led to the discovery that JWH-018 was present in a number of the products. Pharm-THC, a licensed German company which produces and distributes Dronabinol®, went on record shortly after the report as saying they too had isolated JWH-018 from various seized Spice products ${ }^{11,28}$.

After these initial findings were publicized, a surge in research focusing on the analysis and detection of synthetic cannabinoids followed. In 2010, Teske et al detected JWH-018 in serum of two human subjects, but noted that after three hours, it was nearly undetectable by 
liquid chromatography tandem-mass spectrometry (LC-MS/MS ${ }^{48}$. Around the same time, two studies in Germany were conducted over a two year span to monitor the components found in herbal products. More than 140 samples were analyzed from June 2008 to September 2009 using GC-MS. Of the samples analyzed, 41 were found to contain no known synthetic cannabinoids, 28 were found to contain JWH-018, and 35 were found to contain JWH-073 ${ }^{49-50}$. Sobolevsky et al. were one of the first to report the detection of urinary metabolites of JWH-018 using gas chromatography tandem-mass spectrometry (GC-MS/MS) and LC-MS/MS ${ }^{1}$. Urine samples were collected and analyzed by police from three individuals who confessed to using an herbal incense product within 12 hours of being arrested. GC-MS/MS tentatively identified two metabolites, while LC-MS/MS revealed another 11 tentative metabolites. The authors noted that no detectable amount of parent drug was present in the urine samples.

The major metabolites of JWH-018 were determined in 2010 by in vitro phase I metabolism followed by Moller et al. reporting the findings from a study which screened approximately 7500 urine samples for JWH-018 and/or its metabolites for the purpose of human doping controls in $2011^{2,51}$. Subsequently, the World Anti-Doping Agency specifically added products containing the synthetic cannabinoid JWH-018, JWH-073, and HU-210 to their 2012 list of prohibited substances for athletes ${ }^{52}$.

In 2011, the group from Germany fully validated a LC-MS/MS method for the analysis of multiple synthetic cannabinoids, including JWH-018 and JWH-073. It was applied to approximately 100 serum samples provided from hospitals, police authorities, and rehabilitation centers, and the findings showed that after being banned by German legislation in 2009, JWH018 and JWH-073 were still found among products being sold. Of the 57 samples which tested positive for at least one synthetic cannabinoid, nine of them were positive for JWH-018, and six 
were positive for JWH- $073^{53}$. The validation of cannabinoid detection methods continues still, as in 2016 an LC/MS/MS method was reported that encompassed 32 synthetic cannabinoids ${ }^{54}$. Not long after, supplementary methods from both blood and urine specimens were published ${ }^{55-58}$.

Papers using GC-MS and LC-MS/MS were published by Grigoryev et al. which reported the detection of metabolites corresponding to JWH-250 as well as JWH-018 and JWH-073 in human serum, human urine, and rat urine $\mathrm{e}^{4-5}$. The human serum and urine samples were collected from arrested individuals that confessed to using herbal incense products and analyzed within 24 hours of arrest. A number of structures were tentatively identified as metabolites of the three synthetic cannabinoids, but no parent drug was present in detectable amounts, highlighting the need for assays which are capable of detecting the metabolites of these drugs ${ }^{4-5}$. Later in 2011 , the first LC-MS/MS studies using authentic standards to quantify the metabolites of JWH-018 and JWH-073 in human urine were published ${ }^{59-60}$. The more recent reports on newer generation cannabinoids shifted toward the use of hepatocytes for metabolic profiling, and the compounds analyzed included ADB-PINACA, 5F-ADB-PINACA, MAB-CHMINACA, MDMBFUBINACA, and ADB-FUBINACA ${ }^{61-63}$. An interesting report from 2016 allowed rats to inhale synthetic cannabinoids vapor and found that changes in dopamine and serotonin in brain microdialysates was observed, which could lead to neurotoxicity ${ }^{64}$.

The next step in research was to understand the binding affinity and activity of the synthetic cannabinoids. Brents et al. hypothesized that the seemingly unexplained side effects of synthetic cannabinoids were due in part to the metabolites of JWH-018 not being deactivated. Upon examination of the six recognized JWH-018 metabolites, the authors found that one of the metabolites had the same binding affinity as JWH-018, which is almost 10-fold higher than $\mathrm{THC}$, while four others had binding affinities nearly equivalent to that of $\mathrm{THC}^{3}$. A second paper 
studied the binding of CP47,497-C8 and JWH-073. The authors found that both compounds had comparable affinity and activity at $\mathrm{CB}_{1}$ as the previous mentioned study found with JWH- $018^{14}$. Later in 2013, a study compared the relative activities of a few synthetic cannabinoids and found that they declined in the order of JWH-122 > JWH-018 > JWH-210 > JWH-073 > THC ${ }^{43}$. Even more important for the current work is the 2017 finding of the UR-144 degradant maintaining a 4-fold higher binding affinity at $\mathrm{CB}_{1}$ that even that of its parent compound ${ }^{65}$. This was supported by Thomas et al. who investigated JWH-018, XLR-11 and PB-22's activity at both the $\mathrm{CB}_{1}$ and $\mathrm{CB}_{2}$ receptors ${ }^{66}$.

Poklis et al. reported the first detection and disposition of JWH-018 and JWH-073 in mice after inhalation of a common herbal incense product, "Magic Gold," in 2011. Five mice were sacrificed after 20 minutes and another five after 20 hours with both blood and brain samples being collected for subsequent analysis. Using an LC-MS/MS assay, it was determined that blood concentrations were lower than in a similar experiment using THC, while an equivalent concentration was found in the brain ${ }^{45}$. Another LC-MS/MS study, investigated the detection and disposition of JWH-018 in mice after inhalation of a different herbal incense product, "Buzz." This study determined the concentration of JWH-018 in the blood, brain, and liver of six mice sacrificed 20 minutes after exposure ${ }^{67}$. A third study, which sought to describe the behavioral effects observed after inhalation of the herbal incense product, "Buzz", in mice, found that the effects from "Buzz" were similar to marijuana in terms of magnitude and time course; however, equal doses of both substances yielded considerably lower brain levels of JWH-018 than THC. The authors concluded that the increased potency of JWH-018 compared to THC, combined with the variable amount of drug in many products, undoubtedly contribute to its risks ${ }^{45}$. 
A few studies have been completed in vitro to determine metabolic products of JWH-018, JWH-073, CP47, 497 and a newer generation compound, XLR-11. Typically, liver microsomes are used as the main cell line in the studies ${ }^{9,68-69}$. The most directly applicable to the research proposed here identified 13 phase 1 metabolites from JWH- $018^{68}$. Another study indicated that some phase 1 metabolites could be conjugated ${ }^{70}$. This research however aims to move into the realm of pyrolysis. Several drugs have been evaluated via pyrolysis including several in our laboratory $^{7,42,71-87}$. In the 1970 s and 80s, the focus was on PCP, heroin and cocaine and later moved into methamphetamine and fentany ${ }^{75,86}$. Recently a few reports were found on the synthetic cannabinoid UR-144 and a designer stimulant, mephedrone. A recent publication reviews various classes of drugs of abuse and their pyrolytic products that have been identified throughout the years ${ }^{10}$. However, only four studies have been found dealing with the metabolism of a pyrolytic product. The first three utilized the compound, UR-144, and the results of the study demonstrated that the pyrolytic products have independent metabolic pathways and thus can have additional toxicological properties. The pyrolysis products and their metabolites are indicated by GC-MS, LC-MS/MS, NMR, and LC-QTOF-MS ${ }^{7,42,72}$. Another study examined XLR-11 and its thermal degradation product, where the results show that the metabolites of the thermal degradation product were more prevalent than that of the parent compound ${ }^{88}$. These findings support the hypothesis underlying this proposed work that the pyrolytic products play a significant role in the context of intoxication, acute and chronic toxic effects, and potentially lethal toxic responses.

Three papers in 2014 were published evaluating the toxicity of synthetic cannabinoids. The first investigated CP-47,497-C8, and the authors used buccal and liver cells to determine the concentration in which toxicity is observed $^{89}$. The paper indicated that the drug caused 
interference with protein synthesis leading to membrane damage as well as DNA damage at concentrations of $\geq 7.5 \mu \mathrm{M}$ and $\geq 10 \mu \mathrm{M}$, respectively ${ }^{89}$. The second paper reviewed the toxicity of K2 products emphasizing that synthetic cannabinoids have higher potency and efficacy compared to THC, the synthetic cannabinoids have synergistic effects and that the metabolites maintain high affinity and activity at the $\mathrm{CB}_{1}$ and $\mathrm{CB}_{2}$ receptors ${ }^{90}$. The final paper evaluated the toxicity in brain cells. The authors first found that cell death was observed in a concentration dependent manner and that the cell death was triggered by the caspase- 3 cascade. By the use of $\mathrm{CB}_{1}$ and $\mathrm{CB}_{2}$ inhibitors, they also determined that the apoptotic pathway was controlled by the binding and activity at the $\mathrm{CB}_{1}$ receptor. Overall, the peak toxicity began at 2 hours after dosage and followed a $\mathrm{CB}_{1}$ mediated caspase-3 apoptotic cascade leading to neuronal damage and cell death $^{91}$.

Only recently has research been able to dive into the pharmacology of synthetic cannabinoids due to the rapid generations of new compounds. In 2016, an investigation focused on the tert-leucinate compounds such as 5F-AMB, and the authors utilized in vivo studies to demonstrate higher potency than that of earlier generation compounds. Additionally, they found that hypothermia was reversed by a $\mathrm{CB}_{1}$ antagonist, but not with $\mathrm{CB}_{2}{ }^{92}$. In 2017 , a review was conducted on the neurological effects of synthetic cannabinoids, and supported the notion that such drugs produce dependence ${ }^{93}$.

\subsubsection{Goals and Research Design}

The aim of this project was to examine the process of thermal degradation via the smoking process to understand the scope at which the products may affect analyses. The issues of synthetic cannabinoid toxicity acting as the major influence on the research and fully understanding the thermal degradation process are crucial due to the main mode of ingestion, 
smoking. There are current gaps in our understanding of cannabinoid effects including the mechanism of toxicity, and hypotheses span from unknown pharmacological activity to the observation of symptom overlap with serotonin syndrome. This research was conducted to determine the products, evaluate their presence in toxicological specimen and assess their possible connection to the additional toxicity of cannabinoid use observed.

The research design had three major phases; 1) Develop a pyrolysis apparatus and methodology 2) Determine the production of various thermal degradation products 3) Evaluate the presence of degradants in toxicological specimen. An apparatus with the capability to produce and collect the pyrolytic products across the volatility range within the means of the laboratory was the first step, and is outlined in Appendix B. After the apparatus was optimized, the second phase was to pyrolyze various synthetic cannabinoids and identify the thermal degradation products. This phase of the project is described in chapter 2 , and indicated a few common breakdown pathways to be used as a predictive model. The final phase was to evaluate the degradants presence in a toxicological specimen in order to establish that such products are not just produced, but also ingested. Chapter 4 outlines this investigation, and the detection of pyrolytics in blood samples is a strong indicator that such products cannot be dismissed in synthetic cannabinoid assessments.

\subsection{Instrumentation}

\subsubsection{Gas Chromatography - Mass Spectrometry (GC/MS)}

Gas chromatography - mass spectrometry has been a staple in the field of forensic science for years. A GC/MS instrument is utilized as a confirmatory analysis by GC separation of sample analytes and MS interpretation for analyte identification. The components of a GC/MS 
are displayed in Figure 1.1, including a carrier gas, sample injector, column contained in an oven, ionization source, mass analyzer, an electron multiplier and a data analysis system.

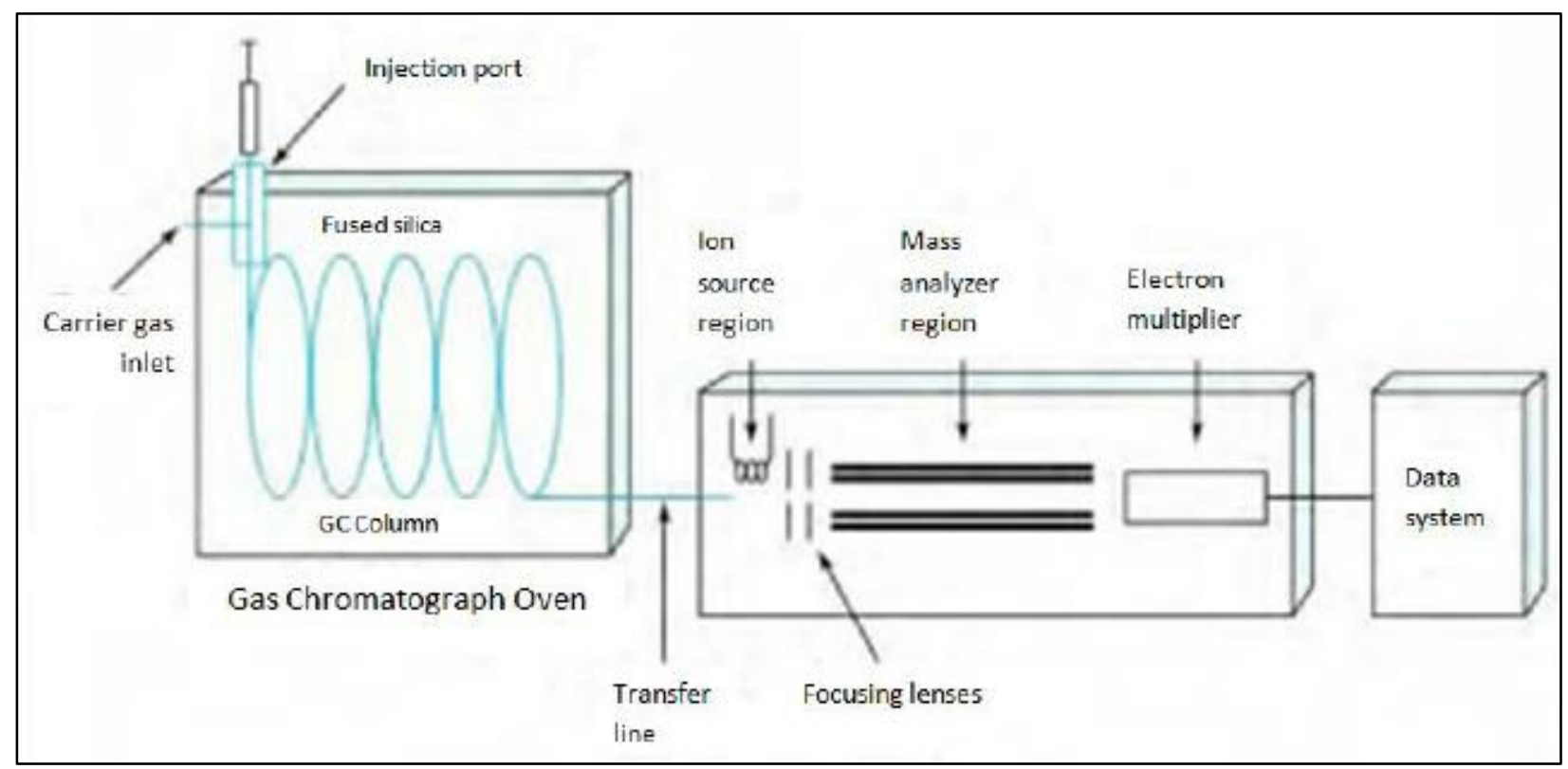

Figure 1.1 - Schematic of a gas chromatograph - mass spectrometer

The carrier gas and sample are injected into the column, which is held within a oven with controlled heat. As the heat is applied, the analytes within the sample are volatilized based on their individual properties, and separation is achieved as the analytes are carried through the column based on the partitioning of interaction between the mobile and stationary phases. The stationary phase is commonly a polysiloxane derivative that coats the inner walls of the silica glass capillary column, and the thickness can range from 0.5 to $5 \mu \mathrm{m}$. The oven that houses the capillary column is temperature controlled, which allows for temperature programming to facilitate separation. At lower temperatures, highly volatile analytes continue to flow, and as the temperature is ramped, the lower volatile analytes will begin to flow with the carrier gas.

As analytes elute off of the GC column, they are directed into the mass spectrometer. Ionization of the analytes occurs upon introduction, and this is commonly achieved by electron 
impact ionization (EI). In EI, the gaseous analytes are ionized via electron bombardment, typically at $70 \mathrm{eV}$, and the high-energy electrons cause the analyte to lose electrons and fragment due to the relaxation of the analyte from excited vibrational and rotational states. At this stage, there are charged fragments of various $\mathrm{m} / \mathrm{z}$, which are focused into the mass analyzer.

There are different types of mass analyzers, but the most common and type used in the studies of chapters $2 \& 3$ is a quadrupole. A quadrupole is comprised of four cylindrical rods arranged in parallel fashion with dc and ac (RF) voltages applied across each. The ions travel through the space between the four rods, and the voltages on the rods are simultaneously increased and alternated. This produces an oscillating trajectory onto the ions, which allows for separation of the ions based on their $\mathrm{m} / \mathrm{z}$ ratio. Upon exiting the quadrupole, the ions enter a detector, typically an electron multiplier, which amplifies and converts the ions into an electronic signal. The entirety of the mass spectrometer components is held under vacuum to ensure a free path of the ions throughout. The GC/MS analysis provides a chromatogram of retention time vs signal intensity, and a mass spectrum of $\mathrm{m} / \mathrm{z}$ versus abundance.

\subsubsection{Liquid Chromatography - Tandem Mass Spectrometry (LC/MS/MS)}

Liquid chromatography has become the standard technology in the field of forensic toxicology. LC/MS/MS analysis is a powerful confirmatory methodology, and a generic schematic is shown below in Figure 1.2. The mobile phase is comprised of different solvents that vary from aqueous based to more organic. A gradient mixture of the two solvents is used to elute analytes that are more soluble in either the aqueous or organic based solvent. Commonly, the initial mixture if highly aqueous, and throughout the gradient, the organic portion is increased over time before returning to the initial conditions. 


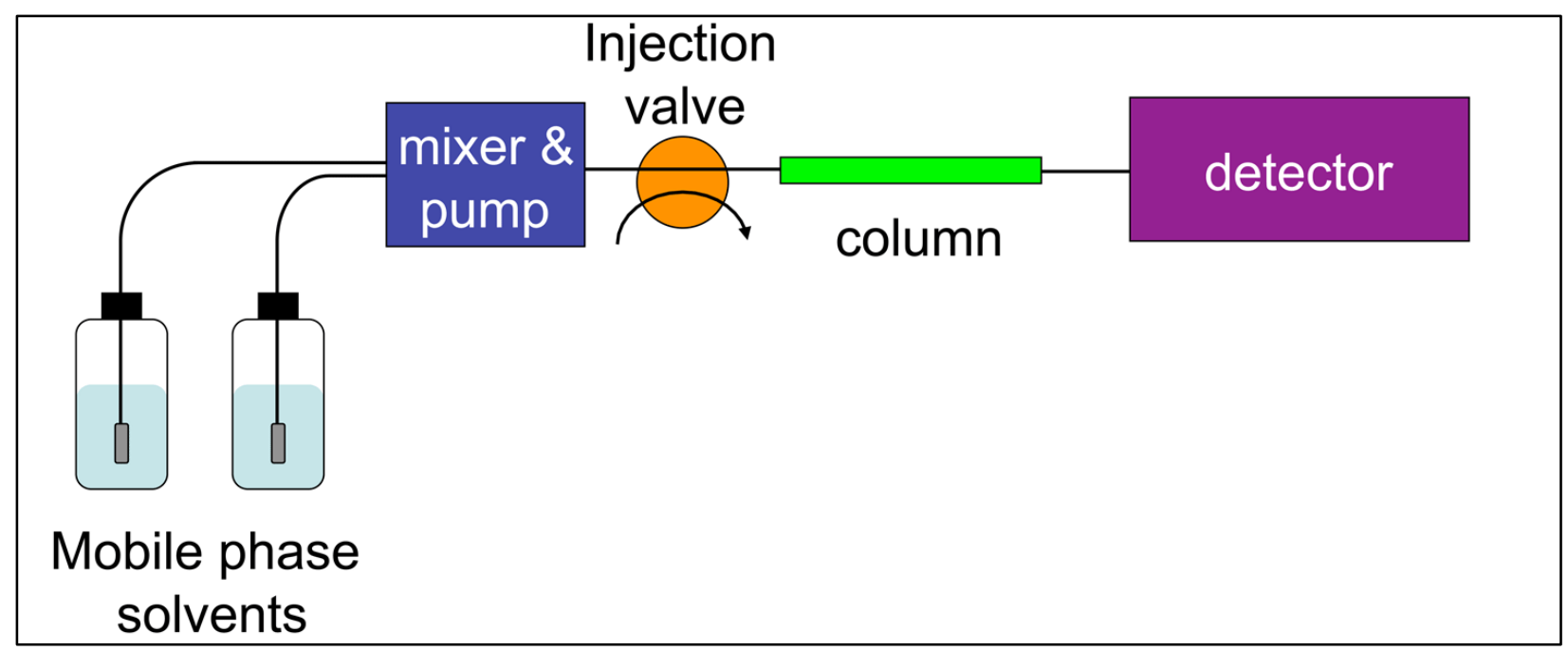

Figure 1.2 - Schematic of a liquid chromatograph

As the solvent are pumped towards the column, the injection valve is important in controlling sample volume before injection onto the column. Figure $\mathbf{1 . 3}$ outlines a common valve and the two settings used to control the flow of solvent/sample to waste or to the column. As shown by the different numbered circles, there are 6 different ports, and the inner connecting channels can rotate to connect different ports as seen in contrast between A and B. Initially, the channels are connected as in A. In this position, the mobile phase in channeled to the column alone with no sample. At this time, the sample is being flushed into the sample loop and eventually to waste. This is where the sample volume is controlled, as based on the sample flow, the desired sample volume will be pumped into the sample loop. Next, the channels switch to position B. Now, the mobile phase is pumped into the sample loop, which in turns forces the previously place desired sample volume from the sample loop to be introduced onto the column. The analytes within the sample are then separated based on their tendency to interact with the column and mobile phase gradient. 


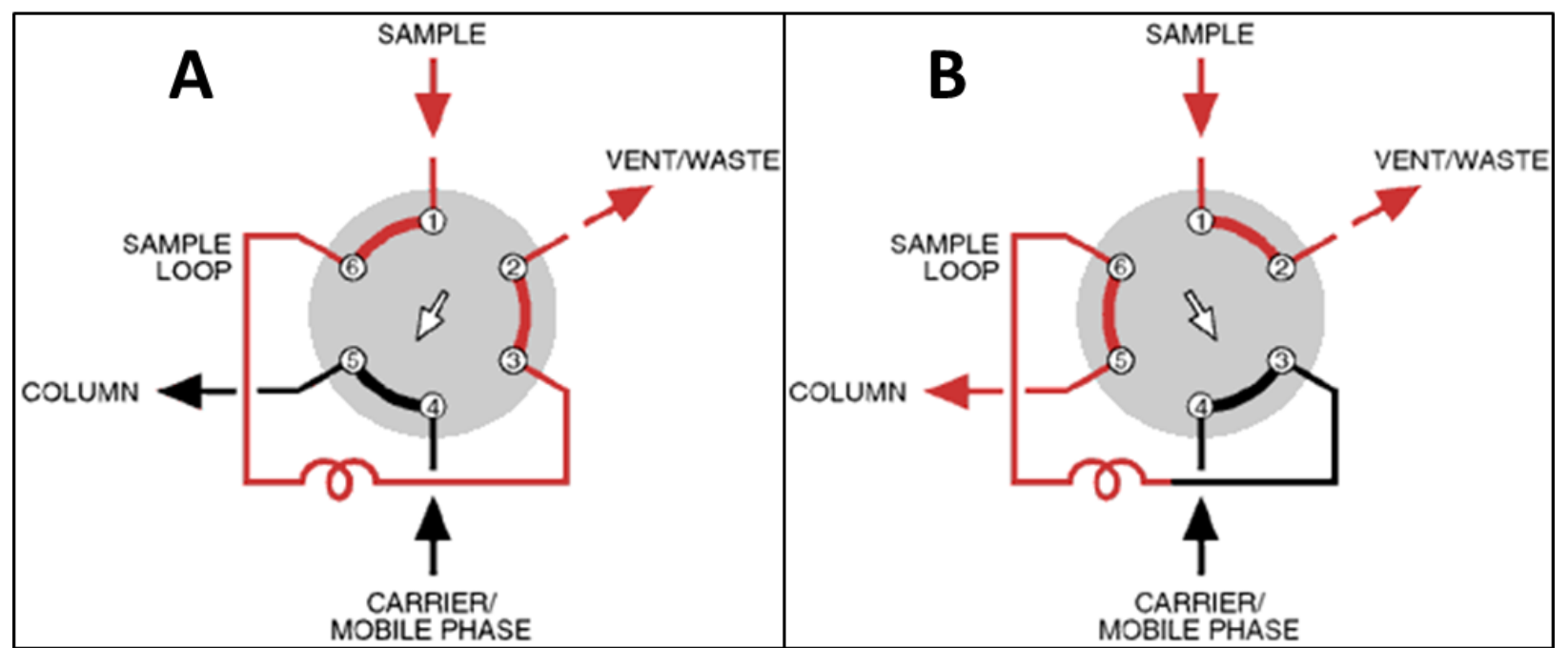

Figure 1.3 - Schematic of an LC injection valve. A: Flow setting to fill the sample loop with desired volume. B: Flow setting to flush sample volume out of the loop and onto column.

LC columns are manufactured in multiple ways to advantageously separate different types of analytes by placing different propertied chemical groups on the the silica packing. The most common type of column is a $\mathrm{C}_{18}$ column, which is a chain of 18 carbons producing a nonpolar stationary phase. Depending on the desired separation, columns with other groups such as phenyls can be can be utilized. In the studies within chapter 4 , a common $\mathrm{C}_{18}$ column was used for a reverse phase based separation. The column also vary in length and packing, which produces smaller pore sizes for mobile phase flow or a longer separation time if necessary. The specifics of the column used in the current study are outlined in chapter 4.

After separation is successful by the column, the analytes are introduced into the mass spectrometer, in which this case was a triple quadrupole tandem mass analyzer. Tandem mass spectrometers are classified into two categories; tandem-in-space or tandem-in-time. The instrument utilized in the current research is the most common, tandem-in-space instrument. The basic components of a triple quadrupole mass spectrometer instrument, depicted in Figure 1.4, are an ion source, three quadrupoles, and a detector. 


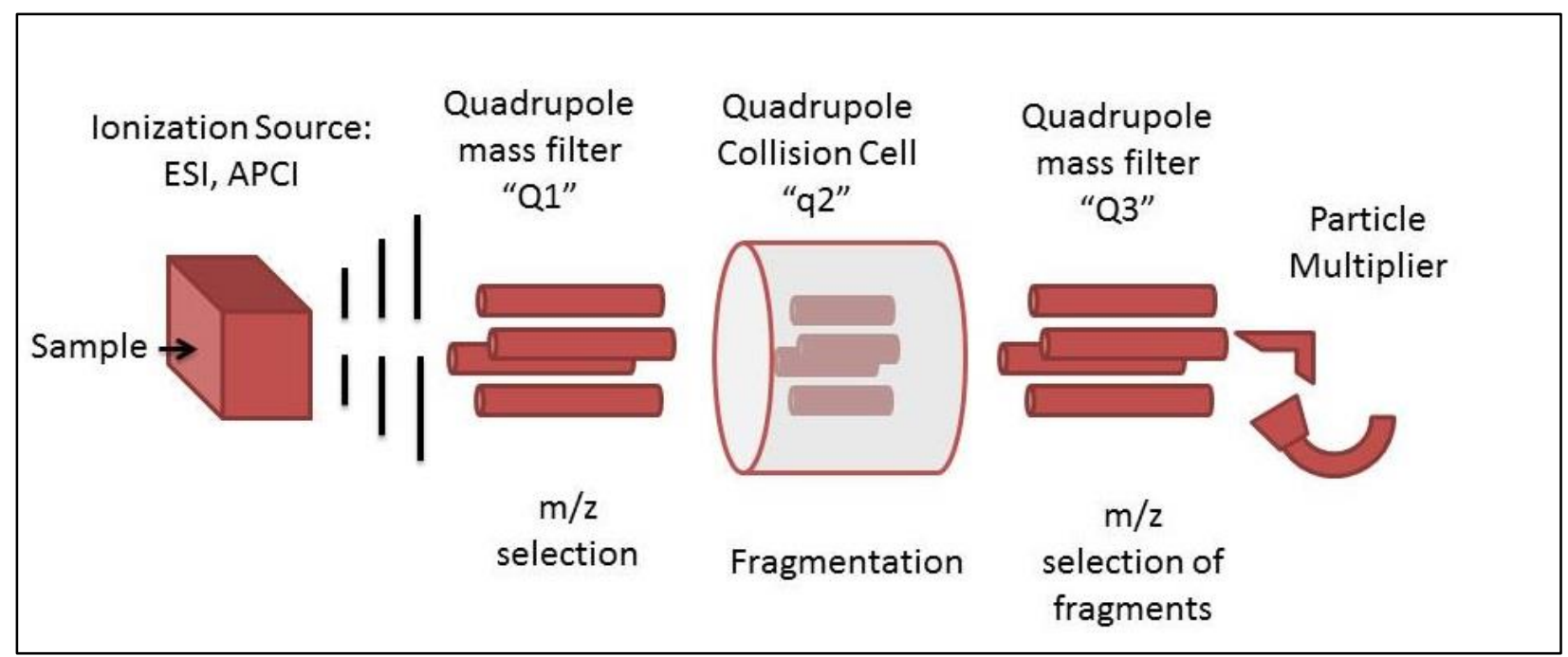

Figure 1.4 - Schematic of a triple quadrupole mass spectrometer

The ion source in this instrument employs electrospray ionization, ESI, source, which is a soft atmospheric pressure ionization technique in which ions transfer from solution into the gas phase. This technique uses differential pumping from atmospheric pressure to high vacuum, which is desired to allow for free movement of the analytes through the quadrupoles.

Solution at a flow rate of $1-20 \mu \mathrm{L} \mathrm{min}{ }^{-1}$ enters into a stainless-steel capillary held at a high potential of 3-4 kV. Upon exiting the capillary, the emerging liquid is under the influence of an electric field, causing charge separation. At the voltage in which pressure overcomes surface tension, a Taylor cone is formed and charged liquid emerges as droplets flowing in the direction of the counter electrode. A heated transfer capillary or curtain gas drives solvent evaporation. Solvent continuously evaporates and the electric field of the droplet increases as a result of the decreasing radius causing the droplets to undergo Coulomb fission repeatedly until ion formation. There are two models used to describe the formation of ions from charged droplets; charged-residue model (CRM) and ion evaporation model (IEM). The CRM describes the formation of ions through solvent evaporation and declustering. Conversely, the IEM model, describes the formation of ions through desorption from the droplet surface. 
Once formed, the ions flow through the orifice of the curtain plate into the first quadrupole, where the first stage of separation occurs. A quadrupole separates masses in the fashion described previously in Section 1.3.1. It is here, in the quadrupole, that precursor ions are selected. The precursor ions then enter the second quadrupole, which is used as the collision cell, where ions interact with collision gas to produce product ions. This process, called collision induced dissociation, CID, occurs and the resulting product ions pass into the third and final quadrupole for the second stage of mass separation. The ions are then detected and a tandem mass spectrum is produced. The ions are lastly detected and produce a tandem mass spectrum. Multiple reaction monitoring, MRM, was utilized to scan for multiple sets of transition ions for each analyte of interest. In using this method, it can further confirm the identification of an analyte by the observance of each transition and in the proper ion ratio.

\subsection{References}

1. Sobolevsky, T.; Prasolov, I.; Rodchenkov, G., Detection of JWH-018 metabolites in smoking mixture post-administration urine. Forensic science international 2010, 200 (1-3), 1417.

2. Wintermeyer, A.; Moller, I.; Thevis, M.; Jubner, M.; Beike, J.; Rothschild, M. A.; Bender, K., In vitro phase I metabolism of the synthetic cannabimimetic JWH-018. Analytical and bioanalytical chemistry 2010, 398 (5), 2141-53.

3. Brents, L. K.; Reichard, E. E.; Zimmerman, S. M.; Moran, J. H.; Fantegrossi, W. E.; Prather, P. L., Phase I hydroxylated metabolites of the K2 synthetic cannabinoid JWH-018 retain in vitro and in vivo cannabinoid 1 receptor affinity and activity. PloS one 2011, 6 (7), e21917.

4. Grigoryev, A.; Melnik, A.; Savchuk, S.; Simonov, A.; Rozhanets, V., Gas and liquid chromatography-mass spectrometry studies on the metabolism of the synthetic phenylacetylindole cannabimimetic JWH-250, the psychoactive component of smoking mixtures. Journal of chromatography. B, Analytical technologies in the biomedical and life sciences 2011, 879 (25), 2519-26.

5. Grigoryev, A.; Savchuk, S.; Melnik, A.; Moskaleva, N.; Dzhurko, J.; Ershov, M.; Nosyrev, A.; Vedenin, A.; Izotov, B.; Zabirova, I.; Rozhanets, V., Chromatography-mass spectrometry studies on the metabolism of synthetic cannabinoids JWH-018 and JWH-073, psychoactive components of smoking mixtures. Journal of chromatography. B, Analytical technologies in the biomedical and life sciences 2011, 879 (15-16), 1126-36.

6. Seely, K. A.; Brents, L. K.; Radominska-Pandya, A.; Endres, G. W.; Keyes, G. S.; Moran, J. H.; Prather, P. L., A major glucuronidated metabolite of JWH-018 is a neutral antagonist at CB1 receptors. Chemical research in toxicology 2012, 25 (4), 825-7. 
7. Grigoryev, A.; Kavanagh, P.; Melnik, A.; Savchuk, S.; Simonov, A., Gas and liquid chromatography-mass spectrometry detection of the urinary metabolites of UR-144 and its major pyrolysis product. Journal of analytical toxicology 2013, 37 (5), 265-76.

8. Rajasekaran, M.; Brents, L. K.; Franks, L. N.; Moran, J. H.; Prather, P. L., Human metabolites of synthetic cannabinoids JWH-018 and JWH-073 bind with high affinity and act as potent agonists at cannabinoid type- 2 receptors. Toxicology and applied pharmacology 2013, 269 (2), 100-8.

9. Wohlfarth, A.; Pang, S.; Zhu, M.; Gandhi, A. S.; Scheidweiler, K. B.; Liu, H. F.; Huestis, M. A., First metabolic profile of XLR-11, a novel synthetic cannabinoid, obtained by using human hepatocytes and high-resolution mass spectrometry. Clinical chemistry 2013, 59 (11), $1638-48$.

10. Bell, S.; Nida, C., Pyrolysis of drugs of abuse: a comprehensive review. Drug testing and analysis 2015, 7 (6), 445-56.

11. Bellia, F.; La Mendola, D.; Pedone, C.; Rizzarelli, E.; Saviano, M.; Vecchio, G., Selectively functionalized cyclodextrins and their metal complexes. Chemical Society Reviews 2009, 38 (9), 2756-2781.

12. Atwood, B. K.; Huffman, J.; Straiker, A.; Mackie, K., JWH018, a common constituent of 'Spice' herbal blends, is a potent and efficacious cannabinoid CB receptor agonist. British journal of pharmacology 2010, 160 (3), 585-93.

13. Vardakou, I.; Pistos, C.; Spiliopoulou, C., Spice drugs as a new trend: mode of action, identification and legislation. Toxicol Lett 2010, 197 (3), 157-62.

14. Atwood, B. K.; Lee, D.; Straiker, A.; Widlanski, T. S.; Mackie, K., CP47,497-C8 and JWH073, commonly found in 'Spice' herbal blends, are potent and efficacious CB(1) cannabinoid receptor agonists. European journal of pharmacology 2011, 659 (2-3), 139-45. 15. Rosenbaum, C. D.; Carreiro, S. P.; Babu, K. M., Here today, gone tomorrow...and back again? A review of herbal marijuana alternatives (K2, Spice), synthetic cathinones (bath salts), kratom, Salvia divinorum, methoxetamine, and piperazines. Journal of medical toxicology : official journal of the American College of Medical Toxicology 2012, 8 (1), 15-32.

16. Wiley, J. L.; Marusich, J. A.; Martin, B. R.; Huffman, J. W., 1-Pentyl-3phenylacetylindoles and JWH-018 share in vivo cannabinoid profiles in mice. Drug Alcohol Depend 2012, 123 (1-3), 148-53.

17. Scientific Working Group for Forensic Toxicology (SWGTOX) - Recommendations of the Research, Development, Testing, and Evaluation Committee*. Journal of analytical toxicology 2013, 37 (3), 187-191.

18. EVERY- PALMER, S., Warning: legal synthetic cannabinoid- receptor agonists such as JWH- 018 may precipitate psychosis in vulnerable individuals. Addiction 2010, 105 (10), 18591860.

19. Castellanos, D.; Singh, S.; Thornton, G.; Avila, M.; Moreno, A., Synthetic cannabinoid use: a case series of adolescents. The Journal of adolescent health : official publication of the Society for Adolescent Medicine 2011, 49 (4), 347-9.

20. Leonhart, M. M., Schedules of Controlled Substances; Temporary Placement of Five Synthetic Cannabinoids into Schedule I. Federal Register: Drug Enforcement Administration 2011, 76, 11075-11078.

21. Leonhart, M. M., Schedules of Controlled Substances: Placement of Five Synthetic Cannabinoids into Schedule I. Federal Register: Drug Enforcement Administration 2012, 77, 12508-12514. 
22. Seely, K. A.; Lapoint, J.; Moran, J. H.; Fattore, L., Spice drugs are more than harmless herbal blends: a review of the pharmacology and toxicology of synthetic cannabinoids. Progress in neuro-psychopharmacology \& biological psychiatry 2012, 39 (2), 234-43.

23. Marshell, R.; Kearney-Ramos, T.; Brents, L. K.; Hyatt, W. S.; Tai, S.; Prather, P. L.; Fantegrossi, W. E., In vivo effects of synthetic cannabinoids JWH-018 and JWH-073 and phytocannabinoid Delta(9)-THC in mice: Inhalation versus intraperitoneal injection. Pharmacol. Biochem. Behav. 2014, 124, 40-47.

24. Agency, D. E., Controlled Substances by CSA Schedule. 2018.

25. Auwarter, V.; Dresen, S.; Weinmann, W.; Muller, M.; Putz, M.; Ferreiros, N., 'Spice' and other herbal blends: harmless incense or cannabinoid designer drugs? Journal of mass spectrometry : JMS 2009, 44 (5), 832-7.

26. Fattore, L.; Fratta, W., Beyond THC: The New Generation of Cannabinoid Designer Drugs. Frontiers in behavioral neuroscience 2011, 5, 60.

27. Deluca, P., Alert on New Recreational Drugs on the Web; Building Up a European-Wide Digital Web Scan-Monitoring System. Project, P. W. M., Ed. King's College: 2012.

28. Piggee, C., Investigating a not-so-natural high. Analytical chemistry 2009, 81 (9), 3205-7.

29. Centers, A. A. o. P. C., Synthetic Marijuana Data. 2014, 1-4.

30. Poklis, J. L.; Amira, D.; Wise, L. E.; Wiebelhaus, J. M.; Haggerty, B. J.; Poklis, A., Detection and disposition of JWH-018 and JWH-073 in mice after exposure to "Magic Gold" smoke. Forensic science international 2012, 220 (1-3), 91-6.

31. Hudson, S.; Ramsey, J., The emergence and analysis of synthetic cannabinoids. Drug testing and analysis 2011, 3 (7-8), 466-78.

32. Howlett, A.; Johnson, M. R.; Melvin, L.; Milne, G., Nonclassical cannabinoid analgetics inhibit adenylate cyclase: development of a cannabinoid receptor model. Molecular pharmacology 1988, 33 (3), 297-302.

33. Ginsburg, B. C.; Schulze, D. R.; Hruba, L.; McMahon, L. R., JWH-018 and JWH-073: Delta(9)-tetrahydrocannabinol-like discriminative stimulus effects in monkeys. The Journal of pharmacology and experimental therapeutics 2012, 340 (1), 37-45.

34. Wiley, J. L.; Compton, D. R.; Dai, D.; Lainton, J. A.; Phillips, M.; Huffman, J. W.; Martin, B. R., Structure-activity relationships of indole-and pyrrole-derived cannabinoids. Journal of Pharmacology and Experimental Therapeutics 1998, 285 (3), 995-1004.

35. Zimmermann, U. S.; Winkelmann, P. R.; Pilhatsch, M.; Nees, J. A.; Spanagel, R.; Schulz, K., Withdrawal phenomena and dependence syndrome after the consumption of "spice gold". Deutsches Arzteblatt international 2009, 106 (27), 464-7.

36. Castaneto, M. S.; Gorelick, D. A.; Desrosiers, N. A.; Hartman, R. L.; Pirard, S.; Huestis, M. A., Synthetic cannabinoids: epidemiology, pharmacodynamics, and clinical implications. Drug Alcohol Depend 2014, 144, 12-41.

37. Wohlfarth, A.; Weinmann, W., Bioanalysis of new designer drugs. Bioanalysis 2010, 2 (5), 965-979.

38. AIT Among the First to Offer Blood Test for Synthetic Marijuana Compounds JWH-018 and JWH-073 (K2, Spice). 2-17-2011, 2011.

39. Meyer, M. R.; Maurer, H. H., Current status of hyphenated mass spectrometry in studies of the metabolism of drugs of abuse, including doping agents. Analytical and bioanalytical chemistry 2012, 402 (1), 195-208. 
40. Arntson, A.; Ofsa, B.; Lancaster, D.; Simon, J. R.; McMullin, M.; Logan, B., Validation of a novel immunoassay for the detection of synthetic cannabinoids and metabolites in urine specimens. Journal of analytical toxicology 2013, 37 (5), 284-290.

41. Favretto, D.; Pascali, J. P.; Tagliaro, F., New challenges and innovation in forensic toxicology: focus on the "New Psychoactive Substances". Journal of Chromatography A 2013, $1287,84-95$.

42. Kavanagh, P.; Grigoryev, A.; Savchuk, S.; Mikhura, I.; Formanovsky, A., UR-144 in products sold via the Internet: identification of related compounds and characterization of pyrolysis products. Drug testing and analysis 2013, 5 (8), 683-92.

43. Koller, V. J.; Zlabinger, G. J.; Auwarter, V.; Fuchs, S.; Knasmueller, S., Toxicological profiles of selected synthetic cannabinoids showing high binding affinities to the cannabinoid receptor subtype $\mathrm{CB}(1)$. Archives of toxicology 2013, 87 (7), 1287-97.

44. Seely, K. A.; Patton, A. L.; Moran, C. L.; Womack, M. L.; Prather, P. L.; Fantegrossi, W. E.; Radominska-Pandya, A.; Endres, G. W.; Channell, K. B.; Smith, N. H., Forensic investigation of K2, Spice, and "bath salt" commercial preparations: a three-year study of new designer drug products containing synthetic cannabinoid, stimulant, and hallucinogenic compounds. Forensic science international 2013, 233 (1-3), 416-422.

45. Wiebelhaus, J. M.; Poklis, J. L.; Poklis, A.; Vann, R. E.; Lichtman, A. H.; Wise, L. E., Inhalation exposure to smoke from synthetic "marijuana" produces potent cannabimimetic effects in mice. Drug Alcohol Depend. 2012, 126 (3), 316-323.

46. Penn, H. J.; Langman, L. J.; Unold, D.; Shields, J.; Nichols, J. H., Detection of synthetic cannabinoids in herbal incense products. Clinical biochemistry 2011, 44 (13), 1163-5.

47. Daw, R. C.; Grabenauer, M.; Pande, P. G.; Cox, A. O.; Kovach, A. L.; Davis, K. H.; Wiley, J. L.; Stout, P. R.; Thomas, B. F., Pyrolysis studies of synthetic cannabinoids in herbal products. Drug Alcohol Depend. 2014, 140, e44.

48. Teske, J.; Weller, J. P.; Fieguth, A.; Rothamel, T.; Schulz, Y.; Troger, H. D., Sensitive and rapid quantification of the cannabinoid receptor agonist naphthalen-1-yl-(1-pentylindol-3yl)methanone (JWH-018) in human serum by liquid chromatography-tandem mass spectrometry. Journal of chromatography. B, Analytical technologies in the biomedical and life sciences 2010, 878 (27), 2659-63.

49. $\quad$ Lindigkeit, R.; Boehme, A.; Eiserloh, I.; Luebbecke, M.; Wiggermann, M.; Ernst, L.; Beuerle, T., Spice: a never ending story? Forensic science international 2009, 191 (1-3), 58-63.

50. Dresen, S.; Ferreiros, N.; Putz, M.; Westphal, F.; Zimmermann, R.; Auwarter, V., Monitoring of herbal mixtures potentially containing synthetic cannabinoids as psychoactive compounds. Journal of mass spectrometry : JMS 2010, 45 (10), 1186-94.

51. Moller, I.; Wintermeyer, A.; Bender, K.; Jubner, M.; Thomas, A.; Krug, O.; Schanzer, W.; Thevis, M., Screening for the synthetic cannabinoid JWH-018 and its major metabolites in human doping controls. Drug testing and analysis 2011, 3 (9), 609-20.

52. Agency, W. A.-D., 2012 Prohibited List. 2011.

53. Dresen, S.; Kneisel, S.; Weinmann, W.; Zimmermann, R.; Auwarter, V., Development and validation of a liquid chromatography-tandem mass spectrometry method for the quantitation of synthetic cannabinoids of the aminoalkylindole type and methanandamide in serum and its application to forensic samples. Journal of mass spectrometry : JMS 2011, 46 (2), $163-71$. 
54. Borg, D.; Tverdovsky, A.; Stripp, R., A Fast and Comprehensive Analysis of 32 Synthetic Cannabinoids Using Agilent Triple Quadrupole LC-MS-MS. Journal of analytical toxicology 2016.

55. Knittel, J. L.; Holler, J. M.; Chmiel, J. D.; Vorce, S. P.; Magluilo, J., Jr.; Levine, B.; Ramos, G.; Bosy, T. Z., Analysis of Parent Synthetic Cannabinoids in Blood and Urinary Metabolites by Liquid Chromatography Tandem Mass Spectrometry. Journal of analytical toxicology 2016, 40 (3), 173-86.

56. Shanks, K. G.; Clark, W.; Behonick, G., Death Associated With the Use of the Synthetic Cannabinoid ADB-FUBINACA. Journal of analytical toxicology 2016, 40 (3), 236-9.

57. Tiscione, N. B.; Miller, R.; Shan, X.; Sprague, J.; Yeatman, D. T., An Efficient, Robust Method for the Determination of Cannabinoids in Whole Blood by LC-MS-MS. Journal of analytical toxicology 2016.

58. Usui, K.; Fujita, Y.; Kamijo, Y.; Kokaji, T.; Funayama, M., Identification of 5-Fluoro ADB in Human Whole Blood in Four Death Cases. Journal of analytical toxicology 2017, 1-5.

59. Chimalakonda, K. C.; Moran, C. L.; Kennedy, P. D.; Endres, G. W.; Uzieblo, A.; Dobrowolski, P. J.; Fifer, E. K.; Lapoint, J.; Nelson, L. S.; Hoffman, R. S.; James, L. P.; Radominska-Pandya, A.; Moran, J. H., Solid-phase extraction and quantitative measurement of omega and omega-1 metabolites of JWH-018 and JWH-073 in human urine. Analytical chemistry 2011, 83 (16), 6381-8.

60. Moran, C. L.; Le, V. H.; Chimalakonda, K. C.; Smedley, A. L.; Lackey, F. D.; Owen, S. N.; Kennedy, P. D.; Endres, G. W.; Ciske, F. L.; Kramer, J. B.; Kornilov, A. M.; Bratton, L. D.; Dobrowolski, P. J.; Wessinger, W. D.; Fantegrossi, W. E.; Prather, P. L.; James, L. P.; Radominska-Pandya, A.; Moran, J. H., Quantitative measurement of JWH-018 and JWH-073 metabolites excreted in human urine. Analytical chemistry 2011, 83 (11), 4228-36.

61. Carlier, J.; Diao, X.; Scheidweiler, K. B.; Huestis, M. A., Distinguishing Intake of New Synthetic Cannabinoids ADB-PINACA and 5F-ADB-PINACA with Human Hepatocyte Metabolites and High-Resolution Mass Spectrometry. Clinical chemistry 2017.

62. Carlier, J.; Diao, X.; Sempio, C.; Huestis, M. A., Identification of New Synthetic Cannabinoid ADB-CHMINACA (MAB-CHMINACA) Metabolites in Human Hepatocytes. AAPS J 2017, 19 (2), 568-577.

63. Kavanagh, P.; Grigoryev, A.; Krupina, N., Detection of metabolites of two synthetic cannabimimetics, MDMB-FUBINACA and ADB-FUBINACA, in authentic human urine specimens by accurate mass LC-MS: a comparison of intersecting metabolic patterns. Forensic Toxicology 2017.

64. Kim, M.; Kim, D. H.; Lee, Y. S.; Jang, C.-G.; Yang, C. H.; Lee, S., Changes in dopamine, serotonin and their metabolites in brain microdialysates from rats following exposure to new psychoactive drugs. Forensic Toxicology 2016.

65. Kaizaki-Mitsumoto, A.; Hataoka, K.; Funada, M.; Odanaka, Y.; Kumamoto, H.; Numazawa, S., Pyrolysis of UR-144, a synthetic cannabinoid, augments an affinity to human CB1 receptor and cannabimimetic effects in mice. The Journal of toxicological sciences 2017, 42 (3), 335-341.

66. Thomas, B. F.; Lefever, T. W.; Cortes, R. A.; Grabenauer, M.; Kovach, A. L.; Cox, A. O.; Patel, P. R.; Pollard, G. T.; Marusich, J. A.; Kevin, R. C.; Gamage, T. F.; Wiley, J. L., Thermolytic Degradation of Synthetic Cannabinoids: Chemical Exposures and Pharmacological Consequences. The Journal of pharmacology and experimental therapeutics 2017, 361 (1), 162171. 
67. Poklis, J. L.; Amira, D.; Wise, L. E.; Wiebelhaus, J. M.; Haggerty, B. J.; Lichtman, A. H.; Poklis, A., Determination of naphthalen-1-yl-(1-pentylindol-3-yl)methanone (JWH-018) in mouse blood and tissue after inhalation exposure to 'buzz' smoke by HPLC/MS/MS. Biomedical chromatography : BMC 2012, 26 (11), 1393-8.

68. Jin, M. J.; Lee, J.; In, M. K.; Yoo, H. H., Characterization of in vitro metabolites of CP 47,497, a synthetic cannabinoid, in human liver microsomes by LC-MS/MS. Journal of forensic sciences 2013, 58 (1), 195-9.

69. Mueller, D. M.; Rentsch, K. M., Generation of metabolites by an automated online metabolism method using human liver microsomes with subsequent identification by LC-MS (n), and metabolism of 11 cathinones. Analytical and bioanalytical chemistry 2012, 402 (6), 21412151.

70. Chimalakonda, K. C.; Bratton, S. M.; Le, V.-H.; Yiew, K. H.; Dineva, A.; Moran, C. L.; James, L. P.; Moran, J. H.; Radominska-Pandya, A., Conjugation of synthetic cannabinoids JWH-018 and JWH-073, metabolites by human UDP-glucuronosyltransferases. Drug metabolism and disposition 2011, 39 (10), 1967-1976.

71. Kavanagh, P.; O'Brien, J.; Power, J. D.; Talbot, B.; McDermott, S. D., 'Smoking'mephedrone: The identification of the pyrolysis products of 4-methylmethcathinone hydrochloride. Drug testing and analysis 2013, 5 (5), 291-305.

72. Adamowicz, P.; Zuba, D.; Sekula, K., Analysis of UR-144 and its pyrolysis product in blood and their metabolites in urine. Forensic science international 2013, 233 (1-3), 320-7.

73. Oldfield, L. S. a. B., S.C., Analysis of Selected Drugs of Abuse and Their Pyrolytic Products by LC/MS/MS. In SOFT, San Francisco, CA, 2011.

74. Nishikawa, R. K. a. B., S.C., Pyrolysis of Fentanyl: Isolation of Pyromarkers of Smoking Abuse. In SOFT, San Francisco, CA, 2011.

75. Nishikawa, R. K.; Bell, S. C.; Kraner, J. C.; Callery, P. S., Potential biomarkers of smoked fentanyl utilizing pyrolysis gas chromatography-mass spectrometry. Journal of analytical toxicology 2009, 33 (8), 418-422.

76. Gostič, T.; Klemenc, S.; Stefane, B., A study of the thermal decomposition of adulterated cocaine samples under optimized aerobic pyrolytic conditions. Forensic science international 2009, 187 (1-3), 19-28.

77. Shakleya, D. M.; Plumley, A. E.; Kraner, J. C.; Bell, S. C.; Callery, P. S., Trace evidence of trans-phenylpropene as a marker of smoked methamphetamine. Journal of analytical toxicology 2008, 32 (8), 705-708.

78. Nishikawa, R. K. a. B., S.C., Potential Biomarkers of Fentanyl Utilizing GC/MS. In SOFT, Phoenix, AZ, 2008.

79. Gayton-Ely, M.; Shakleya, D. M.; Bell, S. C., Application of a pyroprobe to simulate smoking and metabolic degradation of abused drugs through analytical pyrolysis. Journal of forensic sciences 2007, 52 (2), 473-8.

80. Shakleya, D. M.; Tarr, S. G.; Kraner, J. C.; Clay, D. J.; Callery, P. S., Potential marker for smoked methamphetamine hydrochloride based on a gas chromatography-mass spectrometry quantification method for trans-phenylpropene. Journal of analytical toxicology 2005, 29 (6), 552-555.

81. Sato, M.; Hida, M.; Nagase, H., Analysis of pyrolysis products of methamphetamine. Journal of analytical toxicology 2004, 28 (8), 638-643.

82. Lewis, R. J.; Johnson, R.; Angier, M.; Ritter, R., Determination of cocaine, its metabolites, pyrolysis products, and ethanol adducts in postmortem fluids and tissues using 
Zymark ${ }^{\circledR}$ automated solid-phase extraction and gas chromatography-mass spectrometry. Journal of Chromatography B 2004, 806 (2), 141-150.

83. Brenneisen, R.; Hasler, F., GC/MS Determination of Pyrolysis Products from Diacetylmorphine and Adulterants of Street Heroin Samples. Journal of forensic sciences 2002, 47 (4), 15456J.

84. Sato, M.; Hida, M.; Nagase, H., Analysis of pyrolysis products of dimethylamphetamine. Journal of analytical toxicology 2001, 25 (5), 304-309.

85. Lee, M.-R.; Jeng, J.; Hsiang, W.-S.; Hwang, B.-H., Determination of pyrolysis products of smoked methamphetamine mixed with tobacco by tandem mass spectrometry. Journal of analytical toxicology 1999, 23 (1), 41-45.

86. Sekine, H.; Nakahara, Y., Abuse of smoking methamphetamine mixed with tobacco: II. The formation mechanism of pyrolysis products. Journal of Forensic Science 1990, 35 (3), 580590 .

87. Brine, C. E. C. a. D. R., Pyrolysis products of heroin. Journal of forensic sciences 1985, 30 (1), 251-261.

88. Kanamori, T.; Kanda, K.; Yamamuro, T.; Kuwayama, K.; Tsujikawa, K.; Iwata, Y. T.; Inoue, H., Detection of main metabolites of XLR-11 and its thermal degradation product in human hepatoma HepaRG cells and human urine. Drug testing and analysis 2015.

89. Koller, V. J.; Auwarter, V.; Grummt, T.; Moosmann, B.; Misik, M.; Knasmuller, S., Investigation of the in vitro toxicological properties of the synthetic cannabimimetic drug CP47,497-C8. Toxicology and applied pharmacology 2014, 277 (2), 164-171.

90. Fantegrossi, W. E.; Moran, J. H.; Radominska-Pandya, A.; Prather, P. L., Distinct pharmacology and metabolism of K2 synthetic cannabinoids compared to Delta(9)-THC: mechanism underlying greater toxicity? Life sciences 2014, 97 (1), 45-54.

91. Tomiyama, K.; Funada, M., Cytotoxicity of synthetic cannabinoids on primary neuronal cells of the forebrain: the involvement of cannabinoid CB1 receptors and apoptotic cell death. Toxicology and applied pharmacology 2014, 274 (1), 17-23.

92. Banister, S. D.; Longworth, M.; Kevin, R.; Sachdev, S.; Santiago, M.; Stuart, J.; Mack, J. B.; Glass, M.; McGregor, I. S.; Connor, M.; Kassiou, M., Pharmacology of Valinate and tertLeucinate Synthetic Cannabinoids 5F-AMBICA, 5F-AMB, 5F-ADB, AMB-FUBINACA, MDMB-FUBINACA, MDMB-CHMICA, and Their Analogues. ACS Chem Neurosci 2016, 7 (9), 1241-54.

93. Miliano, C.; Serpelloni, G.; Rimondo, C.; Mereu, M.; Marti, M.; De Luca, M. A., Neuropharmacology of New Psychoactive Substances (NPS): Focus on the Rewarding and Reinforcing Properties of Cannabimimetics and Amphetamine-Like Stimulants. Front Neurosci 2016, 10, 153. 


\section{Chapter 2: Qualitative Analysis and Detection of the Pyrolytic Products of JWH-018 and 11 Additional Synthetic Cannabinoids in the Presence of Common Herbal Smoking Substrates}

This section of the research project evaluated the thermal degradation of 12 synthetic cannabinoids. It is important to state that the majority of the identifications in the below manuscript are tentative identifications based on any combination of a NIST library search match with a score of $\geq 75$, a mass spectrum analysis or based off of similarity to another reference standard, which is further described in this preface. There were a number of the pyrolytic products that were able to be confirmed with a reference standard, however as most of these products have yet to be identified, there was a lack of reference standards available. Table 2.1 below indicates the method of identification for the 52 thermal degradation products observed, and more detailed explanation of this process follows.

Table 2.1 - Overview of the identification method for the thermal degradation products observed in the chapter 2 manuscript. If not indicated as confirmed, it is a tentative identification.

\begin{tabular}{|l|l|}
\hline \multicolumn{1}{|c|}{ Pyrolytic Product } & \multicolumn{1}{c|}{ Method of Identification } \\
\hline 3-Hydroxyindazole & Based on Indazole NIST Hit \\
\hline N-(1,2-dimethylpropyl)-Cinnolinamine & Mass Spectrum Analysis \\
\hline 1-Methylcyclohexanylindazole & Mass Spectrum Analysis \\
\hline 1-methylcyclohexanyl-N-(valinamidyl)-Cinnolinamine & Mass Spectrum Analysis \\
\hline 1-Methylcyclohexanyl-3-Cinnolinamine & Mass Spectrum Analysis \\
\hline 1-Methylbenzylcinnoline & Mass Spectrum Analysis \\
\hline 1-Pentylindazole-3-carboxaldehyde & Mass Spectrum Analysis \\
\hline penten-4-yl-AB-PINACA & Based on AB-PINACA Standard \\
\hline 1-(5-fluoropentyl)-Indole & Mass Spectrum Analysis \\
\hline 1-(5-fluoropentyl)-Indole-3-carboxaldehyde & Mass Spectrum Analysis \\
\hline 3-Benzoylindole & Mass Spectrum Analysis \\
\hline 1-(5-fluoropentyl)-3-Benzoylindole & Mass Spectrum Analysis \\
\hline 3-Oxindole & Confirmed \\
\hline 1-Naphthalenecarbonitrile & Confirmed \\
\hline 2H-1-Benzopyran-2-one & Mass Spectrum Analysis \\
\hline Methoxynaphthalene or 2-methyl-Naphthol & Based on Naphthol Standard \\
\hline Naphthol & Confirmed \\
\hline 1H-Indole-3-ethanol & NIST Library Match \\
\hline
\end{tabular}




\begin{tabular}{|l|l|}
\hline penten-4-yl-JWH-018 & Based on JWH-018 Standard \\
\hline 1-butyl-Indole & Based on Indole Standard \\
\hline 1-butyl-Quinoline & Based on Quinoline Standard \\
\hline 1-Butylindole-3-carboxaldehyde & Mass Spectrum Analysis \\
\hline 1-butyl-3-Acetylindole & Mass Spectrum Analysis \\
\hline buten-3-yl-JWH-073 & Based on JWH-073 Standard \\
\hline penten-4-yl-JWH-081 & Based on JWH-081 Standard \\
\hline 1-Pentylquinoline & Based on Quinoline Standard \\
\hline N-penten-4-yl-JWH-210 & Based on JWH-210 Standard \\
\hline 3-(1-methylnaphthoyl)-Indole & Mass Spectrum Analysis \\
\hline N-propyl-3-(1-methylnaphthoyl)-Indole & Mass Spectrum Analysis \\
\hline UR-144 Degradant & Confirmed \\
\hline 1-(5-fluoropentyl)-Indole-3-carboxaldehyde & Mass Spectrum Analysis \\
\hline XLR-11 Degradant & Confirmed \\
\hline Naphthalene & Confirmed \\
\hline Quinoline & Confirmed \\
\hline Formylvaline amide & Mass Spectrum Analysis \\
\hline Indole & Confirmed \\
\hline Methylindole & Confirmed \\
\hline Hydroxyquinoline & Confirmed \\
\hline 4-Hydroxycinnoline & Confirmed \\
\hline 4-Cinnolinamine & NIST Library Match \\
\hline 1-Pentylindole & Mass Spectrum Analysis \\
\hline 1H-Indazole-3-carboxaldehyde & Confirmed \\
\hline 1-Pentylisatin & Based on Isatin Standard \\
\hline N-(2-methylpropyl)-4-Cinnolinamine & Mass Spectrum Analysis \\
\hline 1-Pentylindole-3-carboxaldehyde & Mass Spectrum Analysis \\
\hline 1-pentyl-3-Acetylindole & Mass Spectrum Analysis \\
\hline (1H-indol-3-yl)-(2,2,3,3-tetramethylcyclopropyl)-Methanone & Mass Spectrum Analysis \\
\hline 1-Methylindazole & Confirmed \\
\hline 1-Methylindazole-3-formamide & Mass Spectrum Analysis \\
\hline N-methyl-3-Naphthoylindole & Mass Spectrum Analysis \\
\hline N-phenyl-1-Naphthamide & NIST Library Match \\
\hline 3-Naphthoylindole & NIST Library Match \\
\hline JWH-018 & Confirmed \\
\hline
\end{tabular}

For the products in which there was no reference standard and no direct library match, a mass spectrum analysis was carried out based on any structural library hit, a plausible thermal breakdown from the parent compound and similarity to a reference standard. For example, a product such as 1-butyl-indole had no reference standard for comparison. Here the mass spectrum would be compared to that of the indole reference standard to establish a comparable 
fragmentation pattern other than additional fragments above the mass of indole and a parent peak of an extra $57 \mathrm{Da}$. Herein it is proposed that the additional $57 \mathrm{Da}$ is from a butyl chain bonded to the indole ring. This is supported by the fact that JWH-073 contains a butyl chain on the indole ring, which was the parent cannabinoid to produce this product. This remains a tentative identification, and is based on the structure of JWH-073 and observed MS of a reference standard, indole. This process was carried out on a number of the proposed pyrolytic products, but further confirmation is necessary. Another method for predicting the identification was to work off any library search hit. Here, if the library produced a high match probability for a compound such as indole, but the observed MS has a much higher mass molecular ion peak, it is clear that the product is not indole itself, though may contain an indole ring. In this case predictions were based on building possible structures from the parent cannabinoid and with the indole intact based on the library hit. This is by no means a confirmation, but a basis for the proposed pyrolytic product, in which further confirmation is necessary in supplemental research. This project has set the groundwork for identifying pyrolytic products, but is only the first step in this process. Next, it is needed to be reproduced, and reference standards synthesized to confirm any of the proposed products in the manuscript below. 


\section{Journal of \\ Analytical Toxicology}

Article Navigation

Qualitative Analysis and Detection of the Pyrolytic Products of JWH-O18 and 11 Additional Synthetic Cannabinoids in the Presence of Common Herbal Smoking Substrates

Stephen Raso $\mathbb{\Delta}$, Suzanne Bell

Journal of Analytical Toxicology, Volume 41, Issue 6, 1 July 2017, Pages 551-558, https://doi.org/10.1093/jat/bkx039 Published: 20 June 2017 Article history

Reproduced from the Journal of Analytical Toxicology. Qualitative Analysis and Detection of the Pyrolytic Products of JWH-O18 and 11 Additional Synthetic Cannabinoids in the Presence of Common Herbal Smoking Substrates. . S. Raso and S. Bell, Journal of Analytical Toxicology 41, 6, 551-558, 2017.

Permission was obtained from the co-authors and the Journal of Analytical Toxicology according to the journal's license's, copyright and permissions policy. A copy of the permissions agreement is shown in Appendix A.

Synthetic cannabinoids have become a ubiquitous challenge in forensic toxicology and seized drug analysis. Thermal degradation products have yet to be identified and evaluated for toxicity in comparison to parent and metabolic compounds. An investigation into these pyrolytic products, as the major route of ingestion is inhalation, may produce additional insight to understand the toxicity of synthetic cannabinoids. The pyrolysis of JWH-018 and 11 additional synthetic cannabinoids and six herbal plant substrates were conducted using an in-house constructed smoking simulator. After pyrolysis of herbal material alone, the plant substrate was spiked with the drug compounds to $2-5 \%$ w/w concentrations. Samples were collected, filtered, evaporated under nitrogen gas, reconstituted in methanol, and analyzed via gas chromatographmass spectrometer (GS/MS). Pyrolysis of the plant material alone produced 10 consistently observed compounds between the six plant species. The pyrolysis of the synthetic cannabinoids produced a total of 52 pyrolytic compounds, where 32 were unique to a particular parent compound and the remaining 20 were common products between multiple cannabinoids. The 
thermal degradation followed three major pathways that are outlined to assist in producing a predictive model for new synthetic cannabinoids that may arise in case samples. The observed pyrolytic products are also viable options for analysis in post mortem samples and the evaluation of toxicity.

\subsection{Introduction}

In recent years, notable progress has been made in identifying the evolving generations of synthetic cannabinoids and their corresponding metabolites. However, two areas remain relatively unexplored: 1) the toxicity and mechanism of toxicity of the parent drugs and their metabolites; and 2) identification of common pyrolytic products and their toxicity ${ }^{1-10}$. The latter is critical given that the most common mode of ingestion of synthetic cannabinoids is smoking or heated vapor inhalation. The acute toxicity of this abuse is of particular concern to forensic toxicology, medicolegal death investigation, and public health and safety.

Synthetic cannabinoid abuse appeared in the mid-2000s, primarily in Europe and soon thereafter in the United States ${ }^{11-15}$. These compounds mimic $\Delta^{9}$-tetrahydrocannabinol (THC), the active ingredient of cannabis; however, they produce acute toxic responses that are not observed with the use of $\mathrm{THC}^{11,14,16-19}$. The additional side effects, referred to as the "cannabinoid tetrad" include (1) hypothermia, (2) analgesia, (3) catalepsy and (4) suppression of locomotor activity ${ }^{20-}$ 26. Recent work in forensic toxicology in the context of synthetic cannabinoids has focused on analytical assays, and methods have been published for detecting synthetic cannabinoids along with their respective metabolites in toxicological matrices using hyphenated chromatographic/mass spectrometry instrumentation and immunoassays ${ }^{14,25,27-37}$. Lacking from the current literature are reports and data describing the synthetic cannabinoid pyrolytic products, mechanisms of toxicity of these species, and how this information could be used in the 
interpretation of forensic toxicology data. At least two authors have expressed the need to address these research gaps ${ }^{38-39}$. To date, few pyrolytic products have been identified with only two being observed in toxicological matrices, UR-144 and XLR-11 degradants ${ }^{28,40}$. Further identification of pyrolytic products may provide insight into the cause of additional acute toxicities.

The process of smoking is difficult to simulate given that the heated environment is thermally and chemically heterogeneous. Reaction zones of both oxidative and reductive chemistry may exist, altered by the process of inhalation. As a result, realistic and reproducible simulation of ingestion by smoking is difficult. Furthermore, there is no way to establish what compounds produced by smoking remain in inhaled air for delivery to the lungs and absorption into the bloodstream. In light of these considerations, exhaustive sampling and collection methods are a reasonable alternative as a starting point. A number of techniques have been used for conducting pyrolysis experiments, as summarized in a recent review, and include analytical pyroprobes, heating in sealed capillary tubes followed by extraction, and solvent trapping/impinge-like devices ${ }^{10}$. Each technique is geared toward collecting particular types of products. When utilizing a capillary tube or any apparatus to contain the sample while heating followed by extraction, the more volatile products are lost. Conversely, using an analytical pyroprobe, which connects to a GC inlet via a transfer line, may limit the sampling of the less volatile materials.

This paper describes results to date of a comprehensive characterization of the pyrolytic products associated with selected synthetic cannabinoids and six herbal substrates commonly seen in street samples. The work was accomplished using a device built in-house and designed to collect as many products as possible, from the most volatile to least. The analytical method was 
optimized using replicate and duplicate analyses starting with herbal mixtures without cannabinoids. These experiments established compounds that would be expected to arise from the substrate and to differentiate these from compounds arising from the synthetic cannabinoids. Using the optimized experimental procedures, each synthetic cannabinoids was characterized. The results were used to identify common pyrolytic pathways and to develop methods that will allow for prediction of pyrolytic products of new cannabinoids.

\subsection{Methods}

The experimental procedures spanned optimizing the constructed apparatus, evaluation of the designed method protocol, pyrolysis of herbal matrices and pyrolysis of synthetic cannabinoids. The apparatus was checked for air flow consistency and temperature to ensure adequate heat was acquired to induce pyrolysis. Initially, the method protocol specifically regarding sample collection was evaluated to optimize the collection of all pertinent produced products. Following the method checks, the herbal matrices were pyrolyzed to determine background pyrolytics, and finally the synthetic cannabinoid compounds were pyrolyzed.

\subsubsection{Materials and Reagents}

All portions of the apparatus involved directly in the pyrolysis are constructed of quartz or Teflon to endure high temperatures. Quartz glass tubing was obtained from Quartz Scientific, Incorporated (Fairport Harbor, $\mathrm{OH}$ ) in the following sizes: Inner diameter (I) of 1mm, outer diameter $(\mathrm{O})$ of $3 \mathrm{~mm}$ and wall width $(\mathrm{W})$ of $1 \mathrm{~mm} ; \mathrm{I}=7 \mathrm{~mm}, \mathrm{O}=9 \mathrm{~mm}$ and $\mathrm{W}=1 \mathrm{~mm}$; and $\mathrm{I}=10 \mathrm{~mm}$, $\mathrm{O}=12 \mathrm{~mm}$ and $\mathrm{W}=1 \mathrm{~mm}$. Quartz wool was purchased from Acros Organics (New Jersey, USA). Heat was applied using a Benzomatic ${ }^{\circledR}$ propane torch from Worthington Cylinder Corporation (Columbus, OH). Pressure levels were controlled with a model VP2S CPS Pro-Set Vacuum Pump (Hialeah, FL) and a pressure gauge from Valworx Incorporated (Cornelius, NC). The 
temperature was monitored using a HH506RA Multilogger Thermometer, a K-Type thermocouple and $0.20 \mathrm{~mm} \mathrm{CH}^{+}$and $0.20 \mathrm{~mm} \mathrm{Al}^{-}$wires from Omega Engineering, Incorporated (Stamford, CT). Sampling utilized 5mL LUER-SLIP plastic syringes and $17 \mathrm{~mm}, 0.45 \mu \mathrm{m}$ syringe filters from Thermo Fisher Scientific (Waltham, MA) with collection in Methanol from Fisher Scientific (Hampton, NH). Six herbal species including Nymphaea caerulea commonly called Blue Lotus, Nepeta cataria or Catnip, Turnera diffusa or Damiana, Zornia latolia or Maconha Brava, Althaea officinalis or Marshmallow Leaf and Combretum quadrangulare or Sakae Naa were purchased from Bouncing Bear Botanicals (Lawrence, KS). Synthetic cannabinoid standards of AB-CHMINACA, AB-FUBINACA, AB-PINACA, AM-694, AM-2201, JWH-018, JWH-073, JWH-081, JWH-210, MAM-2201, UR-144 and XLR-11 were purchased from Cayman Chemical Company (Ann Arbor, MI).

\subsubsection{Apparatus}

The in-house constructed apparatus utilized a T-junction of quartz tubing to contain the pyrolysis reaction, and positioned into a $250 \mathrm{~mL}$ Erlenmeyer vacuum flask via rubber stopper so that the tip of the tube sits in the capture solvent. The flask is connected to vacuum for consistent air flow producing a light bubbling in the capture solvent. Using the pressure gauge, a setting of 2 inHg was chosen, which equates to $6.77 \mathrm{kPa}$. A major issue with mimicking the smoking process is the inherent variability of the process, whether in a cigarette, "joint" or in any version of a bong. While it is difficult to evaluate how closely a given design mimics a smoking process, it is possible and essential to record at which temperature pyrolysis takes place. For temperature monitoring, thermocouple wires were placed in the reaction zone. The wires were kept apart with plastic coating and inserted through the shorter piece of the T-junction by a series of smaller quartz tubes held together with a Teflon plug to ensure to wires only came in contact, which 
enables temperature recording, within the reaction zone. A photograph of the constructed apparatus, schematic information of the T-junction and Teflon plug, and heat signature data is included in Appendix B.

\subsubsection{Sample Preparation}

The samples of herbal material needed to preparation, and were weighed out to constant masses and introduced to the apparatus. Each synthetic cannabinoid sample was prepared individually over a two to three-day period. The plant material mass was kept constant to ensure the pyrolysis atmosphere was consistent between trials, and the drug mass used was calculated thusly to produce a concentration level within the range of $2-5 \%$ by weight in milligrams. The synthetic cannabinoid standard was dissolved in methanol and sprayed onto the herbal material. This was conducted with movement of plant material in effort to homogenize spread of the drug compound. The sample sat overnight to allow the methanol to completely evaporate, leaving the drug compound behind on the herbal matrix. 


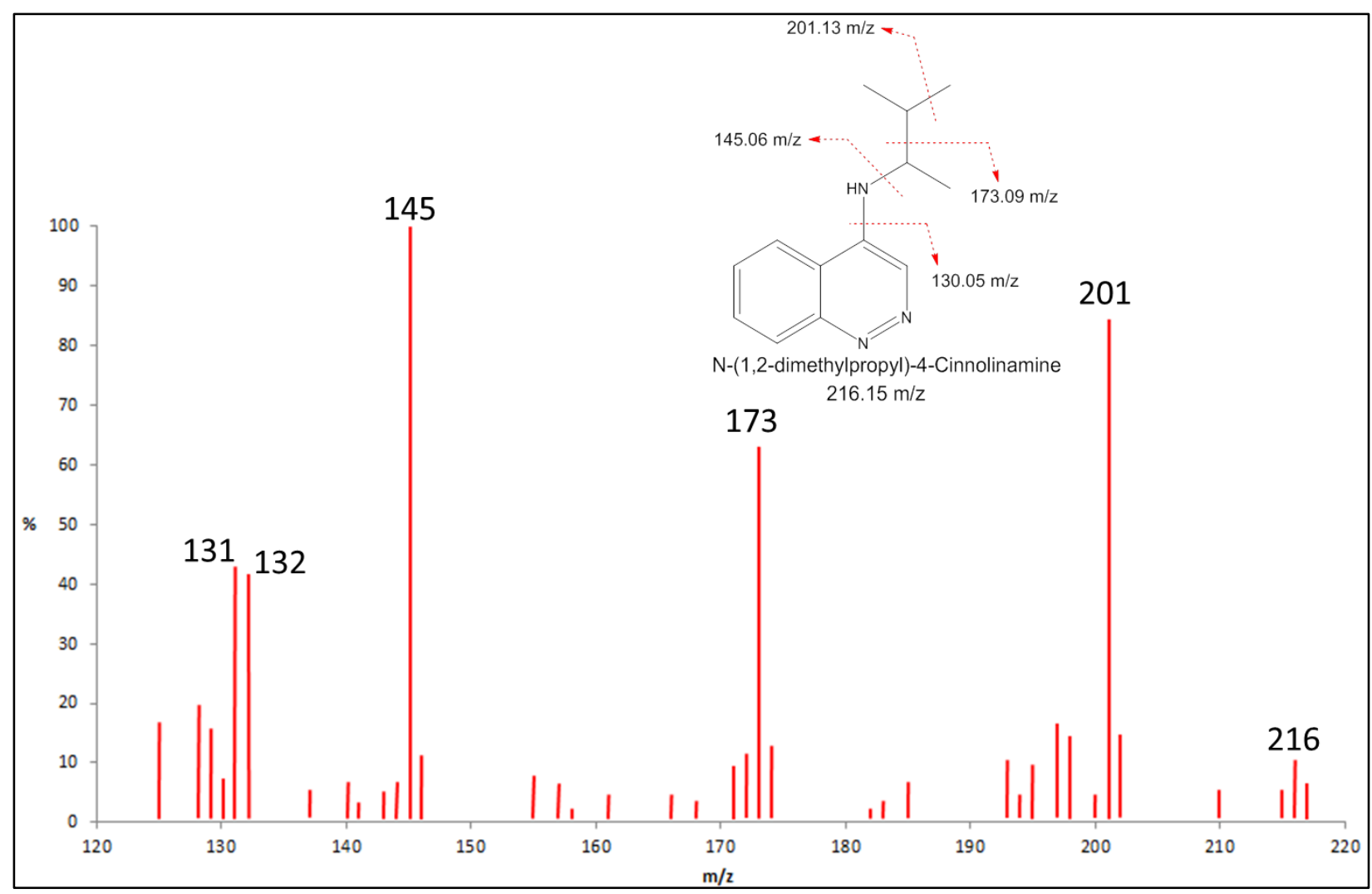

Figure 2.1 - A reproduced MS spectrum of predicted product, N-(1,2-dimethylpropyl)-4cinnolinamine, along with its predicted fragmentation pattern.

\subsubsection{Sample Collection}

Control samples from the apparatus and unburnt materials were initially analyzed. The collection method utilizes methanol as the solvent, so unburnt quartz wool and each herbal species in duplicate were soaked in methanol and analyzed via GC/MS. The quartz wool produced an equivalent spectrum to a methanol blank as expected. Any observed chromatographic peaks observed in both samples from the herbal material not seen in either the methanol or quartz blank were noted, and these background compounds were acknowledged as being present before herbal material and synthetic cannabinoid pyrolysis and thus not a pyrolytic product of interest. 
Table 2.2 - Proposed observed pyrolytic products unique to their respectively shown synthetic cannabinoid

\begin{tabular}{|c|c|}
\hline $\begin{array}{c}\text { Synthetic } \\
\text { Cannabinoid } \\
\end{array}$ & Pyrolytic Product \\
\hline \multirow{5}{*}{ AB-CHMINACA } & 3-Hydroxyindazole \\
\hline & N-(1,2-dimethylpropyl)-Cinnolinamine \\
\hline & 1-Methylcyclohexanylindazole \\
\hline & 1-methylcyclohexanyl-N-(valinamidyl)-Cinnolinamine \\
\hline & 1-Methylcyclohexanyl-3-Cinnolinamine \\
\hline AB-FUBINACA & 1-Methylbenzylcinnoline \\
\hline \multirow{2}{*}{ AB-PINACA } & 1-Pentylindazole-3-carboxaldehyde \\
\hline & penten-4-yl-AB-PINACA \\
\hline \multirow{4}{*}{ AM-694 } & 1-(5-fluoropentyl)-Indole \\
\hline & 1-(5-fluoropentyl)-Indole-3-carboxaldehyde \\
\hline & 3-Benzoylindole \\
\hline & 1-(5-fluoropentyl)-3-Benzoylindole \\
\hline AM-2201 & 3-Oxindole \\
\hline \multirow{6}{*}{ JWH-018 } & 1-Naphthalenecarbonitrile \\
\hline & 2H-1-Benzopyran-2-one \\
\hline & Methoxynaphthalene or 2-methyl-Naphthol \\
\hline & Naphthol \\
\hline & 1H-Indole-3-ethanol \\
\hline & penten-4-yl-JWH-018 \\
\hline \multirow{5}{*}{ JWH-073 } & 1-butyl-Indole \\
\hline & 1-butyl-Quinoline \\
\hline & 1-Butylindole-3-carboxaldehyde \\
\hline & 1-butyl-3-Acetylindole \\
\hline & buten-3-yl-JWH-073 \\
\hline JWH-081 & penten-4-yl-JWH-081 \\
\hline \multirow{2}{*}{ JWH-210 } & 1-Pentylquinoline \\
\hline & N-penten-4-yl-JWH-210 \\
\hline \multirow{2}{*}{ MAM-2201 } & 3-(1-methylnaphthoyl)-Indole \\
\hline & N-propyl-3-(1-methylnaphthoyl)-Indole \\
\hline UR-144 & UR-144 Degradant \\
\hline \multirow{2}{*}{ XLR-11 } & 1-(5-fluoropentyl)-Indole-3-carboxaldehyde \\
\hline & XLR-11 Degradant \\
\hline
\end{tabular}


Upon completion of a given burning experiment, three samples are collected from the apparatus. From the reaction zone, the quartz wool plugs and any leftover ashes are transferred to a 5mL LUER-SLIP plastic syringe fitted with a $0.45 \mu \mathrm{m}$ filter. The contents are filtered with $5 \mathrm{~mL}$ of methanol and collected in a $10 \mathrm{~mL}$ vial as the first sample. The second sample is also collected into a $10 \mathrm{~mL}$ vial after rinsing the T-junction tube with $10 \mathrm{~mL}$ of methanol. The capture solvent from the $250 \mathrm{~mL}$ vacuum flask, which is $50 \mathrm{~mL}$ of methanol, is transferred to a $100 \mathrm{~mL}$ beaker as the third sample. All samples were evaporated under nitrogen and reconstituted in methanol. The capture solvent was reconstituted to $10 \mathrm{~mL}$, transferred to a $10 \mathrm{~mL}$ vial, re-evaporated and reconstituted to a final volume of $2 \mathrm{~mL}$. The rinse from the tube was reconstituted to a final volume of $2 \mathrm{~mL}$, and the quartz/ashes sample was reconstituted to $500 \mu \mathrm{L}$.

\subsubsection{Instrumental Analysis}

Analyses were performed using a Perkin Elmer Clarus 500 gas chromatograph with a Restek Rxi ${ }^{\circledR}-5$ Sil with Integra-Guard ${ }^{\circledR} 30 \mathrm{~m}$ x $250 \mu \mathrm{m} \times 0.5 \mu \mathrm{m}$ column coupled with a Clarus SQ8T mass spectrometer. Helium was used as the carrier gas with a flow of $12.1 \mathrm{~mL} / \mathrm{min}$. The injection volume was a splitless $2 \mu \mathrm{L}$ with the heater set at $275^{\circ} \mathrm{C}$. The gas chromatography (GC) method had an initial temperature of $100^{\circ} \mathrm{C}$, ramp of $20^{\circ} \mathrm{C} / \mathrm{min}$ and was held at $320^{\circ} \mathrm{C}$ for $15 \mathrm{~min}$. The mass spectrometer (MS) was set with a 1.00min solvent delay, scan time of $0.2 \mathrm{sec}$ and a range of $40-500 \mathrm{~m} / \mathrm{z}$. 


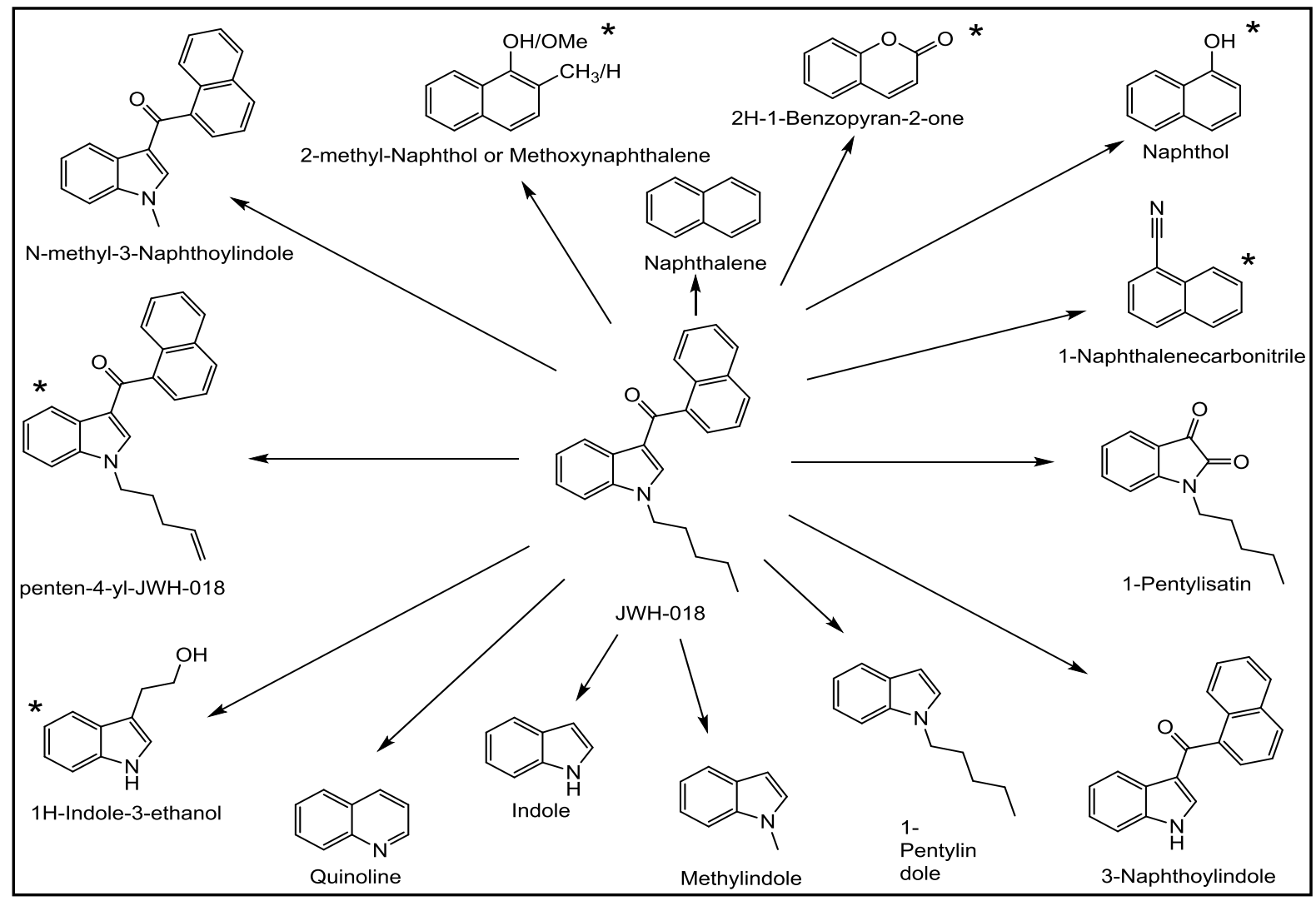

Figure 2.2 - Breakdown chart of JWH-018 to its proposed observed pyrolytic products; (*) unique products.

\subsubsection{Synthetic Cannabinoid Pyrolysis}

Prior to analyzing the synthetic cannabinoids, compounds that arose from pyrolysis of the herbal material alone were characterized. This process and results are described in Appendix B, and the results were consistent with previous plant pyrolysis studies demonstrating that the current methodology is suitable for the present application.

An optimization of experimental conditions was performed to determine the number of replicates and the number of extractions and rinses necessary to maximize recovery of pyrolytic products from the synthetic cannabinoids. This was accomplished through a series of 5 replicate experiments using JWH-018 on Damiana with exhaustive sampling. This sampling schematic is outlined in Appendix B. Results of these experiments demonstrated that a single wash is 
necessary for both quartz/ashes and tube samples. Additionally, consistent data was observed through the five trials, and it was determined that three trials was sufficient to acquire all variations of products. As a result, the remaining JWH-018 trials on the other five herbal matrices was carried out at an $n=3$ using only a single rinse for sample collection. Upon completion of JWH-018 analyses, no differentiation of results was observed between the six herbal matrices. The remaining 11 synthetic cannabinoid analyses were performed using the same parameters as JWH-018, but only on a single herbal matrix, Damiana.

\subsubsection{Data Analysis}

The data analysis stepwise limited the chromatographic peaks of interest to those consistently observed. Initially, chromatographic peaks were limited to those with a signal to noise ratio greater than 5 , as calculated by TurboMass 6.1 .0 software. They were further narrowed down those consistently present in at least two of the three trials within a retention time window of $+/-0.05 \mathrm{~min}$. The remaining peaks were noted as a possible pyrolytic product and evaluated for tentative identification. The identification process is difficult for such products due to the lack of standards available. Initially, a library search using the National Institute of Standards and Technology (NIST/NIH) database was carried out to obtain a starting point of a possible general structure. Mass spectrum analysis was conducted to insure the fragment breakdown was plausible for the predicted pyrolytic compound, and an example of this is shown in Figure 2.1. Additionally, the chromatography was evaluated via retention index (RI) where possible. This combined data analysis along with a logical mechanism of production via pyrolysis was used to predict compound identifications, but these were not confirmed with reference materials as they are largely unavailable. 


\subsection{Results}

\subsubsection{Synthetic Cannabinoid Pyrolysis}

The pyrolysis trials of the synthetic cannabinoids yielded 52 pyrolytic products, and each was found to have presence in the smoke as detected in either the tube or solvent samples, with little to none detected left behind in the quartz/ashes sample. This indicates a potential for harm as they travel within the inhaled smoke or off to the local atmosphere and may affect an innocent bystander. Of the proposed pyrolytic products, 32 were unique to a particular parent compound studied. The unique products might be exploitable as additional markers of synthetic cannabinoid use even when the parent compound is not detected. Assuming that these pyrolytic products reach the body and are absorbed, this may widen the scope of toxicological analysis by providing analytes outside of the typical parent and metabolic compounds. Further studies into the metabolism, pharmacokinetics and pharmacodynamics are necessary to understand the effects of these products after production via the smoking process. Table 2.2 lists the suggested products along with their respective parent synthetic cannabinoid, and Figure 2.2 outlines an example breakdown of parent to the predicted pyrolytic products. Two important pyrolytics to note were the degradants of UR-144 and XLR-11, as these are the two previously detected pyrolytics within toxicological samples, and their presence strengthens the utility of the current method as it is in agreement with expected pyrolytic compounds previously detected ${ }^{28,40}$. Additionally, Daw et al reported a possible pyrolytic trend of a 2 hydrogen loss at the end of the $\mathrm{N}$-alkyl chain where present ${ }^{39}$. This trend was observed here with synthetic cannabinoids AB-PINACA, and JWH-018, -073, -081 and -210.

Figure 2.2 further illustrates examples of three common breakdown tendencies across the pyrolysis of all 12 synthetic cannabinoids. The first is a break on either side of the central 
carbonyl group commonly present in synthetic cannabinoids. This breakdown trend produces pyrolytics such as indole or naphthalene products. The second trend, predictable due to the weak $\mathrm{C}-\mathrm{N}$ bond, is the loss of the substituent group bonded to the nitrogen of the indole or indazole ring structures. An example of this in Figure2.2 is the proposed product, 3-Naphthoylindole, or other such products such as Formylvaline amide from AB-PINACA and 3-Benzoylindole from AM-694. The last is the common pyrolytic process of a ring size increase. Majority of synthetic cannabinoids have either an indole or indazole ring structure, and with the pyrolytic ring size increase, they convert to a quinoline or cinnoline ring structure respectively. These breakdown or conversion trends can be used for a prediction model for the thermal degradation of the continually evolving generations of synthetic cannabinoids.

Separate from the unique pyrolytic products for each cannabinoid, the remaining 20 suggested products were shared between at least two of the parent synthetic cannabinoids studied, typically similar in structural class. These products are outlined in Table 2.3 and indicate which parent compounds produced each pyrolytic product. The detection of these products could not be used to indicate the use of any specific synthetic cannabinoid, but can be an indication for the use of synthetic cannabinoids in general or possibly limit the search to a structural class such as naphthoylindoles, indazoles or tetramethylcyclopropyls. Figure $\mathbf{2 . 3}$ shows an example breakdown of the different parent synthetic cannabinoids that produced the pyrolytic, quinoline. It can be noted that each parent compound includes an indole group, and this type of trend could be exploited to limit a search to those with an indole moiety if quinoline was detected during analysis if additional information points toward synthetic cannabinoid use. A limitation here would be other drug compounds that may also share quinoline as a pyrolytic product, but is still useful as a starting point during investigation. 
Table 2.3 - Proposed pyrolytic products with the parent synthetic cannabinoids they are commonly produced from; a) AB-CHMINACA b) AB-FUBINACA c) AB-PINACA d) AM-694 e) AM-2201 f) JWH-018 g) JWH-073 h) JWH-081 i) JWH-210 j) MAM-2201 k) UR-144 and l) XLR-11

\begin{tabular}{|l|c|}
\hline \multicolumn{1}{|c|}{ Pyrolytic Product } & Parent Compounds \\
\hline Naphthalene & $\mathrm{f}, \mathrm{g}, \mathrm{h}$ \\
\hline Quinoline & $\mathrm{e}, \mathrm{f}, \mathrm{g}, \mathrm{h}, \mathrm{i}, \mathrm{j}, \mathrm{k}$ \\
\hline Formylvaline amide & $\mathrm{a}, \mathrm{c}$ \\
\hline Indole & $\mathrm{e}, \mathrm{f}, \mathrm{g}, \mathrm{h}, \mathrm{i}, \mathrm{j}$ \\
\hline Methylindole & $\mathrm{e}, \mathrm{f}$ \\
\hline Hydroxyquinoline & $\mathrm{g}, \mathrm{h}, \mathrm{j}$ \\
\hline 4-Hydroxycinnoline & $\mathrm{b}, \mathrm{c}$ \\
\hline 4-Cinnolinamine & $\mathrm{f}, \mathrm{l}$ \\
\hline 1-Pentylindole & $\mathrm{b}, \mathrm{c}$ \\
\hline 1H-Indazole-3-carboxaldehyde & $\mathrm{f}, \mathrm{l}$ \\
\hline 1-Pentylisatin & $\mathrm{a}, \mathrm{b}, \mathrm{c}$ \\
\hline N-(2-methylpropyl)-4-Cinnolinamine & $\mathrm{d}, \mathrm{e}, \mathrm{h}, \mathrm{i}, \mathrm{j}, \mathrm{k}, \mathrm{l}$ \\
\hline 1-Pentylindole-3-carboxaldehyde & $\mathrm{e}, \mathrm{h}, \mathrm{i}, \mathrm{j}, \mathrm{k}, \mathrm{l}$ \\
\hline 1-pentyl-3-Acetylindole & $\mathrm{k}, \mathrm{l}$ \\
\hline (1H-indol-3-yl)-(2,2,3,3-tetramethylcyclopropyl)-Methanone & $\mathrm{a}, \mathrm{b}$ \\
\hline 1-Methylindazole & $\mathrm{b}, \mathrm{c}$ \\
\hline 1-Methylindazole-3-formamide & $\mathrm{f}, \mathrm{g}, \mathrm{i}$ \\
\hline N-methyl-3-Naphthoylindole & $\mathrm{g}, \mathrm{i}$ \\
\hline N-phenyl-1-Naphthamide & $\mathrm{f}, \mathrm{h}, \mathrm{j}$ \\
\hline 3-Naphthoylindole & $\mathrm{e}, \mathrm{h}$ \\
\hline JWH-018 & \\
\hline
\end{tabular}

\subsection{Discussion}

Due to the major route of ingestion of synthetic cannabinoids being inhalation through a smoking process, an understanding of the pyrolytic breakdown of these compounds is important for full analysis in toxicological samples. Pyrolysis has been studied in the past with different techniques; however they had the tendency to focus on either the most or least volatile products. There is lacking data on the majority of products' volatility, but a few where there is, indicate the ability of the reported methodology to collect products across the volatility range. A few high 
volatile product examples are indole, quinoline and naphthalene whose vapor pressure levels are on the order of $\sim 10^{-2} \mathrm{mmHg}^{41-43}$. On the other side of the spectrum, low volatile parent compounds such as JWH-018 and JWH-073 whose vapor pressure is on the order of $\sim 10^{-10}$ mmHg were also collected ${ }^{44-45}$.

The constructed apparatus demonstrated the ability to produce a "smoking-like environment", which is important as synthetic cannabinoids are often smoked using an herbal matrix laced with the compound of interest. Six common herbal materials were pyrolyzed to determine background products to differentiate from those pyrolytics of synthetic cannabinoids, and ten consistent products, which were tentatively identified, were detected and consistent with previous pyrolysis studies on plant material demonstrating the methodology was fit for pyrolytic analyses.

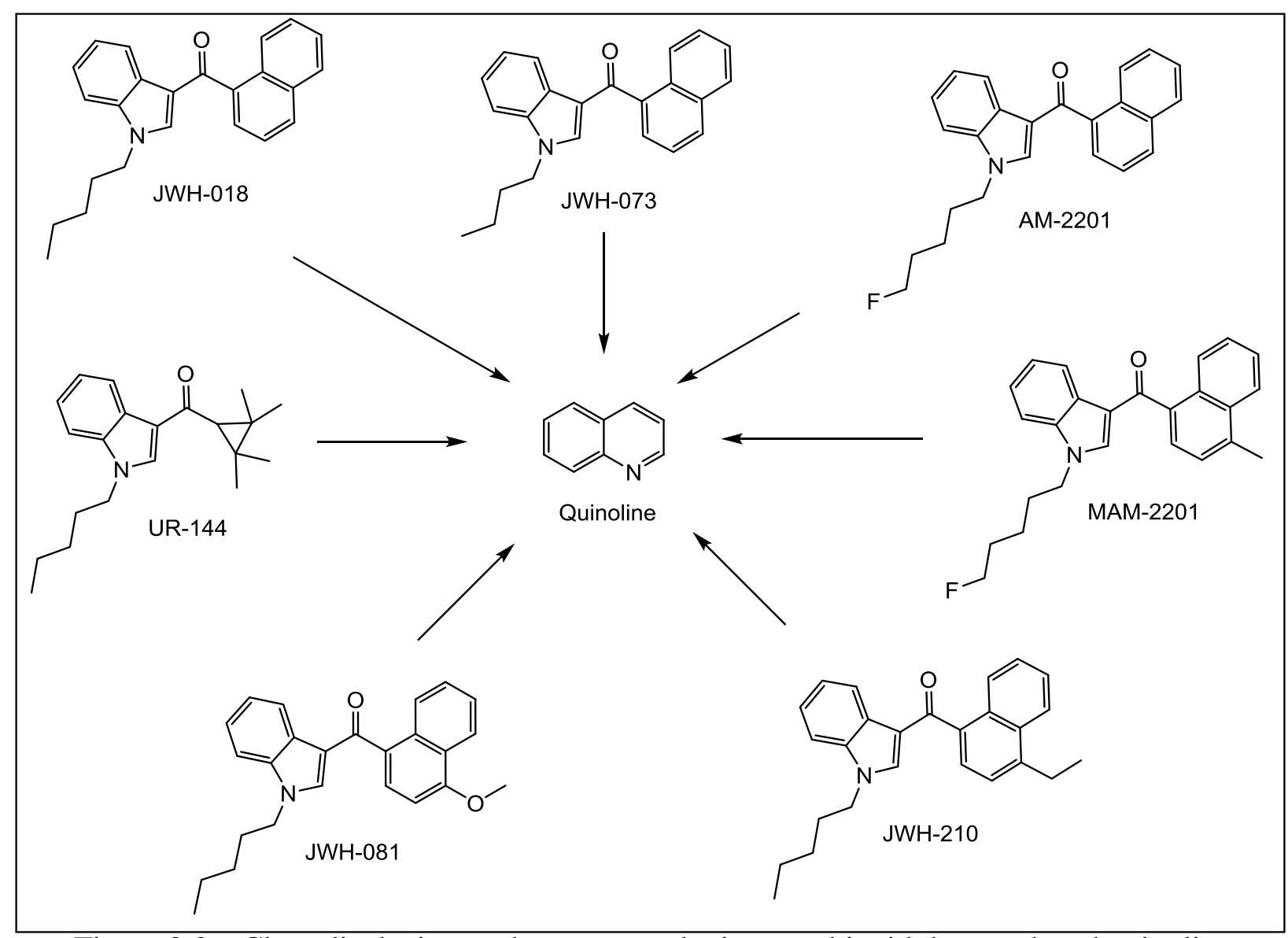

Figure 2.3 - Chart displaying each parent synthetic cannabinoid that produced quinoline. 
The pyrolysis trials of the synthetic cannabinoids produced fifty two pyrolytic products. Thirty two of these proposed products were unique to a particular parent cannabinoid compound, whereas the remaining twenty were shared by multiple parent compounds. The unique pyrolytic products are important, as they may serve as additional toxicological markers and indicate use of a specific synthetic cannabinoid without detection of the parent or metabolic compound. The shared pyrolytic products are not an indication of a specific cannabinoid, but are a useful suggestion of synthetic cannabinoid use. Another powerful tool for these thermal degradants is for the analysis of drug paraphernalia for the presence of synthetic cannabinoids. Both the unique and shared products provide beneficial information to the field, as of now, they are limited to those synthetic cannabinoids included in the current study. It cannot be said that other compounds or future generations will not produce equivalent pyrolytics. Further studies are necessary as well as synthesis of reference standards to confirm the products proposed. To reach a level of unambiguity of the identification of the proposed products, chromatographic analysis of reference standards as well as structural analysis via FTIR and NMR will be necessary.

Upon analysis of the studied cannabinoids, three major thermal degradation trends were apparent including: 1) a break on either side of the central carbonyl; 2) loss of the indole/indazole N-bonded substituent group; and 3) a ring size increase from indole/indazole to quinoline/cinnoline respectively. These trends may be used as a predictive model for other synthetic cannabinoids not studied here, not yet seen in casework, or for future generations yet to be synthesized.

The current study has set the stage for further investigation into the pyrolysis of synthetic cannabinoids as multiple products have been proposed, which could be utilized in various facets of forensics. Future steps are to quantify this process as to obtain an idea of the percent 
breakdown of these compounds that can be expected as well as investigating their presence in biological matrices. If they do in fact reach the body, they may produce toxic effects. The World Health Organization (WHO) has not classified the proposed products, but other indole, quinoline, cinnoline and naphthalene based compounds have been classified as group 2B, or possibly carcinogenic ${ }^{46}$.

\subsection{Conclusion}

The reported methodology was demonstrated as a suitable means for pyrolysis studies, and the performed analyses lay the groundwork for beginning to understand the effect of pyrolysis on synthetic cannabinoids. Ten pyrolytic products were observed in the herbal substrate trials, and fifty two pyrolytics were detected from the pyrolysis of the synthetic cannabinoids. The understanding of how the synthetic cannabinoids thermally degrade, via three observed breakdown or conversion trends, provides a predictive model to be used on additional compounds. The proposed pyrolytics also may provide additional analytes to be analyzed in toxicological samples and for use in toxicity studies.

\subsection{Funding}

This work was supported by the National Institute of Justice [2015-R2-CX-0032].

\subsection{References}

1. Brents, L. K.; Reichard, E. E.; Zimmerman, S. M.; Moran, J. H.; Fantegrossi, W. E.; Prather, P. L., Phase I hydroxylated metabolites of the K2 synthetic cannabinoid JWH-018 retain in vitro and in vivo cannabinoid 1 receptor affinity and activity. PloS one 2011, 6 (7), e21917. 2. Sobolevsky, T.; Prasolov, I.; Rodchenkov, G., Detection of JWH-018 metabolites in smoking mixture post-administration urine. Forensic science international 2010, 200 (1-3), 1417.

3. Wintermeyer, A.; Moller, I.; Thevis, M.; Jubner, M.; Beike, J.; Rothschild, M. A.; Bender, K., In vitro phase I metabolism of the synthetic cannabimimetic JWH-018. Analytical and bioanalytical chemistry 2010, 398 (5), 2141-53. 
4. Grigoryev, A.; Melnik, A.; Savchuk, S.; Simonov, A.; Rozhanets, V., Gas and liquid chromatography-mass spectrometry studies on the metabolism of the synthetic phenylacetylindole cannabimimetic JWH-250, the psychoactive component of smoking mixtures. Journal of chromatography. B, Analytical technologies in the biomedical and life sciences 2011, 879 (25), 2519-26.

5. $\quad$ Grigoryev, A.; Savchuk, S.; Melnik, A.; Moskaleva, N.; Dzhurko, J.; Ershov, M.; Nosyrev, A.; Vedenin, A.; Izotov, B.; Zabirova, I.; Rozhanets, V., Chromatography-mass spectrometry studies on the metabolism of synthetic cannabinoids JWH-018 and JWH-073, psychoactive components of smoking mixtures. Journal of chromatography. B, Analytical technologies in the biomedical and life sciences 2011, 879 (15-16), 1126-36.

6. $\quad$ Seely, K. A.; Brents, L. K.; Radominska-Pandya, A.; Endres, G. W.; Keyes, G. S.; Moran, J. H.; Prather, P. L., A major glucuronidated metabolite of JWH-018 is a neutral antagonist at CB1 receptors. Chemical research in toxicology 2012, 25 (4), 825-7.

7. Grigoryev, A.; Kavanagh, P.; Melnik, A.; Savchuk, S.; Simonov, A., Gas and liquid chromatography-mass spectrometry detection of the urinary metabolites of UR-144 and its major pyrolysis product. Journal of analytical toxicology 2013, 37 (5), 265-76.

8. $\quad$ Rajasekaran, M.; Brents, L. K.; Franks, L. N.; Moran, J. H.; Prather, P. L., Human metabolites of synthetic cannabinoids JWH-018 and JWH-073 bind with high affinity and act as potent agonists at cannabinoid type-2 receptors. Toxicology and applied pharmacology 2013, 269 (2), 100-8.

9. Wohlfarth, A.; Pang, S.; Zhu, M.; Gandhi, A. S.; Scheidweiler, K. B.; Liu, H. F.; Huestis, M. A., First metabolic profile of XLR-11, a novel synthetic cannabinoid, obtained by using human hepatocytes and high-resolution mass spectrometry. Clinical chemistry 2013, 59 (11), 1638-48.

10. Bell, S.; Nida, C., Pyrolysis of drugs of abuse: a comprehensive review. Drug testing and analysis 2015, 7 (6), 445-56.

11. Bellia, F.; La Mendola, D.; Pedone, C.; Rizzarelli, E.; Saviano, M.; Vecchio, G., Selectively functionalized cyclodextrins and their metal complexes. Chemical Society Reviews 2009, 38 (9), 2756-2781.

12. Auwarter, V.; Dresen, S.; Weinmann, W.; Muller, M.; Putz, M.; Ferreiros, N., 'Spice' and other herbal blends: harmless incense or cannabinoid designer drugs? Journal of mass spectrometry: JMS 2009, 44 (5), 832-7.

13. Fattore, L.; Fratta, W., Beyond THC: The New Generation of Cannabinoid Designer Drugs. Frontiers in behavioral neuroscience 2011, 5, 60.

14. Rosenbaum, C. D.; Carreiro, S. P.; Babu, K. M., Here today, gone tomorrow...and back again? A review of herbal marijuana alternatives (K2, Spice), synthetic cathinones (bath salts), kratom, Salvia divinorum, methoxetamine, and piperazines. Journal of medical toxicology : official journal of the American College of Medical Toxicology 2012, 8 (1), 15-32.

15. Deluca, P., Alert on New Recreational Drugs on the Web; Building Up a European-Wide Digital Web Scan-Monitoring System. Project, P. W. M., Ed. King's College: 2012.

16. Atwood, B. K.; Huffman, J.; Straiker, A.; Mackie, K., JWH018, a common constituent of 'Spice' herbal blends, is a potent and efficacious cannabinoid CB receptor agonist. British journal of pharmacology 2010, 160 (3), 585-93.

17. Vardakou, I.; Pistos, C.; Spiliopoulou, C., Spice drugs as a new trend: mode of action, identification and legislation. Toxicol Lett 2010, 197 (3), 157-62. 
18. Atwood, B. K.; Lee, D.; Straiker, A.; Widlanski, T. S.; Mackie, K., CP47,497-C8 and JWH073, commonly found in 'Spice' herbal blends, are potent and efficacious CB(1) cannabinoid receptor agonists. European journal of pharmacology 2011, 659 (2-3), 139-45. 19. Wiley, J. L.; Marusich, J. A.; Martin, B. R.; Huffman, J. W., 1-Pentyl-3phenylacetylindoles and JWH-018 share in vivo cannabinoid profiles in mice. Drug Alcohol Depend 2012, 123 (1-3), 148-53.

20. Toxicology, S. W. G. f. F., Standard Practices for Method Validation in Forensic Toxicology. Journal of analytical toxicology 2013, 37, 452-474.

21. Leonhart, M. M., Schedules of Controlled Substances; Temporary Placement of Five Synthetic Cannabinoids into Schedule I. Federal Register: Drug Enforcement Administration 2011, 76, 11075-11078.

22. Leonhart, M. M., Schedules of Controlled Substances: Placement of Five Synthetic Cannabinoids into Schedule I. Federal Register: Drug Enforcement Administration 2012, 77, 12508-12514.

23. EVERY- PALMER, S., Warning: legal synthetic cannabinoid- receptor agonists such as JWH- 018 may precipitate psychosis in vulnerable individuals. Addiction 2010, 105 (10), 18591860 .

24. Castellanos, D.; Singh, S.; Thornton, G.; Avila, M.; Moreno, A., Synthetic cannabinoid use: a case series of adolescents. The Journal of adolescent health : official publication of the Society for Adolescent Medicine 2011, 49 (4), 347-9.

25. Seely, K. A.; Lapoint, J.; Moran, J. H.; Fattore, L., Spice drugs are more than harmless herbal blends: a review of the pharmacology and toxicology of synthetic cannabinoids. Progress in neuro-psychopharmacology \& biological psychiatry 2012, 39 (2), 234-43.

26. Marshell, R.; Kearney-Ramos, T.; Brents, L. K.; Hyatt, W. S.; Tai, S.; Prather, P. L.; Fantegrossi, W. E., In vivo effects of synthetic cannabinoids JWH-018 and JWH-073 and phytocannabinoid Delta(9)-THC in mice: Inhalation versus intraperitoneal injection. Pharmacol. Biochem. Behav. 2014, 124, 40-47.

27. Seely, K. A.; Patton, A. L.; Moran, C. L.; Womack, M. L.; Prather, P. L.; Fantegrossi, W. E.; Radominska-Pandya, A.; Endres, G. W.; Channell, K. B.; Smith, N. H., Forensic investigation of K2, Spice, and "bath salt" commercial preparations: a three-year study of new designer drug products containing synthetic cannabinoid, stimulant, and hallucinogenic compounds. Forensic science international 2013, 233 (1-3), 416-422.

28. Kavanagh, P.; Grigoryev, A.; Savchuk, S.; Mikhura, I.; Formanovsky, A., UR-144 in products sold via the Internet: identification of related compounds and characterization of pyrolysis products. Drug testing and analysis 2013, 5 (8), 683-92.

29. Koller, V. J.; Zlabinger, G. J.; Auwarter, V.; Fuchs, S.; Knasmueller, S., Toxicological profiles of selected synthetic cannabinoids showing high binding affinities to the cannabinoid receptor subtype $\mathrm{CB}(1)$. Archives of toxicology 2013, 87 (7), 1287-97.

30. Favretto, D.; Pascali, J. P.; Tagliaro, F., New challenges and innovation in forensic toxicology: focus on the "New Psychoactive Substances". Journal of Chromatography A 2013, 1287, 84-95.

31. Arntson, A.; Ofsa, B.; Lancaster, D.; Simon, J. R.; McMullin, M.; Logan, B., Validation of a novel immunoassay for the detection of synthetic cannabinoids and metabolites in urine specimens. Journal of analytical toxicology 2013, 37 (5), 284-290. 
32. Meyer, M. R.; Maurer, H. H., Current status of hyphenated mass spectrometry in studies of the metabolism of drugs of abuse, including doping agents. Analytical and bioanalytical chemistry 2012, 402 (1), 195-208.

33. Hudson, S.; Ramsey, J., The emergence and analysis of synthetic cannabinoids. Drug testing and analysis 2011, 3 (7-8), 466-78.

34. Wohlfarth, A.; Weinmann, W., Bioanalysis of new designer drugs. Bioanalysis 2010, 2 (5), 965-979.

35. Centers, A. A. o. P. C., Synthetic Marijuana Data. 2014, 1-4.

36. Scientific Working Group for Forensic Toxicology (SWGTOX) - Recommendations of the Research, Development, Testing, and Evaluation Committee*. Journal of analytical toxicology 2013, 37 (3), 187-191.

37. AIT Among the First to Offer Blood Test for Synthetic Marijuana Compounds JWH-018 and JWH-073 (K2, Spice). 2-17-2011, 2011.

38. Penn, H. J.; Langman, L. J.; Unold, D.; Shields, J.; Nichols, J. H., Detection of synthetic cannabinoids in herbal incense products. Clinical biochemistry 2011, 44 (13), 1163-5.

39. Daw, R. C.; Grabenauer, M.; Pande, P. G.; Cox, A. O.; Kovach, A. L.; Davis, K. H.; Wiley, J. L.; Stout, P. R.; Thomas, B. F., Pyrolysis studies of synthetic cannabinoids in herbal products. Drug Alcohol Depend. 2014, 140, e44.

40. Kanamori, T.; Kanda, K.; Yamamuro, T.; Kuwayama, K.; Tsujikawa, K.; Iwata, Y. T.; Inoue, H., Detection of main metabolites of XLR-11 and its thermal degradation product in human hepatoma HepaRG cells and human urine. Drug testing and analysis 2015.

41. Information, N. C. f. B., Quinoline. 09-16-2004 ed.; PubChem Compound Database.

42. Information, N. C. f. B., Indole. 09-16-2004 ed; PubChem Compound Database.

43. Information, N. C. f. B., Naphthalene. 03-26-2005 ed.; PubChem Compound Database.

44. Information, N. C. f. B., 1-Butyl-3-(1-naphthoyl)indole. 10-25-2006 ed.; PubChem Compound Database.

45. Information, N. C. f. B., 1-Pentyl-3-(1-naphthoyl)indole. 10-25-2006 ed.; PubChem Compound Database.

46. IARC Monographs on the Evaluation of Carcinogenic Risks to Humans. World Health Organization: International Agency for Research on Cancer. 


\section{Chapter 3: Utilization of Novel Pyrolysis Methodology to Evaluate the Thermal Degradation Products of Classical Drugs of Abuse}

Submitted to the Journal of Forensic Science on March 7, 2018. Utilization of Novel Pyrolysis Methodology to Evaluate the Thermal Degradation Products of Classical Drugs of Abuse. S. Raso and S. Bell, Journal of Forensic Science.

Permission will be obtained from the co-authors and the Journal of Forensic Science according to the journal's license's, copyright and permissions policy upon acceptance. A copy of the permissions agreement will be shown in Appendix A if obtained before submission to the college.

Smoking as a route of ingestion of drugs of abuse is still common amongst users, and this poses numerous health risks both to the users and bystanders. It is understood that drug compounds thermally degrade during the smoking process, but knowledge of the effects of such products is still lacking. This report employs a previously optimized in-house method to consolidate previously unconfirmed pyrolytic results as well as identify newly observed products of drugs including cocaine, heroin and phenethylamines. This methodology has already demonstrated its ability to evaluate the pyrolysis process with a number of synthetic cannabinoid compounds, and the current opioid crisis led to the movement back to the classical drugs. In the 1980's, a type of encephalopathy specifically seen in heroin smokers was coined heroin induced spongiform leukoencephalopathy or HSL. As this effect had a larger observance in smokers versus injectors, hypotheses of the produced thermal degradants having additional pharmacodynamic effects arose. After analysis, different previously detected as well as newly observed degradants were identified. Interestingly, some of the products are even metabolic products, and the impact the identified products may have span across drug investigations, toxicological assessment, metabolic evaluations and public health. 


\subsection{Introduction}

A critical review of thermal degradation studies of drugs of abuse was published by our group in $2015^{1}$. The impetus for this project was to consolidate findings to date related to drugs of abuse such as heroin and cocaine and to provide a foundation for work regarding novel psychoactive substances (NPSs), primarily synthetic cannabinoids. The fact that these compounds are ingested primarily by smoking (and now "vaping") coupled with the constellation of toxic effects observed has driven this interest. As examples, XLR-11 and UR144 both contain a cyclopropyl group that opens with heating to form known degradation products referred to as degradants which are detectable in toxicological samples ${ }^{2-5}$. It is also observed that these degradants produce a metabolic profile separate from the parent compound ${ }^{3}$.This renewed interest in thermal degradation products has benefited from improved instrumental capability over what was available even fifteen years ago. Accordingly, the goal of this project was to revisit earlier thermal degradation studies of drugs such as cocaine, heroin, and phenethylamines using optimized experimental conditions and designs coupled with improved instrumental detection methods.

While greater attention is being paid to NPSs, these "older" drugs remain a forensic, legal, and public health challenge. Heroin and related synthetic opioids have become the current greatest overdose threat. The National Institute of Health (NIH) testified to this issue in front of the senate, stating a current heroin use population of nearly 1 million $^{6-7}$. Unlike synthetic cannabinoids, opioids are ingested by injection, snorting, and smoking, but issues related to smoking and thermal degradation products has long been a concern. Beginning in the 1980's, it was observed that heroin users that smoked the drug had higher mortality rates due to a type of encephalopathy called heroin induced spongiform leukoencephalopathy, HSL, which was not 
seen in users who instead utilized injection ${ }^{8-26}$. This led to initial investigations into the pyrolytic process of smoking illicit drugs and tentative identification of any thermal degradation products.

The current investigation employed a proven in-house method to revisit the pyrolysis of commonly smoked illicit drugs and demonstrate the production of both previously observed thermal degradation products as well as several newly identified products ${ }^{27}$. The findings are interpreted in the context of current understanding related to the potential toxicity of the thermal degradation products and effect on metabolite presence interpretation.

\subsection{Methods}

\subsubsection{Materials and Reagents}

An apparatus to carry out controlled pyrolysis experiments was constructed in-house and specifications previously reported $^{27}$. Sample collection utilized $5 \mathrm{~mL}$ LUER-SLIP plastic syringes and $17 \mathrm{~mm}, 0.45 \mu \mathrm{m}$ syringe filters from Thermo Fisher Scientific (Waltham, MA) with methanol as the solvent from Fisher Scientific (Hampton, NH). Standard solids of methamphetamine, cocaine, heroin, and fentanyl were obtained from Sigma-Aldrich (St. Louis, MO).

\subsubsection{Pyrolysis Trials}

Solid reference standards were analyzed individually by being placed directly into the reaction zone of the previously described apparatus. No additional sample preparation was required. The reaction zone is enclosed with quartz wool to contain the pyrolysis of the drug while constant air flow was controlled with the vacuum pump. The pyrolysis study of each compound was conducted in replicates of 5 with each experiment utilizing $20 \mathrm{mg}$ of reference standard. Two additional experiments were carried out with a mixture of heroin and fentanyl 
given the current increase in fentanyl/heroin street samples. These mixtures were prepared to be $50 \% \mathrm{w} / \mathrm{w}$ of each compound.

\subsubsection{Sample Collection}

After heating, three samples were collected from different locations in the apparatus as previously described ${ }^{27}$. Samples were evaporated under nitrogen and reconstituted in fresh methanol. The capture solvent was initially reconstituted to $10 \mathrm{~mL}$, transferred to a $10 \mathrm{~mL}$ vial, re-evaporated and reconstituted to a final volume of $2 \mathrm{~mL}$. The rinse from the tube was also reconstituted to a final volume of $2 \mathrm{~mL}$, while the quartz/ashes sample was reconstituted to $500 \mu \mathrm{L}$. Each solid sample resulted in solutions, one from each location and for each drug, 5 individual samples were subjected to thermal degradation.

\subsubsection{Instrumental Analysis}

Analyses were performed using a Perkin Elmer Clarus 500 gas chromatograph with a Restek Rxi ${ }^{\circledR}-5$ Sil with Integra-Guard ${ }^{\circledR} 30 \mathrm{~m}$ x $250 \mu \mathrm{m} \times 0.5 \mu \mathrm{m}$ column coupled with a Clarus SQ8T mass spectrometer. Helium was used as the carrier gas with a flow of $12.1 \mathrm{~mL} / \mathrm{min}$. The injection volume was a splitless $2 \mu \mathrm{L}$ with the heater set at $275^{\circ} \mathrm{C}$. The gas chromatography (GC) method had an initial temperature of $100^{\circ} \mathrm{C}$, ramp of $20^{\circ} \mathrm{C} / \mathrm{min}$ and was held at $320^{\circ} \mathrm{C}$ for $2 \mathrm{~min}$ for a total run time of $13 \mathrm{~min}$. The mass spectrometer was set with a $1.00 \mathrm{~min}$ solvent delay, scan time of $0.2 \mathrm{~s}$ and a range of $40-500 \mathrm{~m} / \mathrm{z}$.

\subsubsection{Data Analysis}

The first step in the analysis scheme was selection of which chromatographic peaks to consider. Only those peaks that were consistently present in at least three of the five trials were selected for further evaluation. A library search using the National Institute of Standards and 
Technology (NIST/NIH) database was carried out to obtain a match or a starting point of general structure for mass spectrum breakdown analysis. This mass spectrum analysis was conducted to insure the fragment breakdown was plausible for the predicted pyrolytic compound, and the chromatography was evaluated via retention index (RI) where possible. This combined data analysis along with a logical mechanism of production via pyrolysis was used to predict compound identifications, and these were confirmed with reference materials where available.

\subsection{Results}

\subsubsection{Methamphetamine}

The pyrolysis of methamphetamine produced five products Figure 3.1. Among these products are both previously found and newly observed pyrolytics. The amphetamine was confirmed with reference standard, while the others were identified by an obtained NIST database match of at least 75 and reasonable mass spectrum analysis. Amphetamine, $\mathrm{N}$-formylmethamphetamine and $\mathrm{N}, \mathrm{N}$-dimethyl-amphetamine were identified in previous studies spanning from the late 80 's into the 2000 's, which are described in the review paper by Nida and Bell ${ }^{1}$. Amphetamine is a central nervous stimulant as well as N,N-dimethylamphetamine, which may produce an increased effect on the user when smoking methamphetamine. Pharmacodynamic information of $\mathrm{N}$-formyl-methampetamine is lacking and in need of investigation. On the other hand, $\mathrm{N}$-acetyl-methamphetamine and N,N-dimethyl-phenethylamine have not previously been reported as thermal degradation products of methamphetamine. The acetylated product, compound 4 from Figure 1, has been thought to be an impurity in illicit drug synthesis, but now appears to be the product of a pyrolytic acetylation process, which could further break down into previously reported compounds, 1,2 and $3^{28}$. Breakdown of compound 2 could lead to the production of $\mathrm{N}, \mathrm{N}$-dimethyl-phenethylamine via demethylation, which is known to be a 


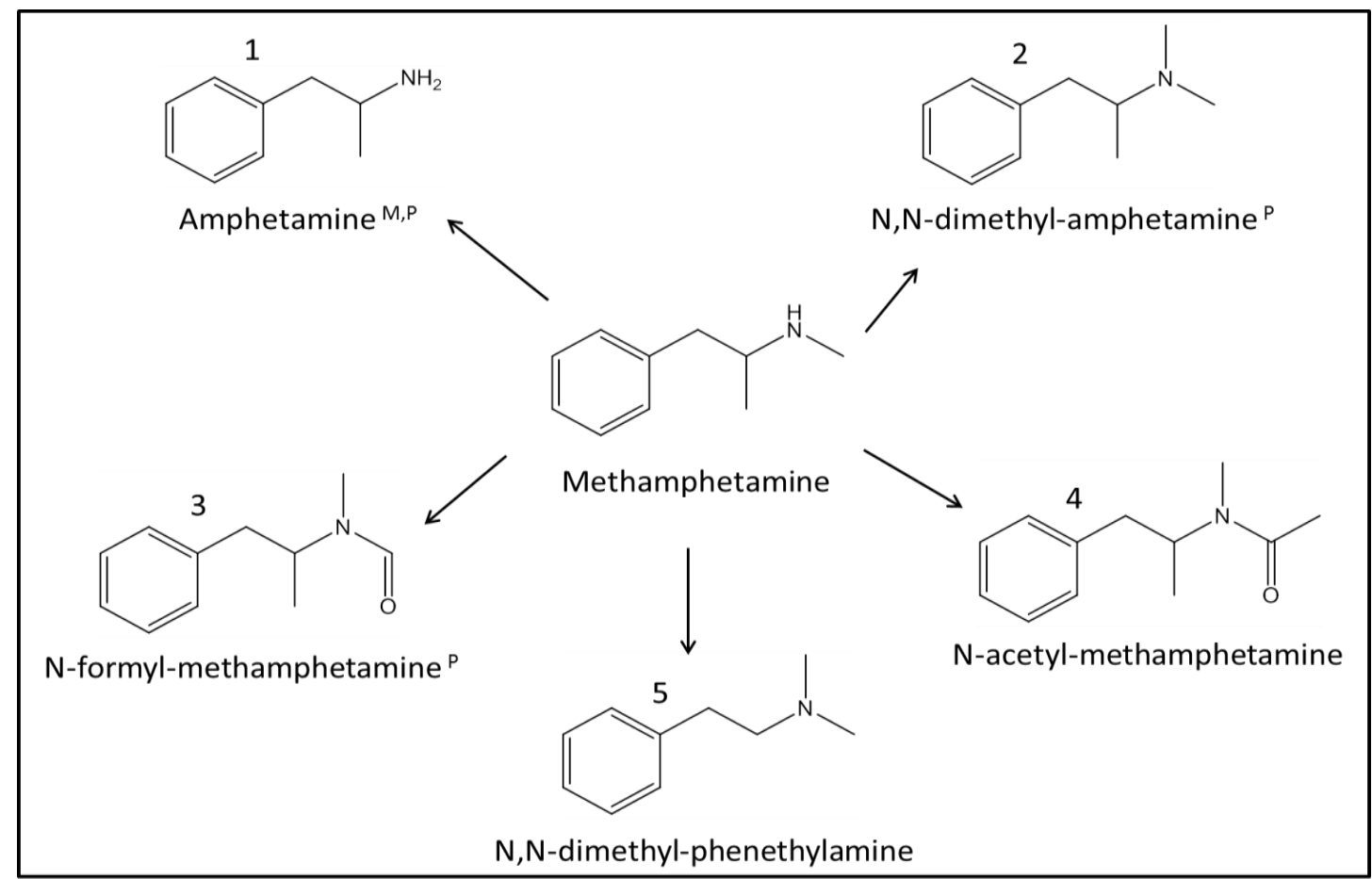

Figure 3.1 - Proposed pyrolytic breakdown of methamphetamine into five pyrolytic products. $\mathrm{M}=$ Methamphetamine metabolite; $\mathrm{P}=$ Previously found pyrolytic product stimulant and mood enhancer used as a flavoring agent. The production of N,N-dimethylphenethylamine through the smoking process could enhance the effect and thus increase the potential for dependence and addition. These compounds would also follow their own metabolic and excretion pathways. The para- location on the benzene ring is a common location for possible hydroxylation between each structure, but the additional methyl, aldehyde or ketone groups in compounds 2-5 may produce further binding properties as well as supplementary phase I and II reaction sites. These metabolites would be missed under current assays, as their ion transitions would differ from commonly monitored transitions in methamphetamine urine analysis.

\subsubsection{Cocaine}

In the late 80 's, cocaine pyrolysis began to be examined and two major products identified were benzoic acid and anhydroecgonine methyl ester, AEME ${ }^{29-30}$. The current study 


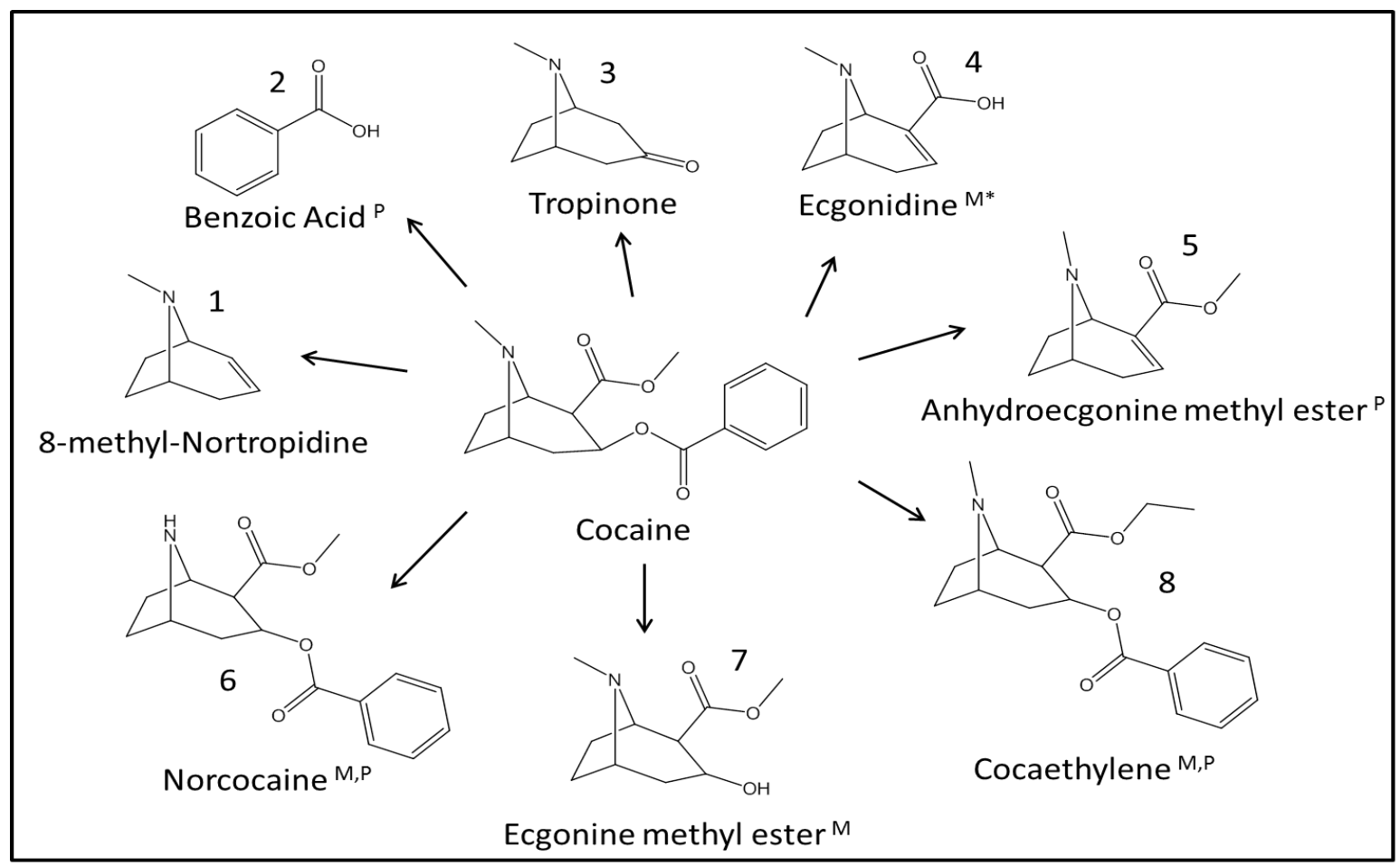

Figure 3.2 - Proposed pyrolytic breakdown of cocaine into eight pyrolytic products. $\mathrm{M}=$ Cocaine metabolite; $\mathrm{P}=$ Previously found pyrolytic; $\mathrm{M}^{*}=$ Metabolite of a previously reported cocaine pyrolytic product

also observed and confirmed these two pyrolytic products, while the others were identified using the NIST database and mass spectrum analysis. Compound 1 is a predicted product based on an equivalent mass spectrum of tropinone except for the parent peak being $17 \mathrm{Da}$ smaller. This difference could be explained by the loss of the carbonyl group of tropinone to produce compound 1. Along with AEME and benzoic acid, two other reported degradants from a 2007 study were present, cocaethylene and norcocaine ${ }^{31}$. AEME has been reported to induce greater neurotoxicity than cocaine in a 2012 study, which indicates that other pyrolytics may also produce added acute toxic effects compared to the parent drugs ${ }^{32}$. Cocaethylene and norcocaine have been reported as biomarkers in hair for cocaine abuse, but not directly linked to abuse by smoking ${ }^{33-34}$. Four previously unreported products were detected in the current study, which are outlined in Figure 3.2. Of the newly reported products, two tropinone compounds, 1 and 3, are 
possibly further degradations of earlier observed products. Tropinone is a precursor to atropine, an efficacious medication for some nerve agents, and its toxicology is not entirely understood, though is considered a level 2 health risk $^{35}$. Compound 1, 8-methyl-nortropidine, is a reduction of tropinone, whose pharmacodynamics effects are unknown. Ecgonine methyl ester, another newly discovered pyrolytic product is typically seen as a metabolite of cocaine along with a number of the other detected products, including compounds 4 and 5. Ecgonidine has been described as metabolic product of the pyrolytic product, AEME, and thus has been useful as a marker for inhalation as the mode of ingestion when present ${ }^{36}$. The current study supports that ecgonidine is a useful smoking marker, but it now may be present even without the necessary breakdown of other products. The degradants shown here all have multiple sites for metabolic processes, and extensive studies would be necessary to identify possible metabolites. More importantly, the numerous products observed provide a basis for toxicological assays and target compounds in the analysis of drug paraphernalia.

\subsubsection{Heroin}

Extensive studies on heroin pyrolysis were conducted in the late 80's and revisited in the early 2000's. In the 80 's, four predicted pyrolytic products were observed including compounds 2, 3 and 7 from Figure $3.3^{37}$. These three compounds are produced via acetylation processes at the amine chain and phenanthrene ring. Most notable is 6-monoacetylmorphine (6-MAM), which is the major metabolite of heroin and the targeted marker for the use of heroin in comparison to morphine. The remaining two have largely been unstudied and unconfirmed, but here, a proposed GC/MS spectral fragmentation pattern of compound 2 is shown in Figure 3.4. Sequential deacetylation and dehydration steps are predicted at steps A - D2. At steps D1, E and $\mathrm{F}$, the breakdown of the ethanamine side chain takes place. The $236 \mathrm{~m} / \mathrm{z}$ species is a predicted 
intermediate produced as the dehydrogenation of the position 9 hydrogen of the phenanthrene ring and the ethanamine side chain cleavage is initiated. Compounds 1, 4, 5 and 7 were able to be confirmed with reference standards.

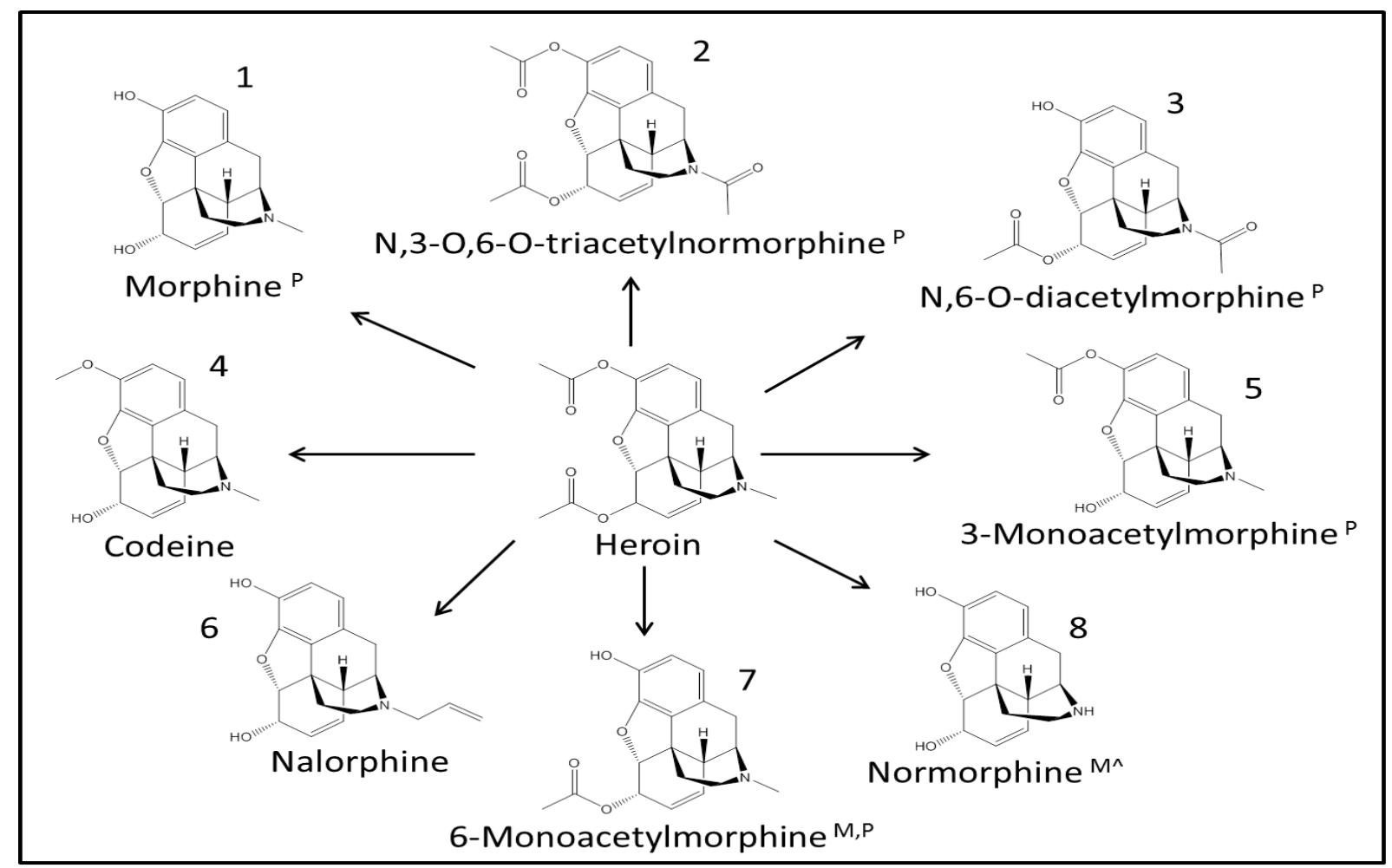

Figure 3.3 - Proposed pyrolytic breakdown of heroin into eight pyrolytic products. $\mathrm{M}=$ Heroin metabolite; $\mathrm{P}=$ Previously found pyrolytic; $\mathrm{M}^{\wedge}=$ Metabolite of a newly reported pyrolytic product of heroin

Brenneisen and Hasler studied heroin pyrolysis through the inhalation method commonly termed "smoking the dragon", where they observed similar products including another major breakdown compound, morphine ${ }^{38}$. In addition to these previously detected products, three additional products were observed including two tentatively identified: normorphine and nalorphine, and one confirmed: codeine. Normorphine is the major metabolic product of morphine, but has little opioid activity ${ }^{39}$. On the other hand, nalorphine is a mixed agonistantagonist at the opioid receptors, and produces side effects such as dysphoria, anxiety and hallucinations ${ }^{40-41}$. The third newly observed pyrolytic is codeine. A significant consequence of 
the observed opioid thermal degradants is the vast difference in half-lives in comparison to heroin. Heroin has a rapid half-life of a few minutes, but the thermal degradants may lead to a

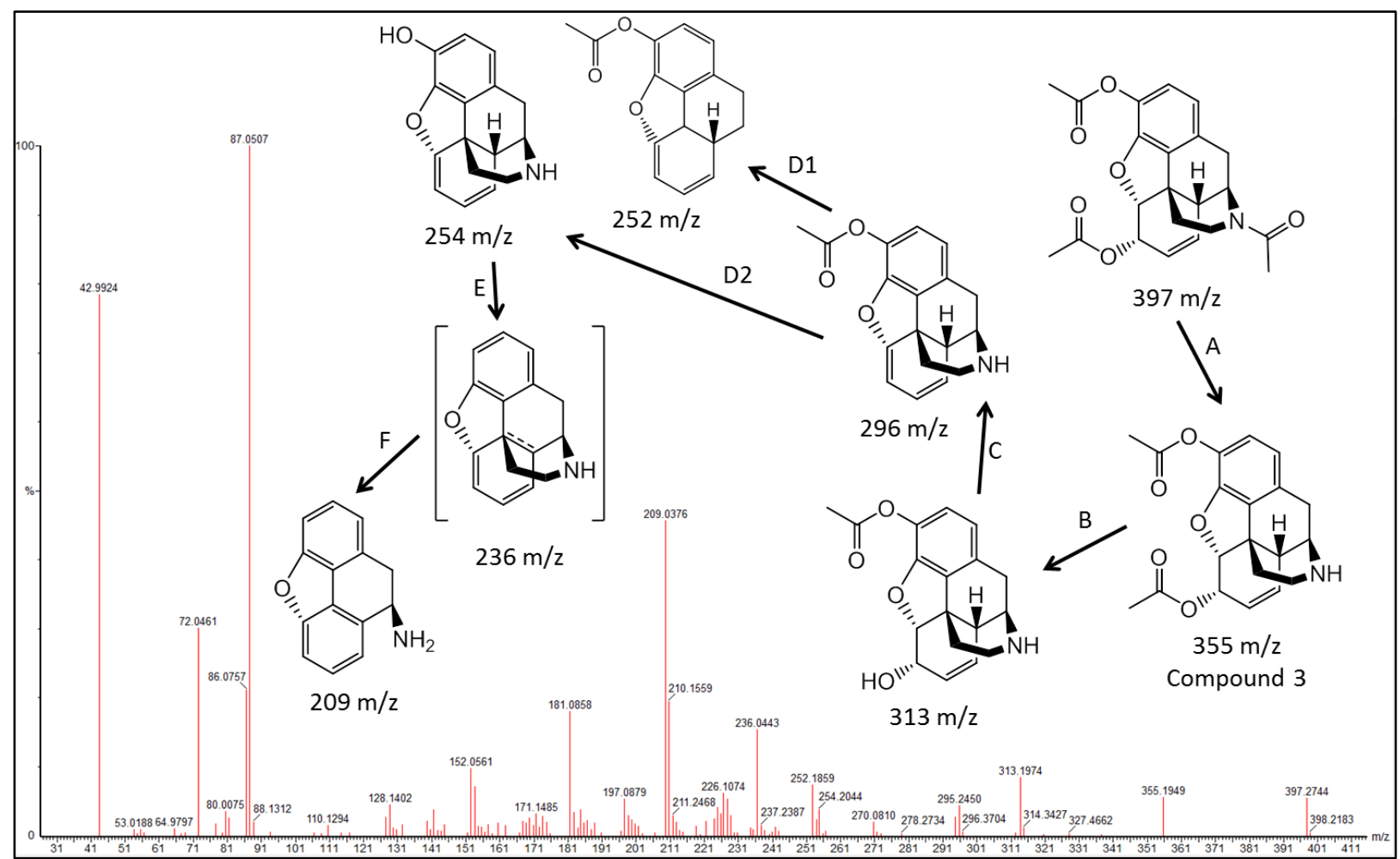

Figure 3.4 - Proposed fragmentation pattern of N,3-O,6-O-triacetylnormorphine, compound 2 from Figure 3.3.

longer duration of action as the additional opiate breakdown compounds, such as nalorphine, codeine and morphine can have half-lives of multiple hours ${ }^{42-44}$.

\subsubsection{Fentanyl}

In the late 90's, fentanyl, an extremely potent opiate, become a popular drug of abuse and has recently been a large part of the opioid epidemic with its lacing of heroin street samples. During the 2000's, a few pyrolysis studies were conducted, which determined that fentanyl is thermally stable up to approximately $500^{\circ} \mathrm{C}$, but at higher temperatures, pyrolytic products began to be observed ${ }^{45-46}$. In Figure 3.5, there are a number of these products that were also identified in the current study, shown as compounds 1,2, 4 and 6, with 2 and 6 being confirmed. 
Despropionyl fentanyl is also a metabolic product of fentanyl as well as a common precursor in fentanyl synthesis, but the pharmacologic activity of this compound is thought to be lower than that of the parent ${ }^{47}$. Another earlier noted compound also observed here is 1-phenethylpyridium (1-PEP), though has not been confirmed via reference standard in any of the studies ${ }^{46}$. The next consistently observed pyrolytic between studies is compound 2, N-phenyl-propanamide, which is also known as propionanilide. Propionanilide has been reported as a possibly harmful agent if orally consumed as noted by the international Globally Harmonized System of Classification and Labelling of Chemicals (GHS) ${ }^{48}$. Two newly found pyrolytics were tentatively identified and include the methyl ester of propionanilide and bibenzyl. GHS labels bibenzyl as a category 1 chemical if entered into airways, which is a pertinent danger to any user and bystanders who may inhale the vapors ${ }^{49}$. The last major product observed was norfentanyl, which is both a previously

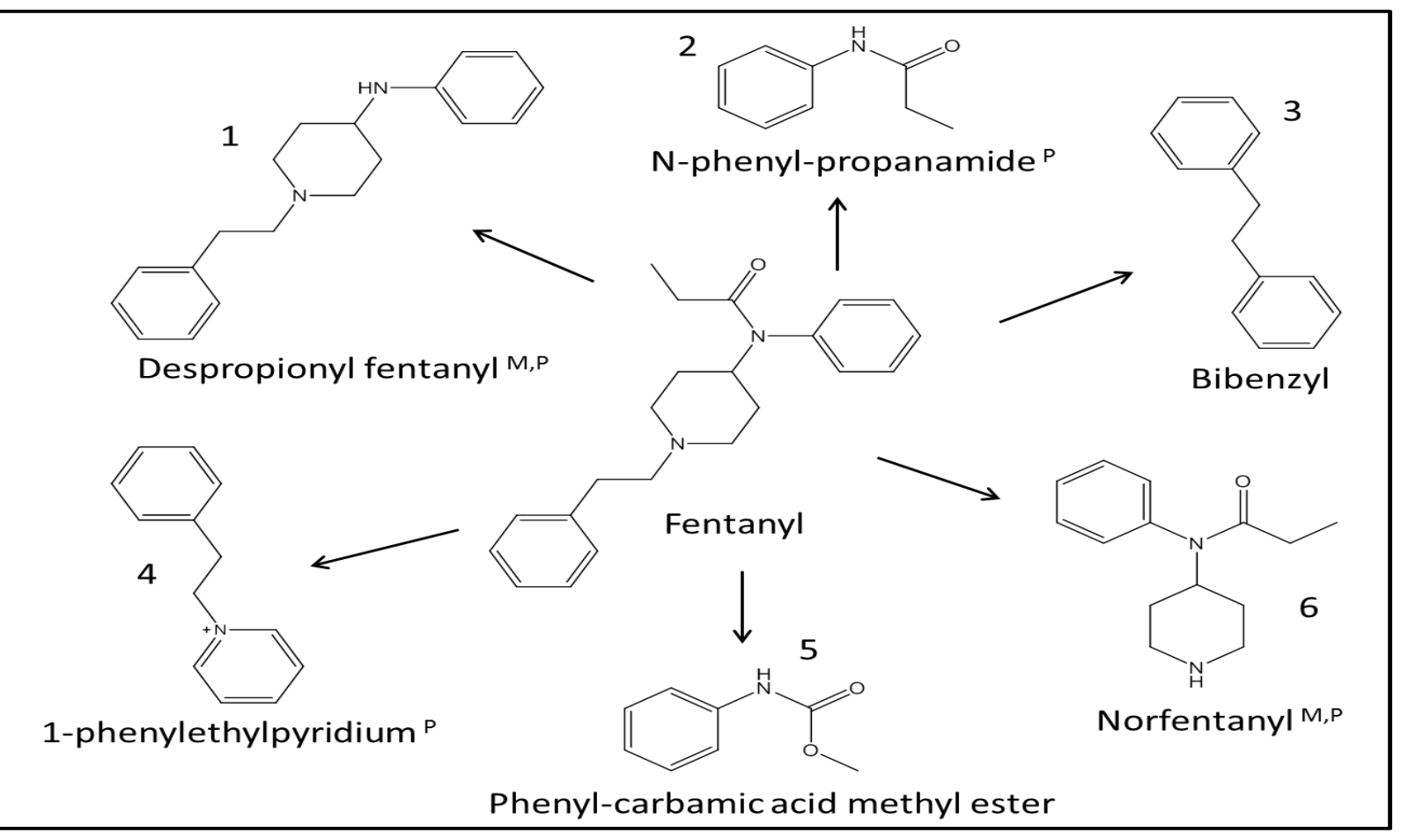

Figure 3.5 - Proposed pyrolytic breakdown of fentanyl into six pyrolytic products. $\mathrm{M}=$ Fentanyl metabolite; $\mathrm{P}=$ Previously found pyrolytic 
detect pyrolytic as well as an observed metabolic product of fentanyl ${ }^{1}$. It has been reported that the ratio of fentanyl to norfentanyl can be beneficial in determining if there was an acute fentanyl toxicity or chronic fentanyl utilization ${ }^{50}$. This ratio is believed to be $>8$ for acute toxicity, but this value is based on a route of ingestion other than smoking. This would need to be evaluated with smoking to ensure confidence in diagnosing acute toxicity in such instances. This is just one of numerous areas of impact where fully understanding the smoking process is necessary.

\subsection{Discussion}

Due to differing toxic effects seen in users of the same drug but with different routes of ingestion, it had been hypothesized in the past that the abuse of a drug may become more toxic when smoked in comparison to other routes of ingestion. An example of this is HSL and had been briefly examined. Research in this area had begun to lose interest due to an absence of necessary instrumentation capabilities and limited results. The idea has been rejuvenated with an investigation of the pyrolysis with synthetic cannabinoids as their acute toxicity is not understood, and led to a revisit of these still abused classical drugs. The production and ingestion of additional compounds unbeknownst to the user could be a cause of the further toxicity, and a complete understanding of the scope of thermal degradation is vital. The knowledge of the presence of specific pyrolytic products allows for toxicity evaluations, a larger analyte pool for drug investigations and novel metabolic investigations, which may affect current timelines used to estimate dosage events.

The first impact to consider is the pharmacologic effects and toxicity the identified products may induce. This concept is supported by the high mortality rate of heroin smokers due to HSL in the 1980's. As the thermal degradation products are identified, the evaluation of their toxicity and their individual impacts can be researched. The thermal degradants can be smaller in 
size and more lipophilic, which both could increase the compounds ability to enter the blood stream and interact with receptors throughout the body. The same receptors as the parent drug may be the sites of action, but depending on the structure of the degradant, they may interact differently and in new sites. Studies on the prevalence of the thermal degradants to be ingested, binding affinity evaluations and toxicological assays are interesting avenues of research that stem from the knowledge of the re-confirmed and newly identified products.

Another advantage of knowing these products to consider is within drug investigations. Drug paraphernalia collected as evidence can now be further processed. If a drug was smoked in set paraphernalia, it is unlikely that the parent compound would remain, but the thermal degradants may be left behind. After this report, more analytical targets are available for the processing of such evidence with simple collection and analysis techniques that common laboratories could implement. Detection of the pyrolytic products may give investigators a starting point for their investigations, and may lead to the request of the appropriate toxicological testing, as not all precincts may have the monetary ability for wide spectrum testing. Having additional information at their disposal, more options will be available to use to decipher appropriate actions and conclusions within both criminal and public health cases.

An interesting finding is the presence of metabolic products as thermal degradation products. This can have a resounding effect on the interpretation of toxicological assays. Metabolites are typically used as markers of a parent drug and based on studied pharmacology, a dosage time and concentration can be extrapolated. However, if these products are produced at the dosage event via smoking, this affects how those interpretations can be made. Secondly, the thermal degradants open the possibility for additional sites for phase I and II metabolism, and will have unique metabolic products. As metabolic profiles can be established for the thermal 
degradants, these metabolites may be detected for a longer time period and be used as additional markers for the smoking of a drug compound. The pharmacologic data of the thermal degradants will have to be added to current knowledge of parent drugs for accurate dosage estimates as well, as the concentration of known metabolites is no longer zero, but the amount of the compound as a pyrolytic that is ingested along with the parent.

\subsection{Conclusion}

The current study has reconfirmed the production of many thermal degradants that had been previously reported as well has detected a number of newly observed pyrolytics using the methodology previously reported. The observed products can be useful in analyzing drug paraphernalia, provide novel target analytes in toxicological assays and affect the interpretation of currently monitored metabolites. As more products are identified, numerous research opportunities will arise to understand the rate of ingestion, pharmacology, metabolism and toxicity of each thermal degradant.

\subsection{Funding}

This work was supported by the National Institute of Justice [2015-R2-CX-0032].

\subsection{References}

1. Bell, S.; Nida, C., Pyrolysis of drugs of abuse: a comprehensive review. Drug testing and analysis 2015, 7 (6), 445-56.

2. Adamowicz, P.; Zuba, D.; Sekula, K., Analysis of UR-144 and its pyrolysis product in blood and their metabolites in urine. Forensic science international 2013, 233 (1-3), 320-7.

3. Kanamori, T.; Kanda, K.; Yamamuro, T.; Kuwayama, K.; Tsujikawa, K.; Iwata, Y. T.; Inoue, H., Detection of main metabolites of XLR-11 and its thermal degradation product in human hepatoma HepaRG cells and human urine. Drug testing and analysis 2015.

4. Thomas, B. F.; Lefever, T. W.; Cortes, R. A.; Grabenauer, M.; Kovach, A. L.; Cox, A. O.; Patel, P. R.; Pollard, G. T.; Marusich, J. A.; Kevin, R. C.; Gamage, T. F.; Wiley, J. L., Thermolytic Degradation of Synthetic Cannabinoids: Chemical Exposures and Pharmacological Consequences. The Journal of pharmacology and experimental therapeutics 2017, 361 (1), 162171. 
5. Kavanagh, P.; Grigoryev, A.; Savchuk, S.; Mikhura, I.; Formanovsky, A., UR-144 in products sold via the Internet: identification of related compounds and characterization of pyrolysis products. Drug testing and analysis 2013, 5 (8), 683-92.

6. World Drug Report. UNODC: 2017.

7. The Federal Response to the Opioid Crisis. www.drugabuse.gov/about-nida/legislativeactivities/testimony-to-congress/2017/federal-response-to-opioid-crisis.

8. John Strang, P. G. a. M. G., Heroin smoking by 'chasing the dragon': origins and history. Addiction 1997, 92 (6), 673-683.

9. Nicolo Rizzuto, M. M., Sergio Ferrari, Tiziana Cavallaro, Marco Sparaco, Giulio Boso and Luigi Gaetti, Delayed spongiform leukoencephalopathy after heroin abuse. Acta neuropathologica 1997, 94, 87-90.

10. W. Weber, H. H., P. Moller, K. Bade and D. Kuhne, Toxic spongiform

leucoencephalopathy after inhaling heroin vapour. Eur. Radiol. 1998, 8, 749-755.

11. Michael D. Hill, P. W. C. a. J. R. P., Chasing the dragon - neurological toxicity associated with inhalation of heroin vapour: case report. Canadian Medical Association 2000, 162 (2), 236-238.

12. Blair, A. H. R. a. R., Symmetric Deep Cerebellar Lesions After Smoking Heroin. Arch Neurol 2003, 60, 1605-1606.

13. Ciaran F. Keogh, G. T. A., Sian D. Spacey, Kevin E. Forkheim and Douglas A. Graeb, Neuroimaging Features of Heroin Toxicity: "Chasing the Dragon". American Roentgen Ray Society 2003, 180, 847-850.

14. Gacouin, A.; Lavoue, S.; Signouret, T.; Person, A.; Dinard, M. D.; Shpak, N.; Thomas, R., Reversible spongiform leucoencephalopathy after inhalation of heated heroin. Intensive Care Med 2003, 29 (6), 1012-1015.

15. Heales, S.; Crawley, F.; Rudge, P., Reversible parkinsonism following heroin pyrolysate inhalation is associated with tetrahydrobiopterin deficiency. Mov Disord 2004, 19 (10), 1248-51.

16. P.J. Egan, F. W. B. a. F. S., Spongiform Leucoencephalopathy After Inhaling Illicit Heroin and Due to Carbonmonoxide-Intoxication. Fortschr Neurol Psychiat 2004, 72, 26-35.

17. Bartlett, E.; Mikulis, D. J., Chasing "chasing the dragon" with MRI: leukoencephalopathy in drug abuse. Br J Radiol 2005, 78 (935), 997-1004.

18. Blasel, S.; Hattingen, E.; Adelmann, M.; Nichtweiss, M.; Zanella, F.; Weidauer, S., Toxic leukoencephalopathy after heroin abuse without heroin vapor inhalation: MR imaging and clinical features in three patients. Clin Neuroradiol 2010, 20 (1), 48-53.

19. Jane A Buxton, R. S., Lorne Clearsky, Natalie Angus, Lena Shah and Marcus Lem, Chasing the dragon - characterizing cases of leukoencephalopathy asspciated with heroin inhalation in British Columbia. Harm reduction journal 2011, 8 (3), 1-5.

20. J. Hagel, G. A., T. Vertinsky, M.K. Heran and C. Keogh, "Chasing the dragon" - imaging of heroin inhalation leukoencephalopathy. Can Assoc Radiol J 2005, 56 (4), 199-203.

21. H. Long, K. D., R.S. Hoffman and L.S. Nelson, A fatal case of spongiform leukoencephalopathy linked to "chasing the dragon". J Toxicol Clin Toxicol 2003, 41 (6), 887891.

22. Waespe, H. H. a. W., Leukoencephalopathy following inhalation of heroin pyrolysate. Schweiz Med Wochenschr 1990, 120 (48), 1801-1805.

23. T.P. Tan, P. R. A., J. Valk and E.C. Wolters, Toxic leukoencephalopathy after inhalation of poisoned heroin: MR findings. AJNR Am J Neuroradiol 1994, 15 (1), 175-178. 
24. Y.J. Chang, C. H. T. a. C. J. C., Leukoencephalopathy after inhalation of heroin vapor. $J$ Formos Med Assoc 1997, 96 (9), 758-760.

25. D. Nuytten, E. W., K. Michiels, M. Ferrante, H. Verbraeken, R. Daelemans, E. Baeck and P. Cras, Drug-induced spongiform leucoencephalopathy, a case report with review of the literature. Acta Neurol Belg 1998, 98 (1), 32-35.

26. N. Sodeyama, S. O., T. Oka, K. Nagashima, E. Ozawa and H. Mizusawa, A Novel Form of Reversible Subacute Spongiform Leukoencephalopathy. European Neurology 2001, 46, 223 224.

27. Raso, S.; Bell, S., Qualitative Analysis and Detection of the Pyrolytic Products of JWH018 and 11 Additional Synthetic Cannabinoids in the Presence of Common Herbal Smoking Substrates. Journal of analytical toxicology 2017, 41 (6), 551-558.

28. Dayrit, F. M.; Dumlao, M. C., Impurity profiling of methamphetamine hydrochloride drugs seized in the Philippines. Forensic science international 2004, 144 (1), 29-36.

29. Martin, B. R.; Lue, L. P.; Boni, J. P., Pyrolysis and volatilization of cocaine. Journal of analytical toxicology 1989, 13 (3), 158-162.

30. Ishigami, Y. N. a. A., Inhalation Efficiency of Free-Base Cocaine by Pyrolysis of 'Crack' and Cocaine Hydrochloride. Journal of analytical toxicology 1991, 15, 105-109.

31. Gayton-Ely, M.; Shakleya, D. M.; Bell, S. C., Application of a pyroprobe to simulate smoking and metabolic degradation of abused drugs through analytical pyrolysis. Journal of forensic sciences 2007, 52 (2), 473-8.

32. Garcia, R. C.; Dati, L. M.; Fukuda, S.; Torres, L. H.; Moura, S.; de Carvalho, N. D.; Carrettiero, D. C.; Camarini, R.; Levada-Pires, A. C.; Yonamine, M.; Negrini-Neto, O.; Abdalla, F. M.; Sandoval, M. R.; Afeche, S. C.; Marcourakis, T., Neurotoxicity of anhydroecgonine methyl ester, a crack cocaine pyrolysis product. Toxicological sciences : an official journal of the Society of Toxicology 2012, 128 (1), 223-34.

33. Fiorentin, T. R.; Scherer, J. N.; Marcelo, M. C. A.; Sousa, T. R. V.; Pechansky, F.; Ferrão, M. F.; Limberger, R. P., Comparison of Cocaine/Crack Biomarkers Concentrations in Oral Fluid, Urine and Plasma Simultaneously Collected From Drug Users. Journal of analytical toxicology 2017, 1-8.

34. Gambelunghe, C.; Rossi, R.; Aroni, K.; Gili, A.; Bacci, M.; Pascali, V.; Fucci, N., Norcocaine and cocaethylene distribution patterns in hair samples from light, moderate, and heavy cocaine users. Drug testing and analysis 2017, 9 (2), 161-167.

35. Tropinone. https://pubchem.ncbi.nlm.nih.gov/compound/Tropinone\#section=Top.

36. Fandiño, A. S.; Toennes, S. W.; Kauert, G. F., Studies on Hydrolytic and Oxidative Metabolic Pathways of Anhydroecgonine Methyl Ester (Methylecgonidine) Using Microsomal Preparations from Rat Organs. Chemical research in toxicology 2002, 15 (12), 1543-1548. 37. Brine, C. E. C. a. D. R., Pyrolysis products of heroin. Journal of forensic sciences 1985, 30 (1), 251-261.

38. Hasler, R. B. a. F., GC/MS determination of pyrolysis products from diacetylmorphine and adulterants of street heroin samples. Journal of forensic sciences 2002, 47 (4), 885-888.

39. LASAGNA, L.; De KORNFELD, T. J., ANALGESIC POTENCY OF NORMORPHINE IN PATIENTS WITH POSTOPERATIVE PAIN. Journal of Pharmacology and Experimental Therapeutics 1958, 124 (3), 260-263.

40. Marks, M. G. a. J., The Dependence Phenomenon. MTP Press Limited: 1982. 
41. Paul, D.; Pick, C. G.; Tive, L. A.; Pasternak, G. W., Pharmacological characterization of nalorphine, a kappa 3 analgesic. Journal of Pharmacology and Experimental Therapeutics 1991, 257 (1), 1-7.

42. Rook, E. J.; Van Ree, J. M.; Van Den Brink, W.; Hillebrand, M. J. X.; Huitema, A. D. R.; Hendriks, V. M.; Beijnen, J. H., Pharmacokinetics and Pharmacodynamics of High Doses of Pharmaceutically Prepared Heroin, by Intravenous or by Inhalation Route in Opioid-Dependent Patients. Basic \& Clinical Pharmacology \& Toxicology 2006, 98 (1), 86-96.

43. Kirchheiner, J.; Schmidt, H.; Tzvetkov, M.; Keulen, J. T.; Lötsch, J.; Roots, I.;

Brockmöller, J., Pharmacokinetics of codeine and its metabolite morphine in ultra-rapid metabolizers due to CYP2D6 duplication. The Pharmacogenomics Journal 2006, 7, 257.

44. Iwamoto, K.; Klaassen, C. D., First-pass effect of nalorphine in rats. Journal of Pharmacology and Experimental Therapeutics 1977, 203 (2), 365-376.

45. Garg, A.; Solas, D. W.; Takahashi, L. H.; Cassella, J. V., Forced degradation of fentanyl: Identification and analysis of impurities and degradants. Journal of Pharmaceutical and Biomedical Analysis 2010, 53 (3), 325-334.

46. Manral, L.; Gupta, P.; Suryanarayana, M.; Ganesan, K.; Malhotra, R., Thermal behaviour of fentanyl and its analogues during flash pyrolysis. Journal of thermal analysis and calorimetry 2009, 96 (2), 531-534.

47. Schneider, E.; Brune, K., Opioid activity and distribution of fentanyl metabolites.

Naunyn-Schmiedeberg's archives of pharmacology 1986, 334 (3), 267-274.

48. N-Phenylpropanamide. https://pubchem.ncbi.nlm.nih.gov/compound/N-

Phenylpropanamide\#section=Top.

49. 1,2-Diphenylethane. https://pubchem.ncbi.nlm.nih.gov/compound/7647\#section=Top.

50. Ruan, X.; Chiravuri, S.; Kaye, A. D., Toxicological testing when evaluating fatal cases suspected of acute fentanyl toxicity. Forensic science, medicine, and pathology 2016, 12 (3), 363-365. 


\section{Chapter 4: Detection of Confirmed Thermal Degradation Products of Ten Synthetic Cannabinoids in Post-Mortem Blood Samples}

This chapter is an adaption of a submission to the Journal of Forensic Toxicology in April 2018. Detection of Confirmed Thermal Degradation Products of Ten Synthetic Cannabinoids in PostMortem Blood Samples. S. Raso and S. Bell.

Permission will be obtained from the co-authors and the Journal Forensic Toxicology according to the journal's license's, copyright and permissions policy. A copy of the permissions agreement will be shown in Appendix A if obtained before submission to the college.

The vast toxic effects of synthetic cannabinoids have proven difficult to counteract and fully understand. Adverse effects outside of the common cannabinoid tetrad have been observed in multiple body systems with various causes of death. A relevant hypothesis to the current study is the overlap of symptoms with serotonin syndrome. Current research has begun to link the use of cannabinoids to serotonin syndrome by the finding of thermal degradant products that contain similar core structures. This leads to the notion that the cannabinoid pyrolytics may have binding activity at the serotonin receptors, in which causes the toxic side effect of serotonin syndrome. This report aids in further making this hypothesis a logical possibility. It is the first simultaneous monitoring of parent synthetic cannabinoids and possible thermal degradation products in postmortem blood samples. A validated LC/MS/MS method following a liquid-liquid extraction provided the detection of multiple thermal degradant compounds within the blood samples. The establishment of the pyrolytic ingestion into the body indicates that they cannot be dismissed in the evaluation of the toxicity of synthetic cannabinoids. The degradants presence may impact toxicological assays, metabolic profiles as well as toxic secondhand inhalation of bystanders. The results presented here direct the need for research into the activity of such degradants, determination of their metabolic profile, further investigation into the possibility of serotonin 
syndrome, and added testing of postmortem samples for additional pyrolytics as well as metabolites, which have been observed in previous work on drug pyrolysis.

\subsection{Introduction}

The prevalence of synthetic cannabinoids is a public health issue due to the diverse toxic effects produced. It is challenging to effectively interpret the lethality of synthetic cannabinoids, while also responding to usage outbreaks and identifying new generation compounds. Recently in New York City, 33 individuals were intoxicated in a single neighborhood, and 18 had to be hospitalized ${ }^{1}$. Traditional cannabinoid effects are described using the "cannabinoid tetrad": 1) hypothermia, 2) analgesia, 3) catalepsy and 4) locomotor activity suppression, but adverse toxicity beyond this tetrad are seen in multiple body systems with synthetic abuse ${ }^{2-5}$. Acute kidney injury (AKI) is one of the effects observed in the endocrine system, and is often seen in emergency rooms where synthetic cannabinoid use is coupled with alcohol ${ }^{6-9}$. Cardiovascular effects include tachycardia, hypertension, palpitations and even myocardial infarctions ${ }^{10-14}$. Interestingly, even the nervous system is affected as ischemic stroke has been reported on several occasions $^{15-19}$. 


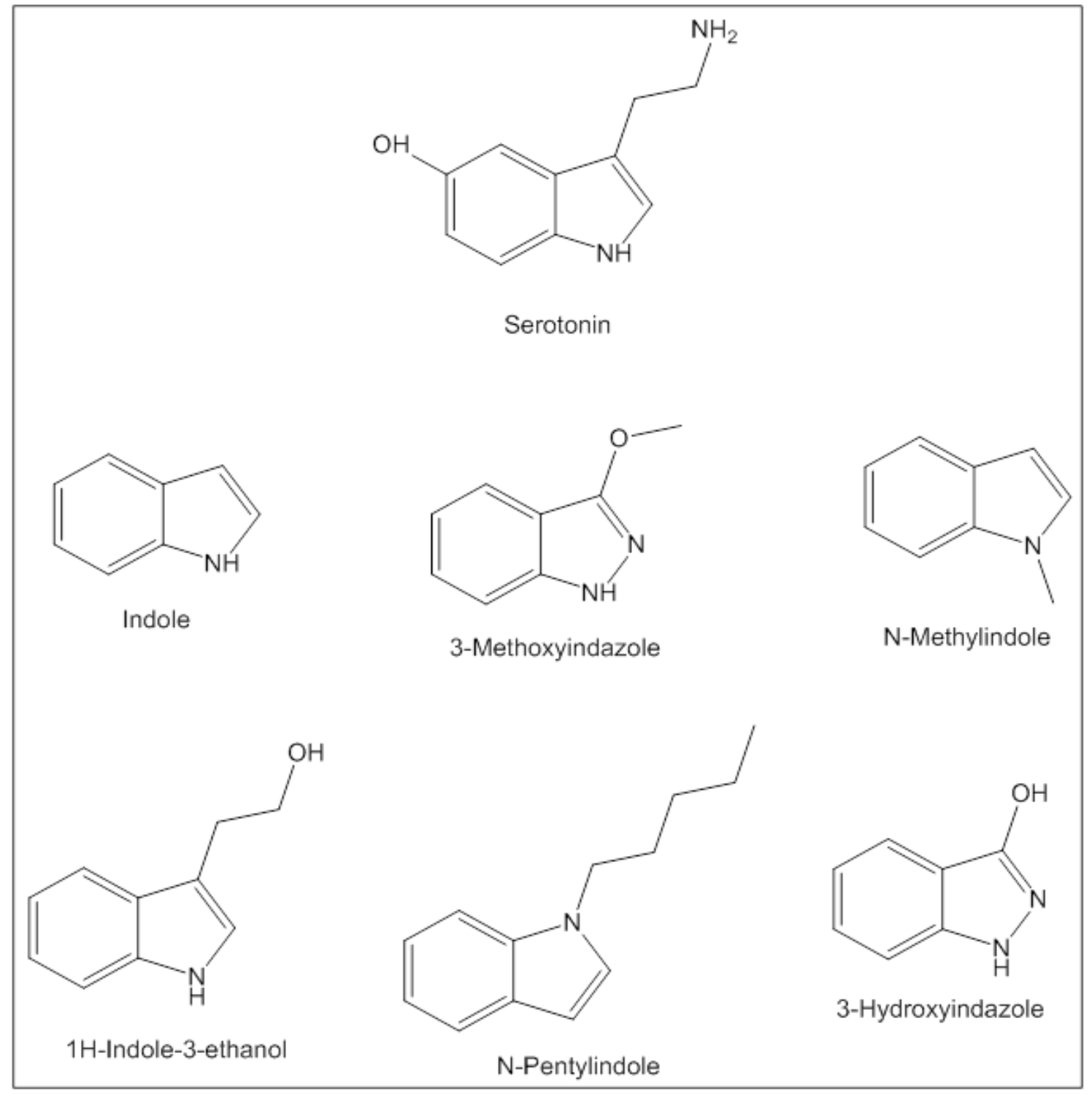

Figure 4.1 - The structure of serotonin and examples of proposed synthetic cannabinoid thermal degradants with similar structure.

In recent years, it has been suggested that some of the observed toxic effects overlap with symptoms of serotonin syndrome ${ }^{20}$. Serotonin syndrome is caused by an over-abundance of serotonin. Numerous symptoms including agitation, confusion, tachycardia and seizures are seen to name a few, but is commonly described as a triad of muscular abnormalities, autonomic hyperactivity and mental-status changes ${ }^{21}$. It is a potentially fatal sickness, and may be a toxic side effect of synthetic cannabinoids ${ }^{22}$. A previous investigation into the thermal degradation of 
cannabinoids may have provided a missing link to connect cannabinoids to serotonin syndrome. Numerous suggested pyrolytic products have contained similar core structures to that of serotonin, which could explain the serotoninic effects, and a few examples are shown in Figure 4.1 ${ }^{23}$. The structural resemblance indicates the possibility of equivalent binding capabilities, thus the abundance of thermal degradants may produce serotonin syndrome like effects.

Based on a global survey in $2017,59.5 \%$ of respondents who ingested cannabinoids did so in herbal form in comparison to powders, resins and oils. Sixty-six percent of those users acknowledged smoking the drug via rolled cigarette ${ }^{24-25}$. Given that smoking primary mode of ingestion, an understanding of the thermal degradation process and products is important in the larger context of toxic effects. The high temperatures reached during pyrolysis induce complex reaction zones where thermal degradation and free radical processes occur. The thermal degradation of a compound produces any number of supplementary compounds to the parent itself. Since pyrolysis is a free radical environment, unique reactions are possible including ring expansions, which are an indication of a pyrolytic process. An evaluation of pyrolysis on illicit drugs over time via a literature review was conducted in 2014 with intriguing results and limitations to the performed studies ${ }^{26}$. Subsequent work by our group has developed a novel smoking simulation apparatus and pyrolysis method to resolve the apparent limitations and applied it to synthetic cannabinoids and classical drugs of abuse $\mathrm{s}^{23}$.

A notable finding of these studies was the formation of metabolites by thermal degradation. Metabolite presence is influential evidence to the use of a particular drug. Current pharmacokinetic models are based off the production of metabolites after the time of ingestion, but this interpretation must be altered if the metabolite is present at the time of exposure within the inhaled vapor. It is also important to take into account the activity of the metabolic product. 
As thermal degradants have been demonstrated to be produced, it is crucial to evaluate the presence of such products within the body to establish their ingestion along with the parent compounds. To date, the two largely detected thermal degradants are the UR-144 and XLR-11 degradants. A recent study has shown that the UR-144 degradant has a fourfold stronger binding affinity at $\mathrm{CB}_{1}$ in comparison to the parent drug ${ }^{27}$. The UR-144 degradant has also been detected in blood as well as its predicted metabolites in urine ${ }^{28}$. The XLR-11 degradant was also found to have its own metabolic profile as the analysis of a known user's urine produced metabolites varying from the parent's profile ${ }^{29}$. However a small sample size, a clear precedent for thermal degradants being ingested is set, as these products would not be produced metabolically after the ingestion of solely the parent drug.

This report is the first to the author's knowledge of a comprehensive study of postmortem blood samples for broad spectrum monitoring of thermal degradation products with known parent drug presence. The obtained blood samples were previously analyzed and found to contain one or more synthetic cannabinoids. Based on the known cannabinoid the detection of predicted pyrolytic products was carried out using a validated and optimized LC/MS/MS method. The results of the study demonstrate that the presence of thermal degradants, including those with ring expansions only produced at high temperatures, and cannot be dismissed. Further research is significant as these products will produce their own unique metabolic and pharmacodynamics profiles.

\subsection{Methods}

\subsubsection{Materials and Reagents}


Each of the solvents used for liquid chromatography analysis including water with $0.1 \%$ formic acid and acetonitrile with $0.1 \%$ formic acid were obtained from Thermo Fisher Scientific (Waltham, MA). The extraction solvents, 0.2M sodium carbonate buffer at $\mathrm{pH}$ 9.4, 99:1 hexaneethyl acetate and 50:50 methanol-acetonitrile with $0.1 \%$ formic acid were prepared using solvents from Thermo Fisher Scientific. Synthetic cannabinoid standards of 5F-ADB, 5F-AMB, 5F-PB-22, AB-CHMINACA, AB-FUBINACA, AB-PINACA, ADB-FUBINACA, FUB-AKB48, MAB-CHMINACA and MMB-FUBINACA were purchased from Cayman Chemical (Ann Arbor, MI). Standards of predicted possible thermal degradation products were obtained as follows: 4-hydroxycinnoline and 3-hydroxyquinoline from Ark Pharm, Inc. (Arlington Heights, IL); indazole-3-carboxaldehyde, indazole, indole, quinoline, N-methylindole, 4-methylquinoline and indole-3-carboxaldehyde from Sigma-Aldrich Chemicals (St. Louis, MO); 1-methylindazole and cinnoline from Alfa Chemistry (Holtsville, NY); pentylindazole, pentylindazole-3carboxaldehyde, pentylindole and pentylindole-3-carboxylic acid were synthesized by the Dr. Gregory Dudley research group at West Virginia University. Human blood for validation studies was obtained from Zen Bio, Inc. (Research Triangle Park, NC). The case sample blood for pyrolytic evaluation was provided by NMS Labs (Willow Grove, PA).

\subsubsection{Sample Preparation}

Stock standard solutions of synthetic cannabinoids were prepared at $1 \mathrm{mg} / \mathrm{ml}$ in either methanol $(\mathrm{MeOH})$, acetonitrile $(\mathrm{ACN})$ or a 50:50 mixture of $\mathrm{MeOH}-\mathrm{ACN}$ dependent on solubility and stored at $-20^{\circ} \mathrm{C}$. Working solutions were prepared by serial dilution at concentrations of 10000, 1000, 100,10,1, 0.1 and $0.01 \mathrm{ng} / \mathrm{ml}$. A $0.2 \mathrm{M}$ sodium carbonate buffer, pH 9.4 was prepared by mixing the contents of a $\mathrm{BupH}^{\mathrm{TM}}$ carbonate-bicarbonate buffer pack to $500 \mathrm{ml}$ of degassed, deionized water. 


\subsubsection{Blood Extraction}

To $1 \mathrm{ml}$ of drug-free blood, $200 \mu \mathrm{l}$ of working synthetic cannabinoid solution was added to obtain the desired concentration. Five hundred microliters of $0.2 \mathrm{M}$ sodium-carbonate buffer, $\mathrm{pH} 9.4$, was added and vortexed for $20 \mathrm{~s}$ followed by the addition of $1.5 \mathrm{ml}$ of 99:1 hexane-ethyl acetate. The samples were mixed for 20 min followed by a 10 min centrifugation. The top organic layer was transferred to a clean vial, evaporated under nitrogen gas at $40^{\circ} \mathrm{C}$, reconstituted in $200 \mu \mathrm{l}$ of mobile phase (50:50 MeOH-ACN with $0.1 \%$ formic acid), and transferred to a labeled autosampler vial. This protocol was adapted from methodology previously reported by Knittel, et $a l^{30}$.

\subsubsection{Instrumental Analysis}

The chromatographic analysis for this study was carried out using a Shimadzu (Houston, TX) prominence high performance liquid chromatograph (HPLC) coupled with an AB SCIEX 3200 QTRAP LC-MS/MS (Framingham, MA) system equipped with a Turbo $\mathrm{V}^{\mathrm{TM}}$ source. The LC was comprised of a DGU-20A3R degasser, LC-20AD pump, SIL-20AC HT autosampler and CTO-20AC oven. Analyst software 1.6.3 was used for data acquisition and analysis.

For chromatic separation, a Phenomenex Kinetex $\mathrm{C}_{18}$ analytical column $(30 \times 2.1 \mathrm{~mm}$ ID $\mathrm{x} 2.6 \mu \mathrm{m}$ ) was utilized (Torrance, CA). The oven compartment was held at $40^{\circ} \mathrm{C}$, and the injection volume was $5 \mu \mathrm{l}$. A gradient elution program was developed with water with $0.1 \%$ formic acid (mobile phase A) and $\mathrm{ACN}$ with $0.1 \%$ formic acid (mobile phase $\mathrm{B}$ ) with a flow rate of $0.5 \mathrm{ml} / \mathrm{min}$. The conditions of the gradient were: starting conditions $20 \% \mathrm{~B}$ and held for 0.3 min, increased to $40 \% \mathrm{~B}$ at $2.3 \mathrm{~min}$, ramped to $50 \% \mathrm{~B}$ at $12.3 \mathrm{~min}$, ramped to $70 \% \mathrm{~B}$ at $14.3 \mathrm{~min}$, returned to $20 \% \mathrm{~B}$ at $15.3 \mathrm{~min}$ and held for $0.6 \mathrm{~min}$ for a total run time of $16.0 \mathrm{~min}$. Baseline 
resolution was not fully achieved, but the target analytes had different transition, which allowed for accurate compound determination.

The mass spectrometer was operated in positive mode with electrospray ionization (ESI) and MRM acquisition. The source dependent parameters during analysis were: GS1 gas was set at 10 psi, GS2 at 0 psi, CUR (curtain) at 10 psi, CAD (collision cell) at medium, IS (ion spray voltage) at 5,500 $\mathrm{V}$ and no heat was applied to the source. Two MRM transitions were monitored for each optimized compound. The optimized compound dependent parameters were determined by direct-infusion analysis with a flow of $10.0 \mathrm{ml} / \mathrm{min}$. The parameters that were optimized included declustering potential (DP), entrance potential (EP), collision cell entrance potential (CEP), collision energy (CE) and collision cell exit potential (CXP). The MRM transitions and optimized parameters for each pertinent compound are listed in the supplementary material; Appendix C. Additional compounds were optimized and monitored as possible pyrolytics, but were omitted from Table $\mathbf{C 1}$ due to not being observed in the blood sample analysis. Additionally, a standard of 4-methylcinnoline was unavailable, so the transition masses are predicted based on the obtained product ions of cinnoline, and the compound dependent parameters were kept the same as cinnoline due to the minor difference of a methyl group in structure.

\subsubsection{Method Validation}

The goal of this study is to observe thermal degradation products in a qualitative manner, however, a number of parameters were validated for the adapted methodology based on a previous report and SWGTOX guidelines including linearity, limit of detection (LOD), limit of quantitation (LOQ), within-day and between-day precision, accuracy, extraction recovery and matrix effects. The linearity was determined by analyzing a multiple concentration calibration 
curve over 10 consecutive days. The linear relationship of the minimum six point curve was evaluated by calculating the regression via the least squares method. The determined $\mathrm{R}^{2}$ values were required to be 0.990 or better. The LOD and LOQ were determined based on back calculating each sample concentration with the generated curve and comparing it to the theoretical concentration. A level of acceptance was set at $\pm 20 \%$. The LOD was defined as the lowest analyzed concentration at which the relative retention time was within $3 \%$ and produced consistent signal intensity, while the LOQ was defined as the lowest concentration that met the set $\pm 20 \%$ criteria.

The instrument precision and accuracy was evaluated at low, middle and high concentrations within the linearity range of 10, 100 and 1,000 ng/ml respectively. The within-day precision was determined by analyzing 5 aliquots of a single extraction. The between-day was evaluated over a 10 day period of a single aliquot. The precision acceptance value was set at a coefficient of variance (CV) or $\leq 20 \%$. As for the accuracy, it was defined as the percent difference of the average calculated concentration and the theoretical fortified concentration. For acceptance, the percent difference was required to be $\leq 20 \%$.

Extraction recovery and matrix effects were evaluated at both a low and high concentration within the linearity range of 50 and $500 \mathrm{ng} / \mathrm{ml}$ respectively, in triplicate. For each examined compound concentration pair, three samples were generated. Sample 1 consisted of the analyte of interest in the extraction solvent. Sample 2 was a pre-extraction sample produced by fortifying reference standard into blank blood and then extracting as previously described. Sample 3 was the post-extraction sample made by adding reference standard after extraction but before the evaporation process. Using these samples, the extraction recovery was determined by the sample 2 response divided by the sample 3 response. The matrix effect was defined as the 
sample 3 response divided by the sample 1 response. A value of over $100 \%$ indicates ion enhancement, while values under $100 \%$ indicate ion suppression.

\subsubsection{Blood Analysis}

Case blood samples were obtained along with the identification of the parent synthetic cannabinoids that were detected during analysis. These identifications are outlined in Table 4.1, and the structures are shown in Figure 4.2. Each compound has an equivalent indazole core structure except 5F-PB-22, which is indole based. Such information was used to predict the thermal degradation products to monitor for in this project, and it is important to note that due to similar structural make-up, they could produce common products. The volume of blood per sample varied from approximately 1-5 ml, and each extraction analysis was carried out on $1 \mathrm{ml}$ portions via the methodology previously described. Where applicable each sample was extracted and analyzed in triplicate, however a few samples were only able to be analyzed once. 
Table 4.1 - Previously detected synthetic cannabinoids within the case blood samples obtained and reported from NMS Labs

\begin{tabular}{|c|c|c|}
\hline Description & Sample Number & Compound \\
\hline \multirow{8}{*}{ Single Drug } & 1 & AB-PINACA \\
\hline & 2,21 & AB-FUBINACA \\
\hline & 3,17 & MAB-CHMINACA \\
\hline & $4,18,22,23$ & ADB-FUBINACA \\
\hline & $5,6,7,9,10,13,16,19$ & 5F-ADB \\
\hline & 8 & MMB-FUBINACA \\
\hline & 14 & 5F-AMB \\
\hline & Sample Number & Compounds \\
\hline \multirow{4}{*}{ Mixtures } & 11 & $\begin{array}{c}\text { 5F-ADB } \\
\text { MMB-FUBINACA }\end{array}$ \\
\hline & 12 & $\begin{array}{c}\text { FUB-AKB-48 } \\
\text { AB-CHMINACA } \\
\text { MAB-CHMINACA }\end{array}$ \\
\hline & 15 & $\begin{array}{c}\text { 5F-PB22 } \\
\text { FUB-AKB-48 } \\
\text { AB-CHMINACA } \\
\text { MAB-CHMINACA }\end{array}$ \\
\hline & 20 & $\begin{array}{c}\text { 5F-ADB } \\
\text { ADB-FUBINACA }\end{array}$ \\
\hline
\end{tabular}




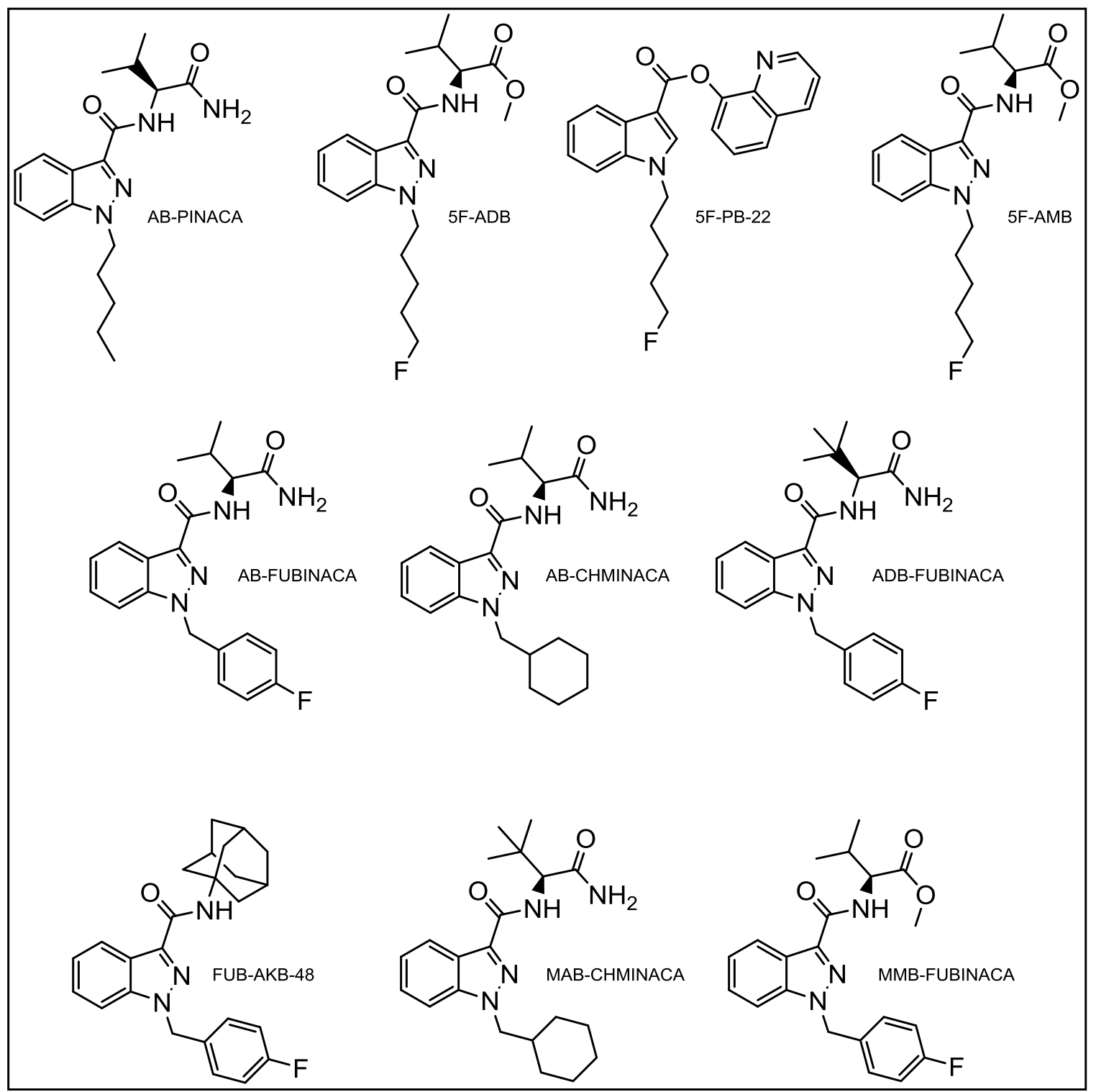

Figure 4.2 - The structure of each parent synthetic compound contained in the 23 case blood samples

\subsection{Results}

\subsubsection{Method Validation}

Linearity was assessed using eight concentrations ranging from $0.01-1,000 \mathrm{ng} / \mathrm{ml}$ comprised of $0.01,0.1,1,10,25,50,100$ and $1,000 \mathrm{ng} / \mathrm{ml}$ standard concentrations. The 8 concentrations were necessary as the low end concentrations were not consistently observed and at least six points were desired for the calibration curves. The linearity was found to be 
acceptable across the analyzed concentrations for each compound based on the produced calibration curve $\mathrm{R}^{2}$ values. The LOD was commonly observed to be approximately $1 \mathrm{ng} / \mathrm{ml}$ but as low as $0.01 \mathrm{ng} / \mathrm{ml}$. As for the LOQ's, they ranged from $1-10 \mathrm{ng} / \mathrm{ml}$. The experimentally obtained values for each compound are summarized in Table C2.

The within-day and between day precision and accuracy were evaluated at a low, mid and high concentration across the linearity range, and chosen levels were 10, 100 and 1,000 ng/ml. All within-day and between-day precisions were acceptable with the exception of the 1,000 $\mathrm{ng} / \mathrm{ml}$ samples of AB-PINACA being scarcely unacceptable with CV values for between-day and within-day of 20.5 and $20.7 \%$ respectively, and the $1,000 \mathrm{ng} / \mathrm{ml}$ between-day sample for FUBAKB-48 and $100 \mathrm{ng} / \mathrm{ml}$ within-day sample of 5F-ADB at 20.9 and 20.3 respectively. The between-day accuracies were accepted with the exception of MMB-FUBINACA at $100 \mathrm{ng} / \mathrm{ml}$ with a percent difference of $21.9 \%$. The between-day accuracies all fell under $20 \%$. The data of all calculated precision and accuracy values are detailed in Table C3.

The extraction efficiency and matrix effects were evaluated at two concentrations, 50 and $500 \mathrm{ng} / \mathrm{ml}$, which were a low and high concentration value within the linear ranges. The corresponding data is within Table C4. The extraction recoveries ranged from $64.7-165.16 \%$. The extraction protocol was taken from a previous report, which had a recovery range of $70-$ $174 \%^{30}$. The recoveries reported here are comparable with a slightly smaller overall range. The matrix effects were determined to range from $41.91-155.71 \%$, which again was comparable to the previous report as their reported range was $27-147 \%^{30}$. The majority of the samples were under 100\%, indicating ion suppression, but when ion enhancement was observed, it was at a much larger rate. Twelve of the 30 total samples, nearly half were within $\pm 20 \%$, showing little matrix effects from the blood. 


\subsubsection{Blood Analysis}

The samples were analyzed up to three times dependent on the amount of blood supplied. Thermal degradation products were detected in each sample except one, which may have been due to the small volume of blood obtained or due to the user not ingesting the drug compound via a smoking process. Six compounds were consistently observed at a signal level of greater than three times the noise level. The results and structures of the observed pyrolytic products are outlined in Table 4.2 and Figure 4.3. Each contained either the core indazole ring or the expanded ring form of indazole, cinnoline, which is a process indicative of pyrolysis. From the samples containing a single drug, 4-hydroxycinnoline, 1H-indazole-3-carboxaldehyde and cinnoline were commonly observed, which is emblematic of each parent drug containing structurally similar cores. The pyrolytic products observed within the mixtures are consistent with expected results based on the analyses on the single drug samples. 
Table 4.2 - Observed thermal degradation products from the case sample blood samples. 1: 4-Hydroxycinnoline; 2: 1H-Indazole-3-carboxaldehyde; 3: Indazole; 4: Cinnoline;

5: N- Methylindazole; 6: 4-Methylcinnoline; 7: N-Pentylindazole. *- Not Confirmed

\begin{tabular}{|c|c|c|c|}
\hline & Compound & TD(s) Observed & Frequency \\
\hline \multirow{12}{*}{$\begin{array}{l}\text { Single } \\
\text { Drug }\end{array}$} & \multirow{4}{*}{$5 \mathrm{~F}-\mathrm{ADB}$} & 1 and 2 & 6 of 8 \\
\hline & & 4 and 5 & 4 of 8 \\
\hline & & $6^{*}$ & 3 of 8 \\
\hline & & 7 & 1 of 8 \\
\hline & 5F-AMB & 1,2 and 4 & 1 of 1 \\
\hline & \multirow{2}{*}{ AB-FUBINACA } & 1 and 2 & 2 of 2 \\
\hline & & 3 and 4 & 1 of 2 \\
\hline & AB-PINACA & 1 and 2 & 1 of 1 \\
\hline & \multirow{2}{*}{ ADB-FUBINACA } & 1 and 2 & 4 of 4 \\
\hline & & 4 & 1 of 4 \\
\hline & MMB-FUBINACA & 1,2 and 7 & 1 of 1 \\
\hline & Compounds & \multicolumn{2}{|c|}{ TD(s) Observed } \\
\hline \multirow{4}{*}{ Mixtures } & $\begin{array}{c}\text { 5F-ADB } \\
\text { MMB-FUBINACA }\end{array}$ & \multicolumn{2}{|l|}{ N/A } \\
\hline & $\begin{array}{c}\text { AB-CHMINACA } \\
\text { FUB-AKB-48 } \\
\text { MAB-CHMINACA }\end{array}$ & \multicolumn{2}{|c|}{1 and 2} \\
\hline & $\begin{array}{c}\text { 5F-PB-22 } \\
\text { AB-CHMINACA } \\
\text { FUB-AKB-48 } \\
\text { MAB-CHMINACA }\end{array}$ & \multicolumn{2}{|c|}{1,2 and $6^{*}$} \\
\hline & $\begin{array}{c}\text { 5F-ADB } \\
\text { ADB-FUBINACA }\end{array}$ & \multicolumn{2}{|c|}{$1,2,4$ and 5} \\
\hline
\end{tabular}




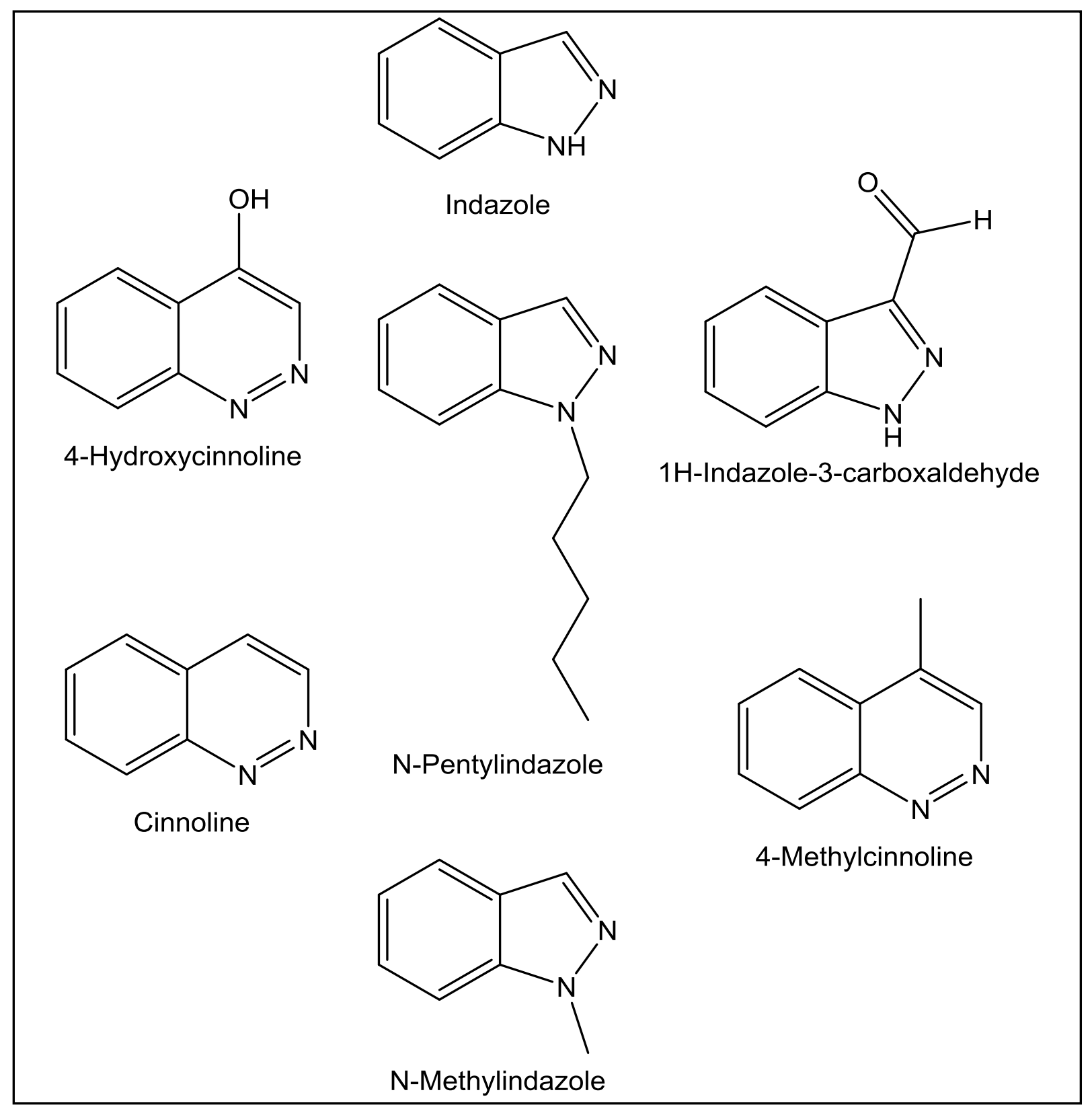

Figure 4.3 - The structure of the 7 consistently observed thermal degradation products 


\subsection{Discussion}

As the results of the current study began to evolve, a review of unwanted factors was conducted. The assurance of the pyrolytics being present within the blood itself and not produced through analysis, specifically the electrospray ion source (ESI), was confirmed as the degradants eluted at different times than the parent compounds. If they were produced within the ESI, the retention times would be equivalent as the ESI is applied after the chromatography. The collection time of the blood relative to the ingestion time is unknown, so it is understood that additional pyrolytics may have been present and already undergone metabolism. Urine analysis would need to be carried out to fully understand the spectrum of degradants and their metabolites that may be present for toxicological assays, along with a time course for such breakdown.

Nineteen of the 23 blood samples contained a single drug compound, and in comparing the results within samples containing the same drug, a few patterns are apparent. Each of the 6 parent cannabinoids produced both 4-hydroxycinnoline and 1H-indazole-3-carboxaldehyde. Cinnoline was present in samples of 4 of the 6 parent cannabinoids, which is the other predominantly observed pyrolytic. The remaining 4 degradants seen were more sporadic and typically in a relatively lower abundance. The greater abundance and more consistent production of degradants 1,2 and 4 is to be expected based on the predicted breakdown of the parent cannabinoids. The amine bond located within the indazole ring and the amide group would be expected to cleave as carbon-nitrogen bonds are relatively weak. This process would produce compound 2, and then a ring expansion process of the indazole ring allows for the production of compound 1 . Lastly, the dihydroxylation of compound 1 would produce compound 4.

The data analysis of the single drug samples also provided some anomalies. There were 8 samples containing solely 5F-ADB, but two samples did not appear to produce all of the same 
degradants as the other 6. Samples 13 and 16 produced only compound 6, 4-methylcinnoline. It is first important to note that both of these samples had a small volume of blood and were only able to be analyzed once. The presence of compound 6 in general was interesting as it was never observed in samples where compound 4, cinnoline, was present. These degradants differ by only a methyl group, and as previously described, the MRM of 4-methylcinnoline was predicted based off of the cinnoline optimized parameters. Further confirmation of 4-methylcinnoline is necessary to definitively determine if in fact both compounds are present or just one. As for ABPINACA, 5F-AMB and MMB-FUBINACA, there was only one single drug sample, thus further replicates of these is desired to support the observed products, but the observed thermal degradants is understandable based on the resemblance between them and the other parent drugs analyzed.

The thermal degradants within the mixtures appeared to coincide with those observed within the single drug samples, with the exception of sample 11, which produced no results. Sample 11 had less than $1 \mathrm{~mL}$ of blood, which may have had an effect on the lack of pyrolytic product detection, and additionally, the user may have not smoked the drug as the route of ingestion. Sample 12 contained AB-CHMINACA, FUB-AKB-48 and MAB-CHMINACA, which were not amongst the single drug samples. Compounds 1 and 2 were detected and justified as these cannabinoids differ in structure of substituent groups, not the core structure producing such degradants. However, it cannot be confirmed which compound may have produced the degradants or if each did individually. Sample 15 contained the most diversity of cannabinoids, 4 different compounds. As in sample 12, the degradants could be present due to any of the parent cannabinoids incorporated in the sample except for 5F-PB-22. This is the only instance of 5FPB-22, but at this time it cannot be stated that it provided any of the current pyrolytics as it 
contains an indole base versus the indazole base presently detected. Further analysis into 5F-PB22 and other indole based cannabinoids may yield thermal degradants not observed here. The final mixture, sample 20, contained 5F-ADB and ADB-FUBINACA, which were the two cannabinoids analyzed as single drugs at the highest rate. Degradant compounds 1, 2, 4 and 5 were detected. Compound 5 had not been detected in the ADB-FUBINACA samples, and is perceived to be produced by 5F-ADB, while the other 3 could be present due to either compound or both.

It is challenging to unambiguously define what makes two compounds "similar" to one another, but for the purposes of this discussion, the detected pyrolytics are deemed "similar" to serotonin based on their structural base. Serotonin consists of an indole ring structure with an alkyl amine chain and a hydroxyl group. The seven thermal degradants described here contain either an indazole or cinnoline ring base. These differ from the serotonin indole base by only a substitution of nitrogen for carbon in indazole or a single carbon increase in ring size. It is plausible that these base ring structures would interact with the same receptor binding sites as serotonin. Their presence could then be a factor in the symptoms that overlap with serotonin syndrome.

\subsection{Conclusion}

The detection of various thermal degradant products in the current study demonstrates the necessity for further research into their activity, metabolism and possible toxicity. They cannot be dismissed as a frivolous exploration. This report coupled with previous reports clearly establishes not only the production of pyrolytic products, but their ingestion by a user while smoking such cannabinoids. The findings presented here also take the next step in connecting cannabinoids to the possible toxic side effect of serotonin syndrome. It is now established that 
numerous degradants with similar core structures to serotonin are not only produced, but also are ingested by the user. As their presence in the vapor is known, innocent bystanders may also ingest the pyrolytic products. Further research endeavors in this area may aid in answering some relevant issues created with synthetic cannabinoids.

\subsection{Funding}

This work was supported by the National Institute of Justice [2015-R2-CX-0032].

\subsection{Acknowledgements}

The author's would like to thank NMS Labs for providing the case blood samples used for analysis in this study. Also, a thank you goes out to the Dr. Greg Dudley research lab at WVU for synthesizing a number of standard compounds for the project.

\subsection{References}

1. Adams, A. J.; Banister, S. D.; Irizarry, L.; Trecki, J.; Schwartz, M.; Gerona, R., "Zombie" Outbreak Caused by the Synthetic Cannabinoid AMB-FUBINACA in New York. $N$ Engl J Med 2017, 376 (3), 235-242.

2. Castellanos, D.; Singh, S.; Thornton, G.; Avila, M.; Moreno, A., Synthetic cannabinoid use: a case series of adolescents. The Journal of adolescent health : official publication of the Society for Adolescent Medicine 2011, 49 (4), 347-9.

3. Seely, K. A.; Lapoint, J.; Moran, J. H.; Fattore, L., Spice drugs are more than harmless herbal blends: a review of the pharmacology and toxicology of synthetic cannabinoids. Progress in neuro-psychopharmacology \& biological psychiatry 2012, 39 (2), 234-43.

4. $\quad$ Marshell, R.; Kearney-Ramos, T.; Brents, L. K.; Hyatt, W. S.; Tai, S.; Prather, P. L.; Fantegrossi, W. E., In vivo effects of synthetic cannabinoids JWH-018 and JWH-073 and phytocannabinoid Delta(9)-THC in mice: Inhalation versus intraperitoneal injection. Pharmacol. Biochem. Behav. 2014, 124, 40-47.

5. Every-Palmer, S., Warning: Legal Synthetic Cannabinoid-Receptor Agonists such as JWH-018 may Precipitate Psychosis in Vulnerable Individuals. Addiction 2010, 105, 1859-1860.

6. Buser, G. L.; Gerona, R. R.; Horowitz, B. Z.; Vian, K. P.; Troxell, M. L.; Hendrickson, R. G.; Houghton, D. C.; Rozansky, D.; Su, S. W.; Leman, R. F., Acute kidney injury associated with smoking synthetic cannabinoid. Clinical toxicology 2014, 52 (7), 664-73.

7. Bhanushali, G. K.; Jain, G.; Fatima, H.; Leisch, L. J.; Thornley-Brown, D., AKI associated with synthetic cannabinoids: a case series. Clin J Am Soc Nephrol 2013, 8 (4), 523-6.

8. Pendergraft, W. F., 3rd; Herlitz, L. C.; Thornley-Brown, D.; Rosner, M.; Niles, J. L., Nephrotoxic effects of common and emerging drugs of abuse. Clin J Am Soc Nephrol 2014, 9 (11), 1996-2005. 
9. Thornton, S. L.; Wood, C.; Friesen, M. W.; Gerona, R. R., Synthetic cannabinoid use associated with acute kidney injury. Clinical toxicology 2013, 51 (3), 189-90.

10. Mir, A.; Obafemi, A.; Young, A.; Kane, C., Myocardial infarction associated with use of the synthetic cannabinoid K2. Pediatrics 2011, 128 (6), e1622-7.

11. Hermanns-Clausen, M.; Kneisel, S.; Szabo, B.; Auwarter, V., Acute toxicity due to the confirmed consumption of synthetic cannabinoids: clinical and laboratory findings. Addiction 2013, 108 (3), 534-44.

12. Schwartz, M. D.; Trecki, J.; Edison, L. A.; Steck, A. R.; Arnold, J. K.; Gerona, R. R., A Common Source Outbreak of Severe Delirium Associated with Exposure to the Novel Synthetic Cannabinoid ADB-PINACA. J Emerg Med 2015, 48 (5), 573-80.

13. Young, A. C.; Schwarz, E.; Medina, G.; Obafemi, A.; Feng, S. Y.; Kane, C.;

Kleinschmidt, K., Cardiotoxicity associated with the synthetic cannabinoid, K9, with laboratory confirmation. Am J Emerg Med 2012, 30 (7), 1320 e5-7.

14. Schneir, A. B.; Cullen, J.; Ly, B. T., "Spice" girls: synthetic cannabinoid intoxication. J Emerg Med 2011, 40 (3), 296-9.

15. Degirmenci, Y.; Kececi, H.; Olmez, N., A case of ischemic stroke after bonzai: syntetic cannabinoid from Turkey. Neurological Sciences 2016, 2 (37), 299-300.

16. Freeman, M. J.; Rose, D. Z.; Myers, M. A.; Gooch, C. L.; Bozeman, A. C.; Burgin, W. S., Ischemic stroke after use of the synthetic marijuana "spice". Neurology 2013, 81 (24), 20902093.

17. Inal, T.; Köse, A.; Köksal, Ö.; Armagan, E.; Aydın, S. A.; Ozdemir, F., Acute temporal lobe infarction in a young patient associated with marijuana abuse: an unusual cause of stroke. World journal of emergency medicine 2014, 5 (1), 72.

18. Bernson-Leung, M. E.; Leung, L. Y.; Kumar, S., Synthetic cannabis and acute ischemic stroke. J Stroke Cerebrovasc Dis 2014, 23 (5), 1239-41.

19. Dogan, B.; Dogru, H.; Gungor, L.; Balci, K., Stroke due to Bonzai use: two patients. World journal of emergency medicine 2016, 7 (4), 310.

20. Louh, I. K.; Freeman, W. D., A 'spicy' encephalopathy: synthetic cannabinoids as cause of encephalopathy and seizure. Crit Care 2014, 18 (5), 553.

21. Boyer, E. W.; Shannon, M., The serotonin syndrome. New England Journal of Medicine 2005, 352 (11), 1112-1120.

22. Sampson, E.; Warner, J. P., Serotonin syndrome: potentially fatal but difficult to recognize. The British Journal of General Practice 1999, 49 (448), 867.

23. Raso, S.; Bell, S., Qualitative Analysis and Detection of the Pyrolytic Products of JWH018 and 11 Additional Synthetic Cannabinoids in the Presence of Common Herbal Smoking Substrates. Journal of analytical toxicology 2017, 41 (6), 551-558.

24. Survey, G. D., Methods in which synthetic cannabinoids were used globally as of 2016. Statista, Ed. 2016.

25. Survey, G. D., Percentage of synthetic cannabinoid users that tried various preparations of this drug from 2015-2017. Statista, Ed. 2017.

26. Bell, S.; Nida, C., Pyrolysis of drugs of abuse: a comprehensive review. Drug testing and analysis 2015, 7 (6), 445-56.

27. Kaizaki-Mitsumoto, A.; Hataoka, K.; Funada, M.; Odanaka, Y.; Kumamoto, H.;

Numazawa, S., Pyrolysis of UR-144, a synthetic cannabinoid, augments an affinity to human $\mathrm{CB} 1$ receptor and cannabimimetic effects in mice. The Journal of toxicological sciences $\mathbf{2 0 1 7}$, 42 (3), 335-341. 
28. Adamowicz, P.; Zuba, D.; Sekula, K., Analysis of UR-144 and its pyrolysis product in blood and their metabolites in urine. Forensic science international 2013, 233 (1-3), 320-7.

29. Kanamori, T.; Kanda, K.; Yamamuro, T.; Kuwayama, K.; Tsujikawa, K.; Iwata, Y. T.; Inoue, H., Detection of main metabolites of XLR-11 and its thermal degradation product in human hepatoma HepaRG cells and human urine. Drug testing and analysis 2015.

30. Knittel, J. L.; Holler, J. M.; Chmiel, J. D.; Vorce, S. P.; Magluilo, J., Jr.; Levine, B.; Ramos, G.; Bosy, T. Z., Analysis of Parent Synthetic Cannabinoids in Blood and Urinary Metabolites by Liquid Chromatography Tandem Mass Spectrometry. Journal of analytical toxicology 2016, 40 (3), 173-86. 


\section{Chapter 5: Conclusions and Future Directions}

\subsection{Impact of Conclusions}

This study has successfully identified numerous thermal degradation products for a representative group of synthetic cannabinoids, established a predictive model for forecasting thermal degradation products of cannabinoids not yet analyzed, validated an LC/MS/MS method for a collection of synthetic cannabinoids and detected a number of thermal degradants within post mortem blood samples. The current research has reiterated the lack of knowledge in the field of drug pyrolysis, and demonstrates a strong proof of concept insight to open discussion into the effect of thermal degradation products on the toxicological effects of synthetic cannabinoids.

The first phase of this project developed a novel approach to mimicking a smoking process while utilizing an exhaustive sample collection method for the pyrolysis of synthetic cannabinoids. The results of the pyrolysis studies unveiled that a single synthetic cannabinoid may produce up to $10-15$ thermal degradation products. The observed predicted products contained compounds that share core structures to previously known toxic compounds. The pyrolytic products may produce similar toxicity to users upon inhalation as well as bystanders whom may inhale the produced vapors.

Following the demonstration of thermal degradation product formation, to provide a supported possibility of such products being toxic, they must first be proven to be ingested. True case sample post mortem blood was obtained to evaluate the presence of thermal degradation products. The set of synthetic cannabinoids present within the blood samples were previously identified, and a new LC/MS/MS method was validated for the aforementioned cannabinoids 
after optimization of all pertinent compounds. Prior to the current study, only two thermal degradants have been detected in toxicological specimen. This study expands the thermal degradants detected in post mortem samples, and supports the need for continued research investigations into the metabolism, binding activity and toxicity of set products.

Fields of forensic science including drug analysis, toxicology assays and medicolegal investigations can profit from the results of this study. Drug paraphernalia can be analyzed for degradant products left behind to establish a basis for the investigation as certain markers may indicate the use of particular drug compounds. The thermal degradation products are also markers in toxicology specimen to indicate that the mode of ingestion was smoking or inhalation. Lastly, as effects of the thermal degradants are defined, the gained knowledge will be impactful on medicolegal assessments.

\subsection{Future Directions}

As this was the first broad monitoring of pyrolytic products in post mortem blood, additional studies for the identification of more pyrolytics should be conducted. As more pyrolytic products are identified, the compounds can be added to monitoring assays and evaluated for their presence as well. Post mortem samples must be continued to be evaluated for not only the parent cannabinoids, but their pyrolytic products too. After the determination of other pyrolytics utilizing the established methodology, a quantitation step must be developed.

As quantitation of pyrolytic products within toxicology specimen is performed, a number of important pieces of information may be obtained. First and foremost, it will provide insight into the types of pyrolytics that are most commonly ingested and need to be further researched. Once the methodology is set, controlled inhalation studies may be conducted to determine a percent breakdown of the parent cannabinoid as well as rates of ingestion. This type of research 
would need to be carried out using mice or rats, as it would be unethical to have human first start smoking such dangerous drug compounds. The rates of ingestion could be adapted for an estimated value in humans, and provide a number of products that should be evaluated for their effects post ingestion.

After the thermal degradants are ingested they will have their own metabolic profile. Metabolism studies may be carried out on the pyrolytic products that displayed the most promise in the ingestion rates studies. The unique metabolites identified from the pyrolytics will offer markers for the route of ingestion in urine samples to couple with the parent pyrolytic products in blood. The parent cannabinoid compounds are not always present by the time specimen are collected, so the additional target analytes provided by the pyrolytic products and their metabolites vastly increase the chances of the detection of a marker indicating the use of synthetic cannabinoids.

Once an encompassing set of target analytes is completed, it would be necessary to evaluate their activity within the body. The products may still hold affinity to the cannabinoid receptors, or as previously mentioned, a number of pyrolytics may have an affinity for serotonin receptors. The overall activity of each product as well as their metabolites would need assessed to completely understand how a user may be affected by smoking synthetic cannabinoids. Not only is the pharmacology of the products crucial, but also their possible toxicity. Toxicity studies should be performed to evaluate if their presence may be one of the causes of the additional toxicity observed with synthetic cannabinoids outside of the common tetrad. There is valuable information to be gained from further investigation into the pyrolysis of synthetic cannabinoids and their thermal degradation products. They cannot simply be dismissed as a non-factor at this point with fully evaluating all of these possibilities in future research endeavors. 


\section{Appendix A}

\section{A copy of the permissions agreement from the Journal of Analytical Toxicology \\ OXFORD UNIVERSITY PRESS LICENSE \\ TERMS AND CONDITIONS}

Mar 06, 2018

This Agreement between Stephen Raso ("You") and Oxford University Press ("Oxford

University Press") consists of your license details and the terms and conditions provided by Oxford University Press and Copyright Clearance Center.

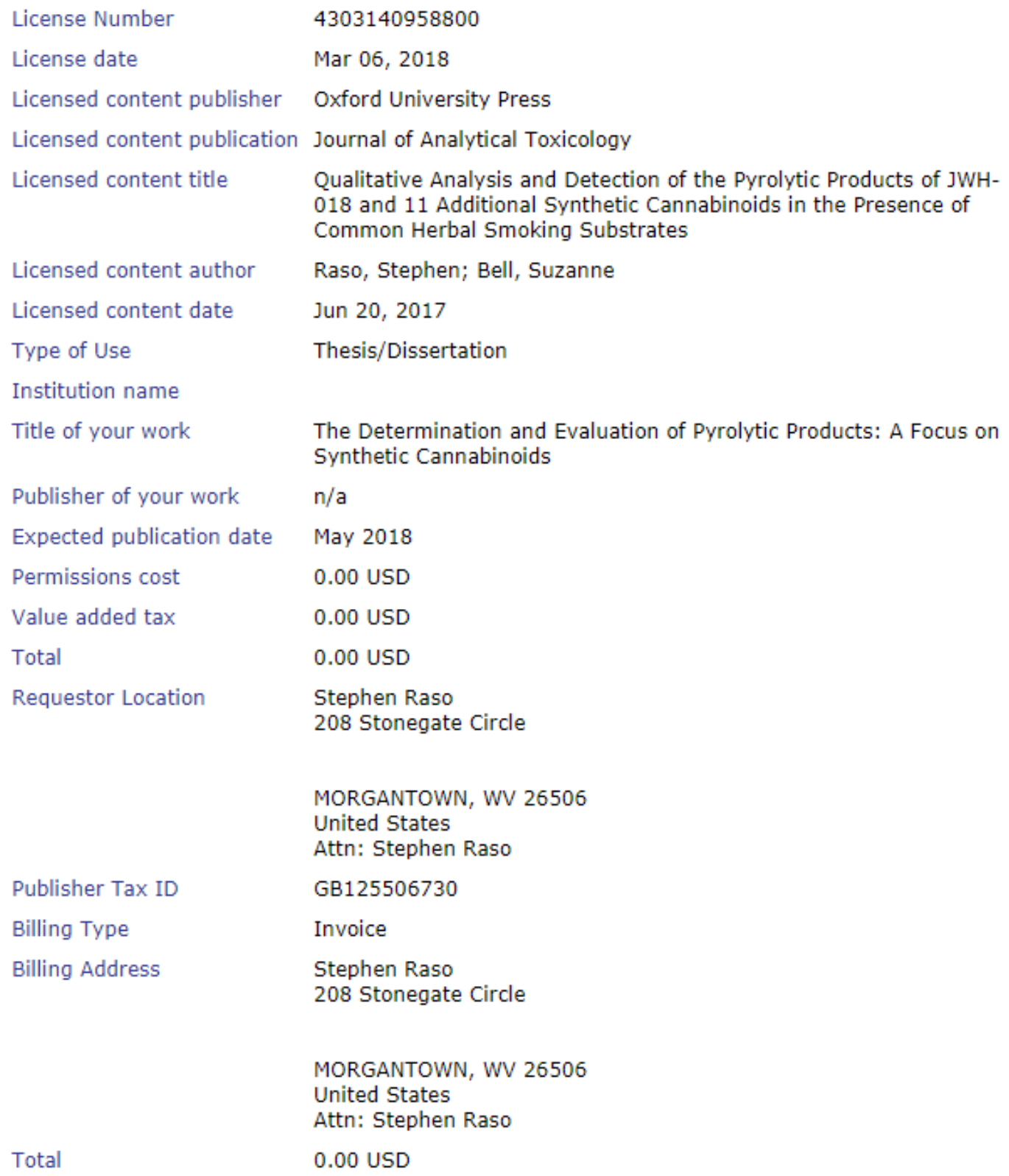




\section{STANDARD TERMS AND CONDITIONS FOR REPRODUCTION OF MATERIAL FROM AN OXFORD UNIVERSITY PRESS JOURNAL}

1. Use of the material is restricted to the type of use specified in your order details.

2. This permission covers the use of the material in the English language in the following territory: world. If you have requested additional permission to translate this material, the terms and conditions of this reuse will be set out in clause 12 .

3. This permission is limited to the particular use authorized in (1) above and does not allow you to sanction its use elsewhere in any other format other than specified above, nor does it apply to quotations, images, artistic works etc that have been reproduced from other sources which may be part of the material to be used.

4. No alteration, omission or addition is made to the material without our written consent. Permission must be re-cleared with Oxford University Press if/when you decide to reprint.

5. The following credit line appears wherever the material is used: author, title, journal, year, volume, issue number, pagination, by permission of Oxford University Press or the sponsoring society if the journal is a society journal. Where a journal is being published on behalf of a learned society, the details of that society must be included in the credit line.

6. For the reproduction of a full article from an Oxford University Press journal for whatever purpose, the corresponding author of the material concerned should be informed of the proposed use. Contact details for the corresponding authors of all Oxford University Press journal contact can be found alongside either the abstract or full text of the article concerned, accessible from www.oxfordjournals.org Should there be a problem clearing these rights, please contact journals.permissions@oup.com

7. If the credit line or acknowledgement in our publication indicates that any of the figures, images or photos was reproduced, drawn or modified from an earlier source it will be necessary for you to clear this permission with the original publisher as well. If this permission has not been obtained, please note that this material cannot be included in your publication/photocopies.

8. While you may exercise the rights licensed immediately upon issuance of the license at the end of the licensing process for the transaction, provided that you have disclosed complete and accurate details of your proposed use, no license is finally effective unless and until full payment is received from you (either by Oxford University Press or by Copyright Clearance Center (CCC)) as provided in CCC's Billing and Payment terms and conditions. If full payment is not received on a timely basis, then any license preliminarily granted shall be deemed automatically revoked and shall be void as if never granted. Further, in the event that you breach any of these terms and conditions or any of CCC's Billing and Payment terms and conditions, the license is automatically revoked and shall be void as if never granted. Use of materials as described in a revoked license, as well as any use of the materials beyond the scope of an unrevoked license, may constitute copyright infringement and Oxford University Press reserves the right to take any and all action to protect its copyright in the materials.

9. This license is personal to you and may not be sublicensed, assigned or transferred by you to any other person without Oxford University Press's written permission.

10. Oxford University Press reserves all rights not specifically granted in the combination of (i) the license details provided by you and accepted in the course of this licensing transaction, (ii) these terms and conditions and (iii) CCC's Billing and Payment terms and conditions.

11. You hereby indemnify and agree to hold harmless Oxford University Press and CCC, and their respective officers, directors, employs and agents, from and against any and all claims arising out of your use of the licensed material other than as specifically authorized pursuant to this license.

12. Other Terms and Conditions:

v1.4 


\section{Appendix B}

Supplementary Material for Chapter 2: Qualitative Analysis and Detection of the Pyrolytic Products of JWH-018 and 11 Additional Synthetic Cannabinoids in the Presence of Common Herbal Smoking Substrates

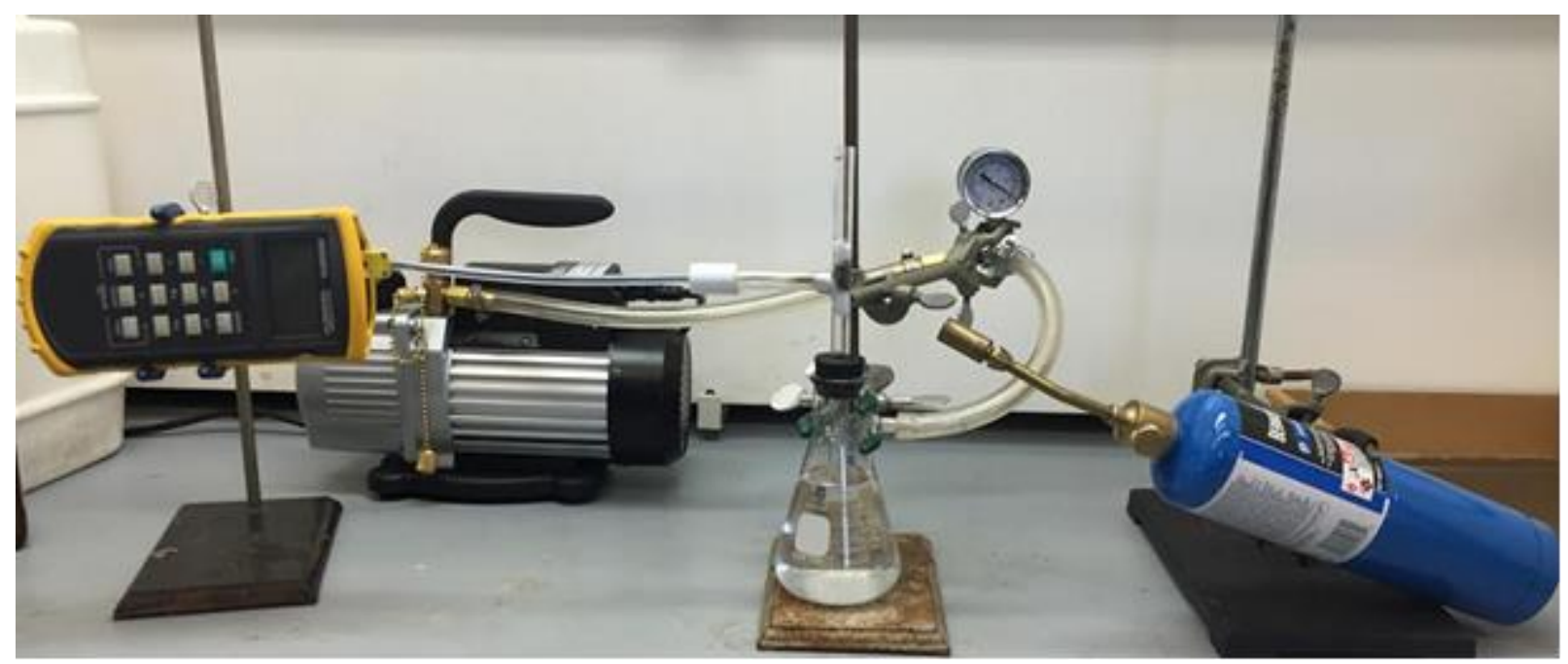

Figure B1 - Photograph of the in-house built apparatus 


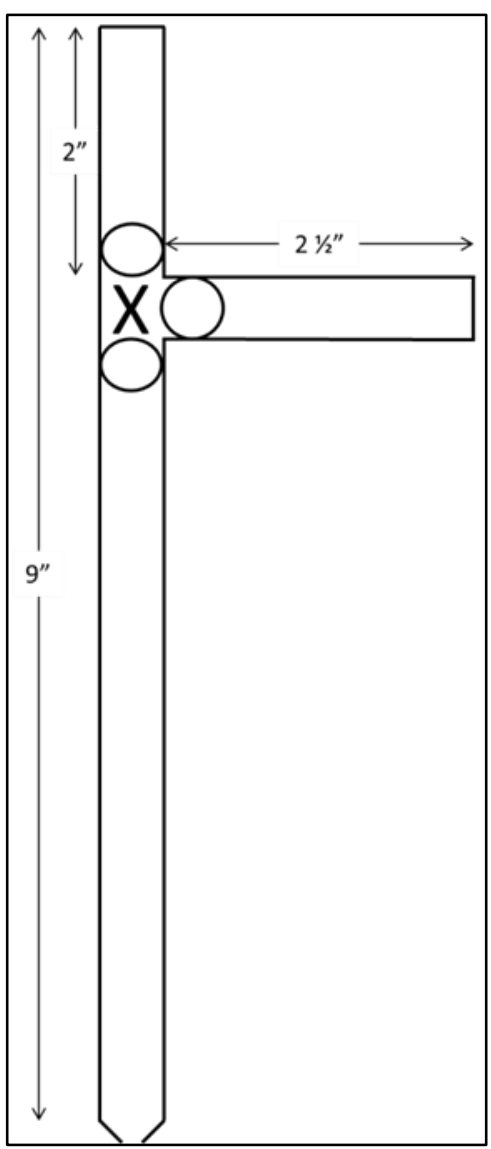

Figure B2 - Schematic of the quartz tubing T-junction where the pyrolysis was contained 


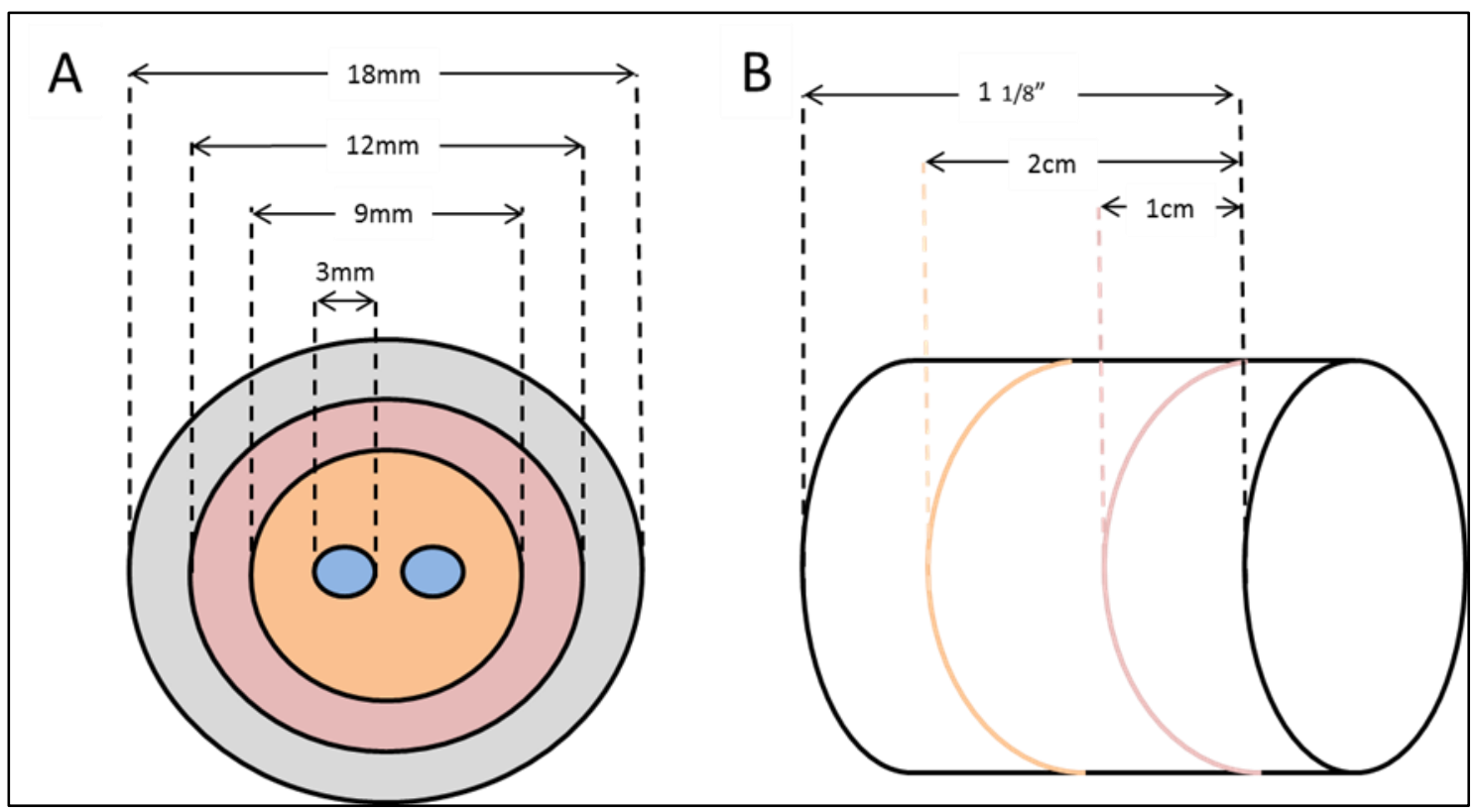

Figure B3 - Schematic of the bored Teflon plug used to hold the pyrolysis apparatus parts and seal system

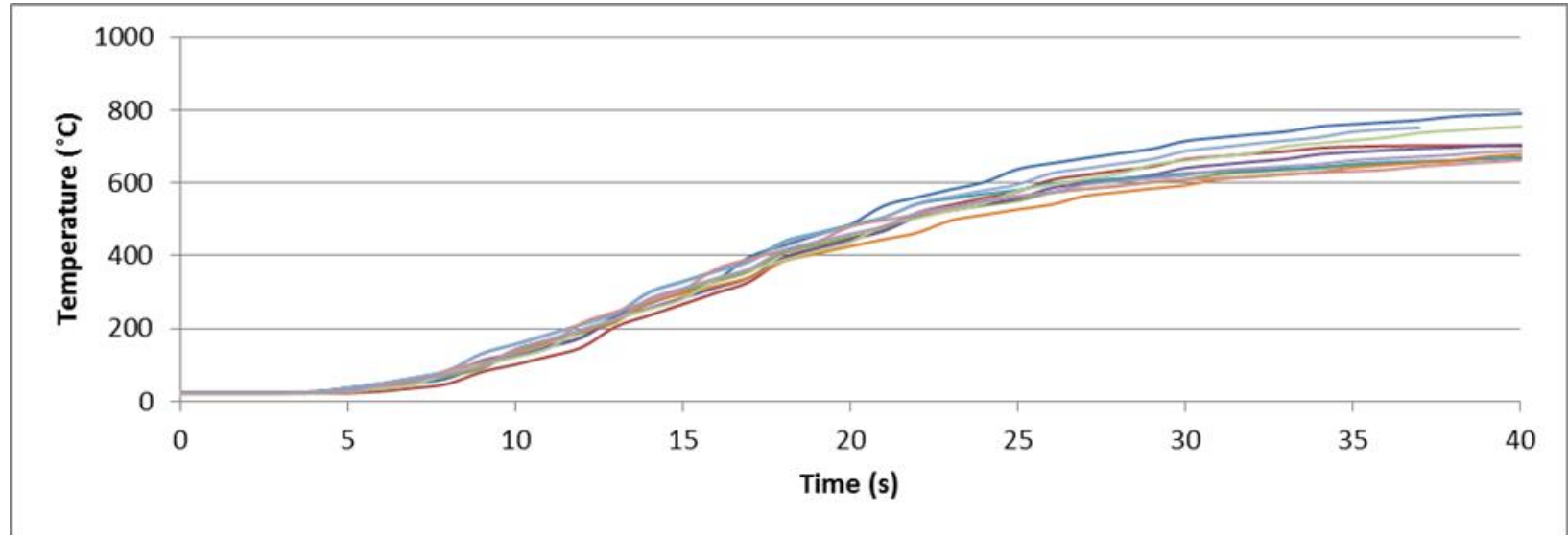

Figure B4 - The heating signature data of the propane torch with no herbal material present 


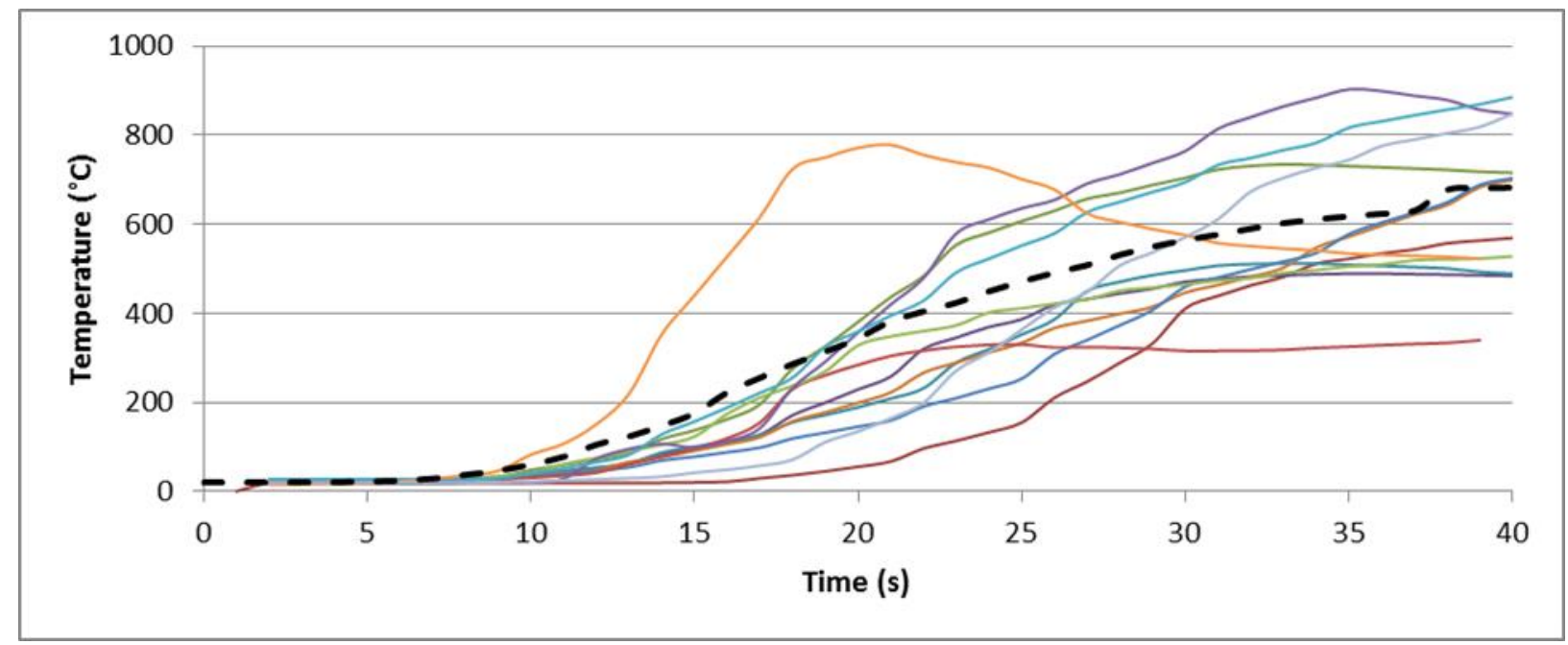

Figure B5 - Heating signature of the reaction zone with each herbal material present, and the average of all trials indicated by the black dashed line

The first step was to show that the heating signature produced by the propane torch was consistent between trials. Evaluation was carried out by placing the end of the torch approximately $2 \frac{1}{2}$ " from the T-junction, so that the tip of the inner blue cone of the flame was in direct contact with the quartz tube at the reaction zone. This was first carried out with no plant material present to allow for observation of the reproducibility of the heating process, and the temperature was monitored for 40 seconds in 10 replicate trials. The next step was to add the herbal material to ensure burning commenced and that a "smoking-like environment" could be established. Twelve pyrolysis trials of the six herbal substrates were performed with a burning time of 40 seconds and an $n=2$ for each species, and the heat signature was monitored. The propane torch produced a consistent heat signature as the heat was able to spread uniformly throughout the reaction zone. However, the independent herbal matrix pyrolysis trials displayed variability. This is due to the dependency on when and how that herbal material ignites and burns. The difference between these could be attributed to several hypothesized factors. The first is the dependency on where the plant material ignites and how the burning spreads. The 
thermocouple is centered in the reaction zone, but it is challenging to control the ignition and spread across the plant. Thus the proximity to the thermocouple may produce variability in the temperature reading between trials. Another possible factor is leaf size and density of the herbal substrate, which may cause a difference in actual amount of herbal material present as only the mass was held constant. Finally, different heat capacities may contribute as well, but these values are unknown.

Table B1 - Proposed identification of products observed in herbal material pyrolysis

\begin{tabular}{|c|}
\hline Herbal Material Pyrolytics Identification \\
\hline Phenol \\
\hline 4-Methylphenol (p-Cresol) \\
\hline 4-Hydroxypyridine (4-Pyridinol) \\
\hline 1,2-Benzenediol (Catechol) \\
\hline 2,3-dihydro-Benzofuran \\
\hline 1,2-Benzenediol (Hydroquinone) \\
\hline 2-methoxy-4-vinyl-Phenol \\
\hline 1,2-anhydro- $\beta$-D-Glucose \\
\hline (Z)-9-Octadecenenitrile (Oleanitrile) \\
\hline (Z)-9-Octadecenamide (Oleamide) \\
\hline
\end{tabular}

Each of the six herbal species was pyrolyzed in triplicate. If a chromatographic peak was observed within +/- $0.05 \mathrm{~min}$, it was deemed to be consistently produced. Ten chromatographic peaks were observed and tentatively identified. The tentative identifications of these ten products are shown in the table below. The products observed were consistent with previous plant matter pyrolysis studies. These studies have been conducted on different plant species, but display precedence of the varieties of compounds expected through the pyrolysis of plant material. In the 1970's, it was reported that plant pyrolysis produces a myriad of phenol and methoxyphenol type compounds and a study by Boon et al reported a major product of the Scirpus species to be 4vinyl-phenol, supporting reports of phenol-like pyrolytic products ${ }^{1-4}$. Cellulose is a large part of 
plant structure and degrades into several compounds, but one in specific is 1,6-anhydroglucose, which further breaks down into furan-like compounds ${ }^{5-6}$. Recent studies support furan-like product findings such as a 2005 study on tobacco pyrolysis, where the presence of phenol, pyridine, cresol, benzofuran, and dihydrobenzofuran were reported ${ }^{7}$. Two products observed in this paper, oleanitrile and oleamide, initially seemed surprising, but two recent studies show otherwise. Heo et al have shown that oleamide is present in many plant species, one specifically Ziziphus jujuba, and just this year, Jin et al shows that oleanitrile is present in at least the plant species of Dendrobium officinale and Dendrobium huoshanense $e^{8-9}$.

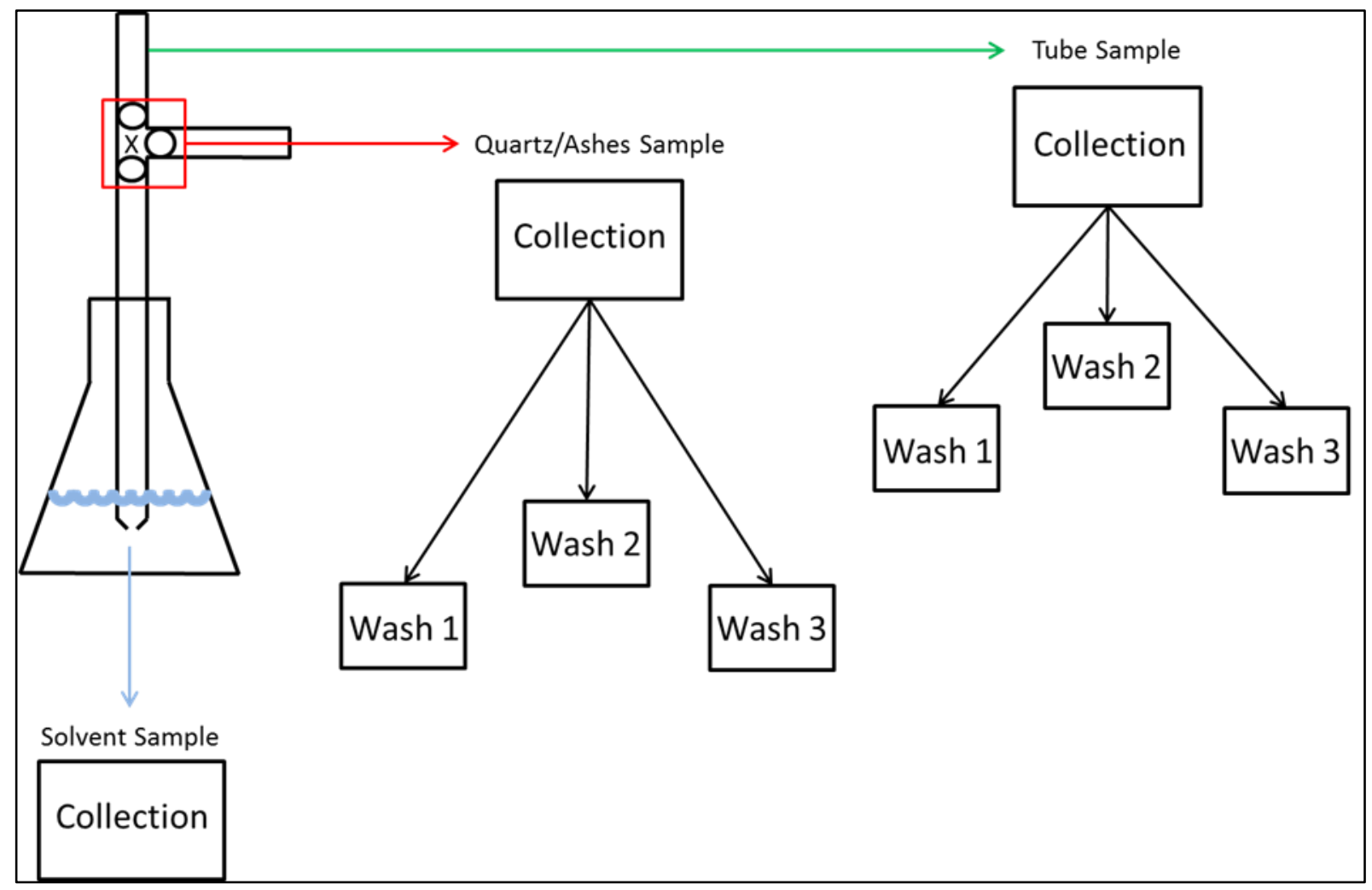

Figure B6 - Schematic of the exhaustive sampling of JWH-018 on Damiana to optimize the number of washes and replicates 


\section{References:}

1. Nagar, B.; Waight, E.; Meuzelaar, H.; Kistemaker, P., Studies on the structure and origin of soil humic acids by Curie point pyrolysis in direct combination with low-voltage mass spectrometry. Plant and Soil 1975, 43 (1-3), 681-685.

2. Haider, K.; Nagar, B.; Saiz, C.; Meuzelaar, H.; Martin, J., Studies on soil humic compounds, fungal melanins and model polymers by pyrolysis mass-spectrometry. In Soil organic matter studies, 1977.

3. Martin, F.; Saiz-Jimenez, C.; Cert, A., Pyrolysis-Gas Chromatography-Mass Spectrometry of Soil Humic Fractions: I. The Low Boiling Point Compounds 1. Soil Science Society of America Journal 1977, 41 (6), 1114-1118.

4. Boon, J. J.; Wetzel, R. G.; Godshalk, G. L., Pyrolysis mass spectrometry of some Scirpus species and their decomposition products. Limnology and Oceanography 1982, 27 (5), 839-848.

5. Shafizadeh, F. In Industrial pyrolysis of cellulosic materials, Appl. Polym. Symp, 1975; p 153.

6. Broido, A.; Evett, M.; Hodges, C. C., Yield of 1, 6-anhydro-3, 4-dideoxy- $\beta$-d-glycerohex-3-enopyranos-2-ulose (levoglucosenone) on the acid-catalyzed pyrolysis of cellulose and 1 , 6-anhydro- $\beta$-d-glucopyranose (levoglucosan). Carbohydrate Research 1975, 44 (2), 267-274.

7. Baker, R. R.; Bishop, L. J., The pyrolysis of non-volatile tobacco ingredients using a system that simulates cigarette combustion conditions. Journal of analytical and applied pyrolysis 2005, 74 (1-2), 145-170.

8. Heo, H.-J.; Park, Y.-J.; Suh, Y.-M.; Choi, S.-J.; Kim, M.-J.; Cho, H.-Y.; Chang, Y.-J.; Hong, B.; Kim, H.-K.; Kim, E., Effects of oleamide on choline acetyltransferase and cognitive activities. Bioscience, biotechnology, and biochemistry 2003, 67 (6), 1284-1291.

9. Jin, Q.; Jiao, C.; Sun, S.; Song, C.; Cai, Y.; Lin, Y.; Fan, H.; Zhu, Y., Metabolic analysis of medicinal dendrobium officinale and dendrobium huoshanense during different growth years. PloS one 2016, 11 (1), e0146607. 


\section{Appendix C}

\section{Supporting data and side project conducted, but not included in the chapter 2 manuscript.}

Percent Recovery Study of Pyrolysis Methodology:

The samples for pyrolysis on the herbal matrices were prepared by spraying the drug compound solution onto the matrix before allowing the solvent to evaporate to leave the drug behind. To evaluate the percent recovery, it was necessary to take the process step by step to determine any observed loss. As this project would eventually reach pyrolysis recovery, caffeine was chosen for this study because of its thermal stability. At this stage, breakdown was undesired to allow for quantitation of a single signal. Caffeine- $\mathrm{d}_{3}$ was used as an internal standard for quantitation. Establishing a known amount of drug sprayed onto sample was essential, and the first step was to determine the volume of solution dispensed per spray to allow for accurate concentration control.

This was examined by two methods. Both methods utilized the monitoring of weight difference to equate to volume using the density of water. Method 1 was to take the average weight difference of 10 sprays with 10 replicates. The data for this method is shown below in Table C1. Method 2 was to find the mass difference of each spray over the span of 25 sprays. The number of sprays was plotted vs the volume added, and the best fit line was found. The slope of this line is observed as the volume added per spray. The data and plot for method 2 are shown in Table C2 and Figure C1. Method 1 gave a volume per spray of $0.0421 \pm 0.00139 \mathrm{ml}$, and method 2 produced a volume of $0.0403 \mathrm{ml}$, which is slightly outside the range of method 1 by $0.00041 \mathrm{ml}$. For the remainder of the study, the Method 1 volume was used. 
Table C1 - Data for method 1 of the determination of volume per spray of the sprayer used for sample preparation. * : Density of water used was $0.9982 \mathrm{~g} / \mathrm{ml}$ due to a $20^{\circ} \mathrm{C}$ environment

\begin{tabular}{|c|c|c|c|}
\hline Initial Mass (g) & Final Mass (g) & Mass H2O Added (g) & Volume H2O Added (mL)* \\
\hline 10.07509 & 10.46708 & 0.39199 & 0.3927 \\
\hline 10.46708 & 10.89499 & 0.42791 & 0.4287 \\
\hline 11.05308 & 11.50102 & 0.44794 & 0.4487 \\
\hline 11.50102 & 11.92302 & 0.422 & 0.4228 \\
\hline 11.92302 & 12.33440 & 0.41138 & 0.4121 \\
\hline 12.33440 & 12.75682 & 0.42242 & 0.4232 \\
\hline 12.75682 & 13.17491 & 0.41809 & 0.4188 \\
\hline 13.17491 & 13.59617 & 0.42126 & 0.4220 \\
\hline 13.59617 & 14.01287 & 0.4167 & 0.4175 \\
\hline 14.01287 & 14.43478 & 0.42191 & 0.4227 \\
\hline & & Avg. Vol. of 10 & 0.4209 \\
\cline { 2 - 4 } & & sprays = \\
\cline { 2 - 4 } & & Std. Dev. = \\
\cline { 2 - 4 } & & Avg. Vol. per spray = & 0.0139 \\
\cline { 2 - 4 } & & Std. Dev. Per spray = & 0.0421 \\
\hline
\end{tabular}


Table C2 - The mass data for method 2 of the determination of the volume per spray.

\begin{tabular}{|c|c|c|c|}
\hline $\begin{array}{l}\text { Number of } \\
\text { Sprays }\end{array}$ & $\begin{array}{l}\text { Mass of } \\
\text { Vial }(g)\end{array}$ & $\begin{array}{c}\text { Total H2O Mass } \\
\text { Added (g) }\end{array}$ & $\begin{array}{c}\text { Total Volume H2O } \\
\text { Added }(\mathrm{mL})^{*}\end{array}$ \\
\hline 0 & 14.43204 & 0 & 0 \\
\hline 1 & 14.47436 & 0.04232 & 0.0424 \\
\hline 2 & 14.51576 & 0.08372 & 0.0839 \\
\hline 3 & 14.55515 & 0.12311 & 0.1233 \\
\hline 4 & 14.59753 & 0.16549 & 0.1658 \\
\hline 5 & 14.63892 & 0.20688 & 0.2073 \\
\hline 6 & 14.67970 & 0.24766 & 0.2481 \\
\hline 7 & 14.72175 & 0.28971 & 0.2902 \\
\hline 8 & 14.76194 & 0.3299 & 0.3305 \\
\hline 9 & 14.80091 & 0.36887 & 0.3695 \\
\hline 10 & 14.84014 & 0.4081 & 0.4088 \\
\hline 11 & 14.88021 & 0.44817 & 0.4490 \\
\hline 12 & 14.91791 & 0.48587 & 0.4867 \\
\hline 13 & 14.95873 & 0.52669 & 0.5276 \\
\hline 14 & 14.99902 & 0.56698 & 0.5680 \\
\hline 15 & 15.03883 & 0.60679 & 0.6079 \\
\hline 16 & 15.07958 & 0.64754 & 0.6487 \\
\hline 17 & 15.12238 & 0.69034 & 0.6916 \\
\hline 18 & 15.16474 & 0.7327 & 0.7340 \\
\hline 19 & 15.20188 & 0.76984 & 0.7712 \\
\hline 20 & 15.24196 & 0.80992 & 0.8114 \\
\hline 21 & 15.28286 & 0.85082 & 0.8524 \\
\hline 22 & 15.32499 & 0.89295 & 0.8946 \\
\hline 23 & 15.36353 & 0.93149 & 0.9332 \\
\hline 24 & 15.40079 & 0.96875 & 0.9705 \\
\hline 25 & 15.44315 & 1.01111 & 1.0129 \\
\hline
\end{tabular}




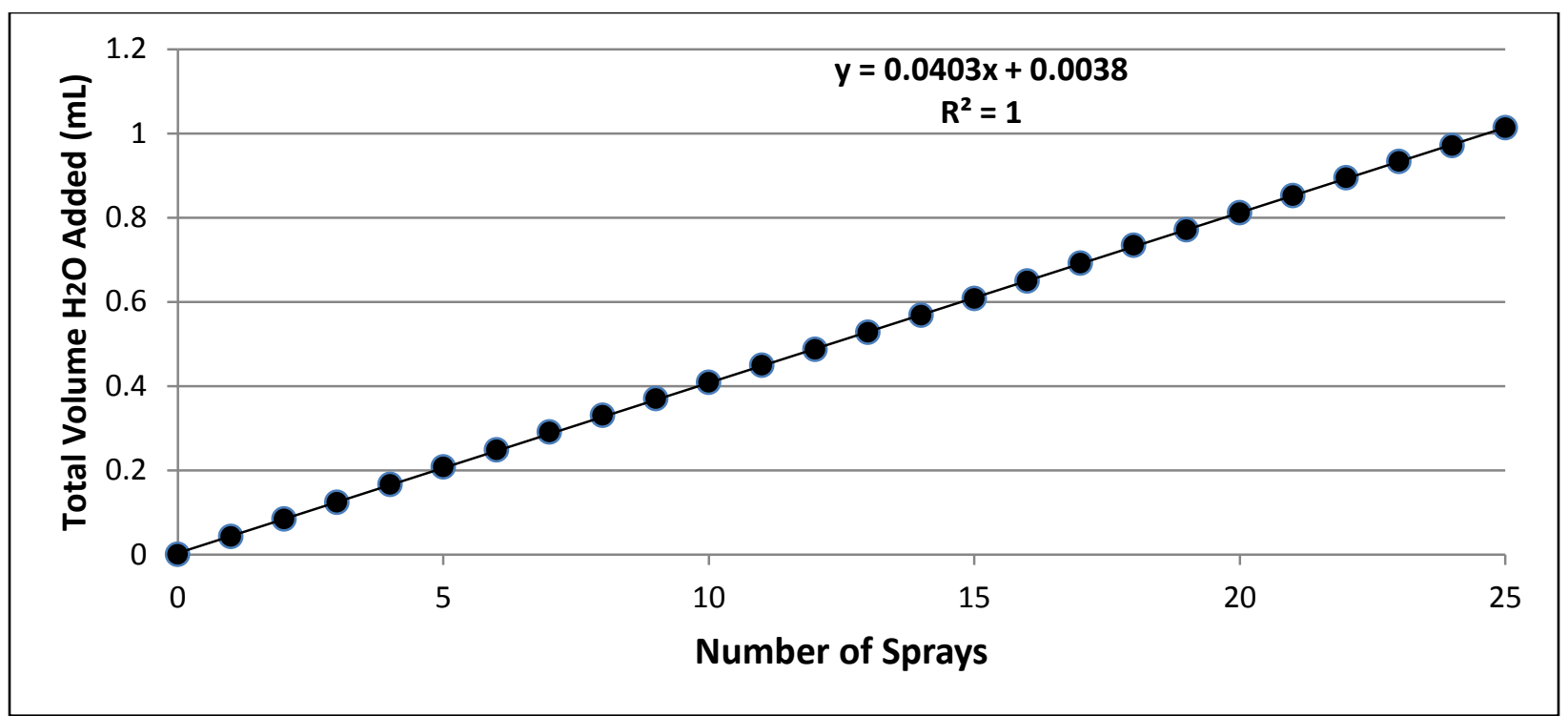

Figure C1 - Plot of volume added vs number of sprays with the equation of best fit line showing the volume per spray as the slope

The next step was to determine the mass of drug that reaches the herbal matrix. In the process of spraying the drug solution onto the matrix, two variables can lead to the loss of drug. One is due to the evaporation itself, and the second is to possible loss due to not actually being sprayed onto the herbal mixture. The spray is a mist, so solution could be left behind on weigh boat containing the matrix. The loss of drug for each step was evaluated to establish a drug concentration to be on the herbal matrix for pyrolysis after the sample preparation step.

First was the evaporation alone. This was carried out by removing the variable of the spreading mist by spraying the solution directly into a vial. The collected solution was allowed to evaporate, reconstituted, spiked with an internal standard and analyzed via GC/MS. The data for this step of the study is shown in Table C3. Sample calculations are shown below the table for SV1. The mean percent recovery of 69.5 will be used in following steps to calculate the amount of drug that should reach the herbal matrix, not barring loss due to mist spreading or being left behind on the weigh boat during sample preparation. 
Table C3 - Data for determining the loss of drug compound due to evaporation only.

\begin{tabular}{|c|c|c|c|c|c|c|c|c|c|}
\hline $\begin{array}{l}\text { Sample } \\
\text { Number }\end{array}$ & $\begin{array}{c}\text { Mass } \\
\text { Caffeine (mg) }\end{array}$ & $\begin{array}{c}\text { Reconstitution } \\
\text { Vol. }(\mathrm{mL})\end{array}$ & $\begin{array}{r}\text { Recoverd } \\
\text { Mass (mg) }\end{array}$ & $\begin{array}{c}\text { Percent } \\
\text { Recovery }\end{array}$ & $\begin{array}{l}\text { Analyzed } \\
\text { Vol. (mL) }\end{array}$ & $\begin{array}{l}\text { Mass Sample } \\
\text { Analyzed (mg) }\end{array}$ & Sample Area & $\begin{array}{c}\text { Mass Std. } \\
\text { Analyzed (mg) }\end{array}$ & $\begin{array}{c}\text { Std. Peak } \\
\text { Area }\end{array}$ \\
\hline SV1 & 20.0 & 4 & 16.5 & 82.3 & 1.5 & 6.17 & 530783232 & 0.400 & 34403080 \\
\hline SV2 & 20.0 & 4 & 12.8 & 64.2 & 1.5 & 4.81 & 580927552 & 0.400 & 48270736 \\
\hline SV3 & 20.1 & 4 & 12.1 & 60.2 & 1.5 & 4.54 & 517711232 & 0.400 & \begin{tabular}{|l|l|}
45623784 \\
\end{tabular} \\
\hline \begin{tabular}{|l|} 
SV4 \\
\end{tabular} & 19.9 & 4 & 12.2 & $\begin{array}{ll}61.4 \\
\end{array}$ & 1.5 & 4.58 & 452271360 & 0.400 & \begin{tabular}{|l|}
39483664 \\
\end{tabular} \\
\hline SV5 & 19.9 & 4 & 12.5 & 63.1 & 1.5 & 4.71 & 423705344 & 0.400 & 36016228 \\
\hline \begin{tabular}{|l|} 
SV6 \\
\end{tabular} & 19.9 & 4 & 13.4 & 67.5 & 1.5 & 5.04 & 432123520 & 0.400 & 34295264 \\
\hline SV7 & 19.9 & 4 & 14.5 & 73.0 & 1.5 & 5.45 & 475603680 & 0.400 & \begin{tabular}{|l|}
34917392 \\
\end{tabular} \\
\hline \begin{tabular}{|l|} 
SV8 \\
\end{tabular} & 20.1 & 4 & 13.5 & 67.4 & 1.5 & 5.08 & 414302976 & 0.400 & 32622774 \\
\hline \begin{tabular}{|l|} 
SV9 \\
\end{tabular} & 20.1 & 4 & 15.0 & 74.5 & 1.5 & 5.62 & 434313696 & 0.400 & 30929504 \\
\hline SV10 & 20.1 & 4 & 14.5 & 72.1 & 1.5 & 5.43 & 428123840 & 0.400 & 31511178 \\
\hline \multirow[t]{4}{*}{ SV11 } & 20.0 & 4 & 15.8 & 78.9 & 1.5 & 5.91 & 408213984 & 0.400 & 27609438 \\
\hline & & Mean $=$ & 13.9 & 69.5 & Mean $=$ & 5.21 & & & \\
\hline & & Std. Dev. = & 1.5 & 7.2 & Std. Dev. = & 0.55 & & & \\
\hline & & Range = & $12.4-15.4$ & $62.3-76.7$ & Range = & $4.66-5.76$ & & & \\
\hline
\end{tabular}

$$
\text { Mass Sample Analyzed }=\left(\frac{\text { Mass Std.Analyzed }}{\text { Std.Peak Area }}\right) X(\text { Sample Area })
$$

$$
\text { Mass SV1 Analyzed }=\left(\frac{0.400 \mathrm{mg}}{34403080}\right) X(53078232)=6.17 \mathrm{mg}
$$

Recovered Mass $=\left(\frac{\text { Mass Sample Analyzed }}{\text { Analyzed Volume }}\right) \times$ Reconstitution Volume

$$
\begin{gathered}
\text { Recovered SV1 Mass }=\left(\frac{6.17 \mathrm{mg}}{1.5 \mathrm{ml}}\right) \times 4 \mathrm{ml}=16.5 \mathrm{mg} \\
\text { Percent Recovery }=\left(\frac{\text { Recovered Mass }}{\text { Caffeine Mass }}\right) \times 100 \% \\
\text { SV1 Percent Recovery }=\left(\frac{16.5 \mathrm{mg}}{20.0 \mathrm{mg}}\right) \times 100 \%=82.3 \%
\end{gathered}
$$

Next step was to determine the average amount of drug lost during the sample preparation step of spraying the herbal matrix. This could be done in two ways: 1) spray smaller volumes in multiple cycles to more precisely spray onto the matrix and allow evaporation in 
between each 2) spray the full volume at once and allow for one evaporation step. After the drug was displaced onto the herbal matrix and dried, it was soaked in fresh solvent, spiked with internal standard and analyzed via GC/MS. For the calculations, the percent recovery was based off of the possible $100 \%$ being $69.5 \%$ of the total mass due to the evaporation loss from the previous step. The data for both applications are shown below in Table C4 and Table C5, and a sample calculation of P-SP1 and P-SA1 are shown below. It was observed that the technique of spraying the entire drug solution volume onto the herbal matrix at once left a higher amount of drug on the matrix at a mean of $75.7 \%$.

Table C4 - Data for the determination of loss due to the spraying of drug solution onto the herbal matrix by periodic sprays

\begin{tabular}{|c|c|c|c|c|c|c|c|c|c|c|}
\hline $\begin{array}{c}\text { Sample } \\
\text { Number }\end{array}$ & $\begin{array}{c}\text { Mass } \\
\text { Caffeine (mg) }\end{array}$ & $\begin{array}{c}\text { Mass } \\
\text { Delivered (mg) }\end{array}$ & $\begin{array}{c}\text { Reconstitution } \\
\text { Vol. (mL) }\end{array}$ & $\begin{array}{r}\text { Recoverd } \\
\text { Mass (mg) }\end{array}$ & $\begin{array}{c}\text { Percent } \\
\text { Recovery }\end{array}$ & $\begin{array}{l}\text { Analyzed } \\
\text { Vol. (mL) }\end{array}$ & $\begin{array}{l}\text { Sample Mass } \\
\text { Analyzed (mg) }\end{array}$ & Sample Area & $\begin{array}{c}\text { Mass Std. } \\
\text { Analyzed (mg) }\end{array}$ & $\begin{array}{c}\text { Std. Peak } \\
\text { Area }\end{array}$ \\
\hline P-SP1 & 20.0 & 13.9 & 6 & 8.73 & 62.8 & 1.5 & 2.18 & 160872448 & 0.400 & 29477514 \\
\hline P-SP2 & 19.9 & 13.8 & 6 & 6.97 & 50.4 & 1.5 & 1.74 & 146505280 & 0.400 & 33609992 \\
\hline P-SP3 & 19.9 & 13.8 & 6 & 6.54 & 47.3 & 1.5 & 1.63 & 130672840 & 0.400 & 31980474 \\
\hline P-SP4 & 20.0 & 13.9 & 6 & 7.16 & 51.5 & 1.5 & 1.79 & 132992016 & 0.400 & 29715754 \\
\hline P-SP5 & 20.0 & 13.9 & 6 & 7.80 & 56.1 & 1.5 & 1.95 & 137002128 & 0.400 & 28112380 \\
\hline P-SP6 & 20.1 & 14.0 & 6 & 8.09 & 57.9 & 1.5 & 2.02 & 139769264 & 0.400 & 27630382 \\
\hline P-SP7 & 19.9 & 13.8 & 6 & 7.17 & 51.8 & 1.5 & 1.79 & 130919344 & 0.400 & 29215846 \\
\hline P-SP8 & 20.0 & 13.9 & 6 & 8.07 & 58.1 & 1.5 & 2.02 & 137043904 & 0.400 & 27173758 \\
\hline P-SP9 & 20.1 & 14.0 & 6 & 8.30 & 59.4 & 1.5 & 2.07 & 131053472 & 0.400 & 25266474 \\
\hline P-SP10 & 19.9 & 13.8 & 6 & 7.79 & 56.3 & 1.5 & 1.95 & 132081552 & 0.400 & 27121258 \\
\hline \multirow{3}{*}{\multicolumn{3}{|c|}{ Delivered Mass $=$ Caffeine Mass x $69.5 \%$}} & Mean = & 7.66 & 55.2 & Mean = & 1.92 & & & \\
\hline & & & Std. Dev. = & 0.68 & 4.8 & Std. Dev. = & 0.17 & & & \\
\hline & & & Range $=$ & $6.98-8.34$ & $47.3-62.8$ & Range $=$ & $1.75-2.09$ & & & \\
\hline
\end{tabular}

$$
\begin{gathered}
\text { Mass Sample Analyzed }=\left(\frac{\text { Mass Std.Analyzed }}{\text { Std.Peak Area }}\right) X(\text { Sample Area }) \\
\text { Mass } P-S P 1 \text { Analyzed }=\left(\frac{0.400 \mathrm{mg}}{29477514}\right) X(160872448)=2.18 \mathrm{mg} \\
\text { Recovered Mass }=\left(\frac{\text { Mass Sample Analyzed }}{\text { Analyzed Volume }}\right) X \text { Reconstitution Volume }
\end{gathered}
$$




$$
\begin{gathered}
\text { Recovered } P-S P 1 \text { Mass }=\left(\frac{2.18 \mathrm{mg}}{1.5 \mathrm{ml}}\right) \times 6 \mathrm{ml}=8.73 \mathrm{mg} \\
\text { Percent Recovery }=\left(\frac{\text { Recovered Mass }}{\text { Delivered Mass }}\right) \times 100 \% \\
P-S P 1 \text { Percent Recovery }=\left(\frac{8.73 \mathrm{mg}}{13.9 \mathrm{mg}}\right) \times 100 \%=62.8 \%
\end{gathered}
$$

\begin{tabular}{|c|c|c|c|c|c|c|c|c|c|c|}
\hline $\begin{array}{l}\text { Sample } \\
\text { Number }\end{array}$ & $\begin{array}{c}\text { Mass } \\
\text { Caffeine (mg) }\end{array}$ & $\begin{array}{c}\text { Delivered } \\
\text { Mass (mg) }\end{array}$ & $\begin{array}{l}\text { Reconstitution } \\
\text { Vol. }(\mathbf{m L})\end{array}$ & $\begin{array}{l}\text { Recoverd } \\
\text { Mass (mg) }\end{array}$ & $\begin{array}{c}\text { Percent } \\
\text { Recovery }\end{array}$ & $\begin{array}{l}\text { Analyzed } \\
\text { Vol. (mL) }\end{array}$ & $\begin{array}{l}\text { Sample Mass } \\
\text { Analyzed (mg) }\end{array}$ & Sample Area & $\begin{array}{c}\text { Mass Std. } \\
\text { Analyzed (mg) }\end{array}$ & $\begin{array}{c}\text { Std. Peak } \\
\text { Area }\end{array}$ \\
\hline P-SA1 & 20.1 & 14.0 & 6 & 9.80 & 70.1 & 1.5 & 2.45 & 177612592 & 0.400 & 29001432 \\
\hline P-SA2 & 20.0 & 13.9 & 6 & 10.16 & 73.1 & 1.5 & 2.54 & 176853472 & 0.400 & 27857740 \\
\hline P-SA3 & 20.1 & 14.0 & 6 & 10.01 & 71.6 & 1.5 & 2.50 & 180016800 & 0.400 & 28779008 \\
\hline P-SA4 & 20.0 & 13.9 & 6 & 10.17 & 73.2 & 1.5 & 2.54 & 189699520 & 0.400 & 29833854 \\
\hline P-SA5 & 20.0 & 13.9 & 6 & 8.68 & 62.4 & 1.5 & 2.17 & 181174656 & 0.400 & 33405652 \\
\hline P-SA6 & 20.1 & 14.0 & 6 & 9.91 & 70.9 & 1.5 & 2.48 & 175969472 & 0.400 & 28413448 \\
\hline P-SA7 & 20.1 & 14.0 & 6 & 13.81 & 98.9 & 1.5 & 3.45 & 192910896 & 0.400 & 22345990 \\
\hline P-SA8 & 19.9 & 13.8 & 6 & 11.45 & 82.8 & 1.5 & 2.86 & 171856880 & 0.400 & 24013084 \\
\hline P-SA9 & 20.1 & 14.0 & 6 & 11.36 & 81.3 & 1.5 & 2.84 & 170186608 & 0.400 & 23964374 \\
\hline P-SA10 & 20.0 & 13.9 & 6 & 10.12 & 72.8 & 1.5 & 2.53 & 172357584 & 0.400 & 27248658 \\
\hline \multirow{3}{*}{\multicolumn{3}{|c|}{ Delivered Mass $=$ Caffeine Mass $\times 69.5 \%$}} & Mean $=$ & 10.5 & 75.7 & Mean = & 2.64 & & & \\
\hline & & & Std. Dev. = & 1.4 & 9.9 & Std. Dev. = & 0.35 & & & \\
\hline & & & Range $=$ & $9.1-11.9$ & $62.4-98.9$ & Range $=$ & $2.29-2.99$ & & & \\
\hline
\end{tabular}

Table C5 - Data for the determination of loss due to the spraying of drug solution onto the herbal matrix all at once

$$
\begin{aligned}
& \text { Mass Sample Analyzed }=\left(\frac{\text { Mass Std.Analyzed }}{\text { Std.Peak Area }}\right) \times(\text { Sample Area }) \\
& \text { Mass P-SA1 Analyzed }=\left(\frac{0.400 \mathrm{mg}}{29001432}\right) \times(177612592)=2.45 \mathrm{mg}
\end{aligned}
$$$$
\text { Recovered Mass }=\left(\frac{\text { Mass Sample Analyzed }}{\text { Analyzed Volume }}\right) \times \text { Reconstitution Volume }
$$

$$
\text { Recovered } P-S A 1 \text { Mass }=\left(\frac{2.45 \mathrm{mg}}{1.5 \mathrm{ml}}\right) \times 6 \mathrm{ml}=9.80 \mathrm{mg}
$$




$$
\begin{gathered}
\text { Percent Recovery }=\left(\frac{\text { Recovered Mass }}{\text { Delivered Mass }}\right) \times 100 \% \\
P-S A 1 \text { Percent Recovery }=\left(\frac{9.80 \mathrm{mg}}{14.0 \mathrm{mg}}\right) \times 100 \%=70.1 \%
\end{gathered}
$$

Lastly, the prepared samples were pyrolyzed to determine the recovery of the burning methodology. The calculations were based on the assumptions of the previous two steps; $69.5 \%$ retention from evaporation and $75.7 \%$ of that retention onto the herbal matrix. Samples were prepped using the full volume at once technique, and the recovery was evaluated. After a burning trial, each collected sample (quartz, tube and flask) was quantified and added together for a total collection of drug. The data is presented within Table C6, and a sample calculation is below.

Table C6 - Data for the determination of recovery of overall sample preparation, pyrolysis and

\begin{tabular}{|c|c|c|c|c|c|c|c|c|c|c|c|}
\hline $\begin{array}{c}\text { Trial } \\
\text { Number }\end{array}$ & $\begin{array}{c}\text { Mass } \\
\text { Caffeine (mg) }\end{array}$ & $\begin{array}{c}\text { Delivered } \\
\text { Mass (mg) }\end{array}$ & $\begin{array}{l}\text { Collected } \\
\text { Sample }\end{array}$ & $\begin{array}{c}\text { Reconstitution } \\
\text { Vol. }(\mathrm{mL})\end{array}$ & $\begin{array}{c}\text { Recoverd } \\
\text { Mass (mg) }\end{array}$ & Percent Recovery & $\begin{array}{l}\text { Analyzed } \\
\text { Vol. (mL) }\end{array}$ & $\begin{array}{l}\text { Sample Mass } \\
\text { Analyzed (mg) }\end{array}$ & Sample Area & $\begin{array}{c}\text { Mass Std. } \\
\text { Analyzed (mg) }\end{array}$ & $\begin{array}{c}\text { Std. Peak } \\
\text { Area }\end{array}$ \\
\hline \multirow{3}{*}{1} & \multirow{3}{*}{20.0} & \multirow{3}{*}{10.52} & QW1 & 1 & 0.00308 & 0.0293 & 0.3 & 0.000925 & 19049 & 0.100 & 2059023 \\
\hline & & & $\mathrm{T} 1$ & 3 & 1.15 & 10.9 & 1.5 & 0.575961 & 2414587 & 0.400 & 1676910 \\
\hline & & & F1 & 3 & 1.53 & 14.5 & 1.5 & 0.763497 & 3111577 & 0.400 & 1630171 \\
\hline & & & & Total $=$ & 2.68 & 25.5 & & & & & \\
\hline \multirow{3}{*}{2} & \multirow{3}{*}{20.1} & \multirow{3}{*}{10.57} & QW2 & 1 & 0.0217 & 0.205 & 0.3 & 0.00651 & 159126 & 0.100 & 2444907 \\
\hline & & & $\mathrm{T} 2$ & 3 & 1.80 & 17.0 & 1.5 & 0.90068 & 4212331 & 0.400 & 1870735 \\
\hline & & & $\mathrm{F} 2$ & 3 & 2.67 & 25.3 & 1.5 & 1.33561 & 6200113 & 0.400 & 1856858 \\
\hline & & & & Total $=$ & 4.49 & 42.5 & & & & & \\
\hline \multirow{3}{*}{3} & \multirow{3}{*}{19.9} & \multirow{3}{*}{10.47} & QW3 & 1 & 0.226 & 2.16 & 0.3 & 0.0678 & 1614094 & 0.100 & 2381402 \\
\hline & & & $\mathrm{T} 3$ & 3 & 1.90 & 18.1 & 1.5 & 0.9495 & 5359954 & 0.400 & 2258061 \\
\hline & & & F3 & 3 & 4.40 & 42.0 & 1.5 & 2.2003 & 13377304 & 0.400 & 2431886 \\
\hline & & & & Total $=$ & 6.53 & 62.3 & & & & & \\
\hline \multirow{3}{*}{4} & \multirow{3}{*}{20.1} & \multirow{3}{*}{10.57} & QW4 & 1 & 0.140 & 1.32 & 0.3 & 0.0419 & 1097624 & 0.100 & 2620995 \\
\hline & & & $\mathrm{T} 4$ & 3 & 1.34 & 12.7 & 1.5 & 0.6719 & 3866208 & 0.400 & 2301586 \\
\hline & & & $\mathrm{F} 4$ & 3 & 3.21 & 30.4 & 1.5 & 1.6070 & 9269358 & 0.400 & 2307316 \\
\hline & & & & Total $=$ & 4.70 & 44.4 & & & & & \\
\hline \multirow{3}{*}{5} & \multirow{3}{*}{19.9} & \multirow{4}{*}{10.47} & QW5 & 1 & 0.291 & 2.8 & 0.3 & 0.0872 & 2119155 & 0.100 & 2429536 \\
\hline & & & $\mathrm{T} 5$ & 3 & 1.48 & 14.1 & 1.5 & 0.7392 & 4573155 & 0.400 & 2474586 \\
\hline & & & F5 & 3 & 4.65 & 44.4 & 1.5 & 2.3229 & 15288100 & 0.400 & 2632636 \\
\hline & & & & Total $=$ & 6.41 & 61.3 & & & & & \\
\hline \multicolumn{4}{|c|}{ Delivered Mass $=$ Caffeine Mass $\times 69.5 \% \times 75.7 \%$} & Average $=$ & 4.96 & 47.20 & & & & & \\
\hline
\end{tabular}
sample collection process

$$
\text { Mass Sample Analyzed }=\left(\frac{\text { Mass Std.Analyzed }}{\text { Std.Peak Area }}\right) X(\text { Sample Area })
$$


Mass QW3 Analyzed $=\left(\frac{0.100 \mathrm{mg}}{2381402}\right) X(159126)=0.0678 \mathrm{mg}$

Mass T3 Analyzed $=\left(\frac{0.400 \mathrm{mg}}{2258061}\right) \times(5359954)=0.950 \mathrm{mg}$

Mass F3 Analyzed $=\left(\frac{0.400 \mathrm{mg}}{2431886}\right) \times(13377304)=2.20 \mathrm{mg}$

Recovered Mass $=\left(\frac{\text { Mass Sample Analyzed }}{\text { Analyzed Volume }}\right) \times$ Reconstitution Volume

Recovered QW3 Mass $=\left(\frac{0.0678 \mathrm{mg}}{0.3 \mathrm{ml}}\right) \times 1 \mathrm{ml}=0.226 \mathrm{mg}$

$$
\begin{aligned}
& \text { Recovered T3 Mass }=\left(\frac{0.950 \mathrm{mg}}{1.5 \mathrm{ml}}\right) \times 1 \mathrm{ml}=1.90 \mathrm{mg} \\
& \text { Recovered F3 Mass }=\left(\frac{2.20 \mathrm{mg}}{1.5 \mathrm{ml}}\right) \times 1 \mathrm{ml}=4.40 \mathrm{mg}
\end{aligned}
$$

Percent Recovery $=\left(\frac{\text { Total Recovered Mass }}{\text { Delivered Mass }}\right) \times 100 \%$

Sample 1 Percent Recovery $=\left(\frac{6.53 \mathrm{mg}}{10.47 \mathrm{mg}}\right) \times 100 \%=62.3 \%$ 
Proposed Mechanisms of the three major breakdown trends observed:

The chemistry that takes place within the pyrolytic environment is difficult to fully understand. It has long been thought that at extreme temperatures the reactions proceed through radical reactions, especially the ring expansion process ${ }^{1-3}$. However, this has not been proven through the use of labelled carbon or deuterium studies. For this to occur, the compounds involved in the reaction would be forced to initiate the production of a free radical instead of more energy favorable electron pushing chemistry while holding true to neutral compounds ${ }^{4}$. The common radical hypothesized to be produced is a hydrogen radical, which can take approximately $52 \mathrm{kcal} / \mathrm{mol}$ to produce ${ }^{5}$, where hydride shifts are typically closer to $10 \mathrm{kcal} / \mathrm{mol}$ depending on the state of the carbocation ${ }^{6}$. The mechanism of pyrolytic breakdown on illicit drugs is an area largely lacking in experimental data and research. For the current research, the proposed mechanisms take the approach of simple electron pushing and hydride shifts as an explanation for the three observed trends. This is based on this type of chemical reactions being more energy favorable in lieu of producing free radicals. It is not to say that free radicals could not be produced and additional mechanism are possible, especially in the extremely high temperatures, however, it is hypothesized that these such processes would occur before such extreme temperatures would be reached. 


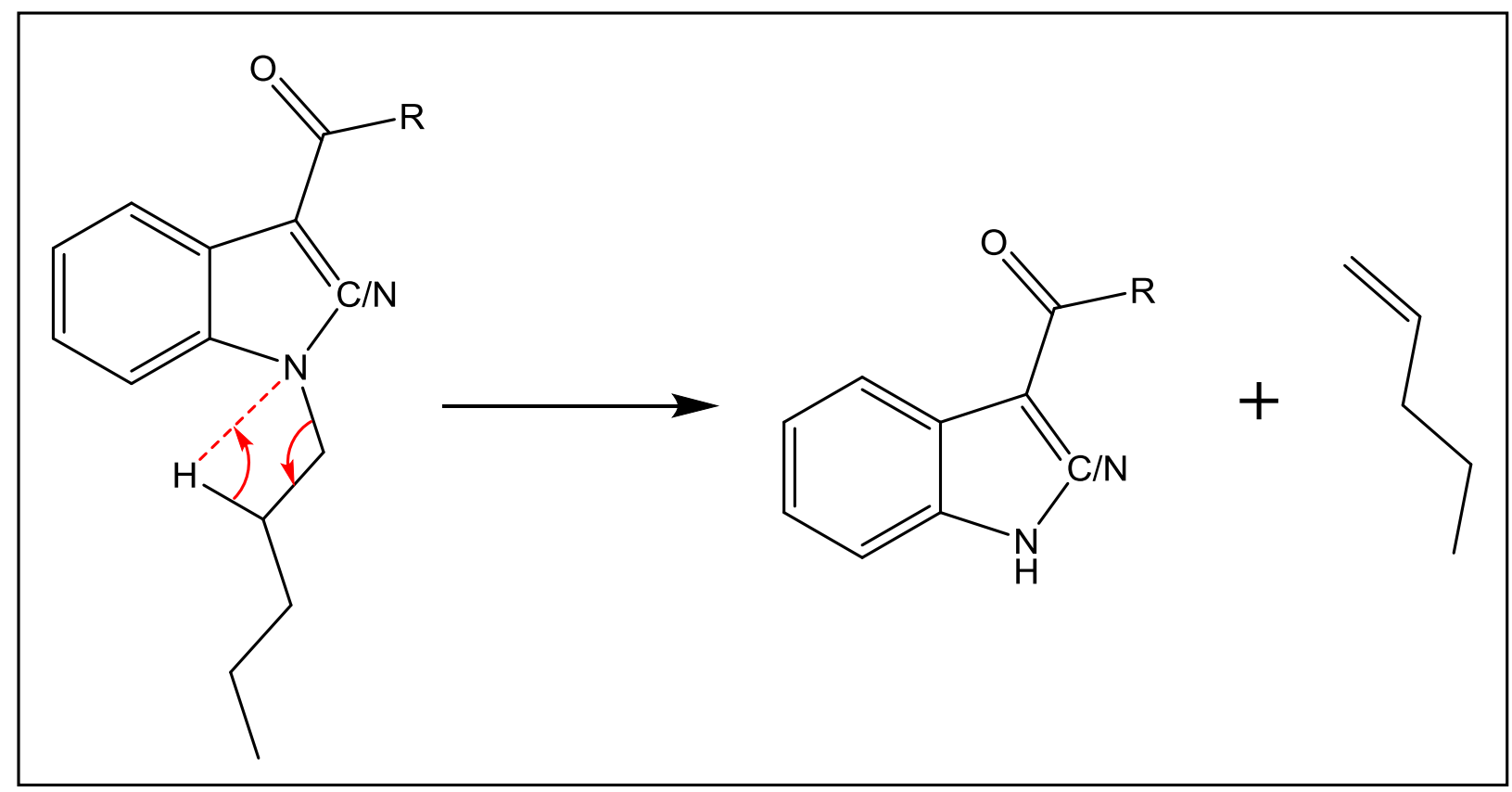

Figure $\mathrm{C} 2$ - Proposed mechanism of a pyrolytic cleavage at the nitrogen within the indole ring (or could be an indazole). 


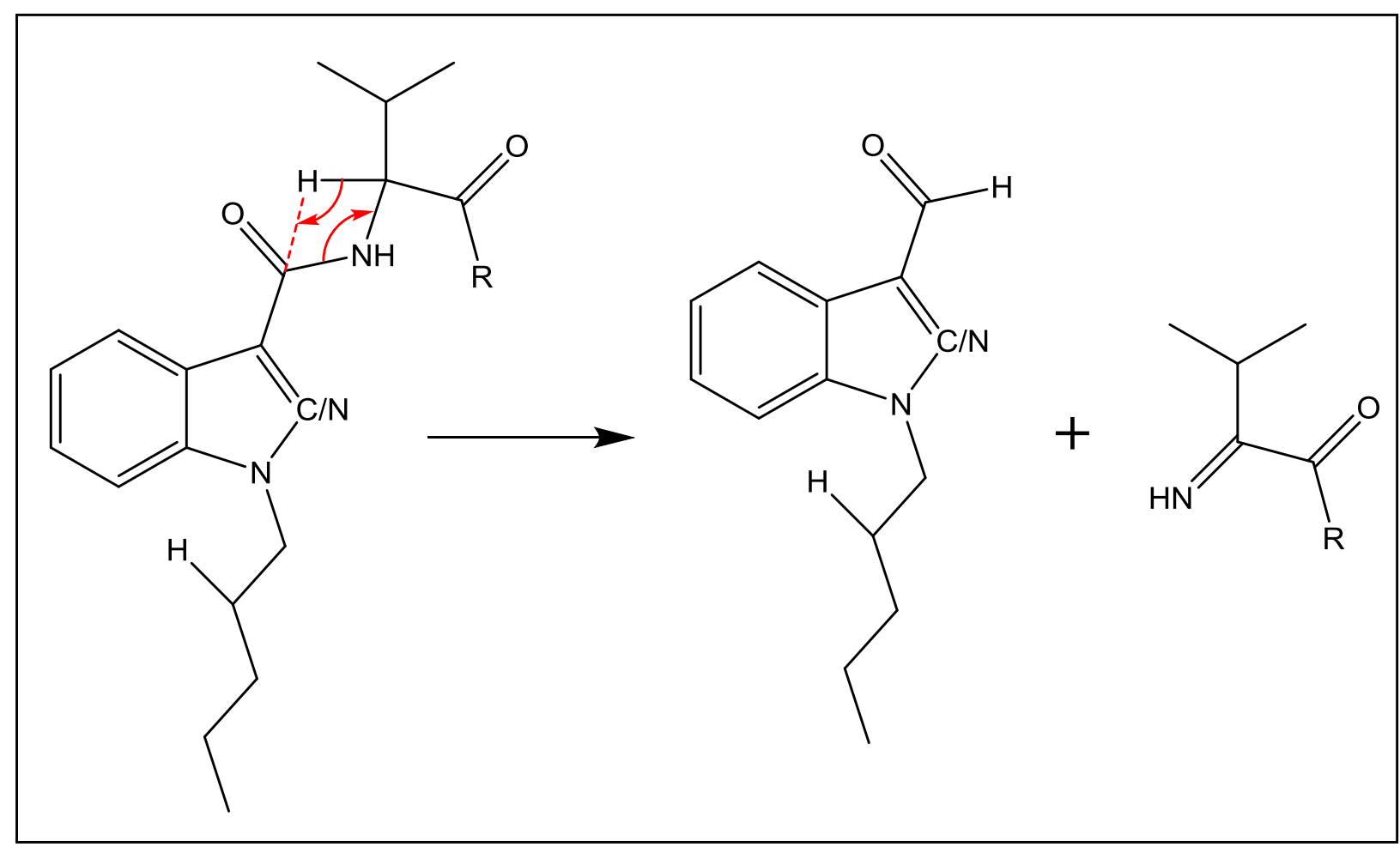

Figure C3 - Proposed mechanism of a pyrolytic cleavage of the central carbonyl group on the non-indole/indazole side. 


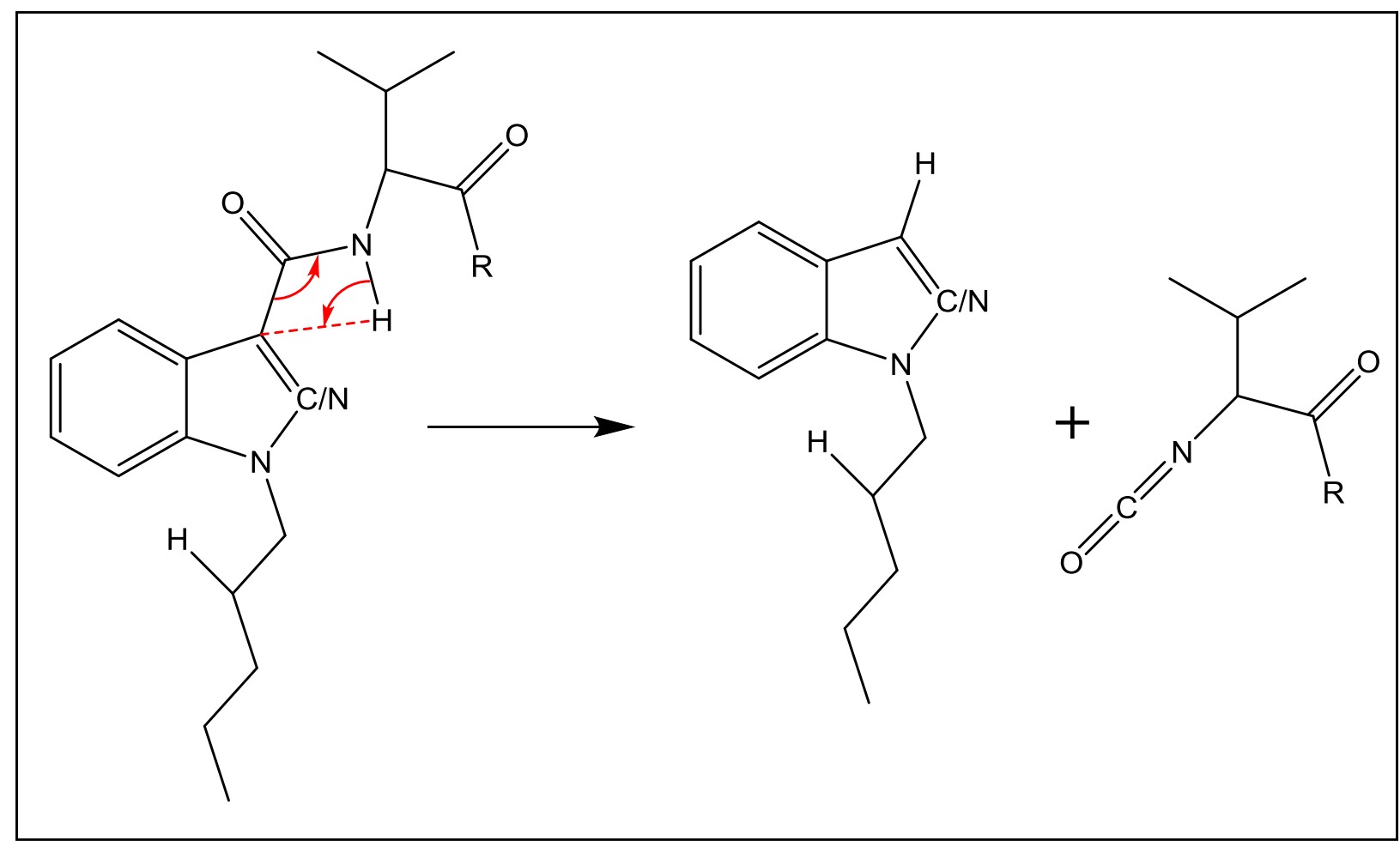

Figure $\mathrm{C} 4$ - Proposed mechanism of a pyrolytic cleavage of the central carbonyl group on the indole/indazole side.

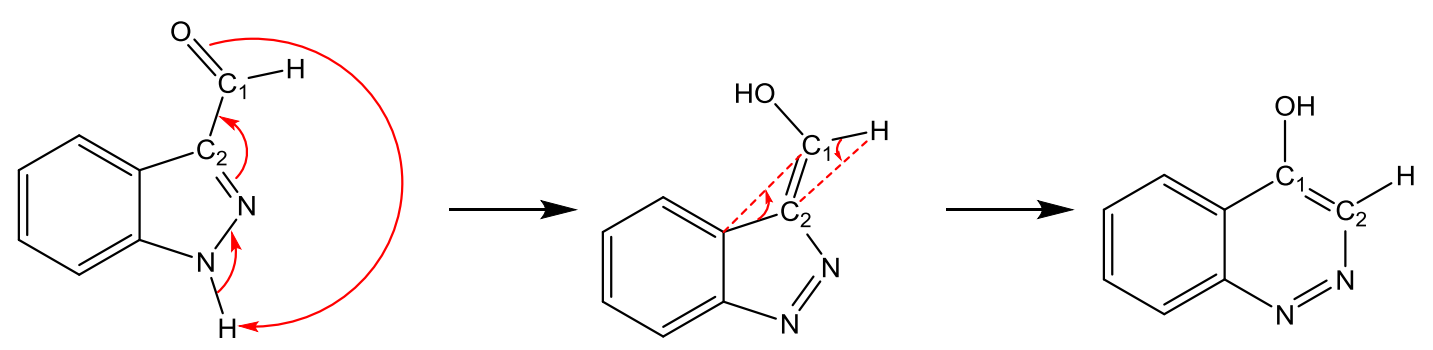

After mechanisms from Figures $\mathrm{C} 2$ and $\mathrm{C} 3$

OR<smiles></smiles>

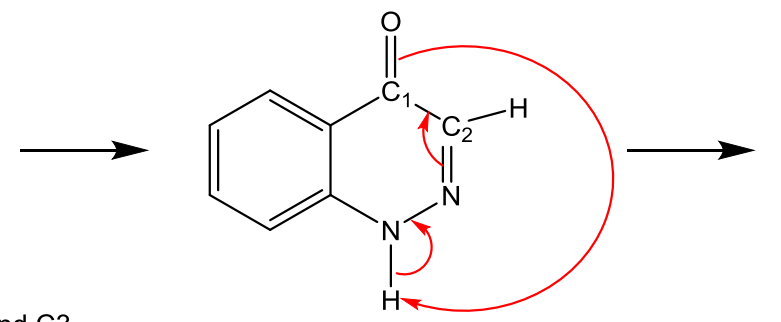<smiles></smiles>

After mechanisms from Figures $\mathrm{C} 2$ and $\mathrm{C} 3$

Figure C5 - Proposed mechanism of a ring expansion from indazole to cinnoline 
Parent Synthetic Cannabinoid to its Proposed Pyrolytic Products Breakdowns:

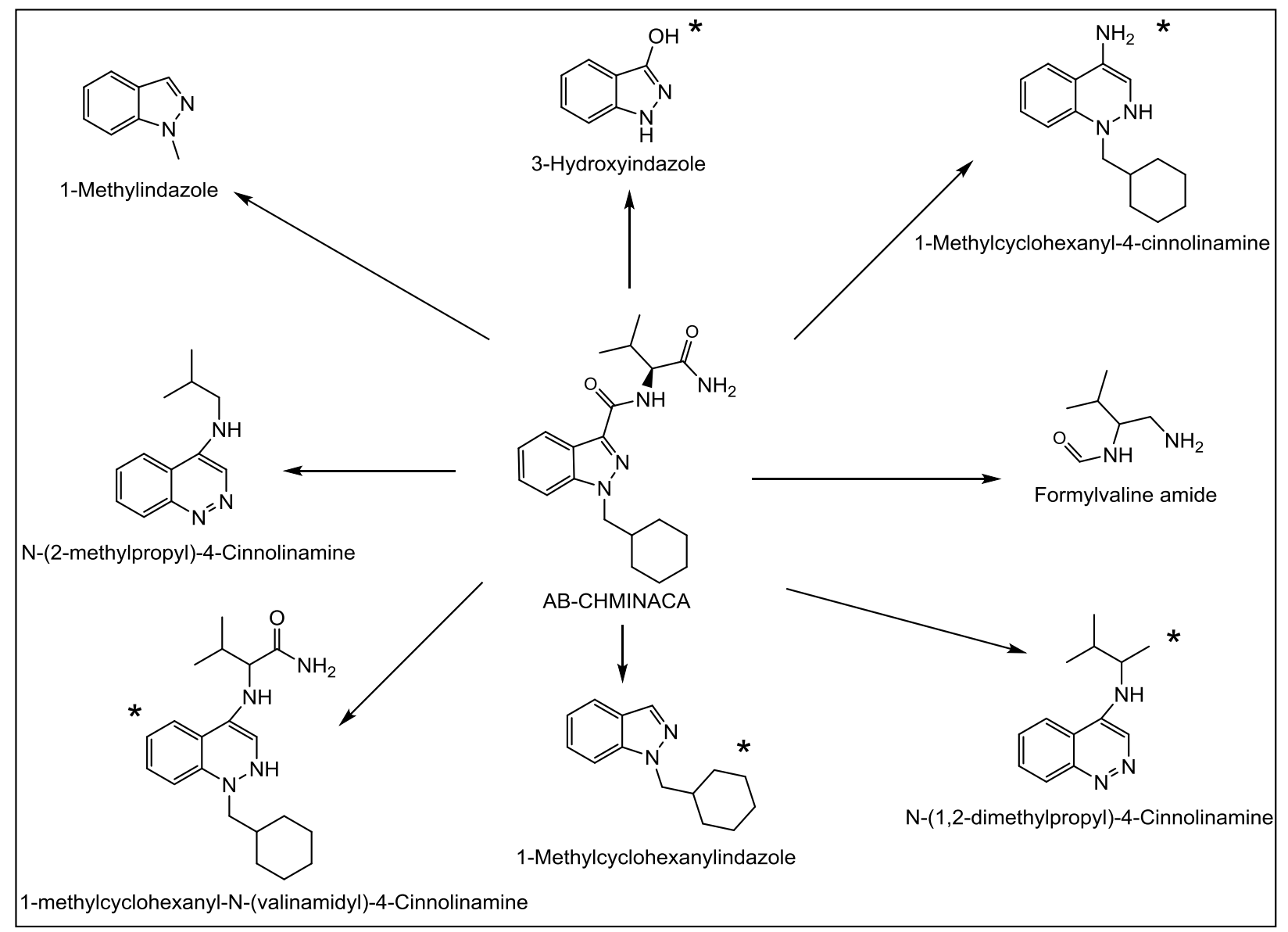

Figure C7 - Thermal degradation breakdown of AB-CHMINACA to its proposed observed pyrolytic products 


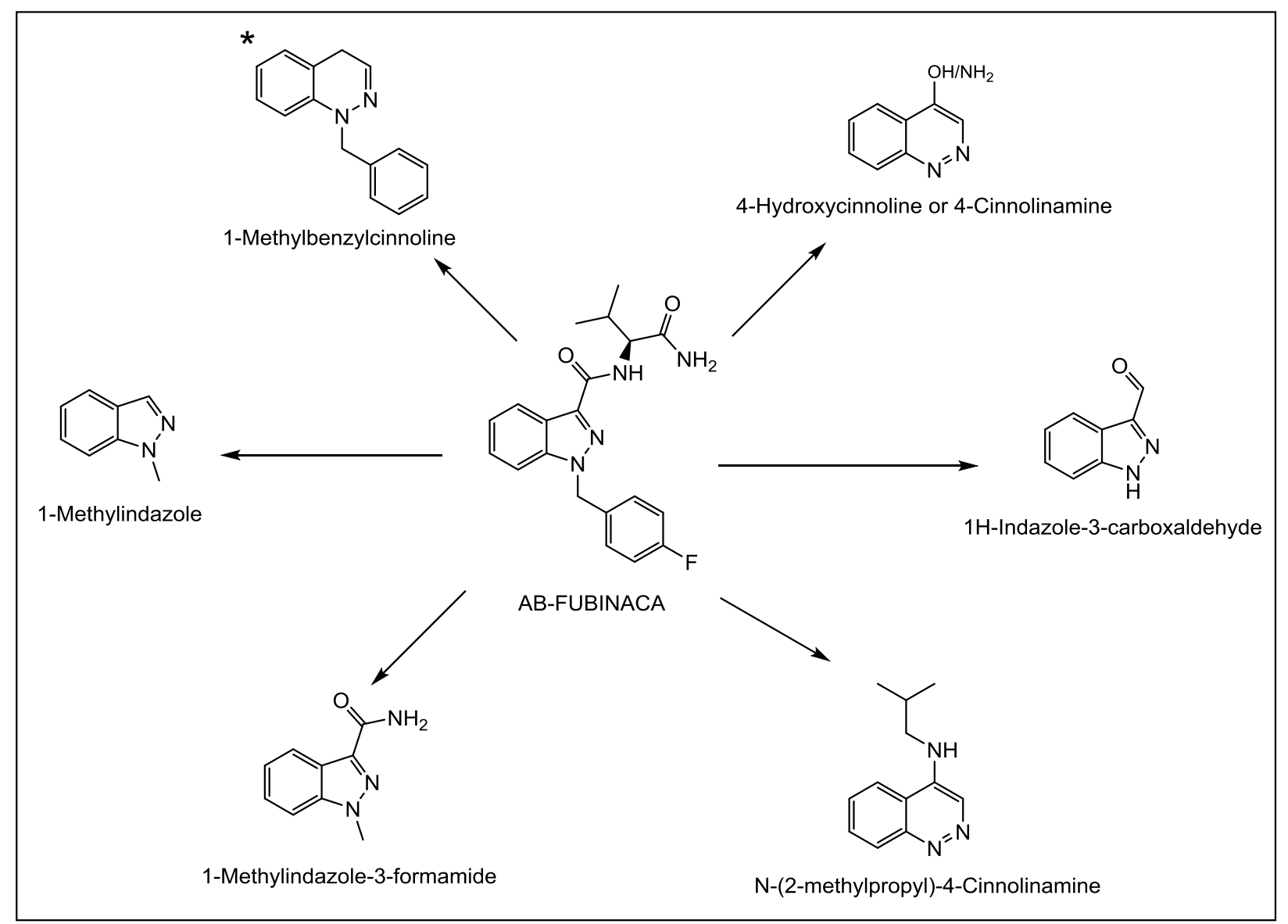

Figure C8 - Thermal degradation breakdown of AB-FUBINACA to its proposed observed pyrolytic products 


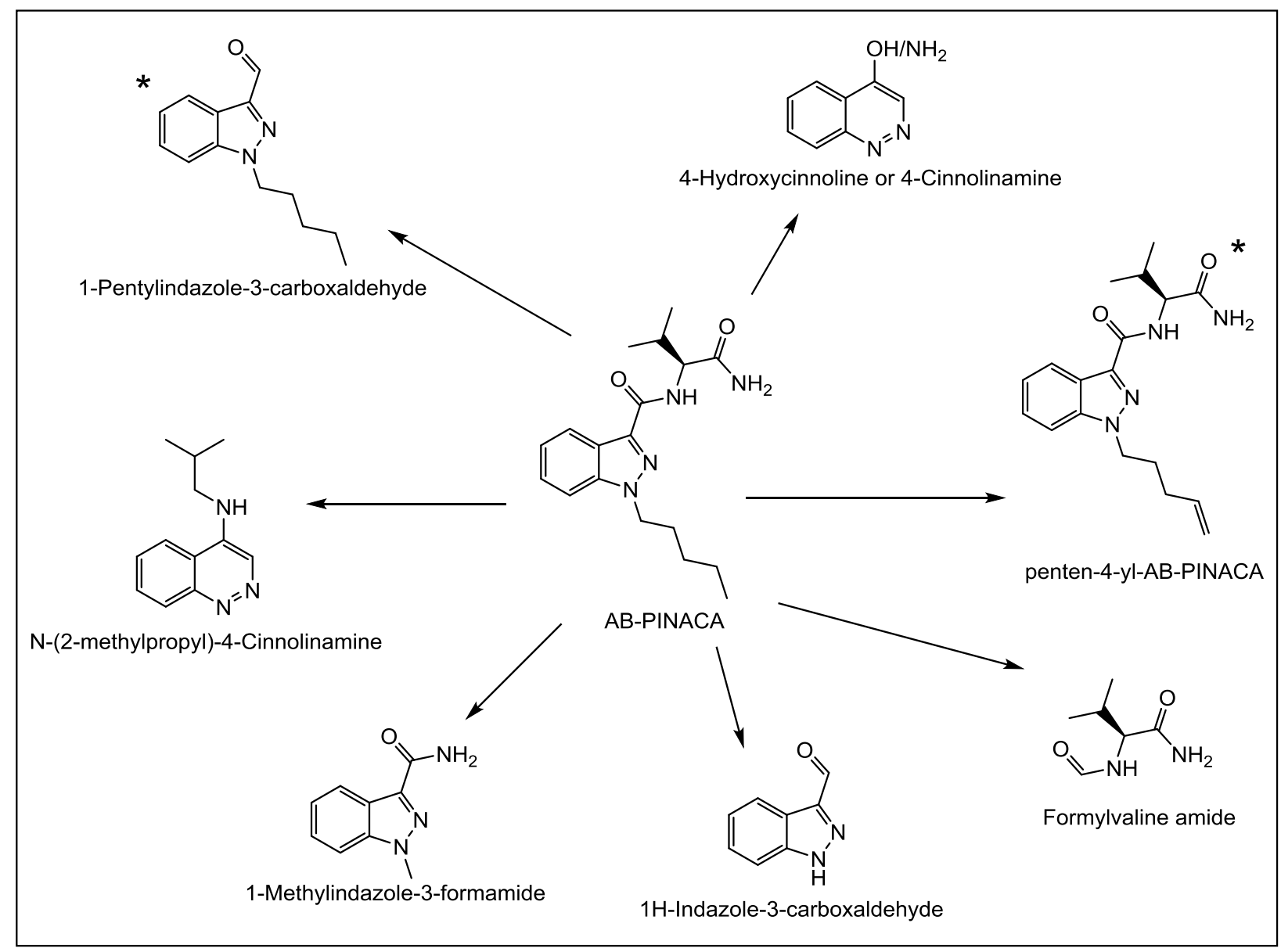

Figure C9 - Thermal degradation breakdown of AB-PINACA to its proposed observed pyrolytic products 


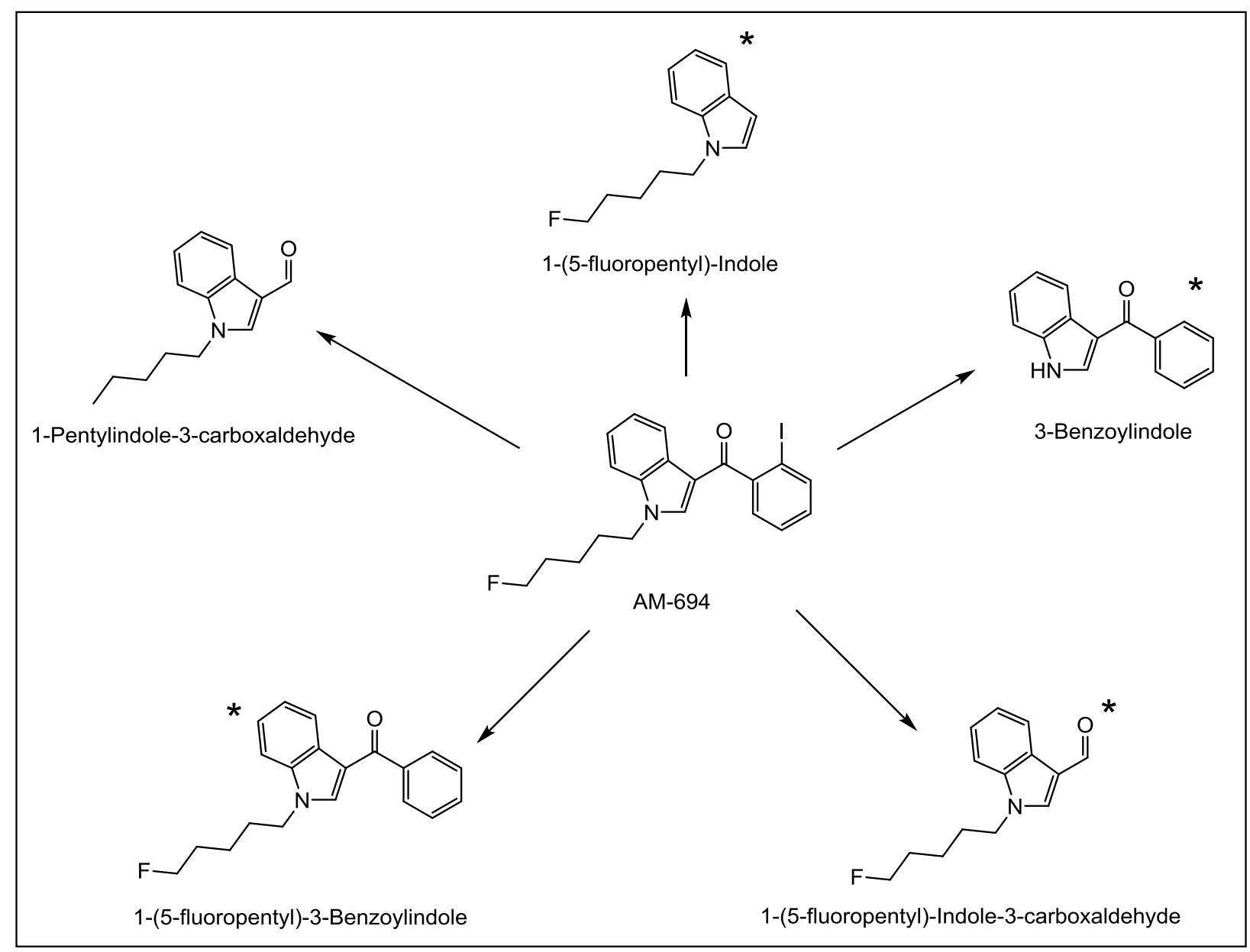

Figure C10 - Thermal degradation breakdown of AM-694 to its proposed observed pyrolytic products 


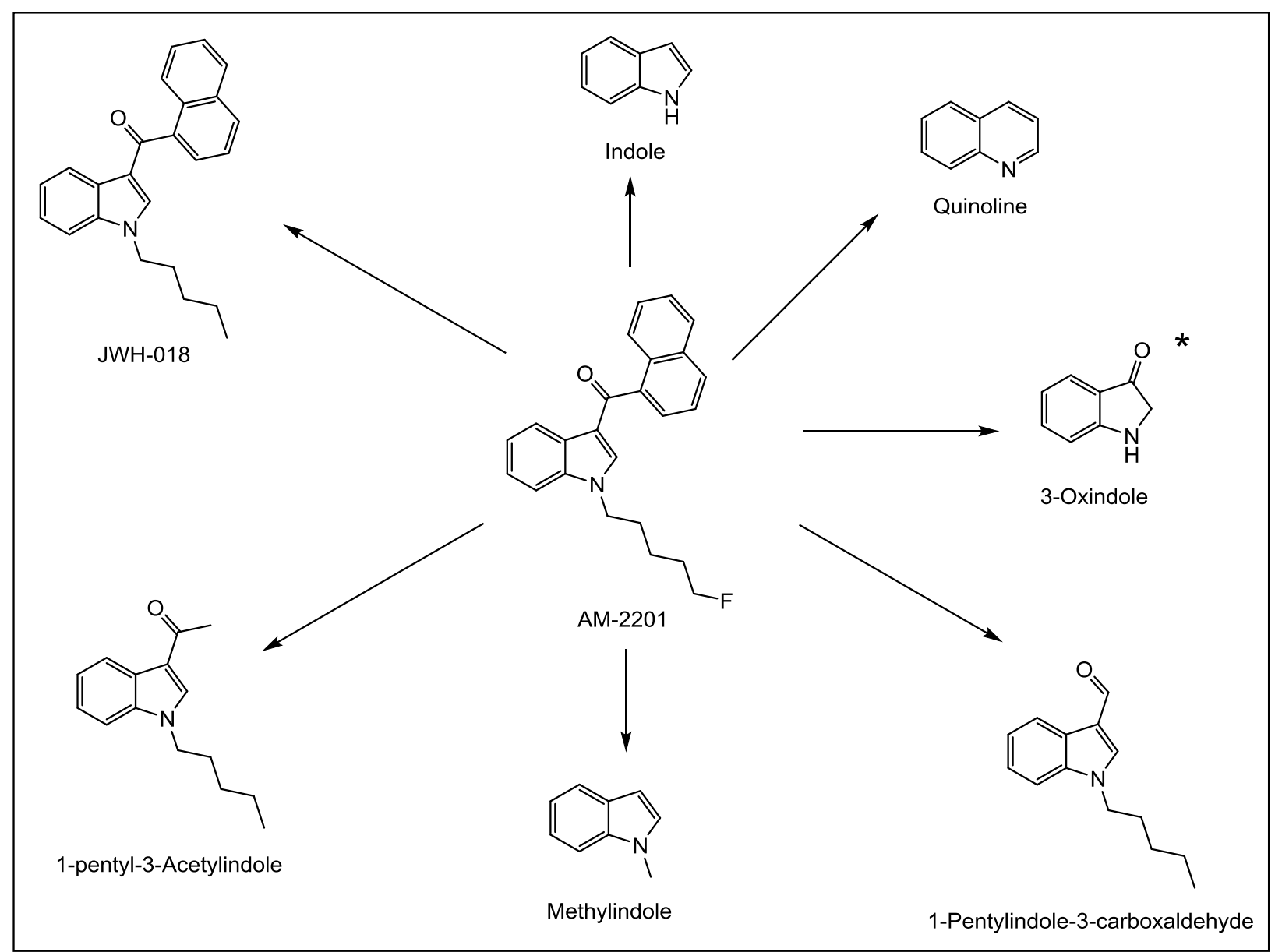

Figure C11 - Thermal degradation breakdown of AM-2201 to its proposed observed pyrolytic products 


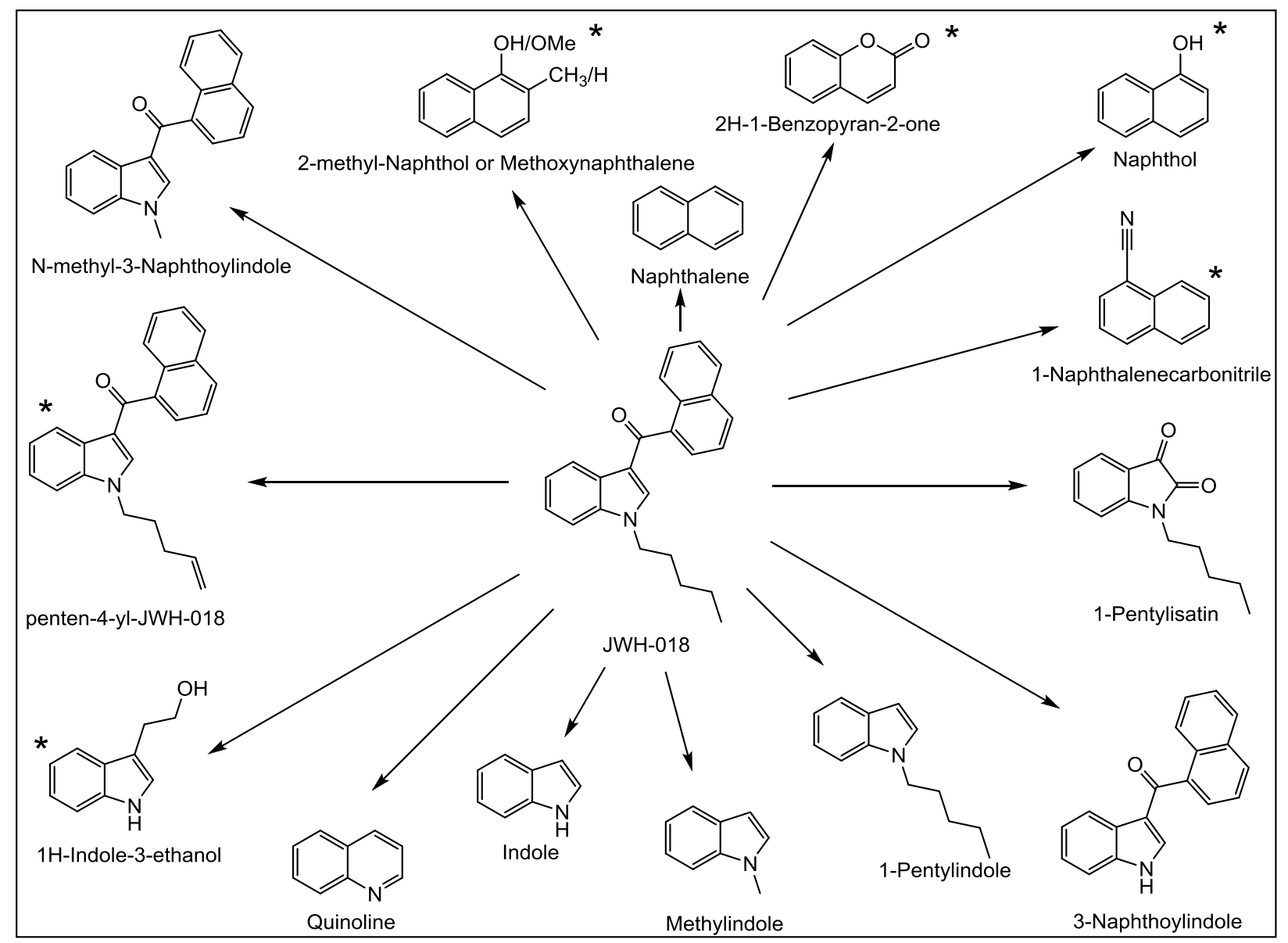

Figure C12 - Thermal degradation breakdown of JWH-018 to its proposed observed pyrolytic products 


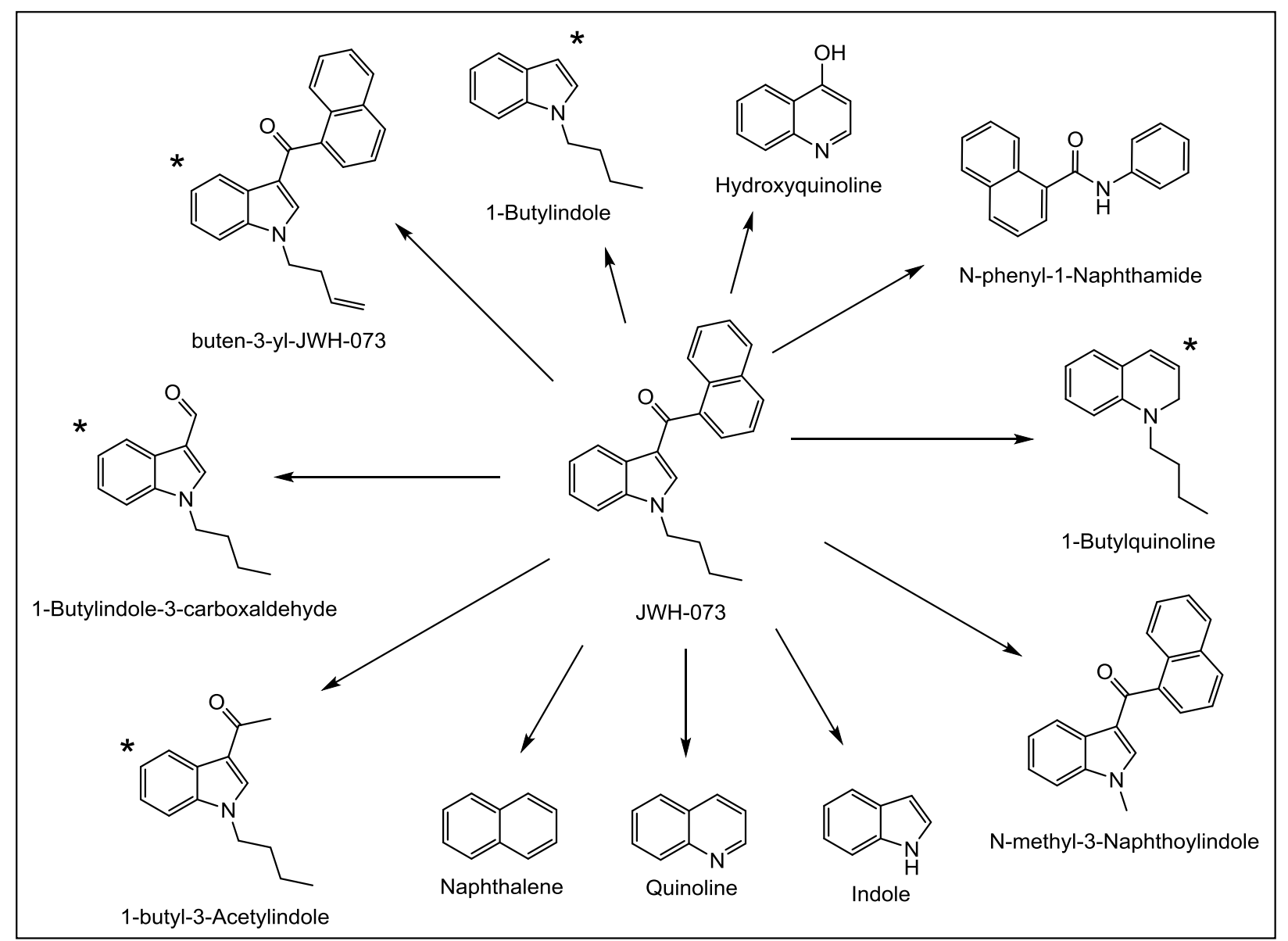

Figure C13 - Thermal degradation breakdown of JWH-073 to its proposed observed pyrolytic products 


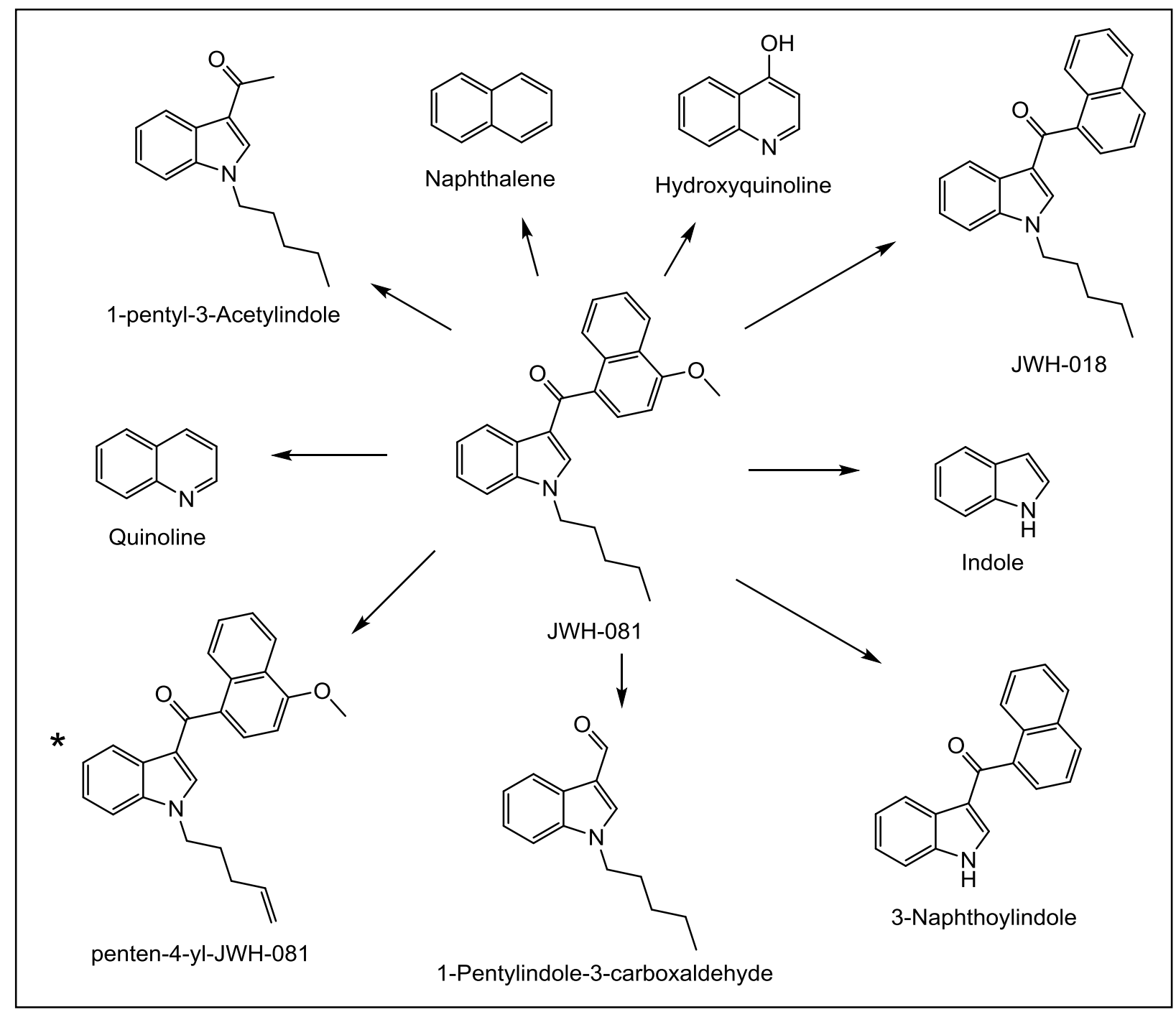

Figure C14 - Thermal degradation breakdown of JWH-081 to its proposed observed pyrolytic products 


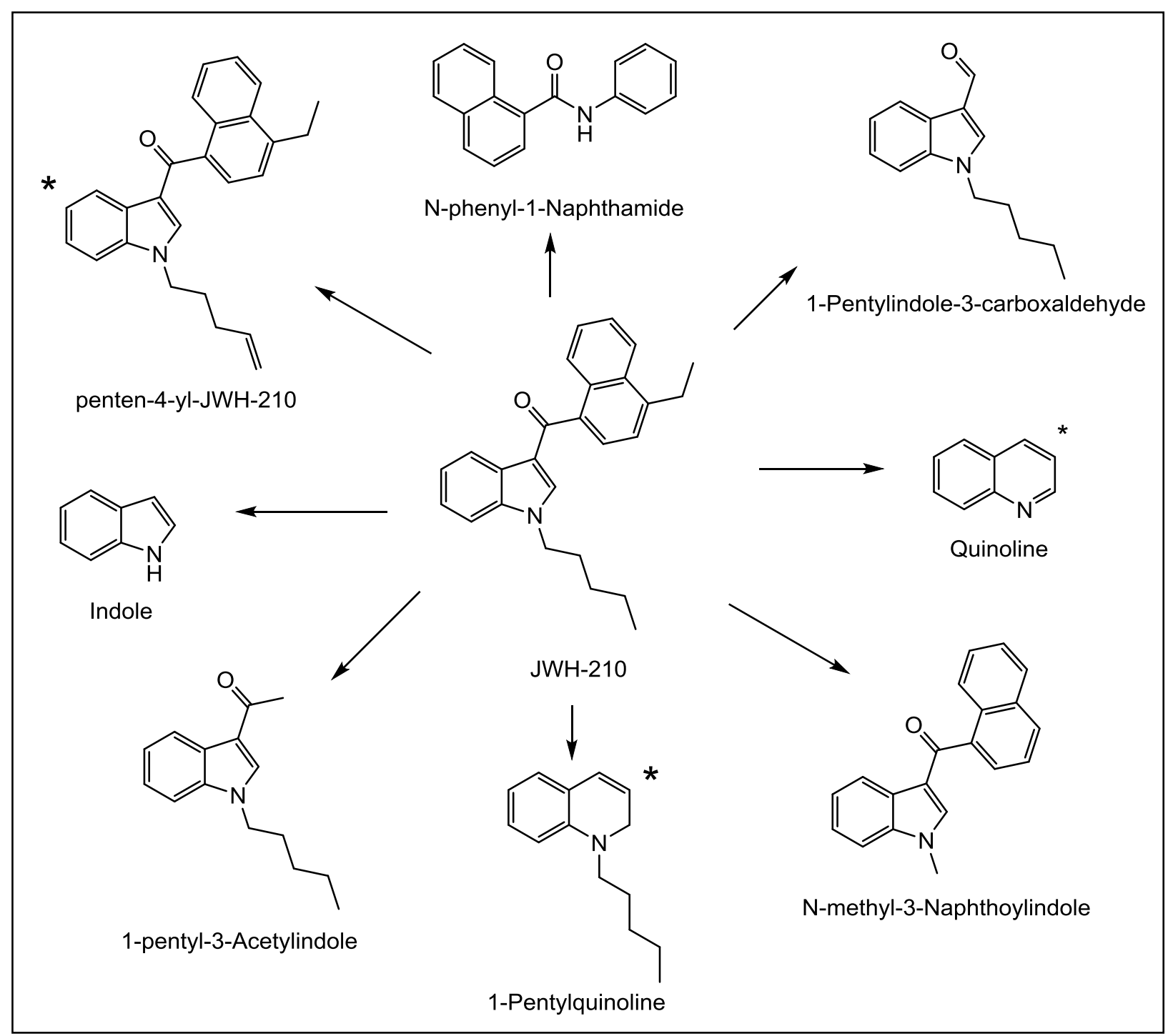

Figure C15 - Thermal degradation breakdown of JWH-210 to its proposed observed pyrolytic products 


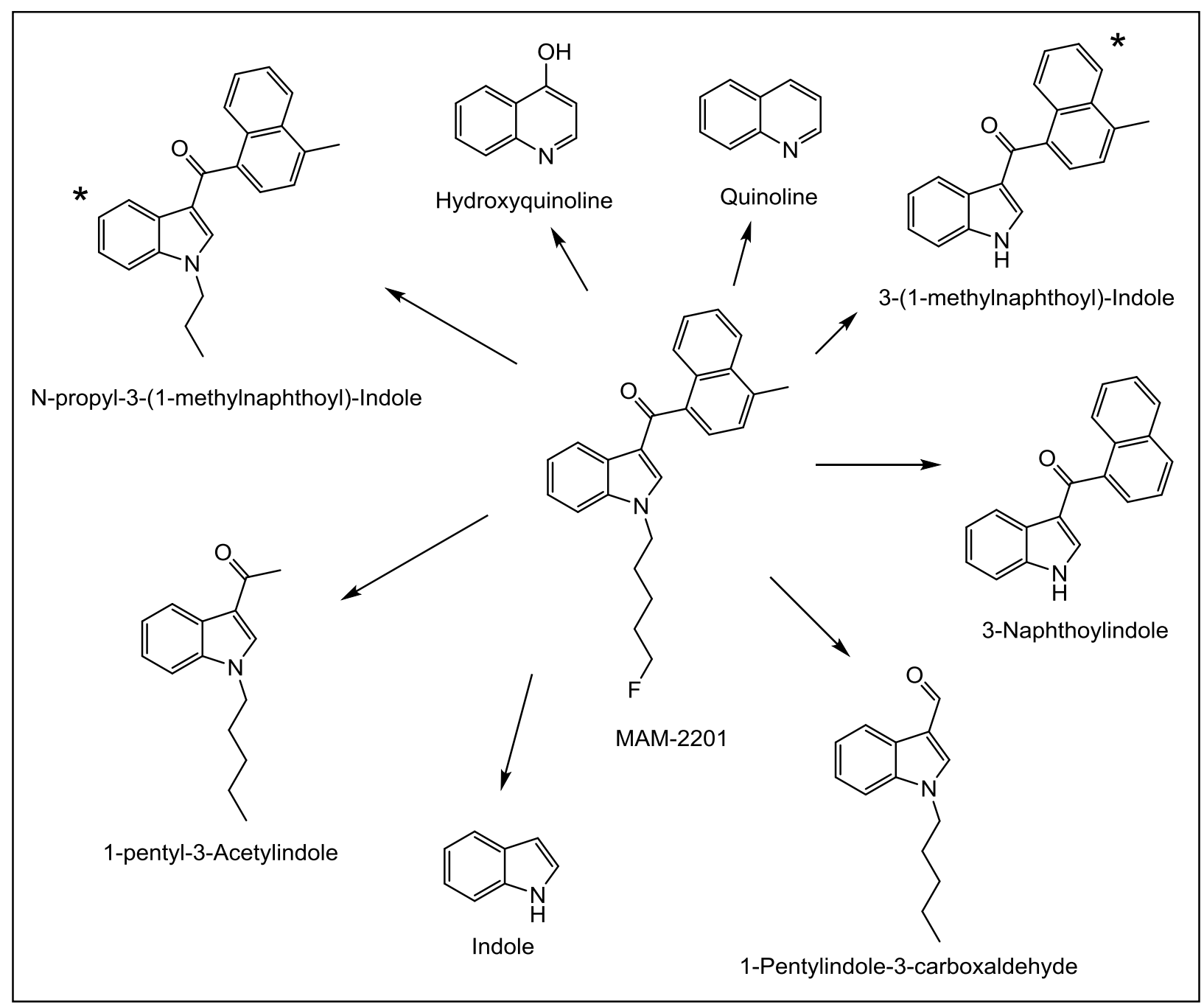

Figure C16 - Thermal degradation breakdown of MAM-2201 to its proposed observed pyrolytic products 


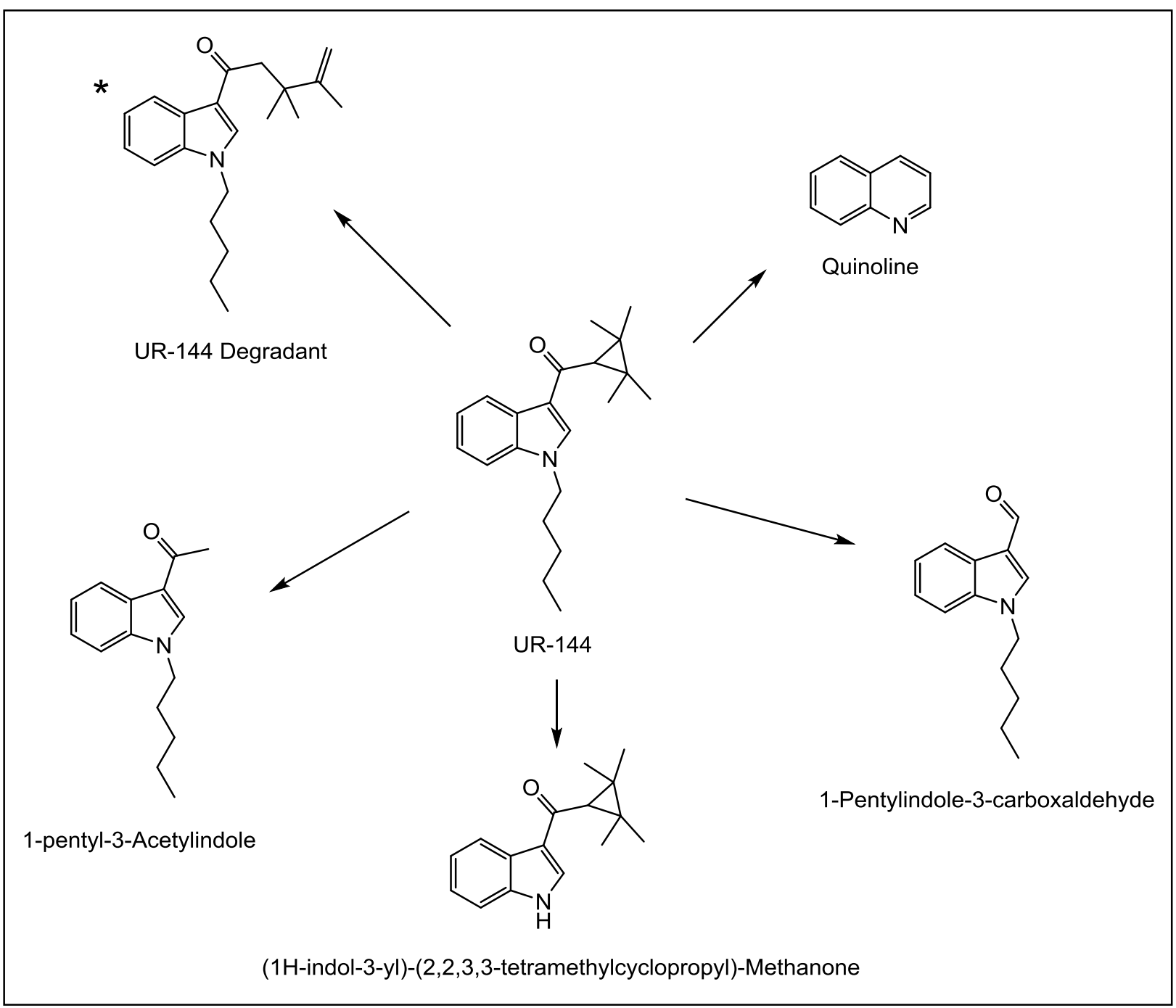

Figure C17 - Thermal degradation breakdown of UR-144 to its proposed observed pyrolytic products 


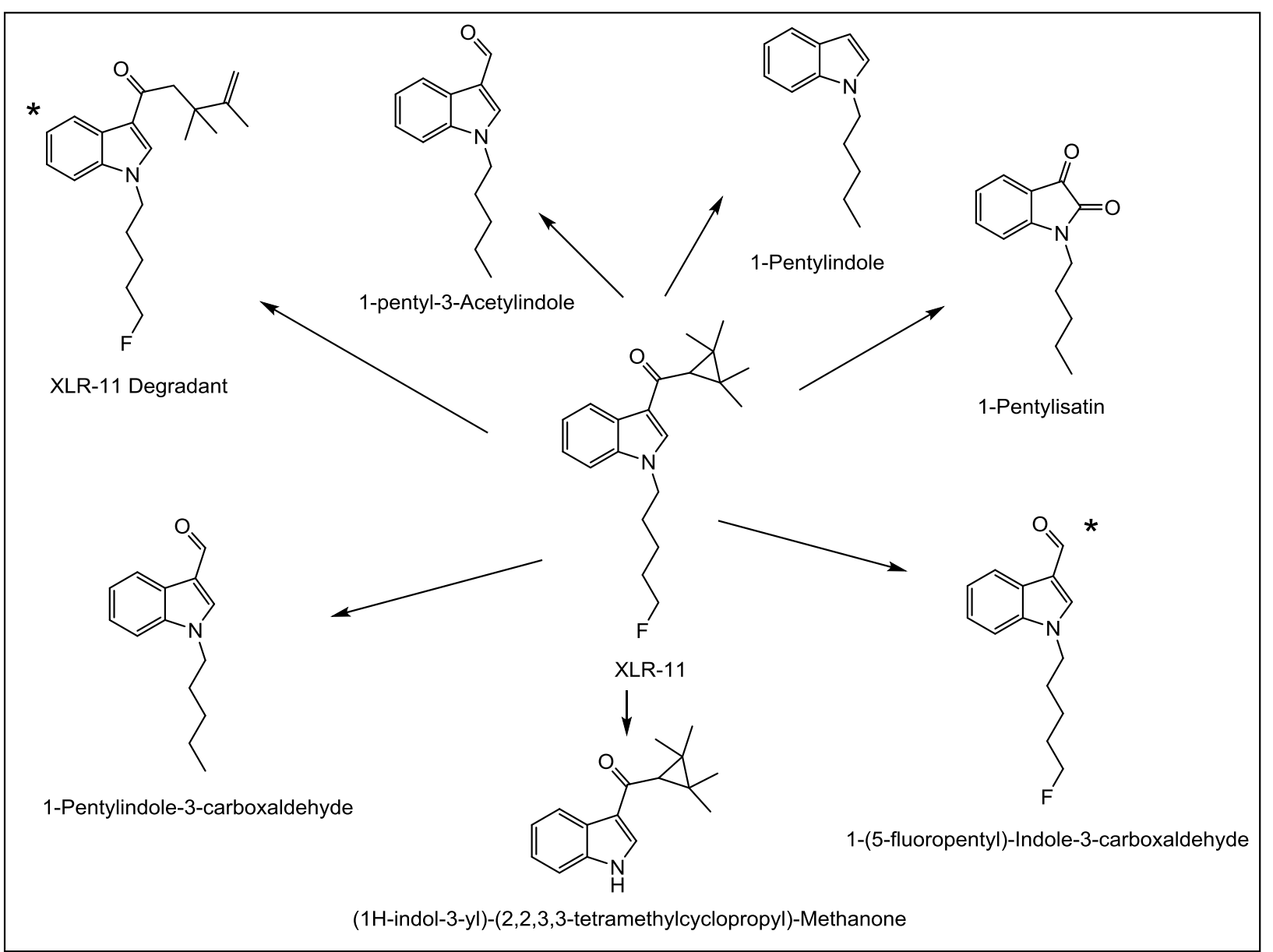

Figure C18 - Thermal degradation breakdown of XLR-11 to its proposed observed pyrolytic products 
Shared Proposed Pyrolytic Products from Parent Synthetic Cannabinoid Breakdowns:

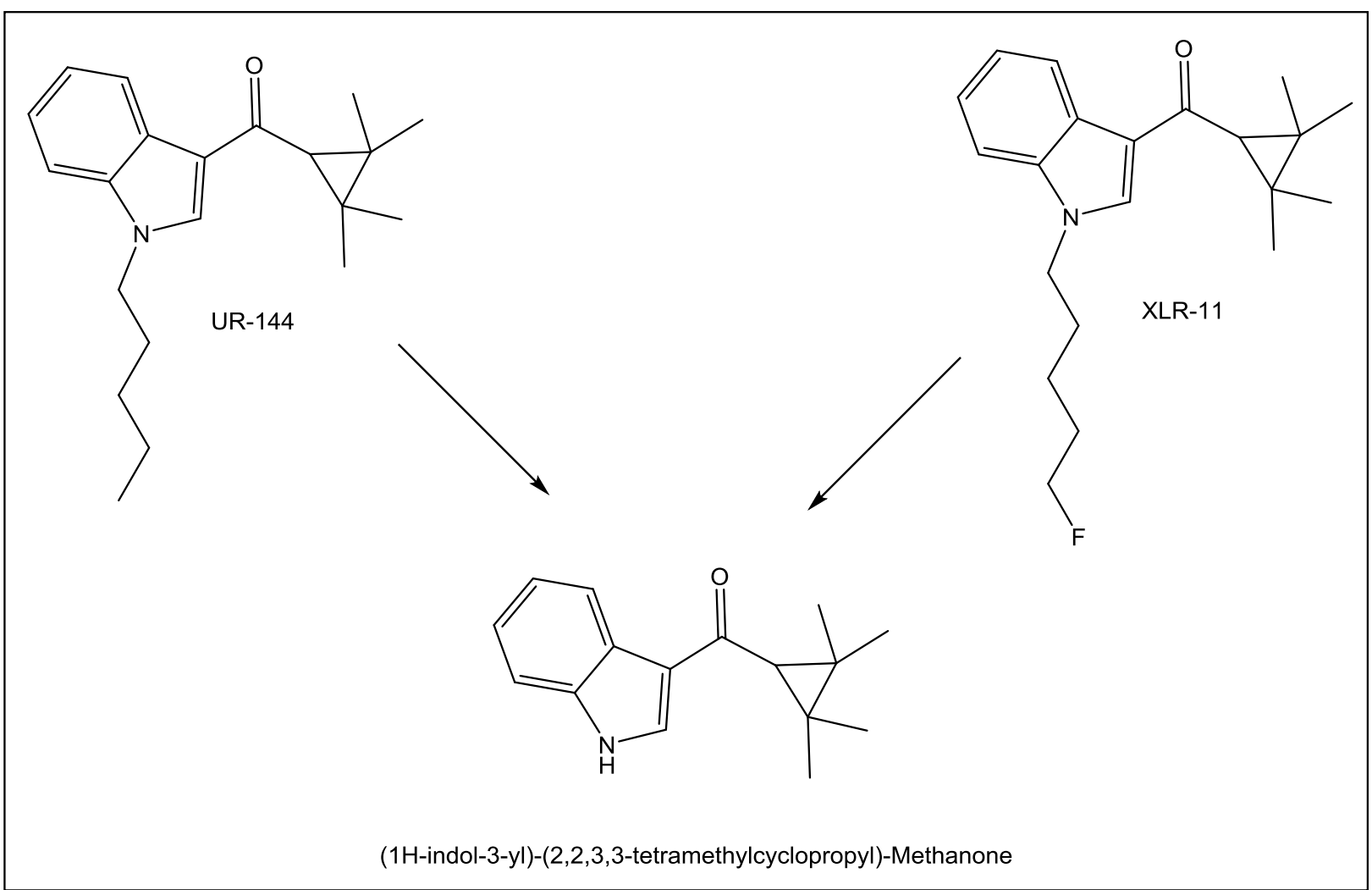

Figure C19 - Breakdown of parent synthetic cannabinoids that produced the proposed thermal degradant of (1H-indol-3-yl)-(2,2,3,3-tetramethylcyclopropyl)-Methanone 


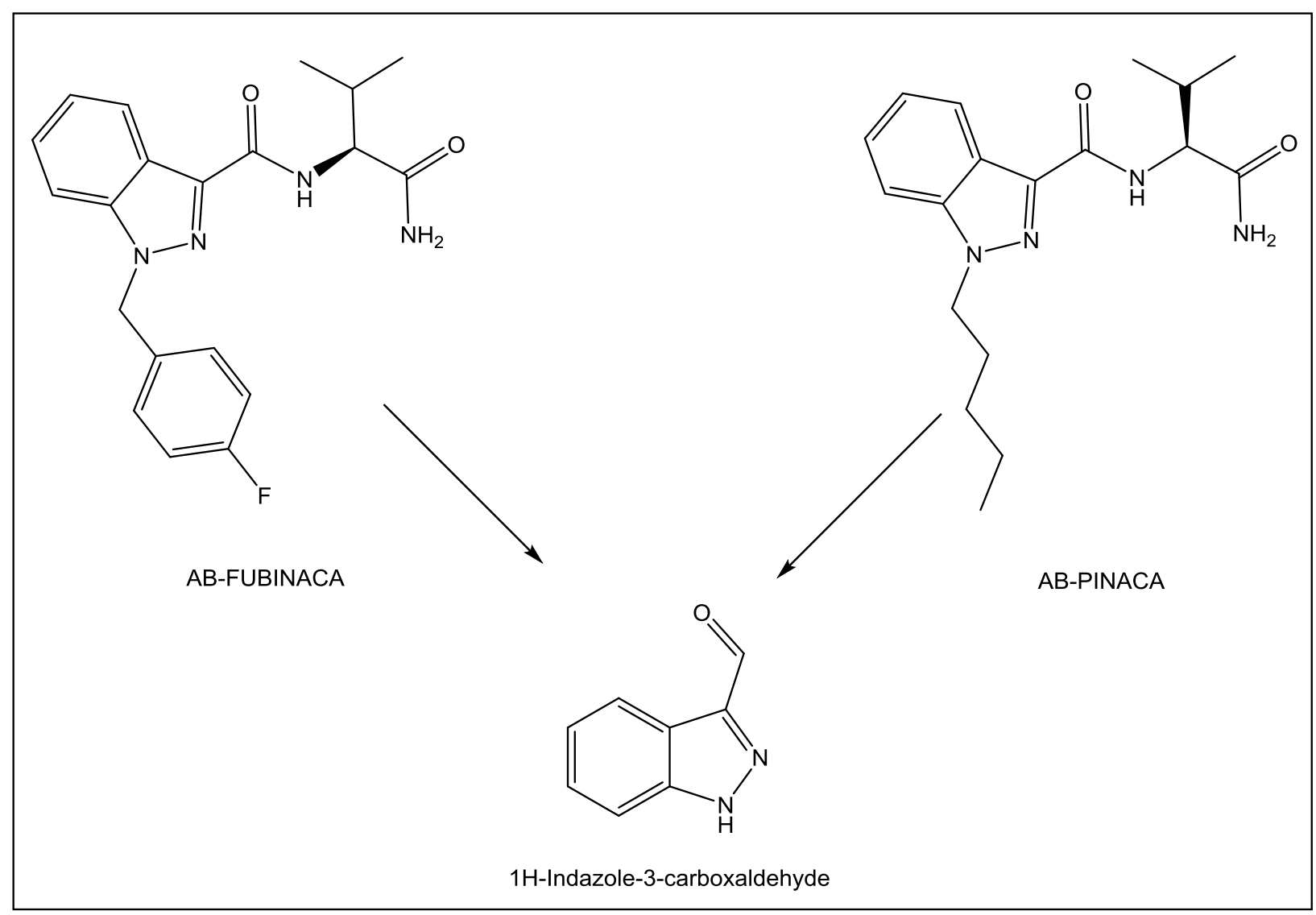

Figure C20 - Breakdown of parent synthetic cannabinoids that produced the proposed thermal degradant of 1H-Indazole-3-carboxaldehyde 


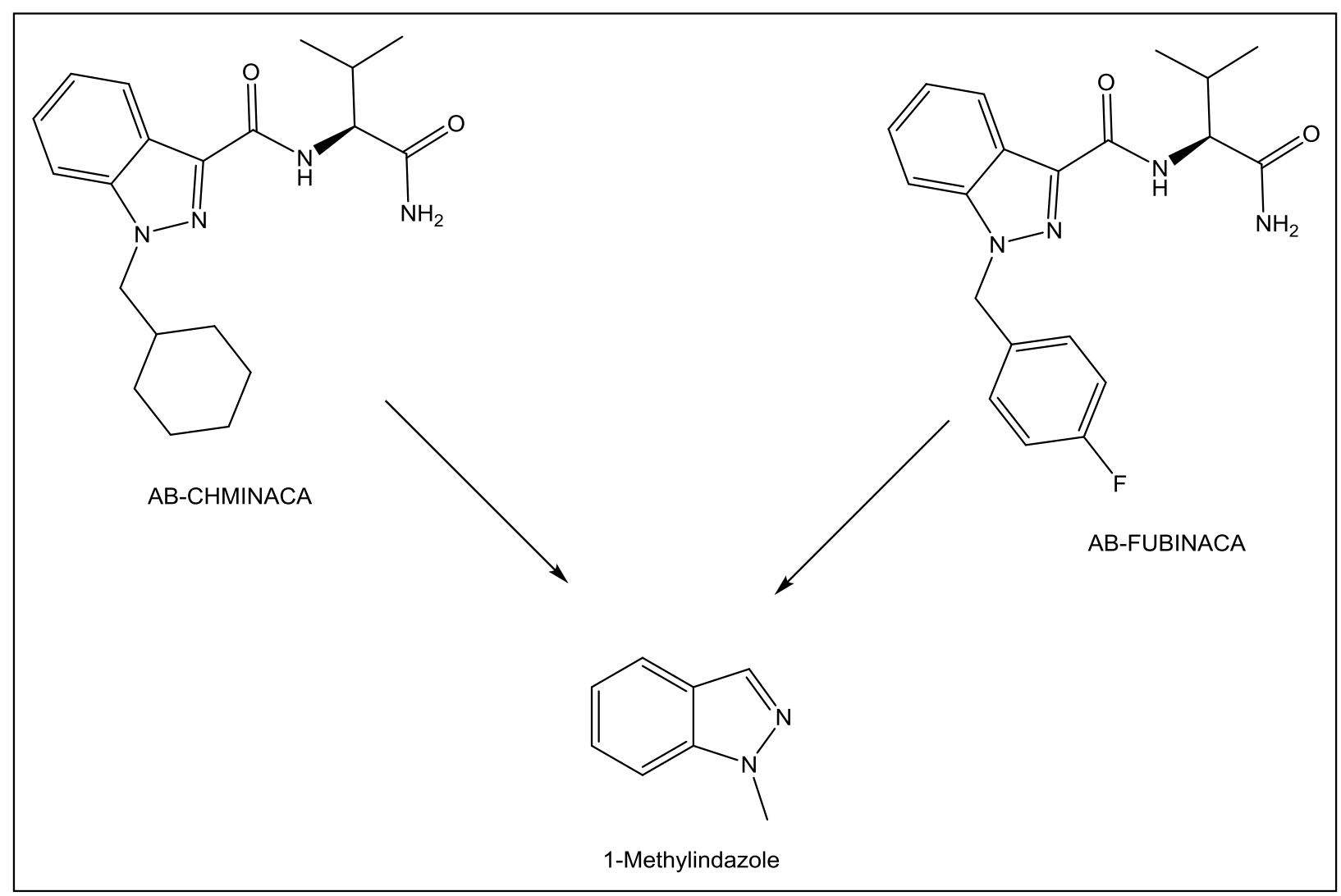

Figure C21 - Breakdown of parent synthetic cannabinoids that produced the proposed thermal degradant of 1-Methylindazole 


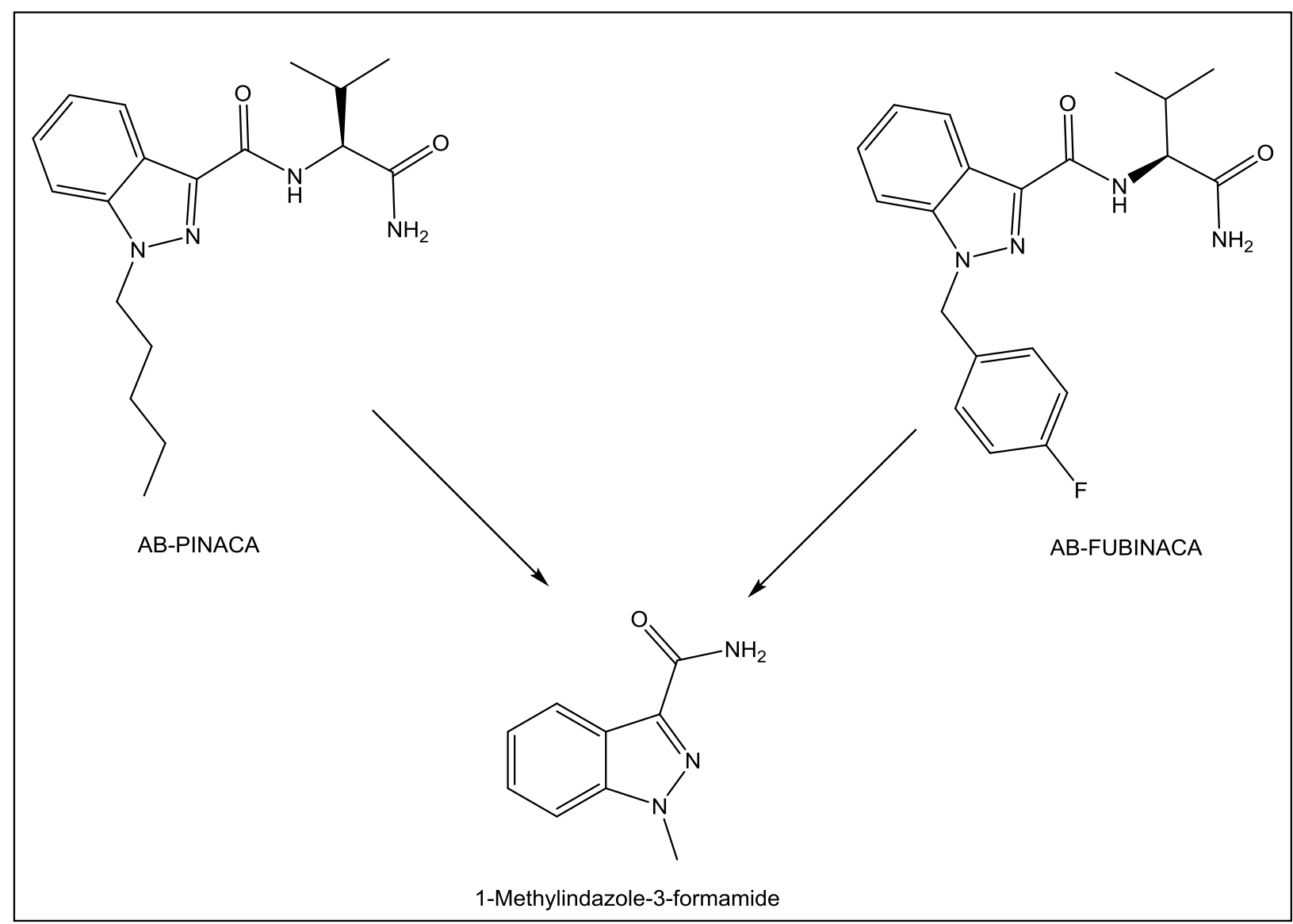

Figure C22 - Breakdown of parent synthetic cannabinoids that produced the proposed thermal degradant of 1-Methylindazole-3-formamide 


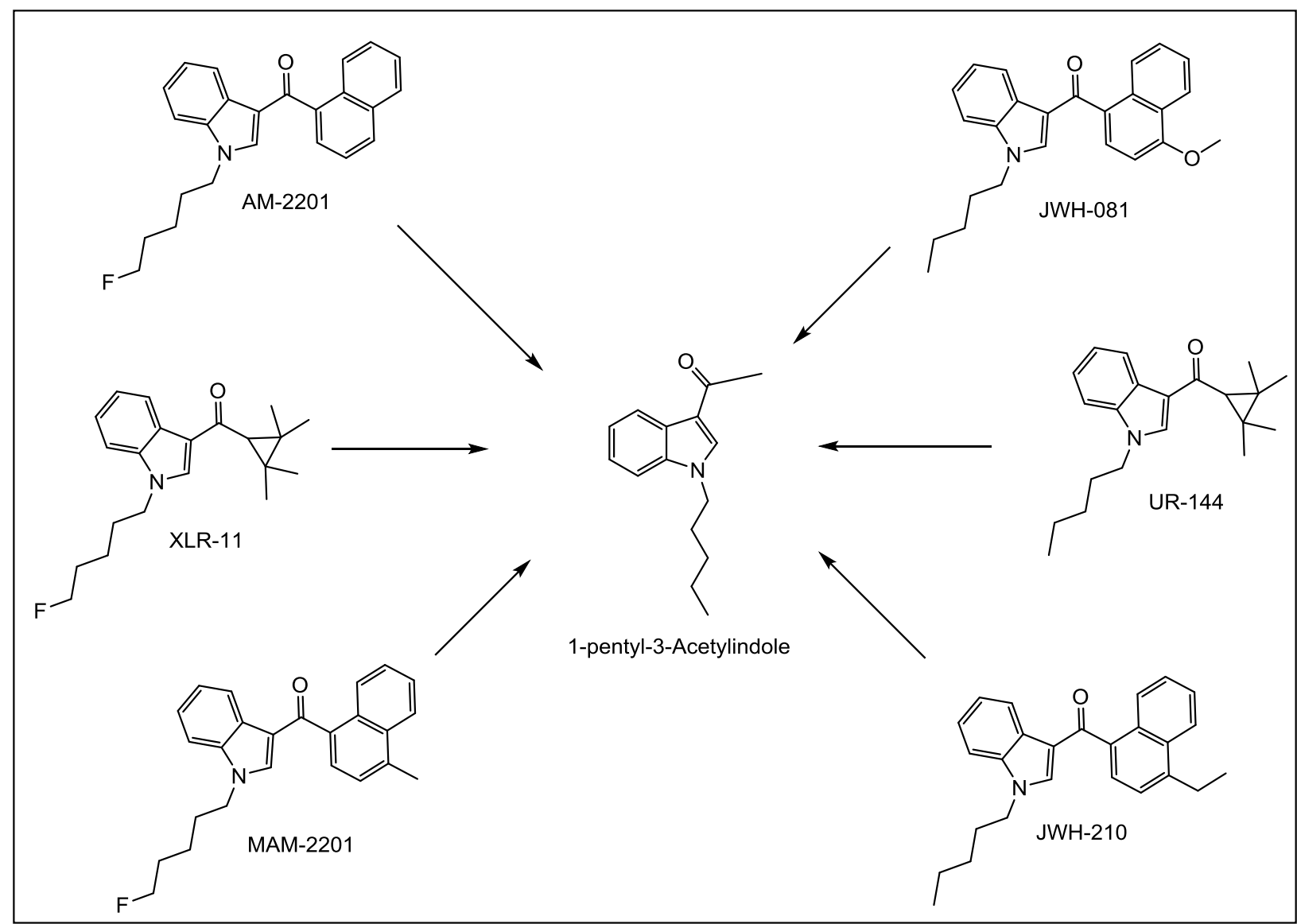

Figure C23 - Breakdown of parent synthetic cannabinoids that produced the proposed thermal degradant of 1-pentyl-3-Acetylindole 


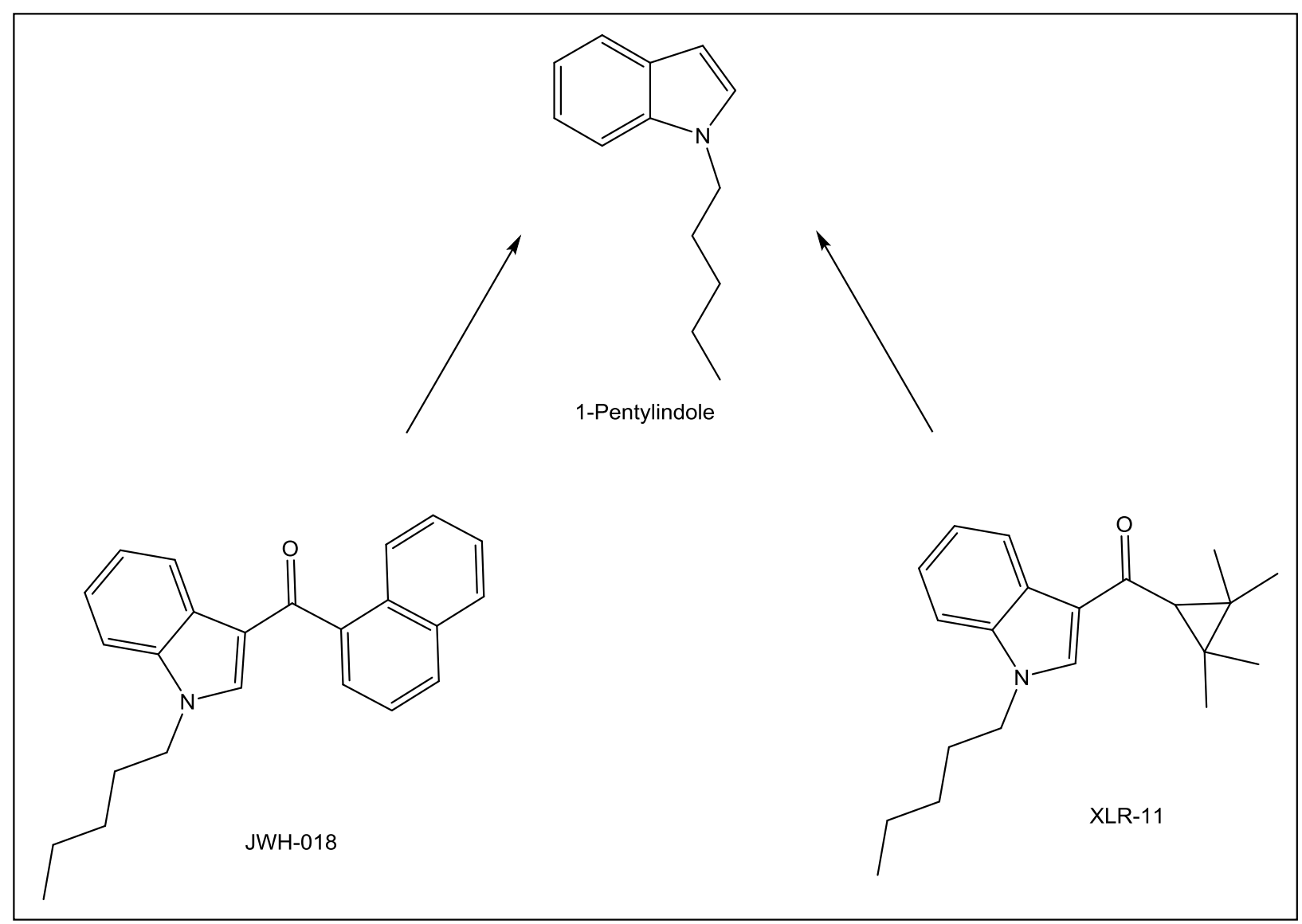

Figure C24 - Breakdown of parent synthetic cannabinoids that produced the proposed thermal degradant of 1-Pentylindole 


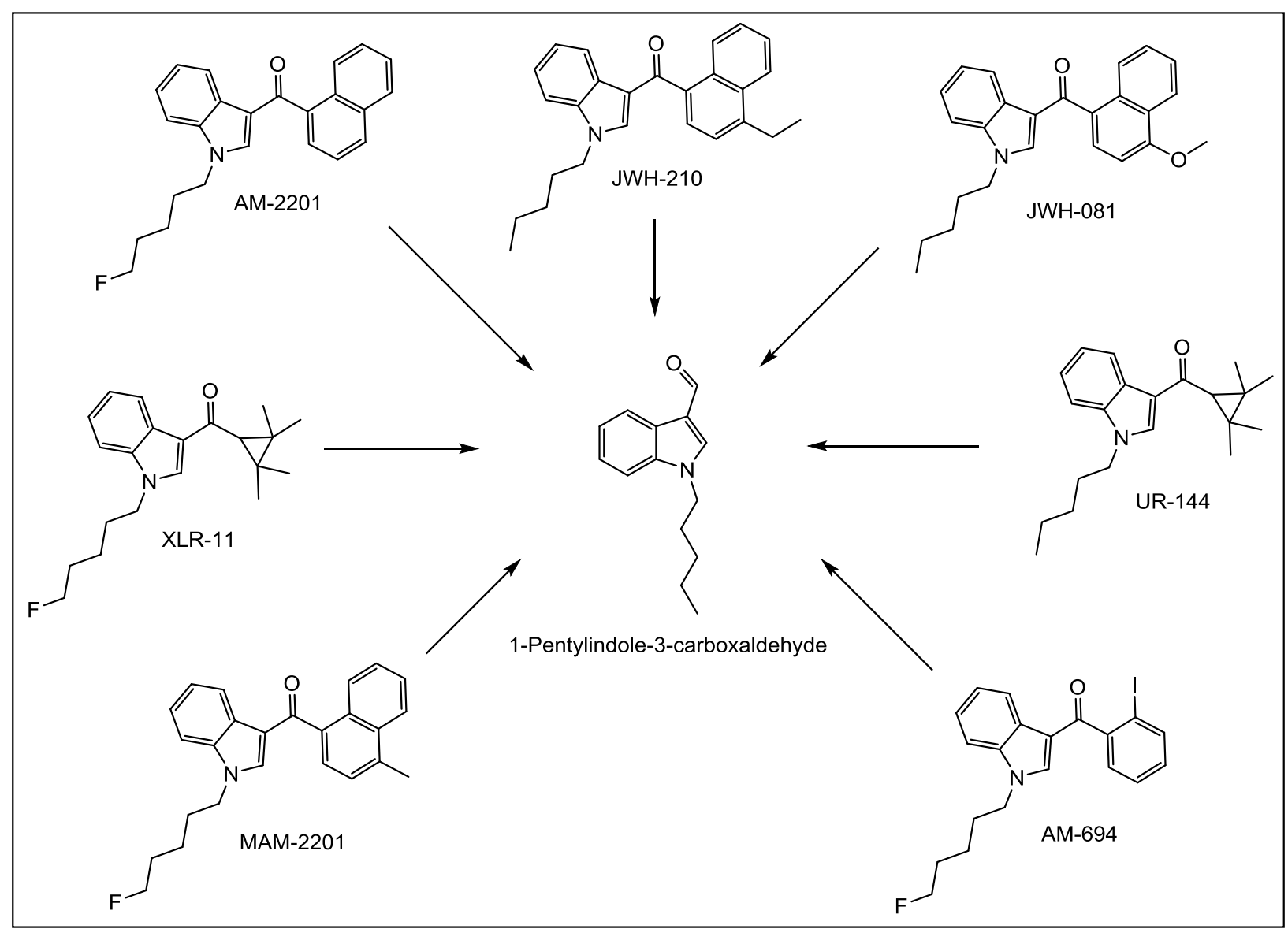

Figure C25 - Breakdown of parent synthetic cannabinoids that produced the proposed thermal degradant of 1-Pentylindole-3-carboxaldehyde 


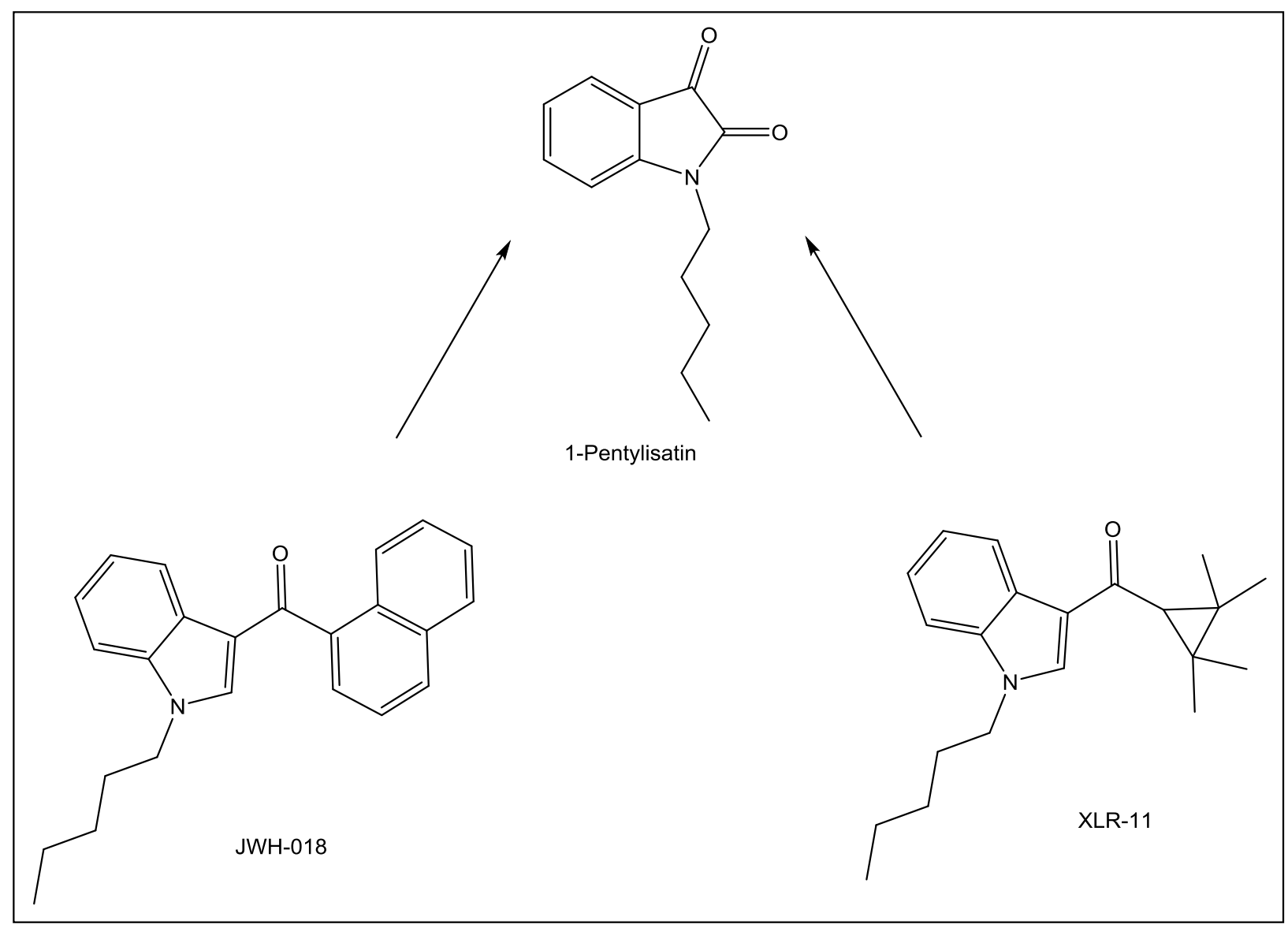

Figure C26 - Breakdown of parent synthetic cannabinoids that produced the proposed thermal degradant of 1-Pentylisatin 


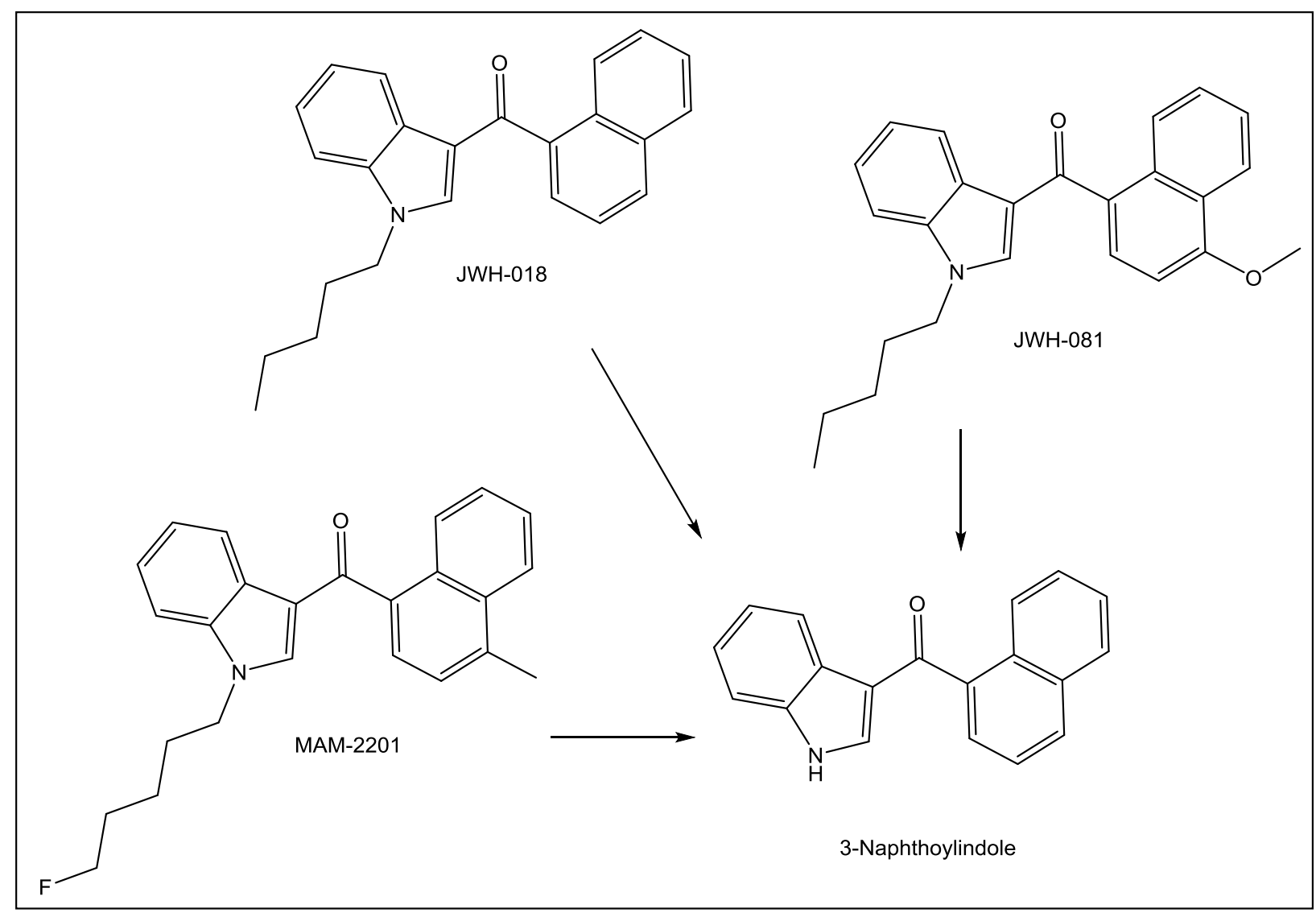

Figure C27 - Breakdown of parent synthetic cannabinoids that produced the proposed thermal degradant of 3-Naphthoylindole 


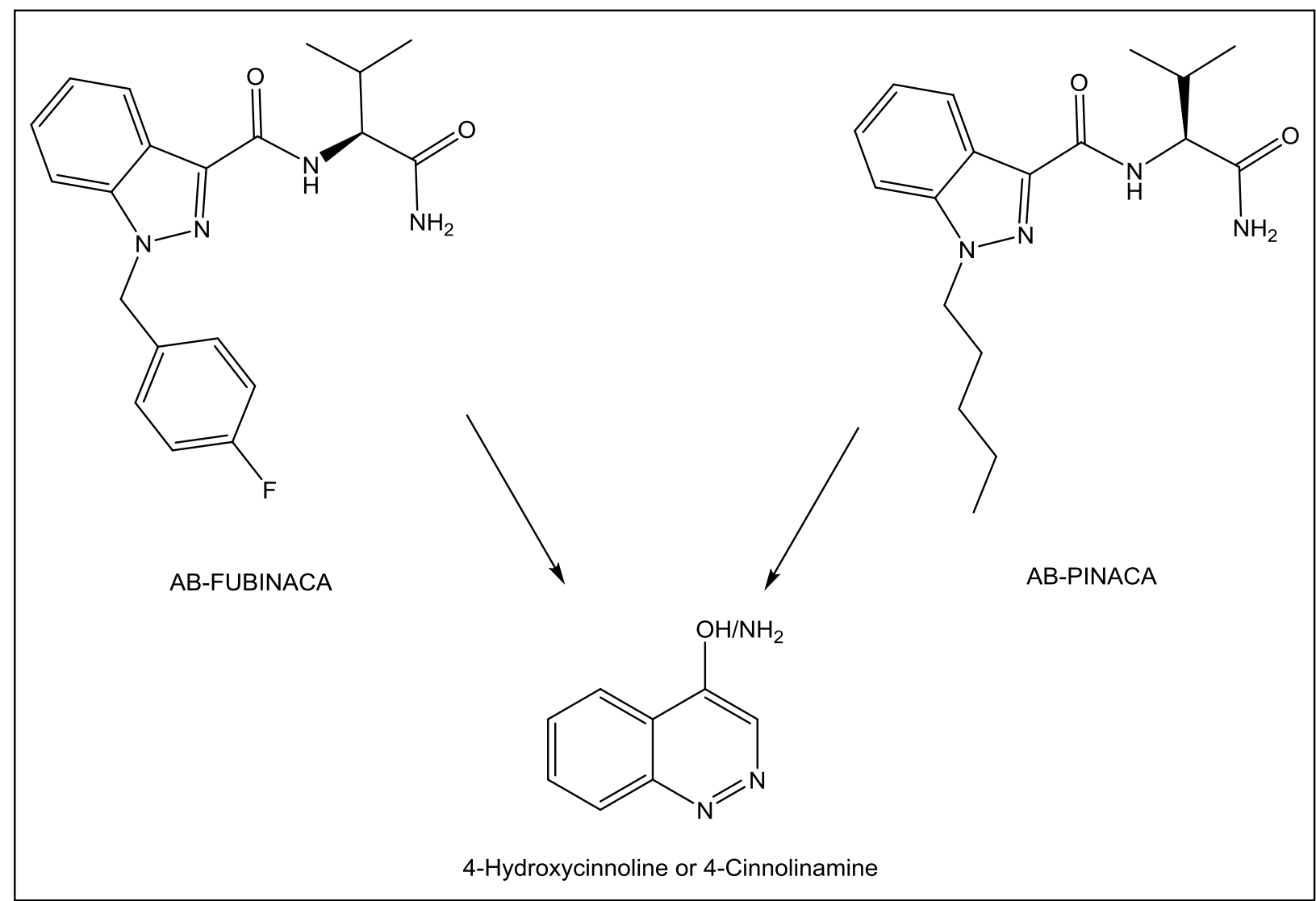

Figure C28 - Breakdown of parent synthetic cannabinoids that produced the proposed thermal degradant of 4-Hydroxycinnoline or 4-Cinnolinamine 


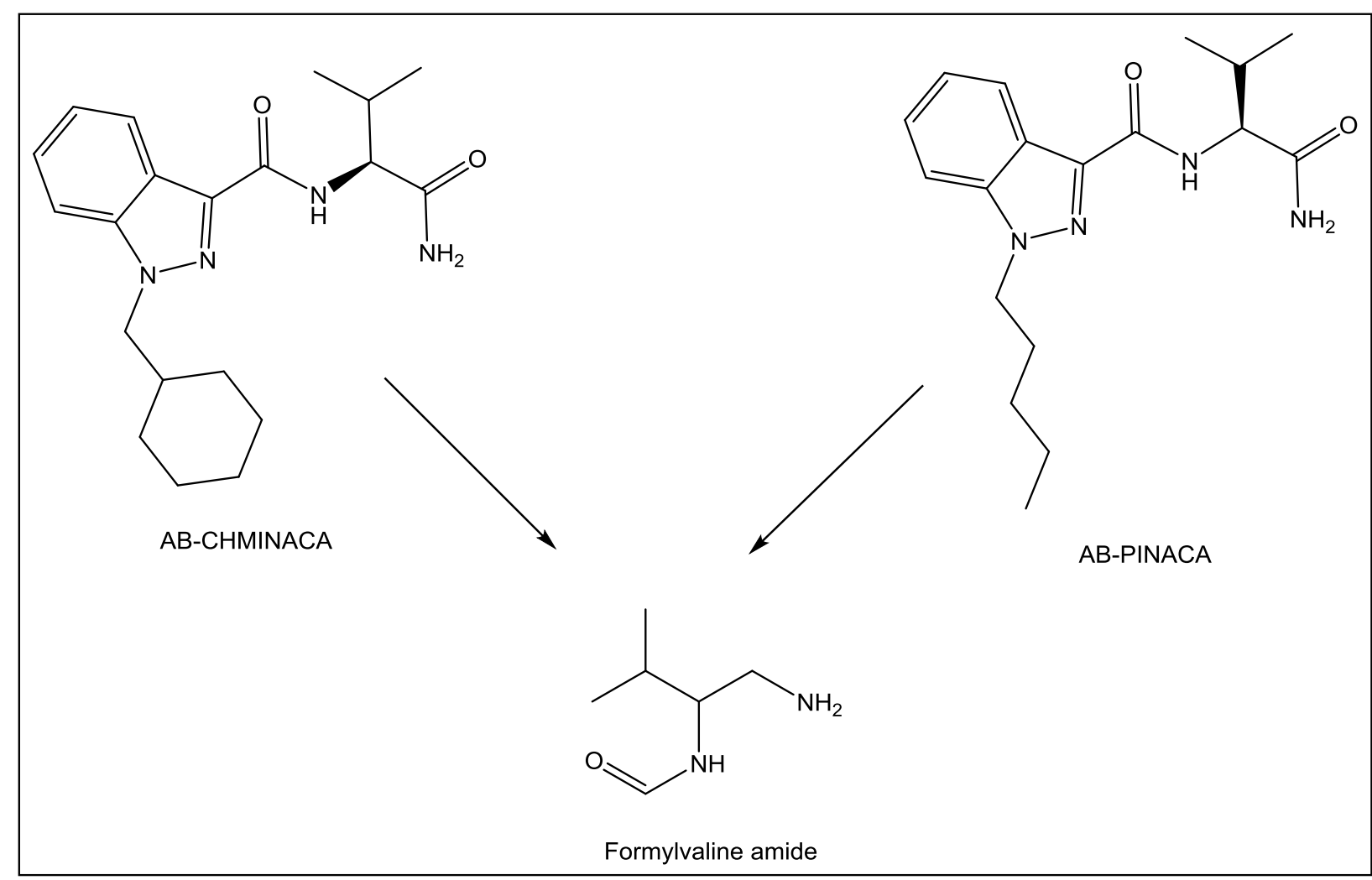

Figure C29 - Breakdown of parent synthetic cannabinoids that produced the proposed thermal degradant of Formylvaline amide 


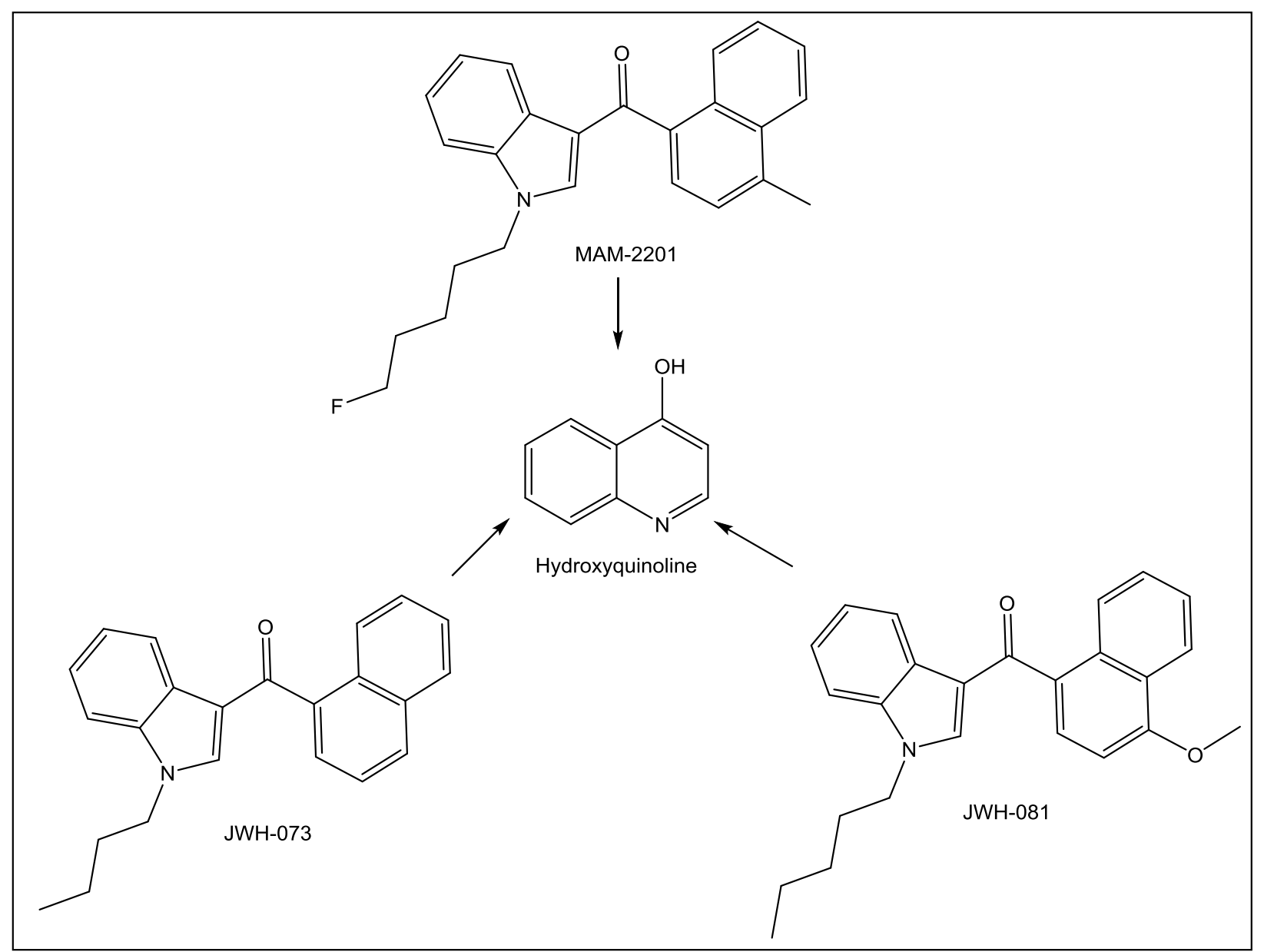

Figure C30 - Breakdown of parent synthetic cannabinoids that produced the proposed thermal degradant of Hydroxyquinoline 


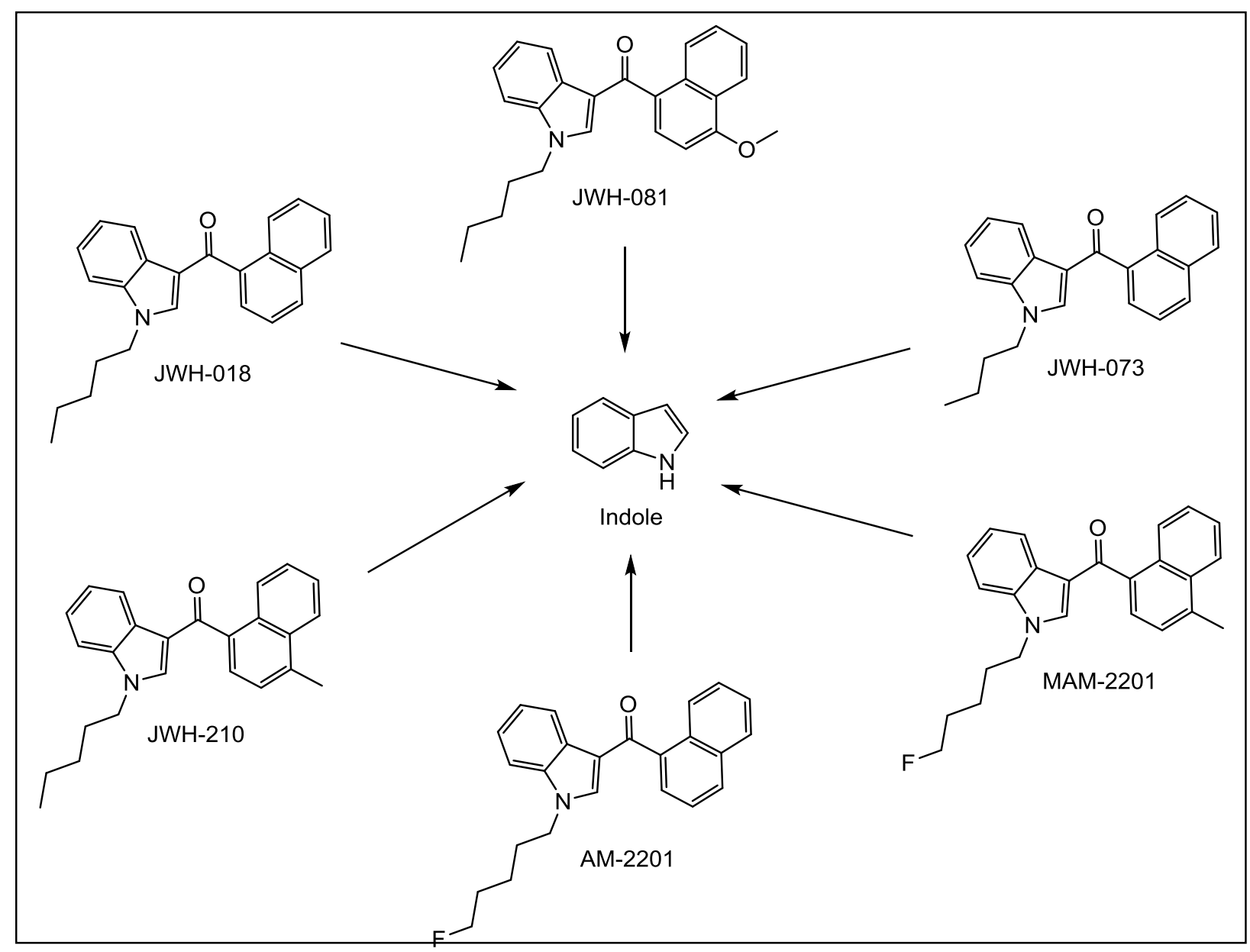

Figure C31 - Breakdown of parent synthetic cannabinoids that produced the proposed thermal degradant of Indole 


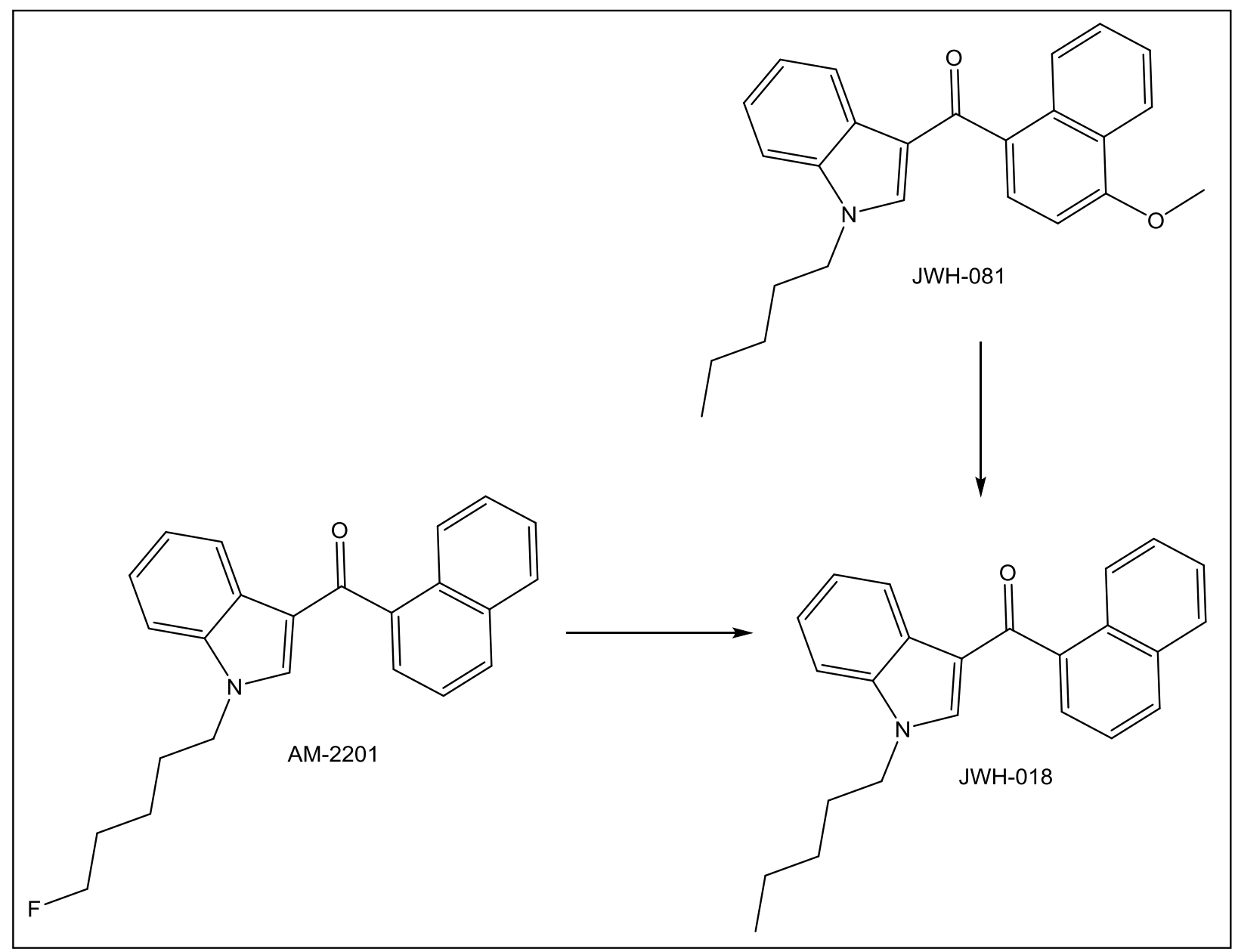

Figure C32 - Breakdown of parent synthetic cannabinoids that produced the proposed thermal degradant of JWH-018 


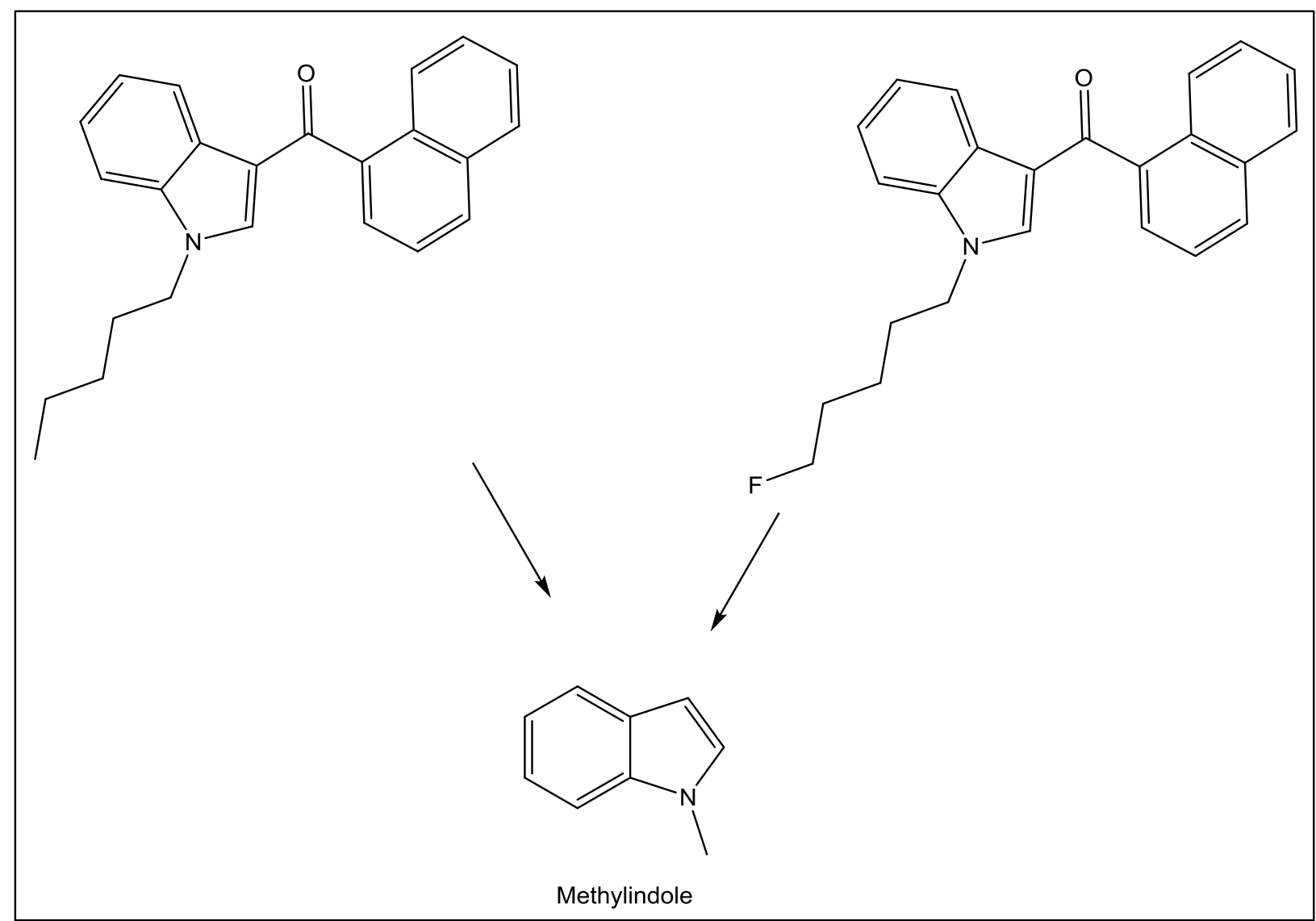

Figure C33 - Breakdown of parent synthetic cannabinoids that produced the proposed thermal degradant of Methylindole 


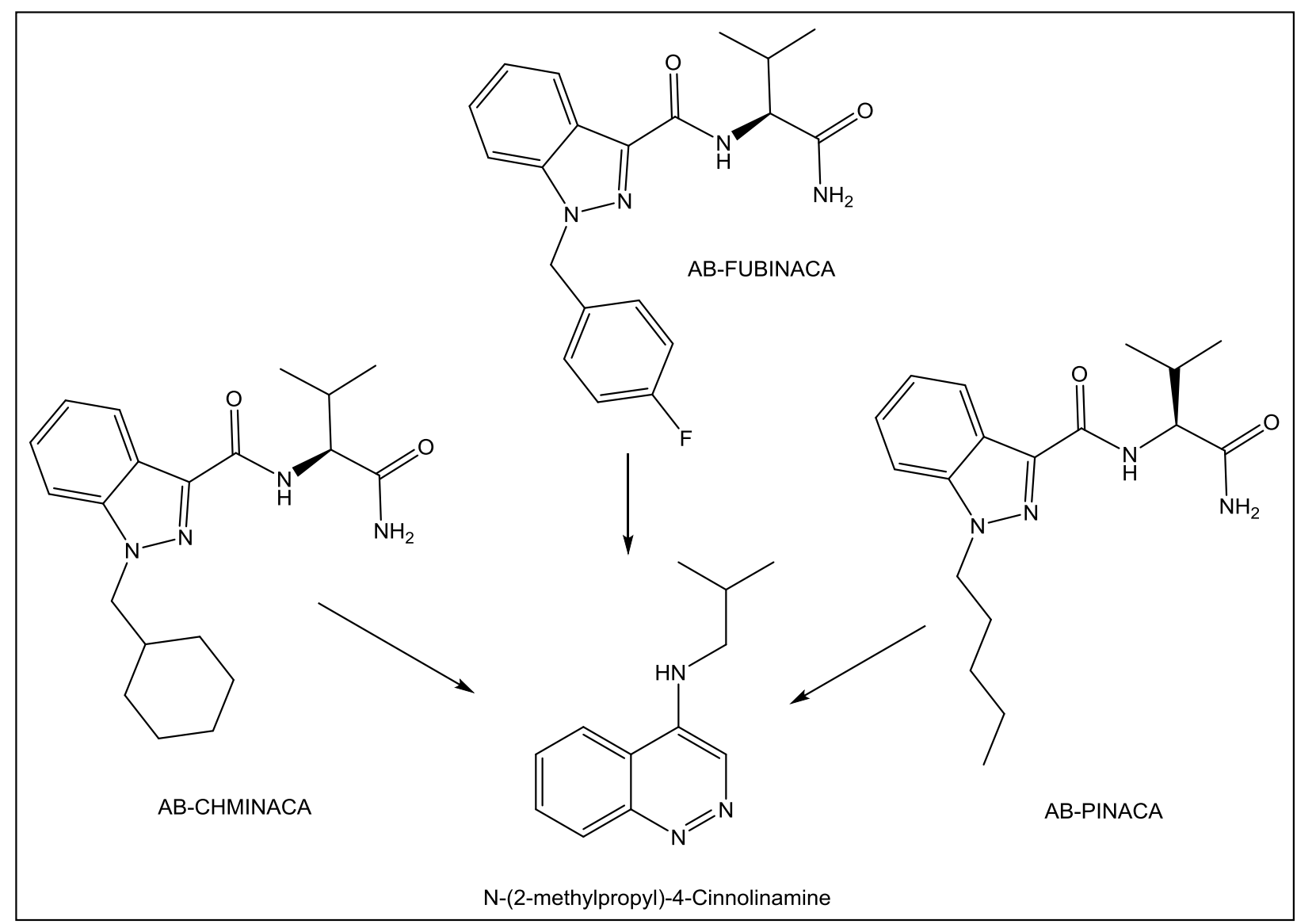

Figure C34 - Breakdown of parent synthetic cannabinoids that produced the proposed thermal degradant of N-(2-methylpropyl)-4-Cinnolinamine 


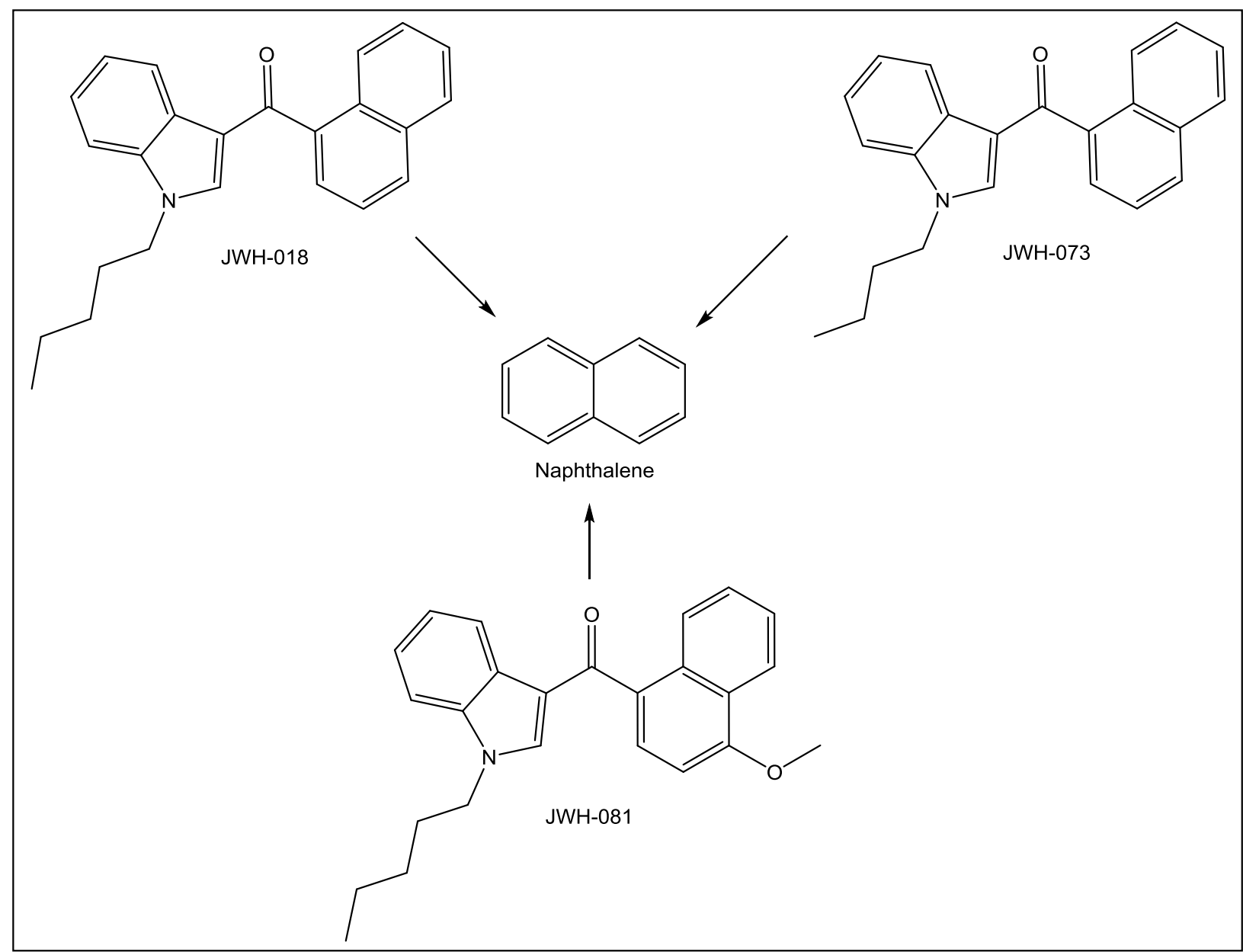

Figure C35 - Breakdown of parent synthetic cannabinoids that produced the proposed thermal degradant of Naphthalene 


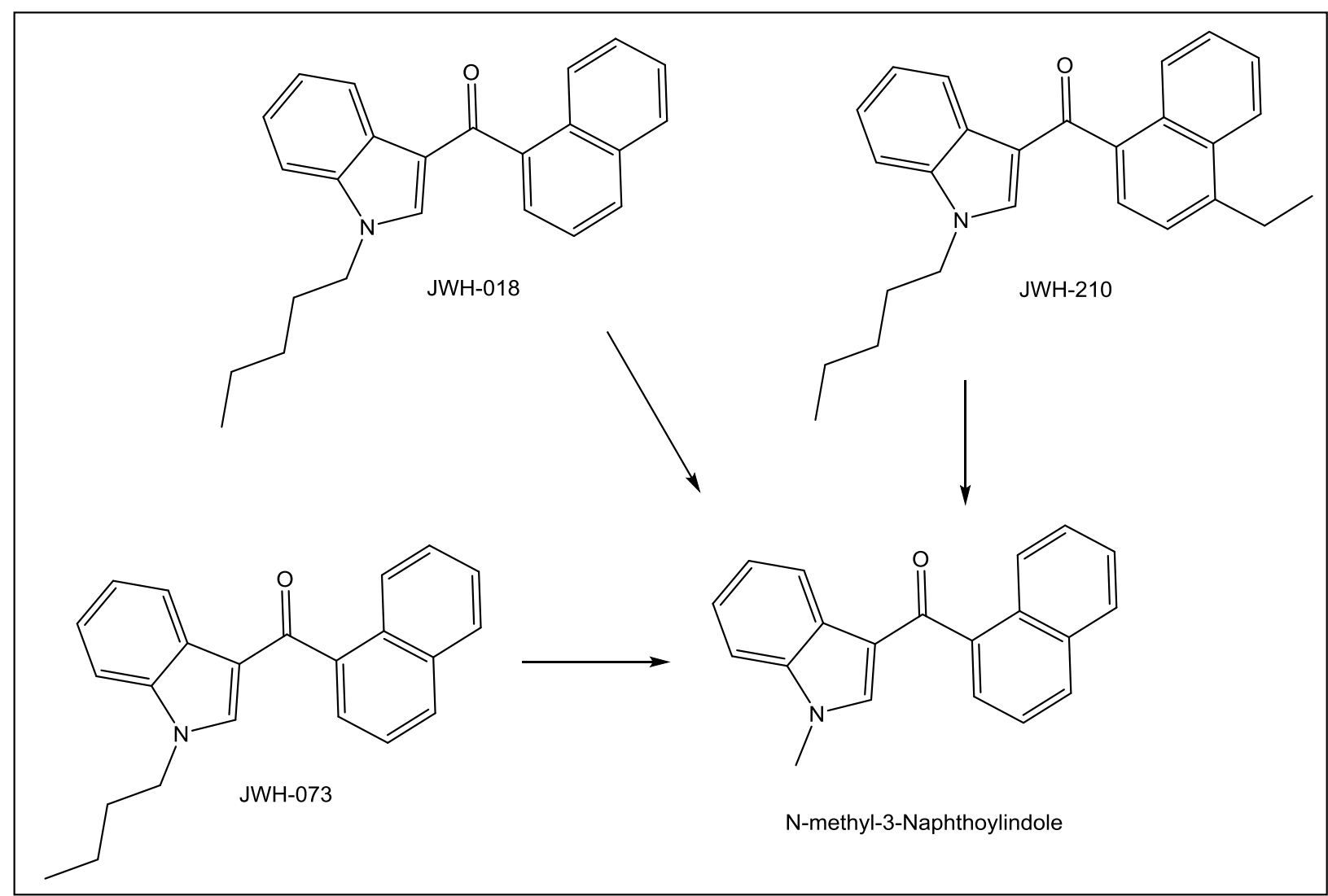

Figure C36 - Breakdown of parent synthetic cannabinoids that produced the proposed thermal degradant of N-methyl-3-Naphthoylindole 


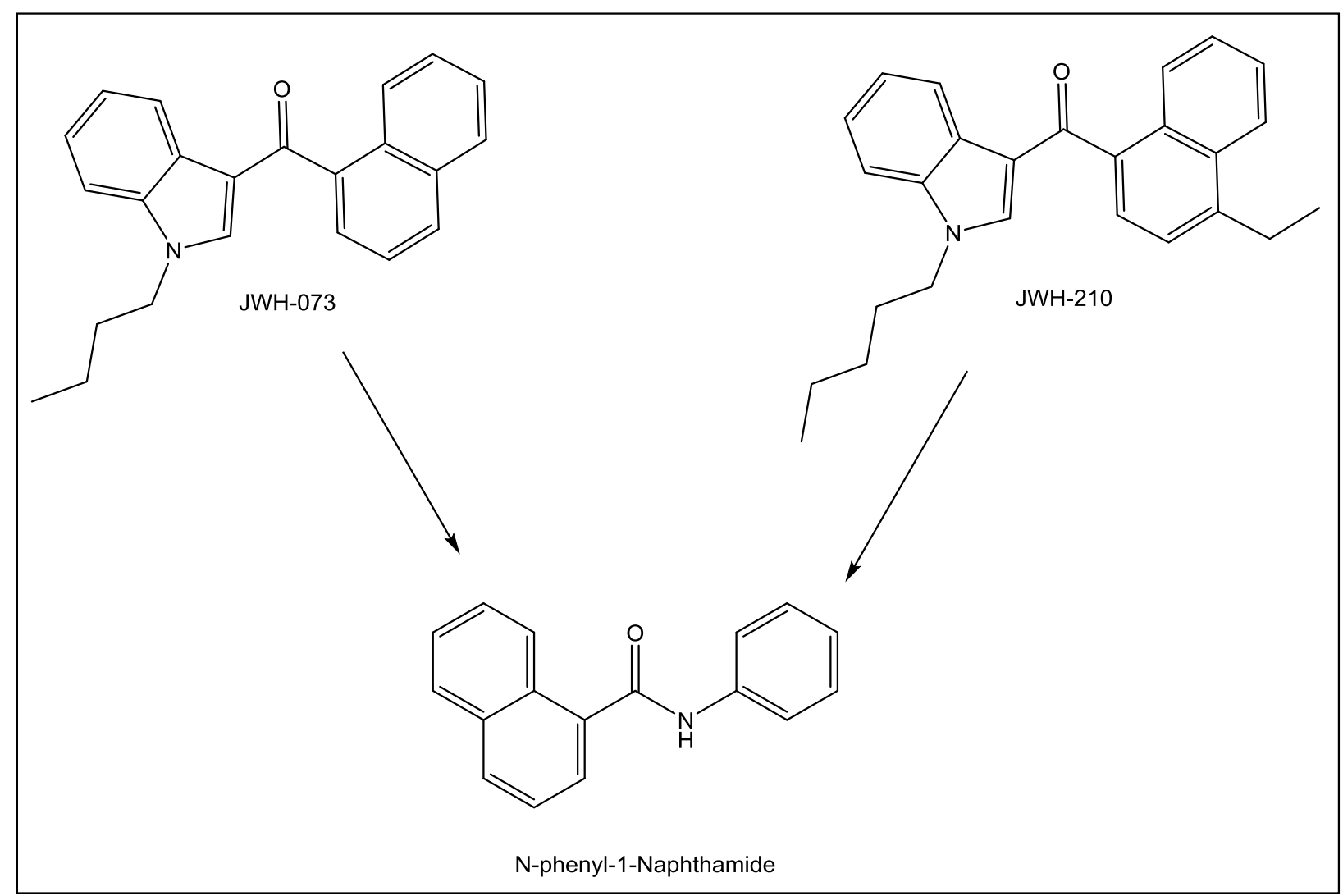

Figure C37 - Breakdown of parent synthetic cannabinoids that produced the proposed thermal degradant of N-phenyl-1-Naphthamide 


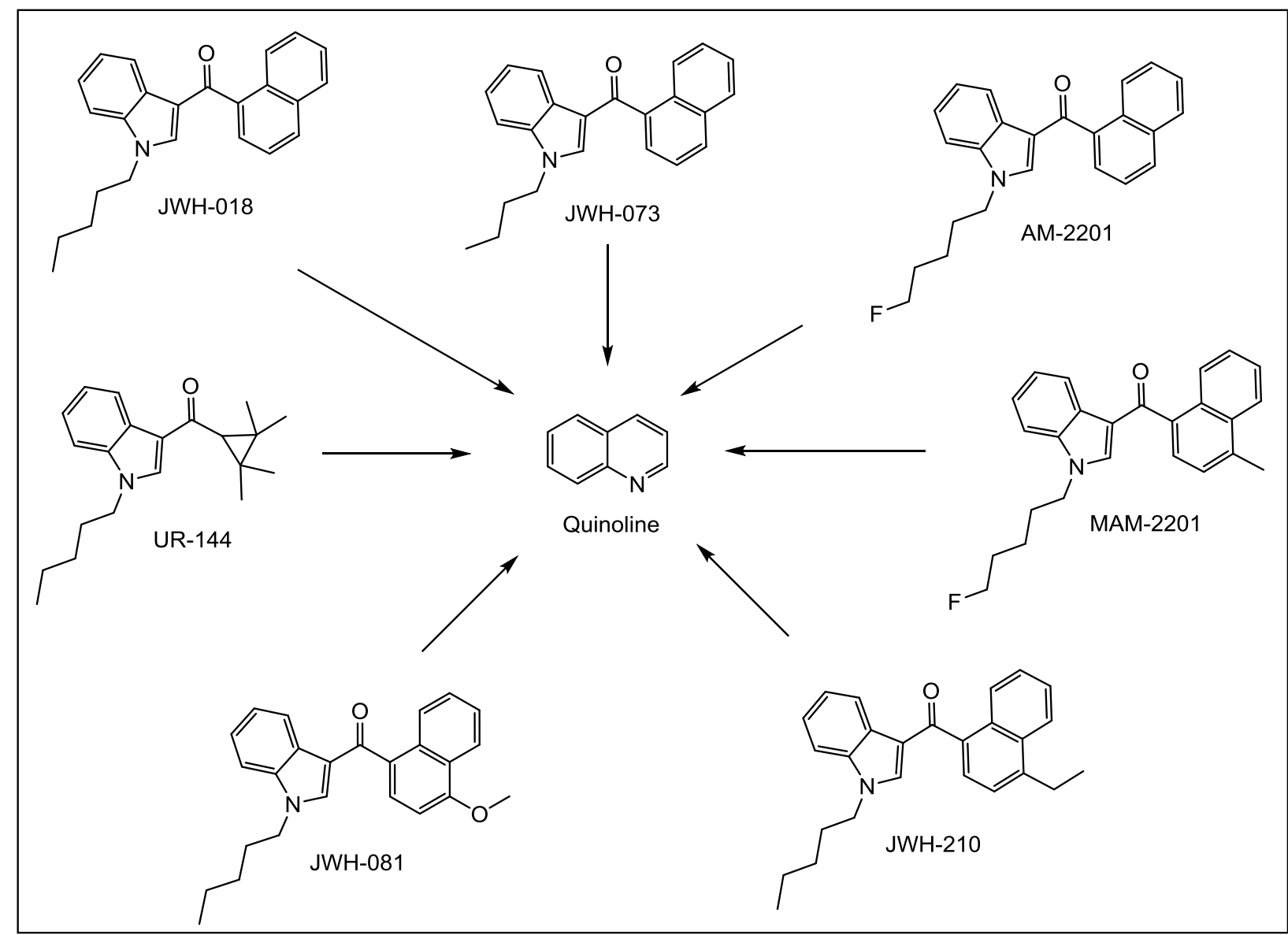

Figure C38 - Breakdown of parent synthetic cannabinoids that produced the proposed thermal degradant of Quinoline

\section{References}

1. Patterson, J. M., Mayer, C.F. and Smith Jr., W.T., Pyrolysis of Some Methyl- and Benzylindoles. J. Org. Chem. 1974, 40 (10), 1511-1514.

2. McNab, H., The mechanism of pyrolysis of 9, 9-dimethylfluorene. Arkivoc 2002, 6, 5966.

3. Karney, W. L. a. B., W.T., Ab Initio Study of the Ring Expansion of Phenylnitrene and Comparison with Ring Expansion of Phenylcarbene. J. Am. Chem. Soc. 1997, 119, 1378-1387.

4. Benson, S. W. a. O. N., H.E., Kinetic Data on Gas Phase Unimolecular Reactions. National Bureau of Standards: Washington, D.C., 1970; Vol. 21.

5. Moldoveanu, S. C., Pyrolysis of Organic Molecules with Applications to Health and Environmental Issues. Elsevier: Amsterdam, Netherlands, 2010; Vol. 28.

6. Vrček, I. V.; Vrček, V.; Siehl, H.-U., Quantum chemical study of degenerate hydride shifts in acyclic tertiary carbocations. The Journal of Physical Chemistry A 2002, 106 (8), 16041611. 


\section{Appendix D}

\section{Supplementary Material for Chapter 4: Simultaneous LC/MS/MS Monitoring of Synthetic Cannabinoids and Thermal Degradation Products in Postmortem Blood Samples}

Table D1 - Compound dependent parameters for the synthetic cannabinoids and observed pyrolytic products. * - predicted transition ions and parameters kept the same as cinnoline as the compounds differ by a single methyl group and a reference standard was unavailable

\begin{tabular}{|c|c|c|c|c|c|c|}
\hline \multirow{2}{*}{$\begin{array}{c}\text { Compound } \\
\text { Synthetic Cannabinoids }\end{array}$} & \multirow{2}{*}{$\begin{array}{c}\text { MRM } \\
\text { Transition (m/z) }\end{array}$} & \multicolumn{5}{|c|}{ Compound Dependent Parameters (V) } \\
\hline & & DP & EP & CEP & $\mathbf{C E}$ & CXP \\
\hline \multirow{2}{*}{$5 \mathrm{~F}-\mathrm{ADB}$} & $378.2 / 233.1$ & 77 & 6 & 37 & 30 & 4 \\
\hline & $378.1 / 213.2$ & 74 & 9 & 27 & 42 & 3 \\
\hline & & & & & & \\
\hline \multirow{2}{*}{$5 \mathrm{~F}-\mathrm{AMB}$} & $364.3 / 233.1$ & 85 & 5 & 33 & 30 & 4 \\
\hline & $364.3 / 213.2$ & 85 & 5 & 24 & 36 & 4 \\
\hline & & & & & & \\
\hline \multirow{2}{*}{$5 F-P B-22$} & $377.4 / 232.3$ & 60 & 5 & 45 & 31 & 6 \\
\hline & $377.4 / 144.2$ & 65 & 5 & 20 & 60 & 3 \\
\hline & & & & & & \\
\hline \multirow{2}{*}{ AB-CHMINACA } & $357.5 / 241.2$ & 80 & 10 & 37 & 40 & 4 \\
\hline & $357.5 / 144.9$ & 90 & 10 & 15 & 55 & 3 \\
\hline & & & & & & \\
\hline \multirow{2}{*}{ AB-FUBINACA } & $369.4 / 253.2$ & 65 & 6 & 35 & 30 & 4 \\
\hline & $369.4 / 109.2$ & 65 & 8 & 23 & 70 & 4 \\
\hline & & & & & & \\
\hline \multirow{2}{*}{ AB-PINACA } & $331.5 / 215.3$ & 75 & 5 & 36 & 32 & 8 \\
\hline & $331.5 / 145.3$ & 75 & 5 & 12 & 50 & 3 \\
\hline \multirow{2}{*}{ ADB-FUBINACA } & 38352529 & 00 & 5 & 33 & 32 & 9 \\
\hline & $383.5 / 108.8$ & 95 & 4 & 24 & 60 & 3 \\
\hline & & & & & & \\
\hline \multirow{2}{*}{ FUB-AKB-48 } & $404.5 / 135.1$ & 100 & 10 & 15 & 27 & 3 \\
\hline & $404.5 / 107.1$ & 95 & 10 & 20 & 65 & 3 \\
\hline \multirow{3}{*}{ MAB-CHMINACA } & & & & & & \\
\hline & $371.3 / 241.3$ & 95 & 5 & 14 & 37 & 4 \\
\hline & $371.3 / 145.1$ & 95 & 5 & 20 & 55 & 5 \\
\hline \multirow{2}{*}{ MMB-FUBINACA } & $384.4 / 253.1$ & 85 & 6 & 36 & 31 & 9 \\
\hline & $384.4 / 225.1$ & 85 & 6 & 26 & 43 & 4 \\
\hline
\end{tabular}




\begin{tabular}{|c|c|c|c|c|c|c|}
\hline Pyrolytic Products & & & & & & \\
\hline \multirow{2}{*}{ 1-Methylindazole } & $133.2 / 117.9$ & 58 & 9 & 11 & 32 & 3 \\
\hline & $133.2 / 91.2$ & 60 & 7 & 31 & 42 & 3 \\
\hline \multirow{2}{*}{ 4-Hydroxycinnoline } & $147.3 / 92.1$ & 67 & 11 & 12 & 29 & 2 \\
\hline & $147.3 / 75.2$ & 70 & 10 & 47 & 51 & 3 \\
\hline \multirow{2}{*}{$\begin{array}{l}\text { Indazole-3- } \\
\text { carboxaldehyde }\end{array}$} & $147.3 / 117.8$ & 50 & 8 & 13 & 38 & 3 \\
\hline & $147.3 / 91.8$ & 55 & 8 & 11 & 26 & 3 \\
\hline \multirow{2}{*}{ 1-Pentylindazole } & $189.2 / 133.5$ & 40 & 4 & 20 & 22 & 4 \\
\hline & $189.2 / 119.2$ & 40 & 4 & 15 & 35 & 3 \\
\hline \multirow{2}{*}{ Indazole } & $119.3 / 92.0$ & 52 & 11 & 11 & 30 & 3 \\
\hline & $119.3 / 65.8$ & 53 & 11 & 11 & 45 & 2 \\
\hline \multirow{2}{*}{ 4-Methylcinnoline* } & $144.2 / 102.2$ & 80 & 10 & 13 & 34 & 4 \\
\hline & $144.2 / 77.0$ & 80 & 10 & 12 & 37 & 3 \\
\hline \multirow{2}{*}{ Cinnoline } & $130.7 / 102.2$ & 80 & 10 & 13 & 34 & 4 \\
\hline & $130.7 / 77.0$ & 80 & 10 & 12 & 37 & 3 \\
\hline
\end{tabular}

Table D2 - Linearity, LOD, and LOQ values determined for each synthetic cannabinoid

\begin{tabular}{|c|c|c|c|}
\hline Compound & $\begin{array}{c}\text { Linearity Range } \\
(\mathbf{n g} / \mathbf{m l})\end{array}$ & $\mathbf{L O D}(\mathbf{n g} / \mathbf{m l})$ & $\mathbf{L O Q}(\mathbf{n g} / \mathbf{m l})$ \\
\hline 5F-ADB & $1.0-1,000.0$ & $0.1 \geq \mathrm{x} \leq 1.0$ & 10.0 \\
\hline 5F-AMB & $1.0-1,000.0$ & $1.0 \geq \mathrm{x} \leq 10.0$ & 10.0 \\
\hline 5F-PB-22 & $0.1-1,000.0$ & $0.01 \geq \mathrm{x} \leq 0.1$ & 1.0 \\
\hline AB-CHMINACA & $1.0-1,000.0$ & $0.1 \geq \mathrm{x} \leq 1.0$ & 10.0 \\
\hline AB-FUBINACA & $1.0-1,000.0$ & $0.1 \geq \mathrm{x} \leq 1.0$ & 10.0 \\
\hline AB-PINACA & $1.0-1,000.0$ & $0.1 \geq \mathrm{x} \leq 1.0$ & 10.0 \\
\hline ADB-FUBINACA & $1.0-1,000.0$ & $0.1 \geq \mathrm{x} \leq 1.0$ & 10.0 \\
\hline FUB-AKB-48 & $1.0-1,000.0$ & $1.0 \geq \mathrm{x} \leq 10.0$ & 10.0 \\
\hline MAB-CHMINACA & $1.0-1,000.0$ & $0.1 \geq \mathrm{x} \leq 1.0$ & 10.0 \\
\hline MMB-FUBINACA & $1.0-1,000.0$ & $1.0 \geq \mathrm{x} \leq 10.0$ & 10.0 \\
\hline
\end{tabular}


Table D3 - Precision and accuracy data for both within-day and between-day evaluations

\begin{tabular}{|c|c|c|c|c|c|c|c|c|c|c|c|c|}
\hline \multirow{3}{*}{ Parent Compound } & \multicolumn{6}{|c|}{ Precision (\% CV) } & \multicolumn{6}{|c|}{ Accuracy (avg. $\%$ difference, $\pm \% \mathrm{CV}$ of $\overline{\mathbf{x}}$ ) } \\
\hline & \multicolumn{3}{|c|}{ Within-day; $n=5$} & \multicolumn{3}{|c|}{ Between-day; $n=10$} & \multicolumn{3}{|c|}{ Within-day; n=5 } & \multicolumn{3}{|c|}{ Between-day; $\mathbf{n = 1 0}$} \\
\hline & Low & Mid & High & Low & Mid & High & Low & Mid & High & Low & Mid & High \\
\hline $5 \mathrm{~F}-\mathrm{ADB}$ & 1.7 & 7.0 & 20.3 & 1.6 & 7.6 & 18.2 & 11.9 & 11.4 & 10.1 & 17.4 & 11.4 & 0.3 \\
\hline $5 \mathrm{~F}-\mathrm{AMB}$ & 0.9 & 6.4 & 6.7 & 1.2 & 6.8 & 18.8 & 15.9 & 10.9 & 0.0 & 4.4 & 7.4 & 2.5 \\
\hline 5F-PB-22 & 8.8 & 9.7 & 17.3 & 8.9 & 8.6 & 12.5 & 3.8 & 1.6 & 6.2 & 2.7 & 0.6 & 0.0 \\
\hline AB-CHMINACA & 5.6 & 8.4 & 10.3 & 7.9 & 9.3 & 9.2 & 1.4 & 1.9 & 4.7 & 12.0 & 0.5 & 0.1 \\
\hline AB-FUBINACA & 6.5 & 8.5 & 19.4 & 6.7 & 9.0 & 15.3 & 0.5 & 2.4 & 4.5 & 9.2 & 0.9 & 0.4 \\
\hline AB-PINACA & 13.4 & 19.5 & 20.7 & 14.2 & 11.9 & 20.5 & 15.7 & 13.5 & 14.8 & 0.5 & 0.0 & 0.0 \\
\hline ADB-FUBINACA & 0.9 & 3.1 & 10.3 & 0.8 & 3.6 & 18.8 & 14.4 & 7.4 & 19.0 & 4.2 & 4.8 & 0.0 \\
\hline FUB-AKB-48 & 8.4 & 16.5 & 17.1 & 8.3 & 16.3 & 20.9 & 17.0 & 4.6 & 4.1 & 4.2 & 3.4 & 1.5 \\
\hline MAB-CHMINACA & 7.3 & 10.9 & 13.3 & 14.6 & 11.0 & 18.3 & 1.7 & 18.2 & 6.2 & 17.8 & 17.5 & 1.5 \\
\hline MMB-FUBINACA & 0.5 & 6.1 & 9.7 & 0.9 & 9.2 & 18.6 & 3.1 & 13.6 & 5.7 & 5.6 & 21.9 & 0.4 \\
\hline
\end{tabular}

Table D4 - Average extraction recoveries and matrix effects; analyzed at $\mathrm{n}=3$

\begin{tabular}{|c|c|c|c|c|}
\hline \multirow{2}{*}{ Parent Compound } & \multicolumn{2}{|c|}{ Matrix Effect } & \multicolumn{2}{c|}{ Recovery } \\
\cline { 2 - 5 } & $\mathbf{5 0} \mathbf{~ n g} / \mathbf{m l}$ & $\mathbf{5 0 0} \mathbf{~ n g} / \mathbf{m l}$ & $\mathbf{5 0 ~ n g / m l}$ & $\mathbf{5 0 0} \mathbf{~ g} / \mathbf{m l}$ \\
\hline 5F-ADB & 76.9 & 67.9 & 97.4 & 86.4 \\
\hline 5F-AMB & 112.3 & 59.8 & 70.2 & 77.6 \\
\hline 5F-PB-22 & 41.9 & 98.3 & 136.2 & 130.0 \\
\hline AB-CHMINACA & 81.8 & 155.7 & 78.8 & 165.2 \\
\hline AB-FUBINACA & 97.1 & 79.6 & 86.5 & 64.7 \\
\hline AB-PINACA & 85.1 & 80.5 & 141.9 & 73.4 \\
\hline ADB-FUBINACA & 136.1 & 92.3 & 129.6 & 131.8 \\
\hline FUB-AKB-48 & 85.4 & 80.8 & 76.7 & 65.5 \\
\hline MAB-CHMINACA & 105.2 & 113.2 & 105.7 & 119.0 \\
\hline MMB-FUBINACA & 139.6 & 84.2 & 89.9 & 67.3 \\
\hline
\end{tabular}




\section{Appendix E}

\section{Supporting data that was not included in the manuscript for chapter 4.}

Predicted pyrolytic products of the synthetic cannabinoids present in the obtained case blood samples:

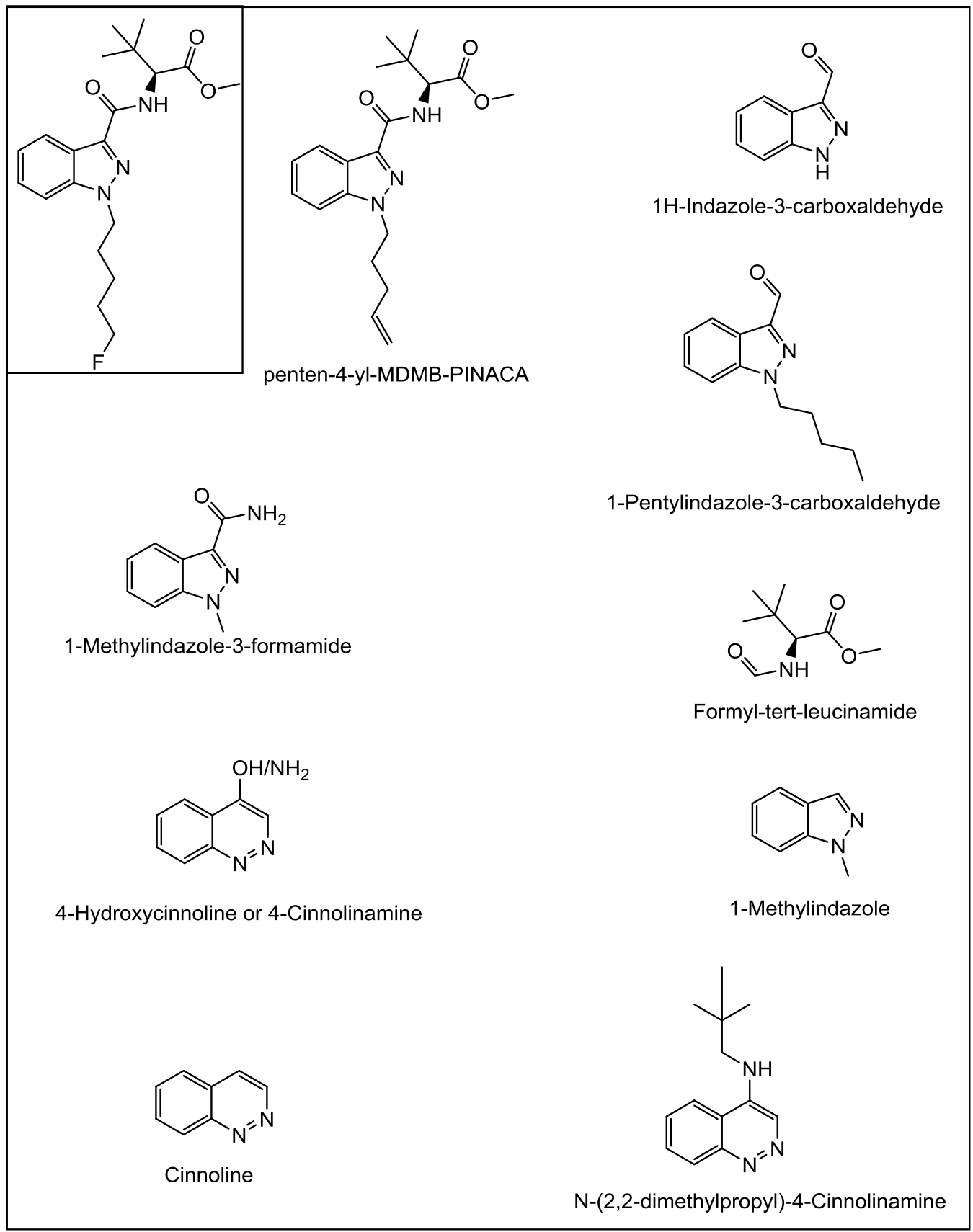

Figure E1 - Possible thermal degradation products for the parent synthetic cannabinoid, 5F-ADB 


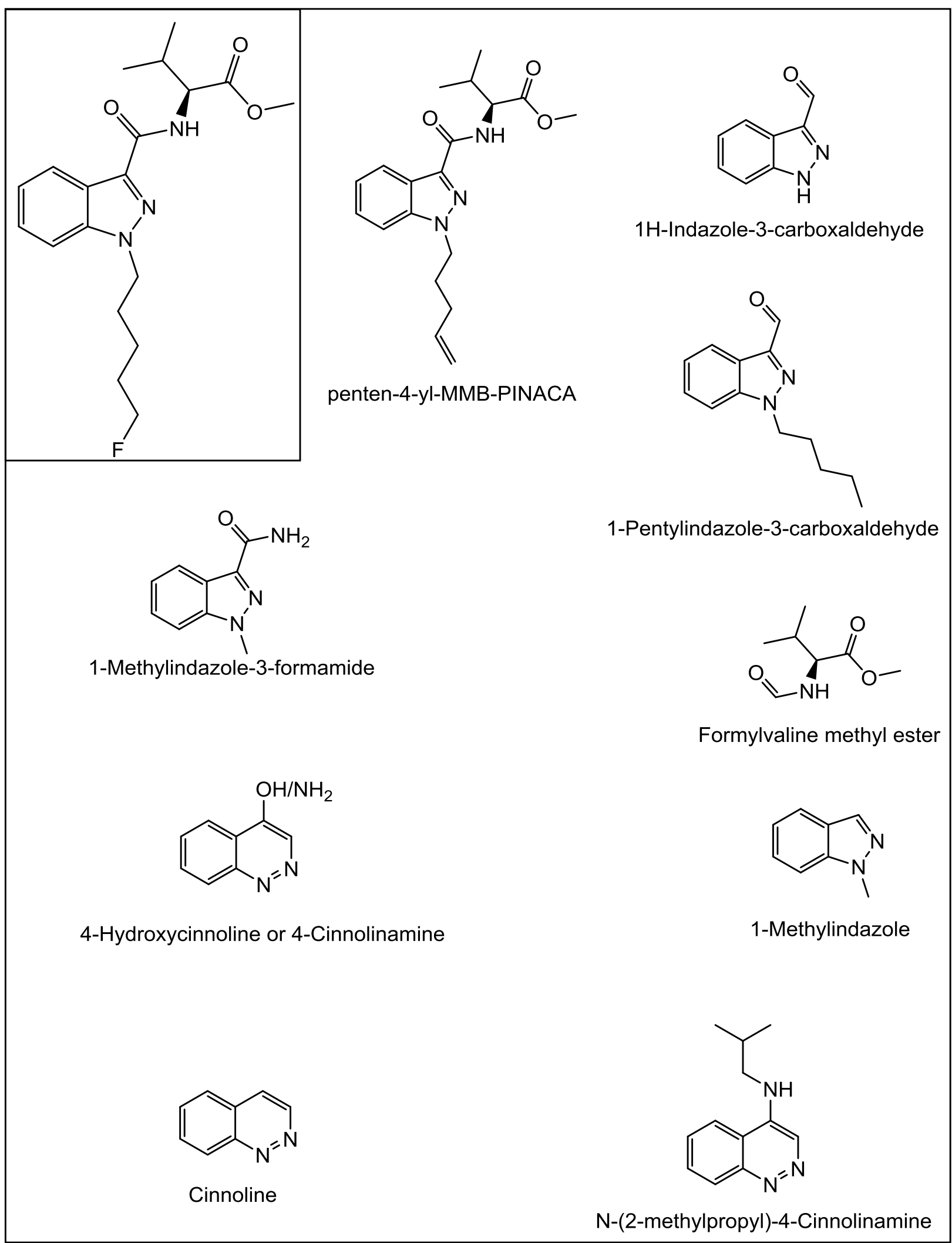

Figure E2 - Possible thermal degradation products for the parent synthetic cannabinoid, 5F-AMB 


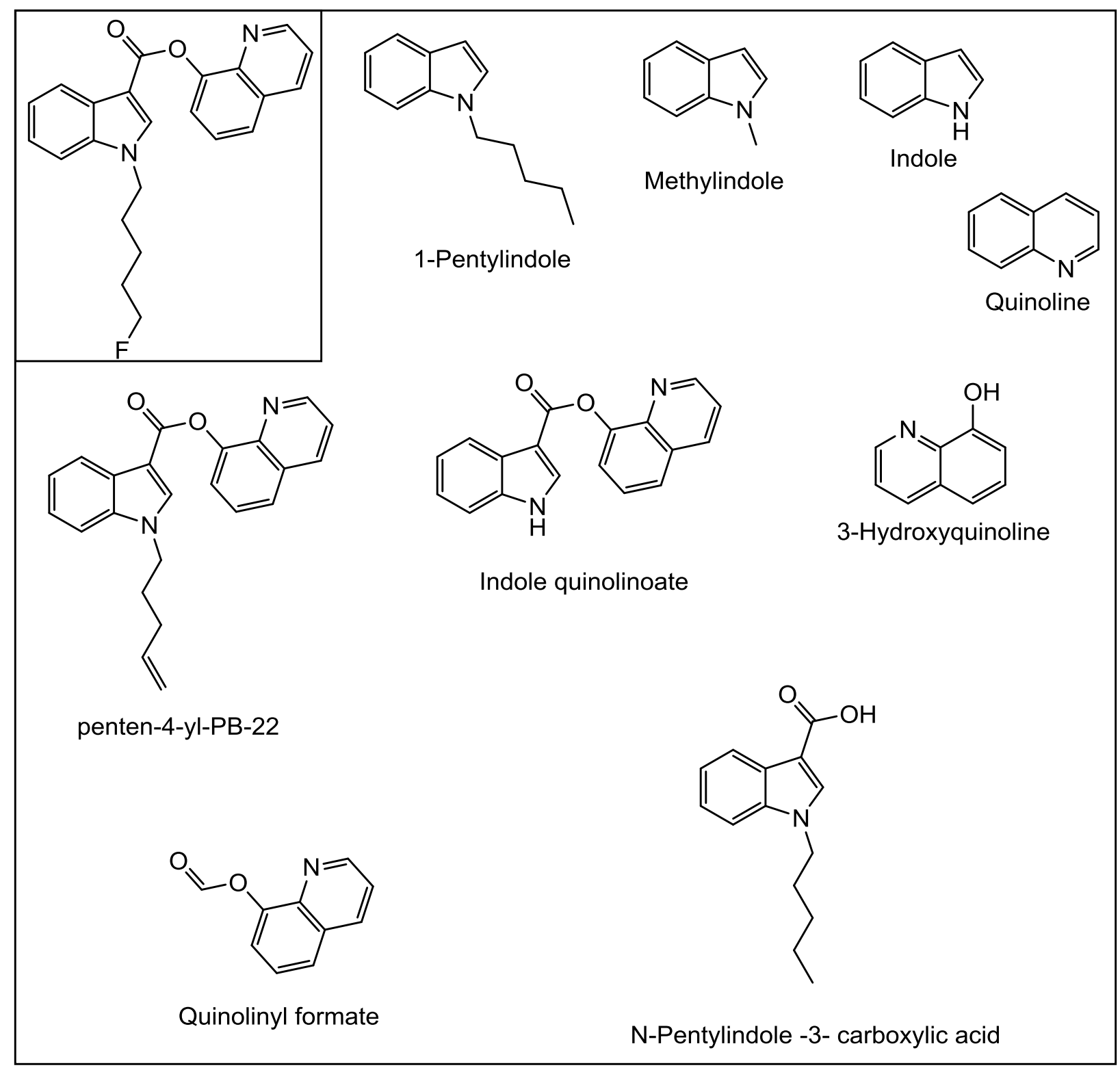

Figure E3 - Possible thermal degradation products for the parent synthetic cannabinoid, 5F-PB-22 


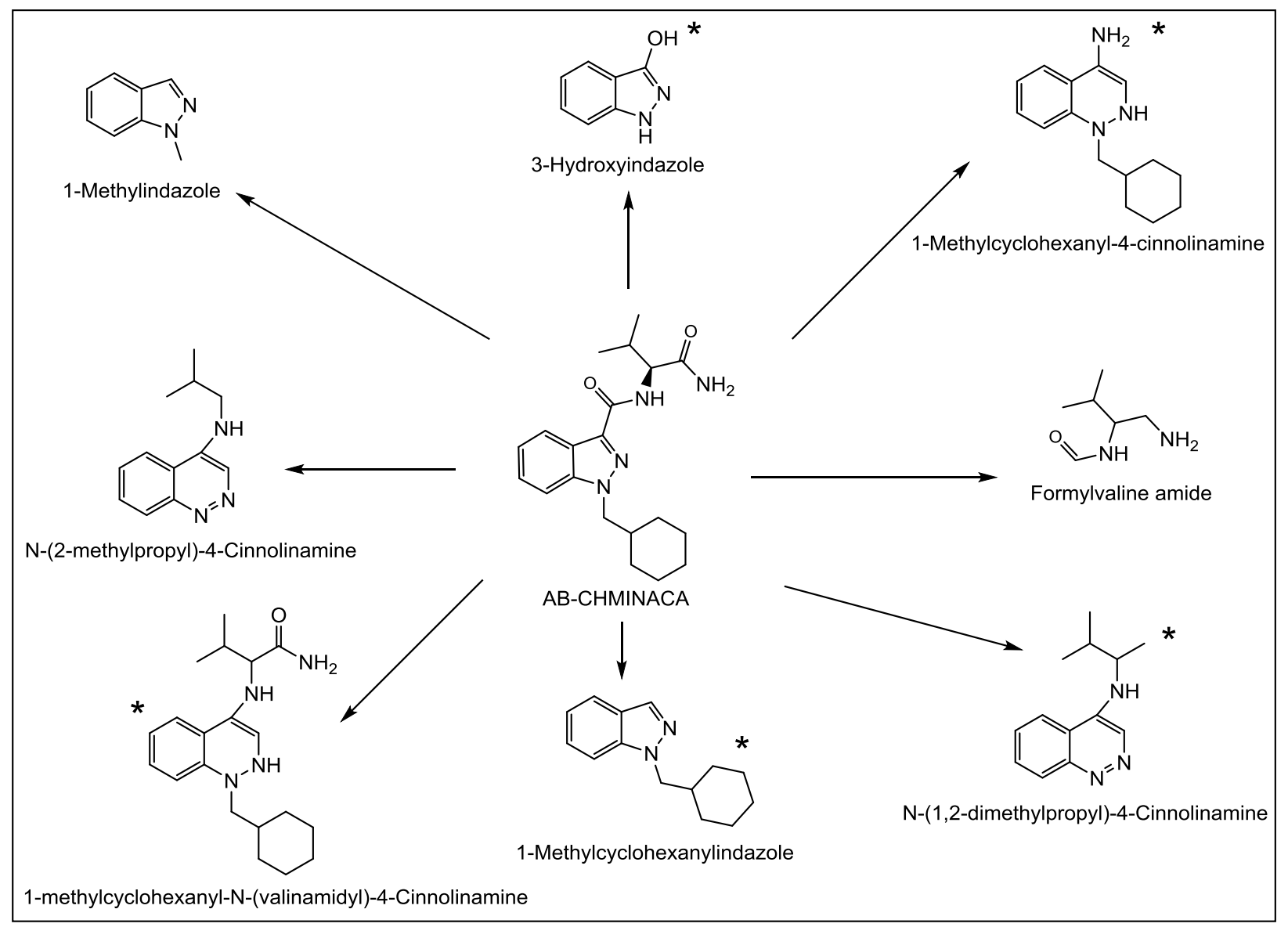

Figure E4 - Possible thermal degradation products for the parent synthetic cannabinoid, ABCHMINACA 


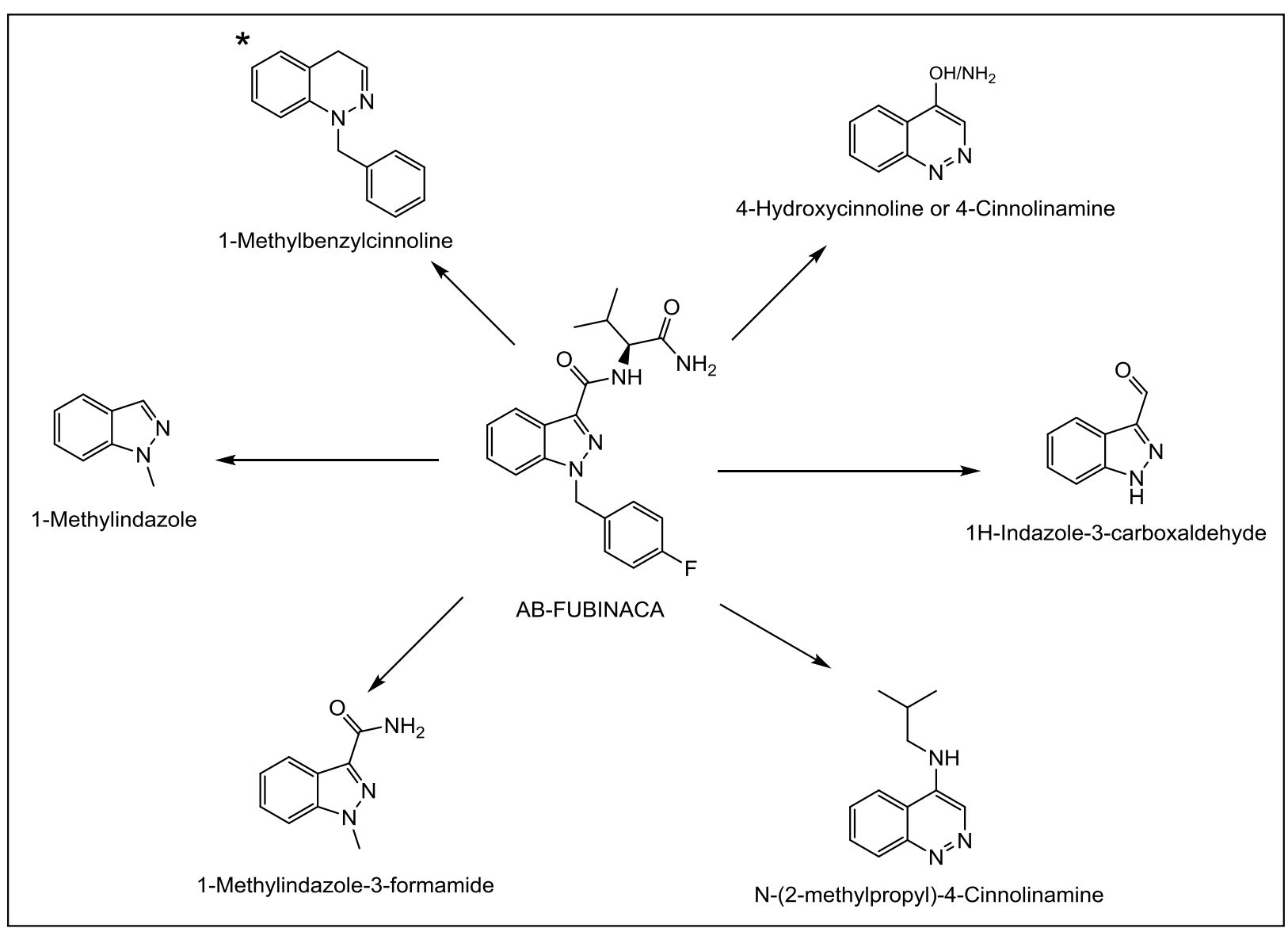

Figure E5 - Possible thermal degradation products for the parent synthetic cannabinoid, ABFUBINACA 


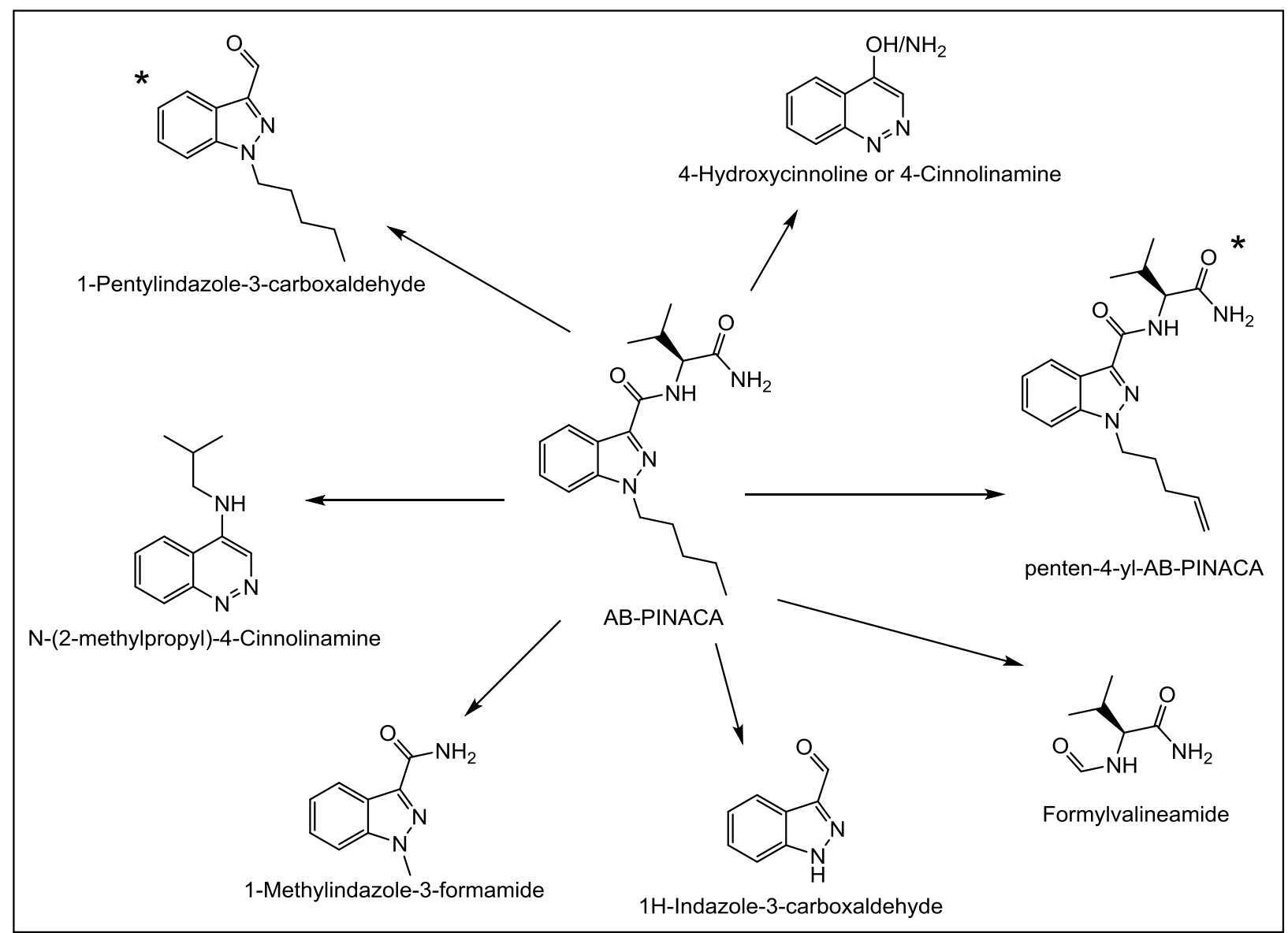

Figure E6 - Possible thermal degradation products for the parent synthetic cannabinoid, AB-PINACA 


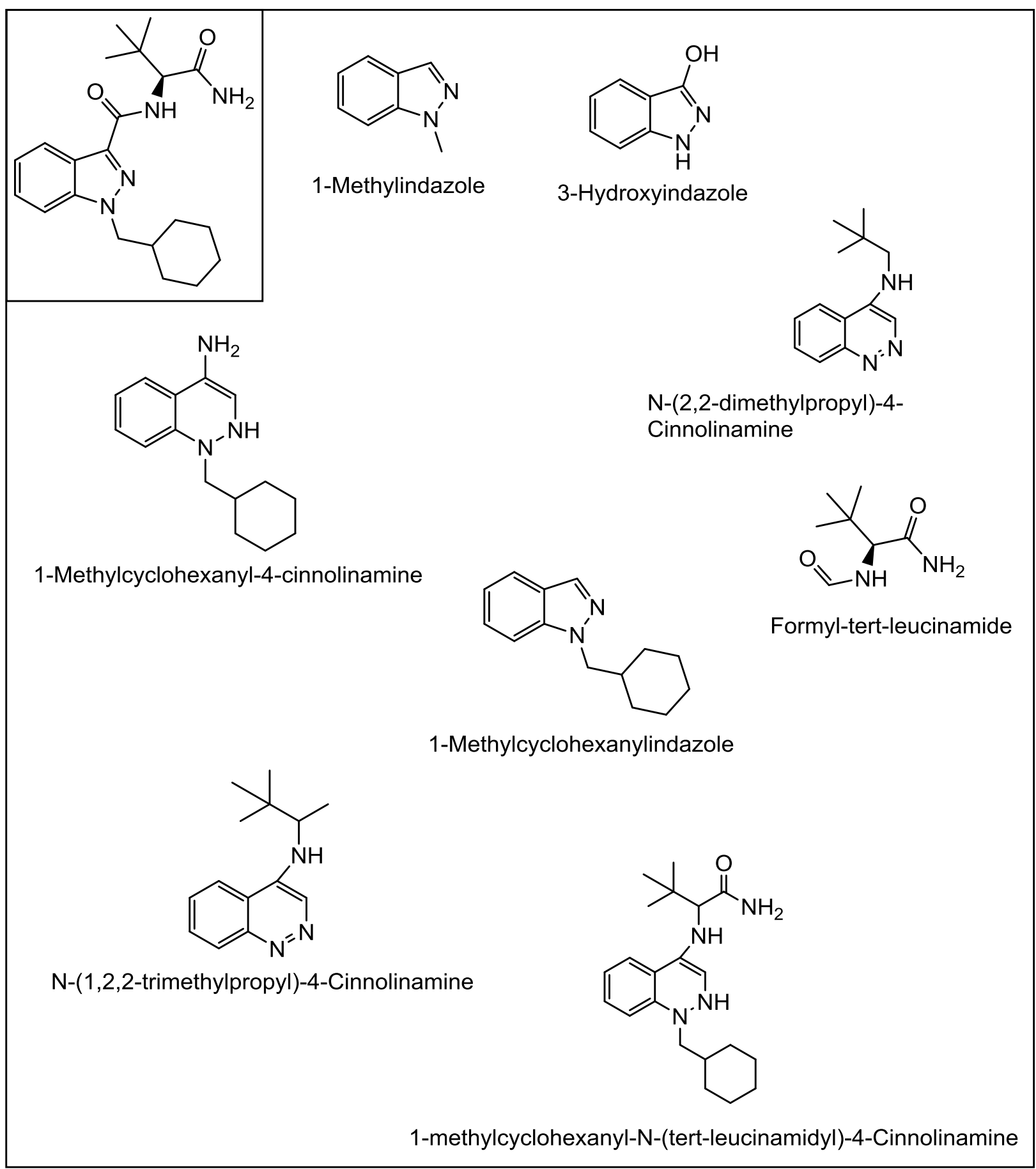

Figure E7 - Possible thermal degradation products for the parent synthetic cannabinoid, ADB-CHMINACA (MAB-CHMINACA) 


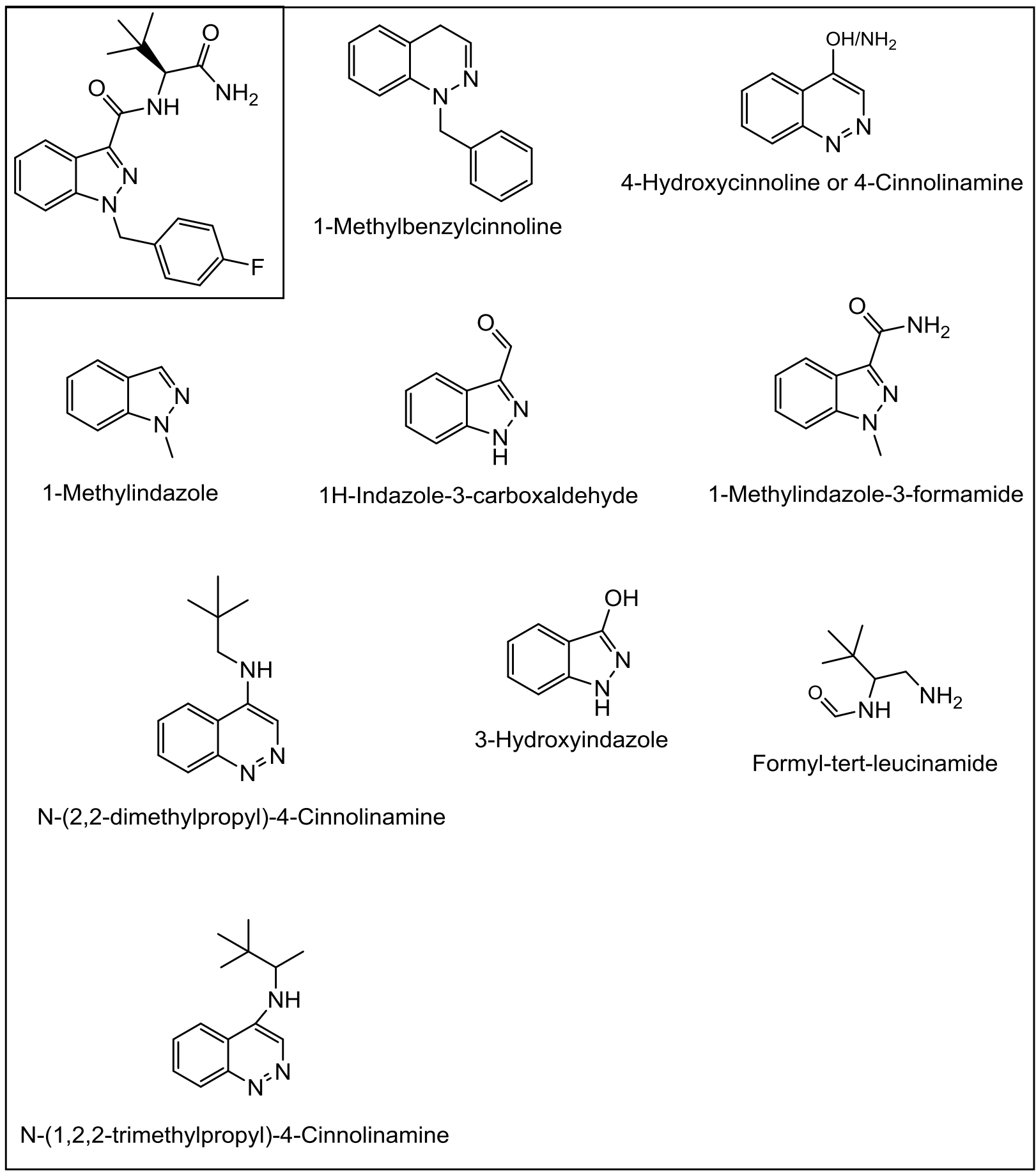

Figure E8 - Possible thermal degradation products for the parent synthetic cannabinoid, ADB-FUBINACA 


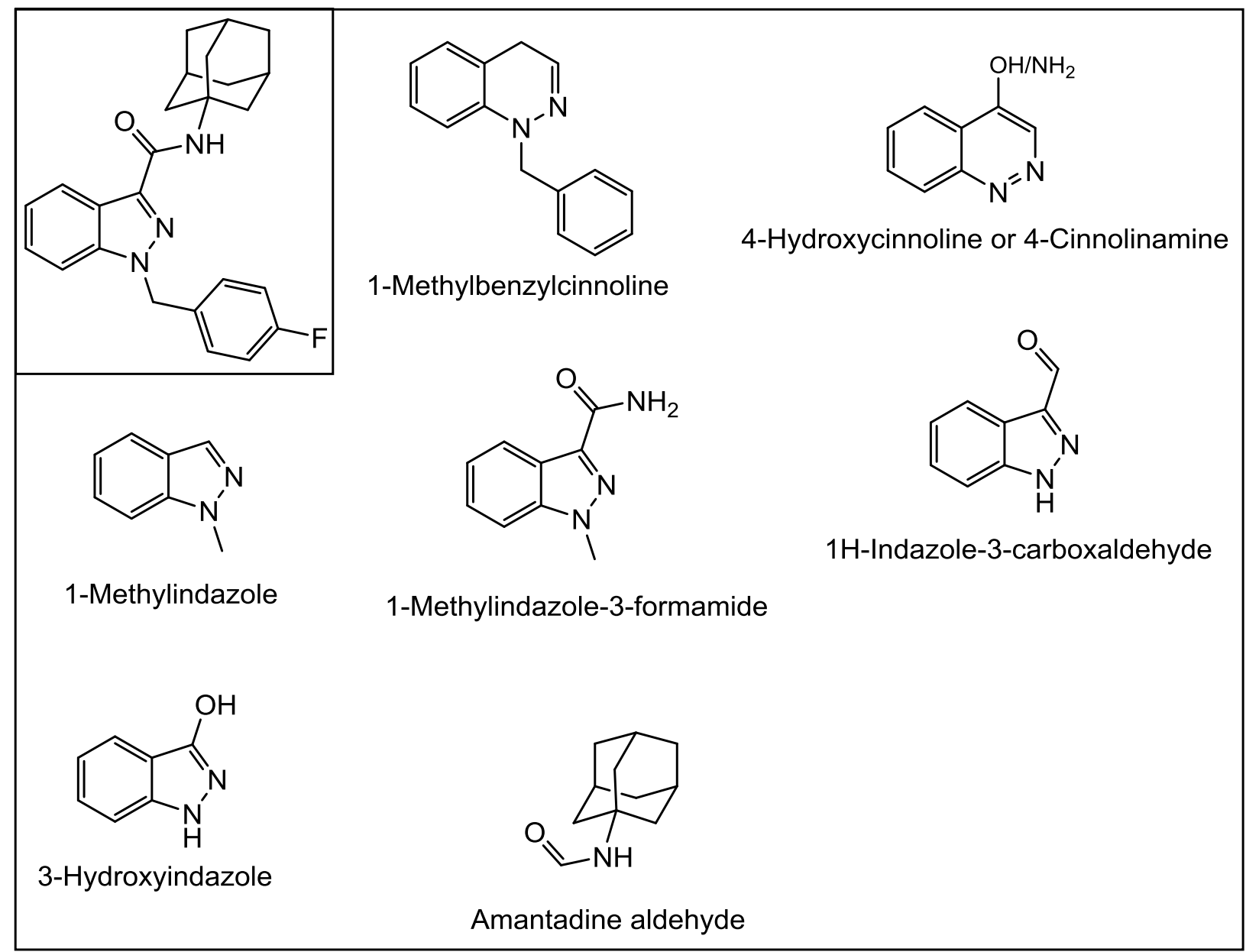

Figure E9 - Possible thermal degradation products for the parent synthetic cannabinoid, FUB-AKB-48 


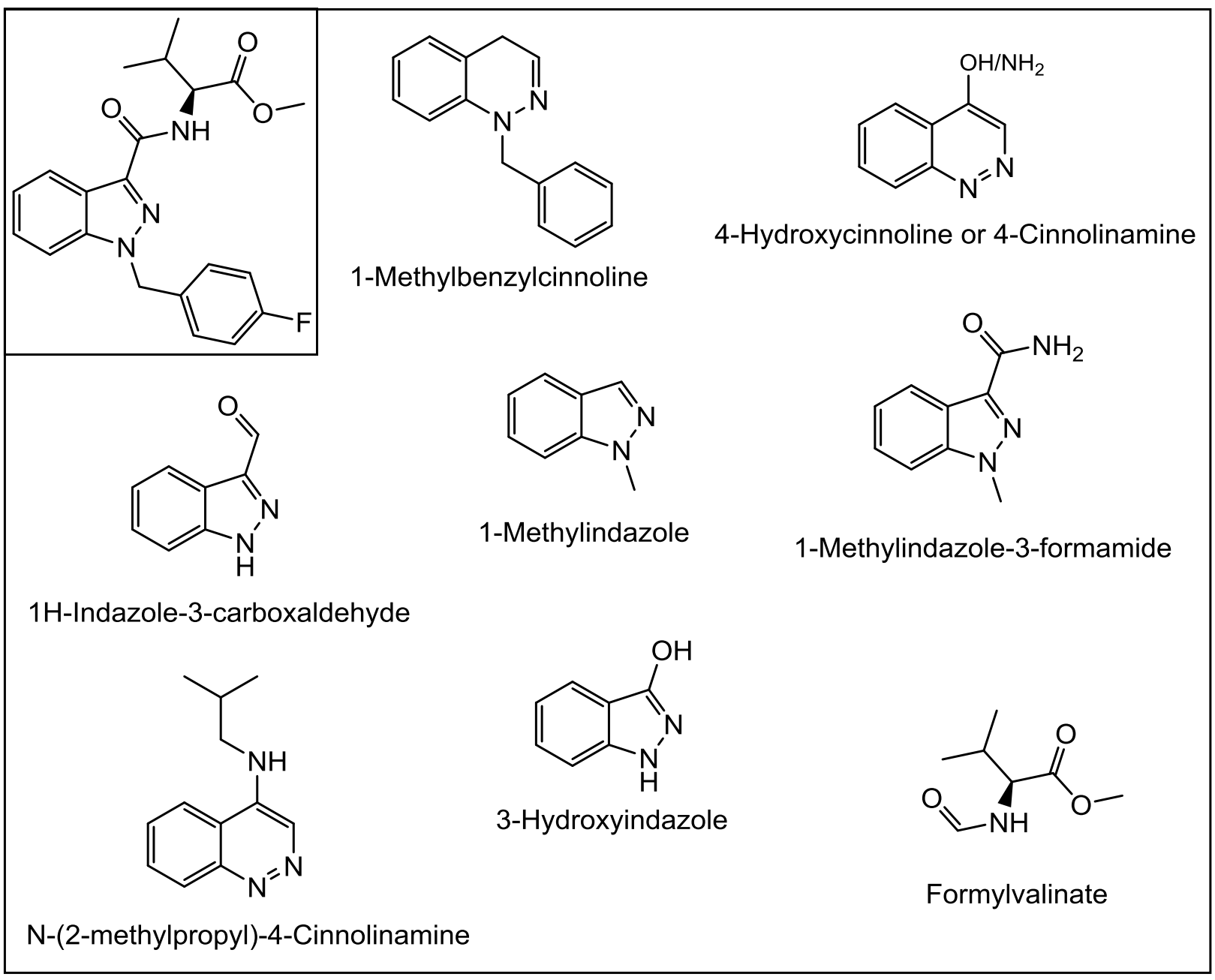

Figure E10 - Possible thermal degradation products for the parent synthetic cannabinoid, MMB-FUBINACA 
Method validation linearity graphs and example chromatographs:

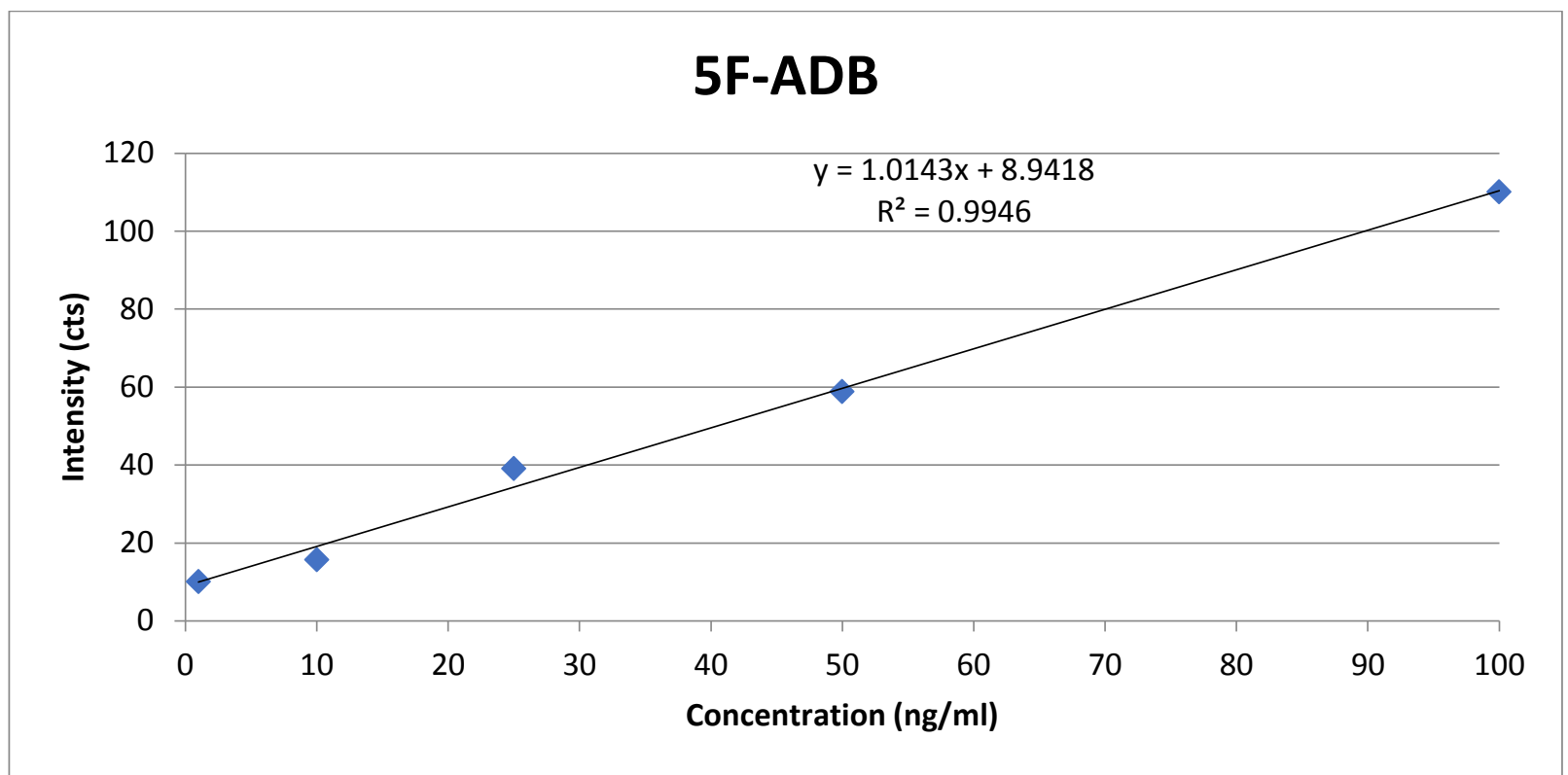

Figure E11 - Linearity plot of concentration $(1-100 \mathrm{ng} / \mathrm{ml})$ vs intensity for 5F-ADB

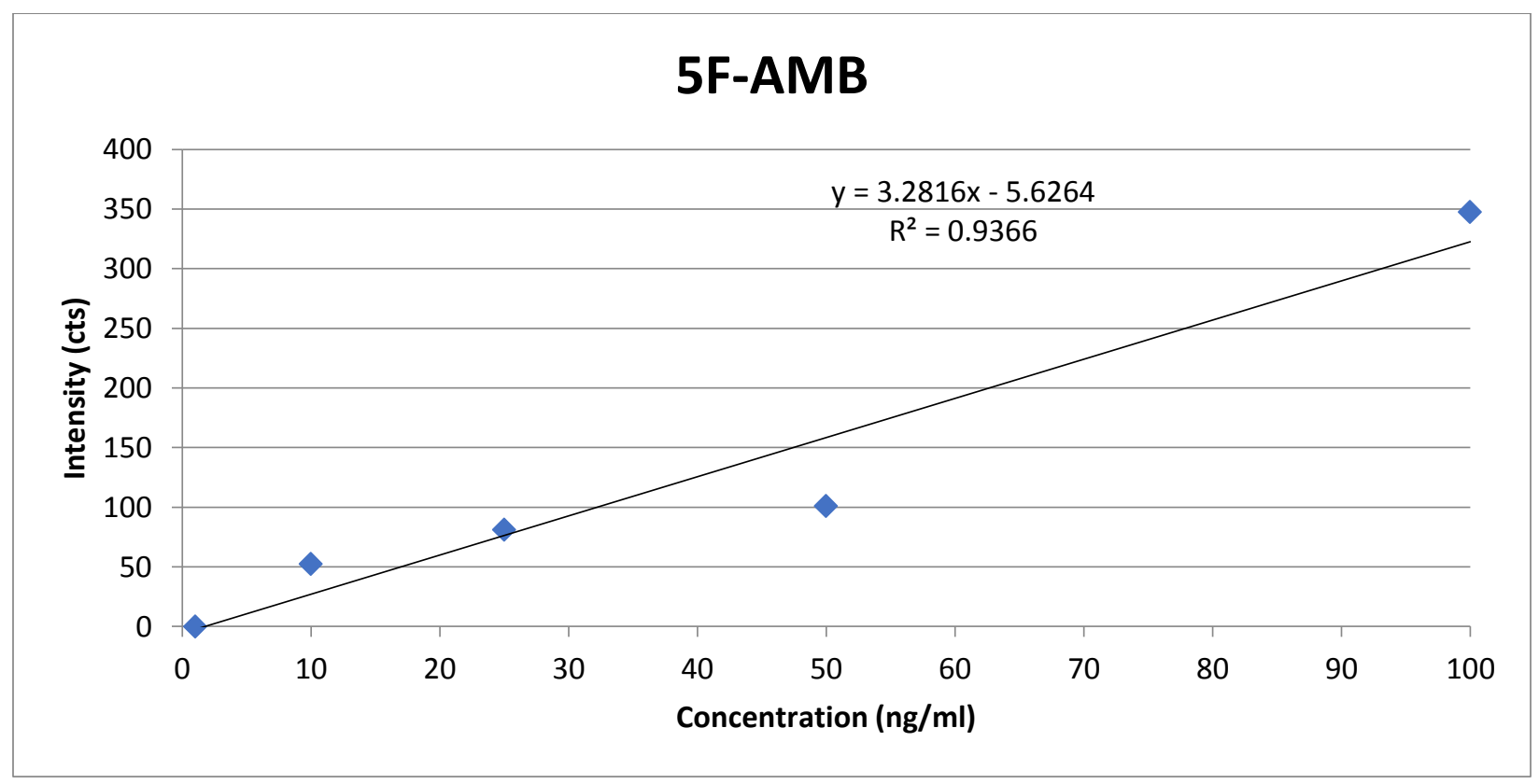

Figure E12 - Linearity plot of concentration $(1-100 \mathrm{ng} / \mathrm{ml})$ vs intensity for 5F-AMB 


\section{F-PB-22}

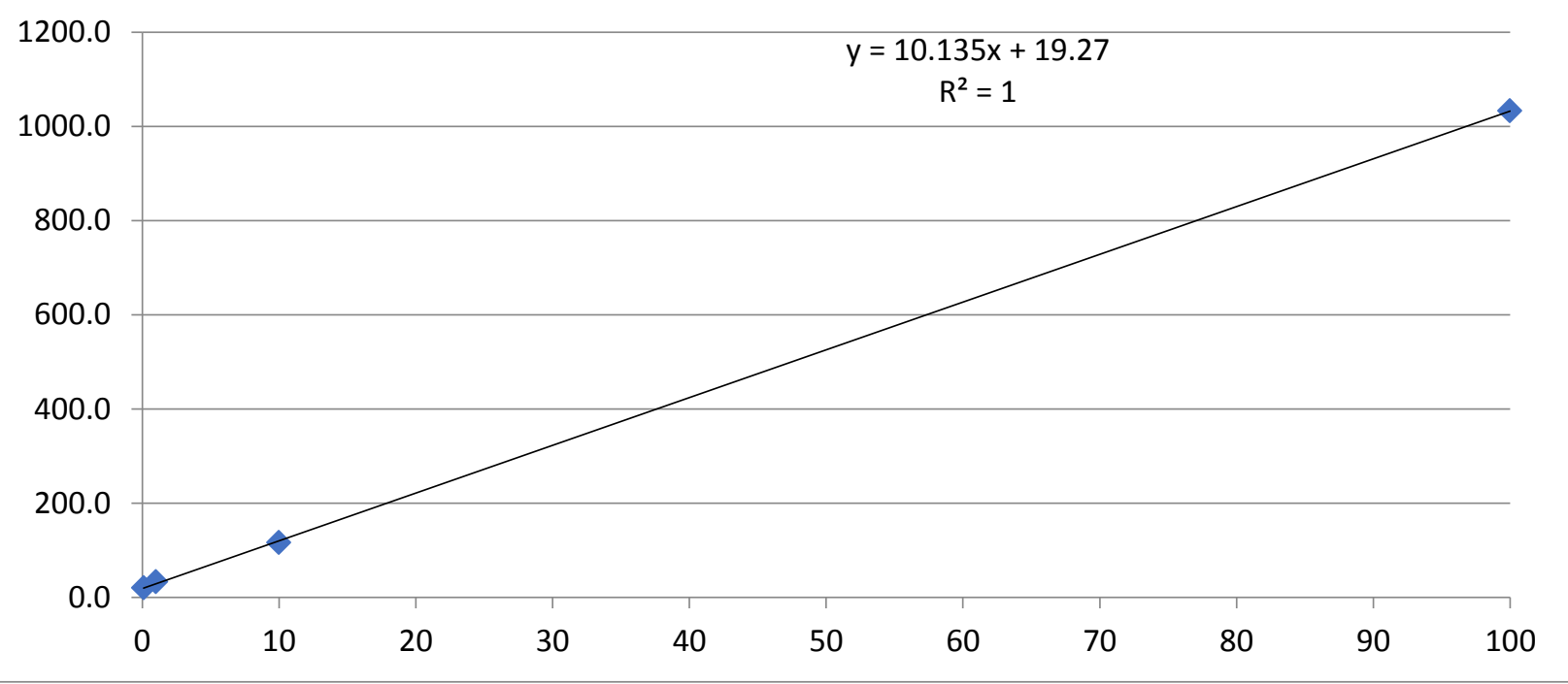

Figure E13 - Linearity plot of concentration $(0.1-100 \mathrm{ng} / \mathrm{ml})$ vs intensity for 5F-PB-22

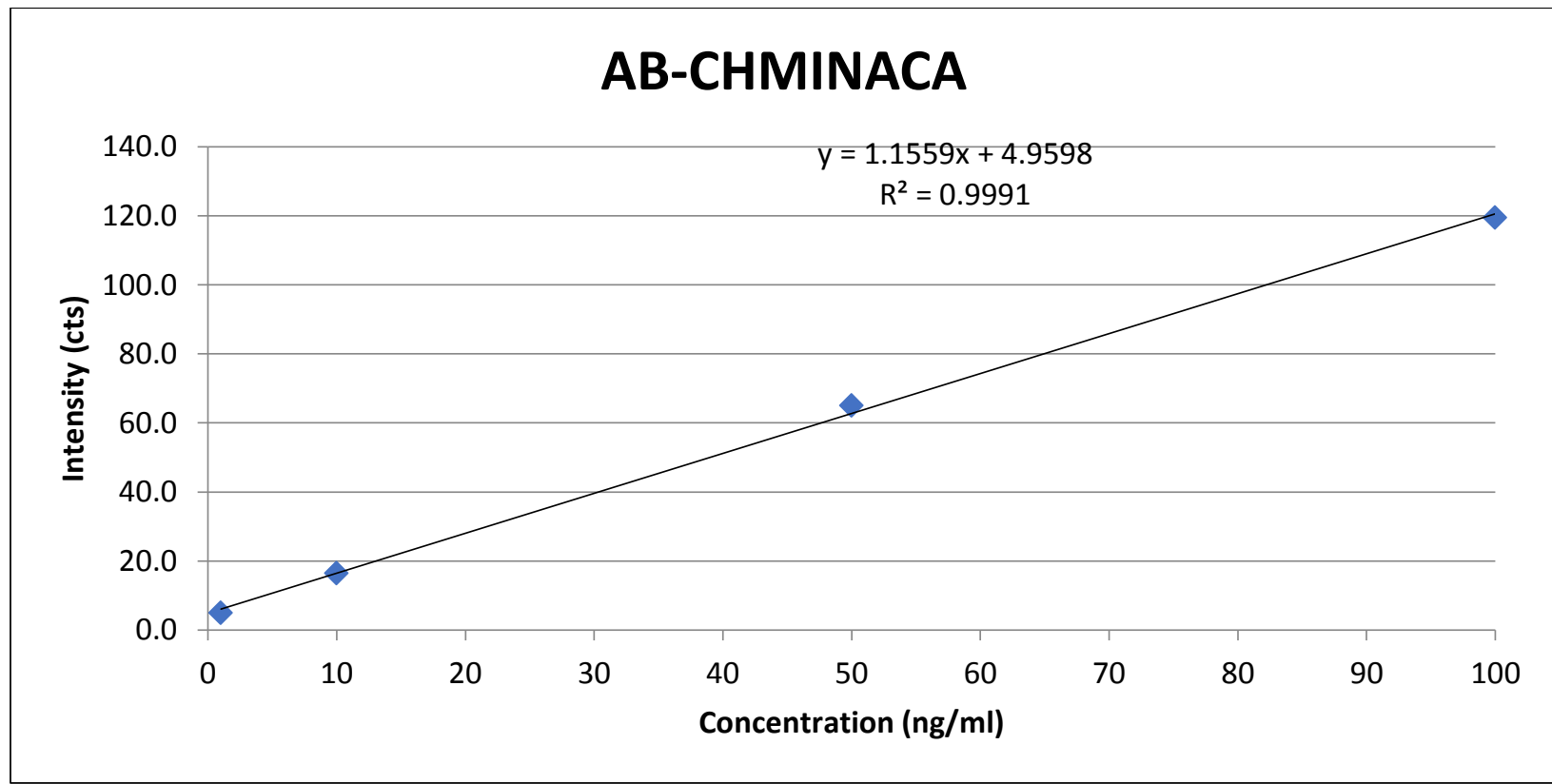

Figure E14 - Linearity plot of concentration $(1-100 \mathrm{ng} / \mathrm{ml})$ vs intensity for AB-CHMINACA 


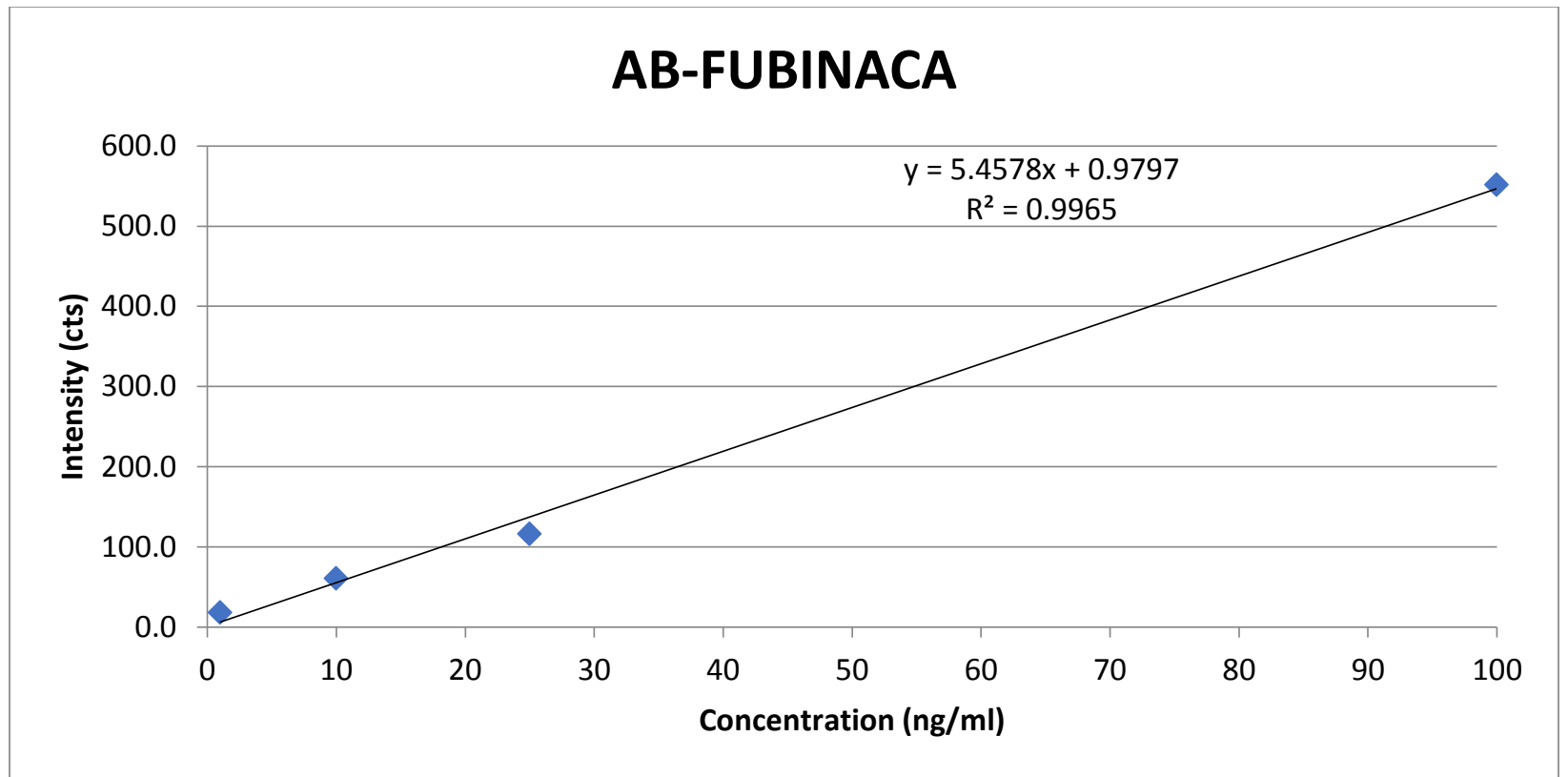

Figure E15 - Linearity plot of concentration $(1-100 \mathrm{ng} / \mathrm{ml})$ vs intensity for AB-FUBINACA

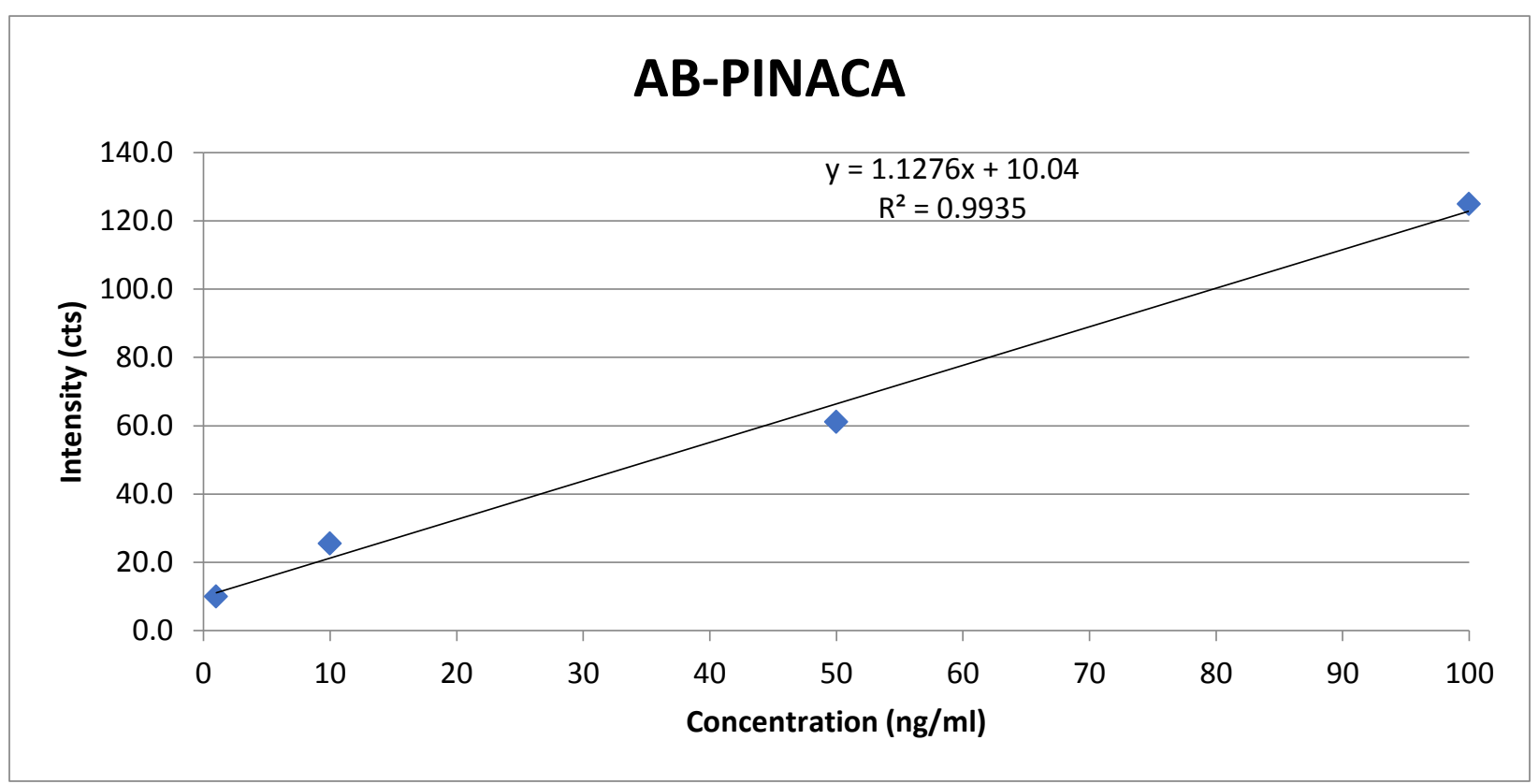

Figure E16 - Linearity plot of concentration $(1-100 \mathrm{ng} / \mathrm{ml})$ vs intensity for AB-PINACA 


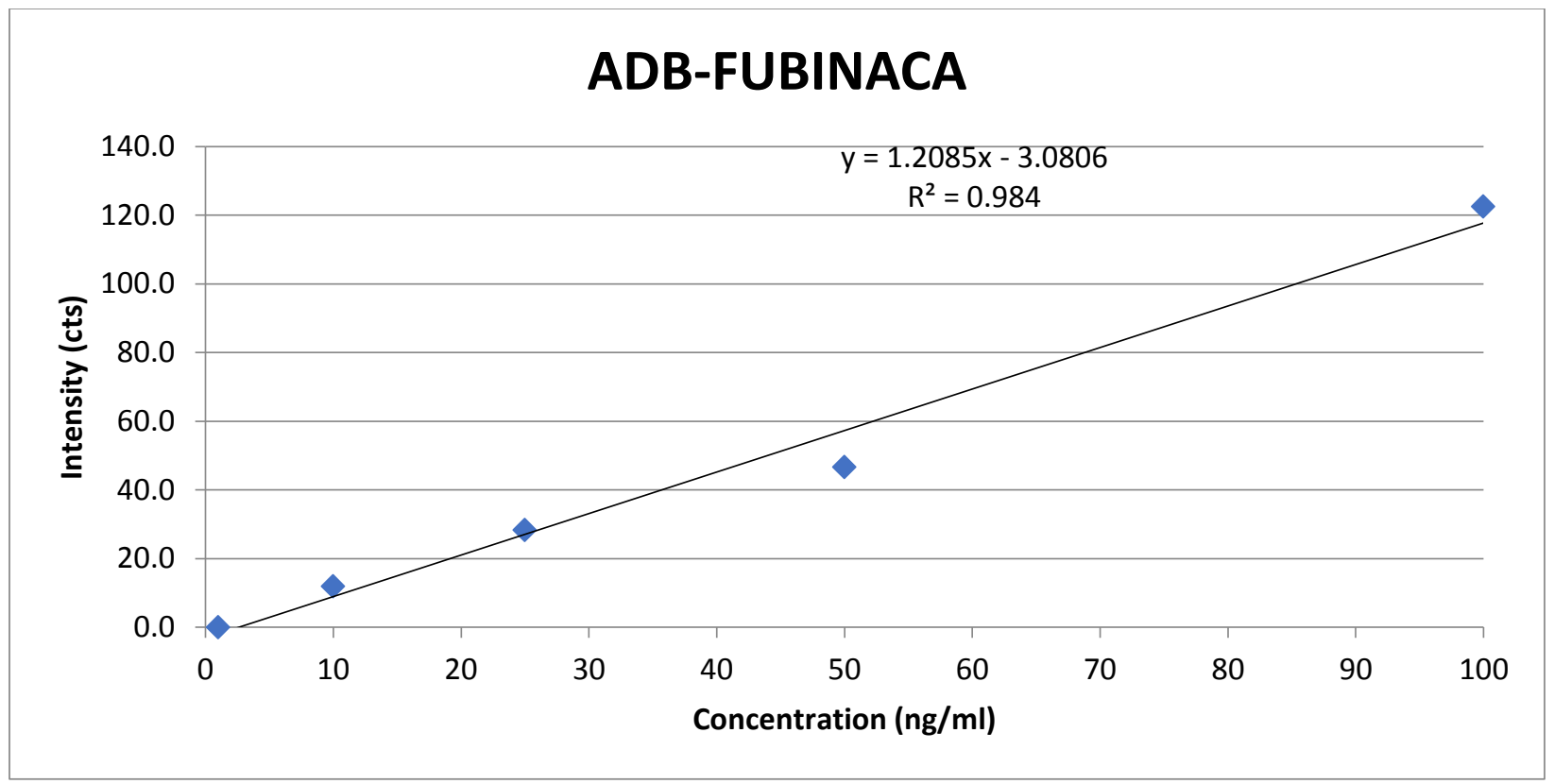

Figure E17 - Linearity plot of concentration $(1-100 \mathrm{ng} / \mathrm{ml})$ vs intensity for ADB-FUBINACA

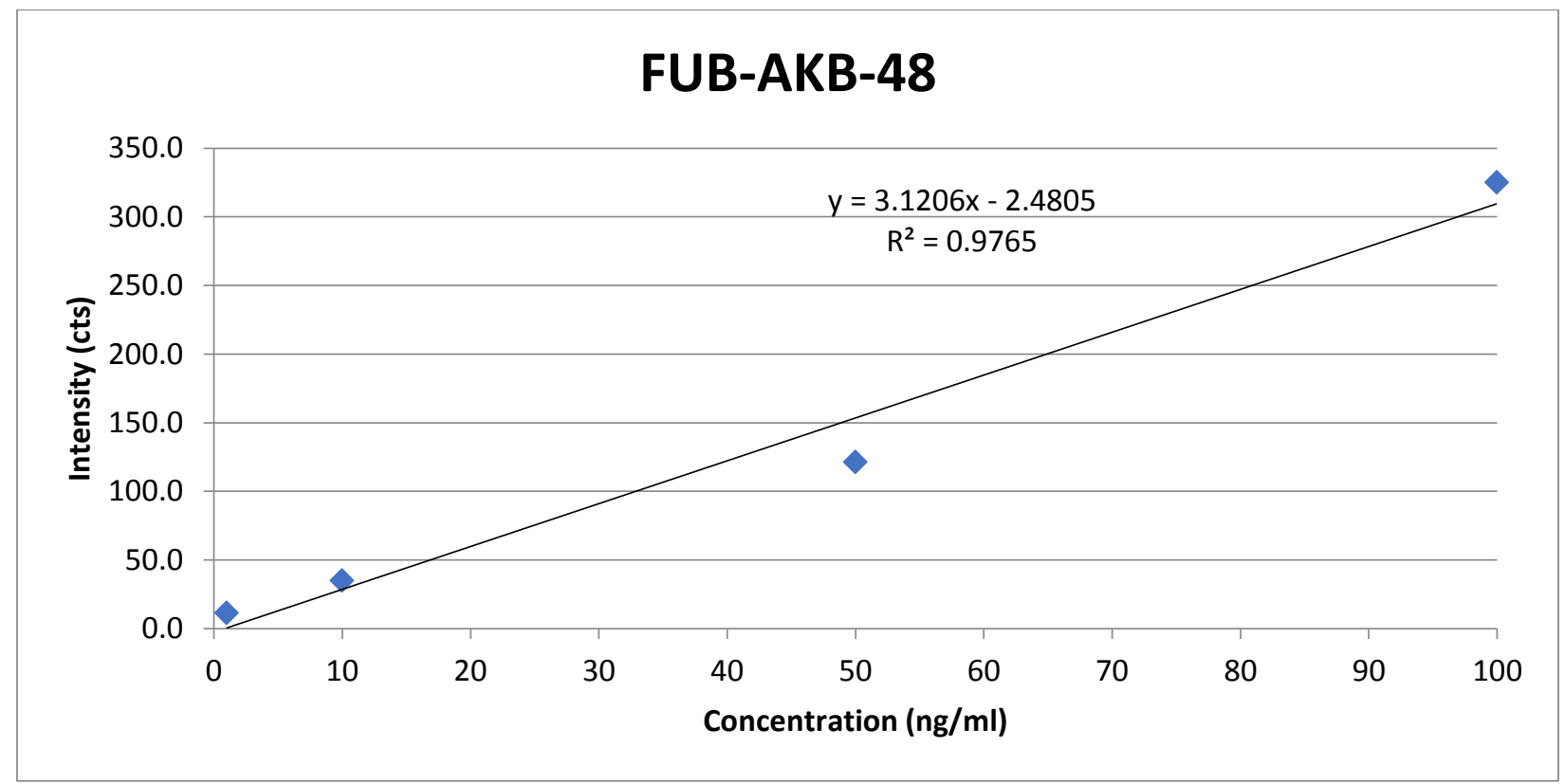

Figure E18 - Linearity plot of concentration $(1-100 \mathrm{ng} / \mathrm{ml})$ vs intensity for FUB-AKB-48 


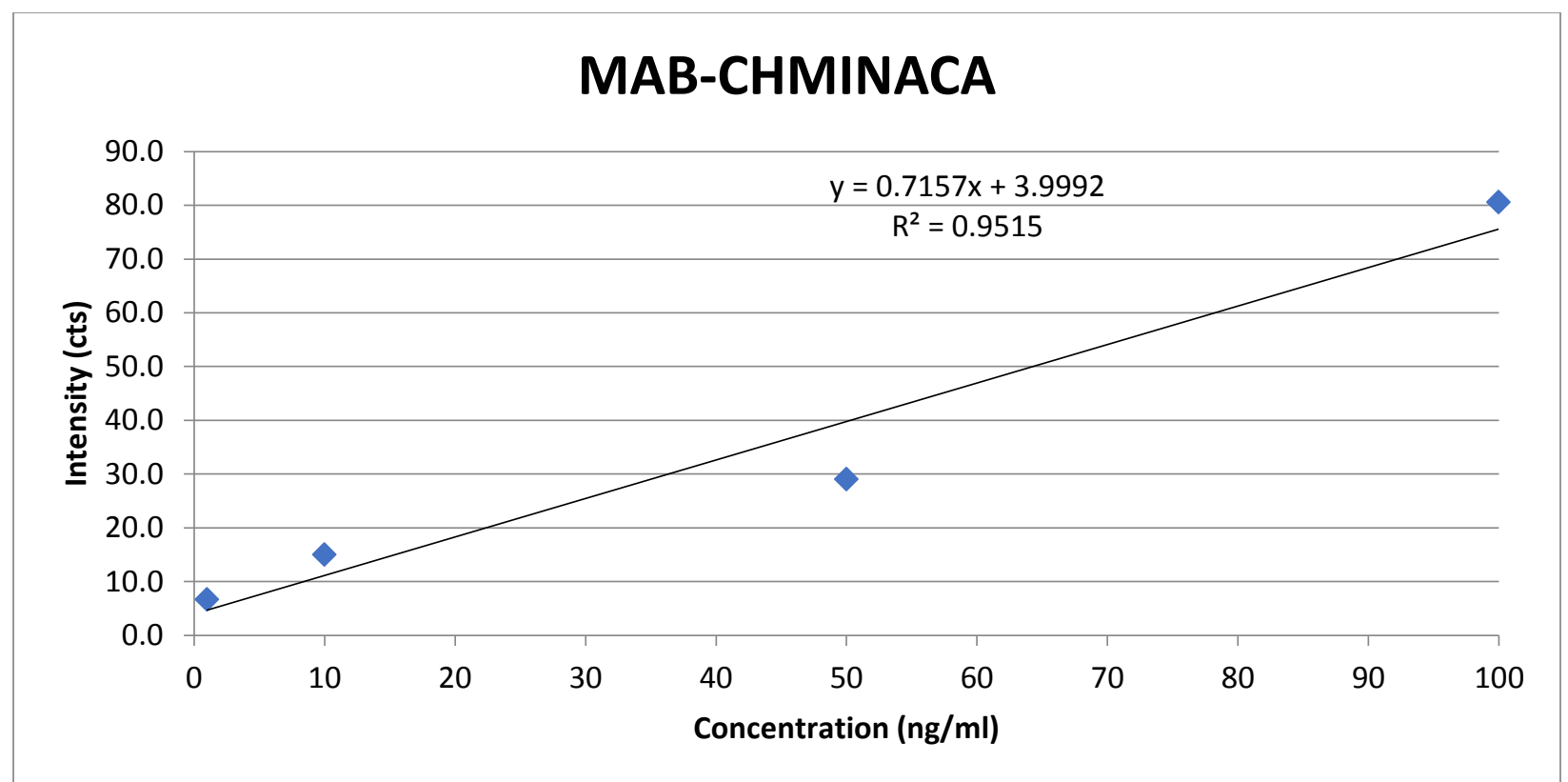

Figure E19 - Linearity plot of concentration $(1-100 \mathrm{ng} / \mathrm{ml})$ vs intensity for MAB-CHMINACA

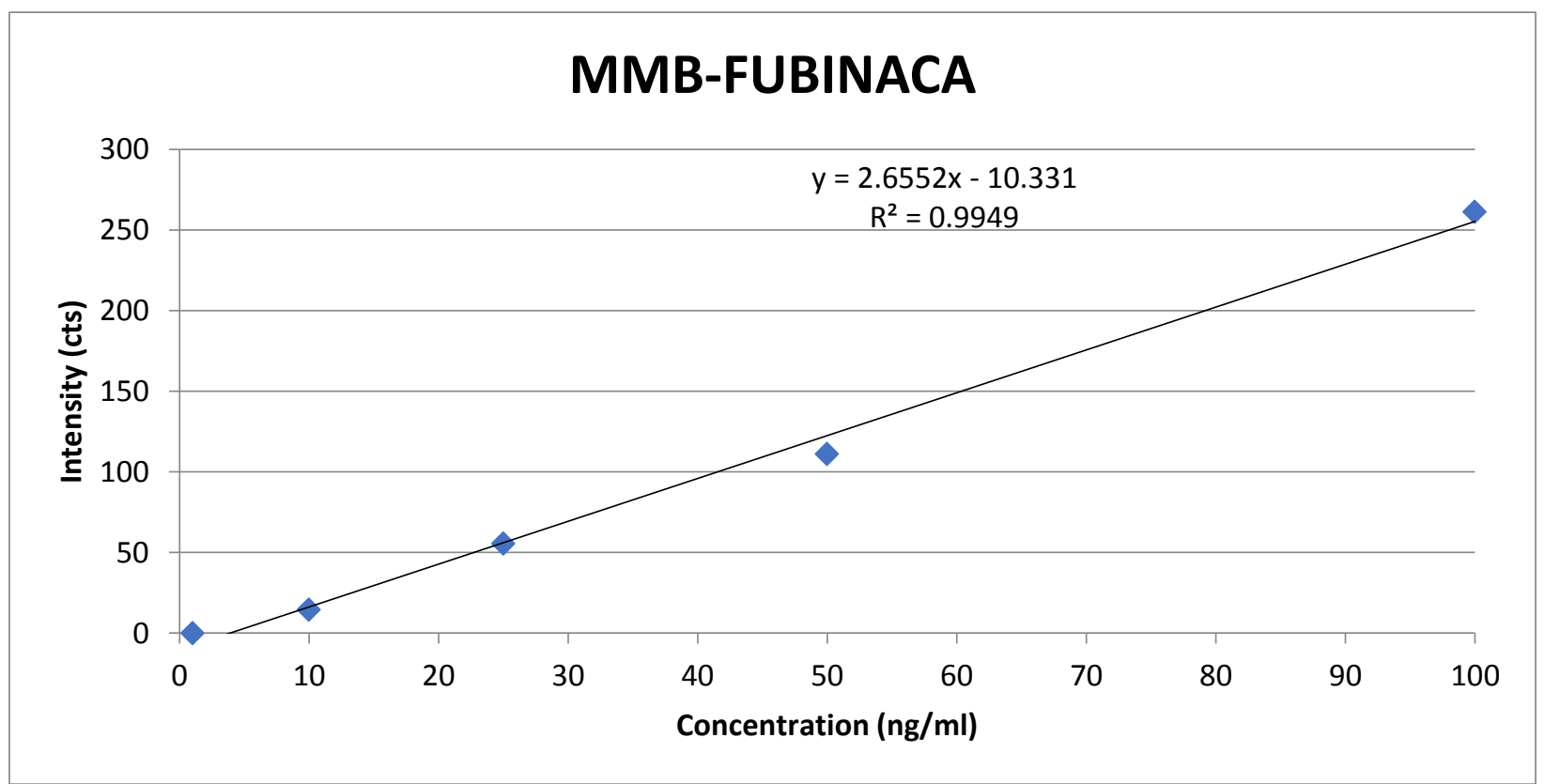

Figure E20 - Linearity plot of concentration $(1-100 \mathrm{ng} / \mathrm{ml})$ vs intensity for MMB-FUBINACA 
LC chromatograms of the parent method and examples of a few blood samples:

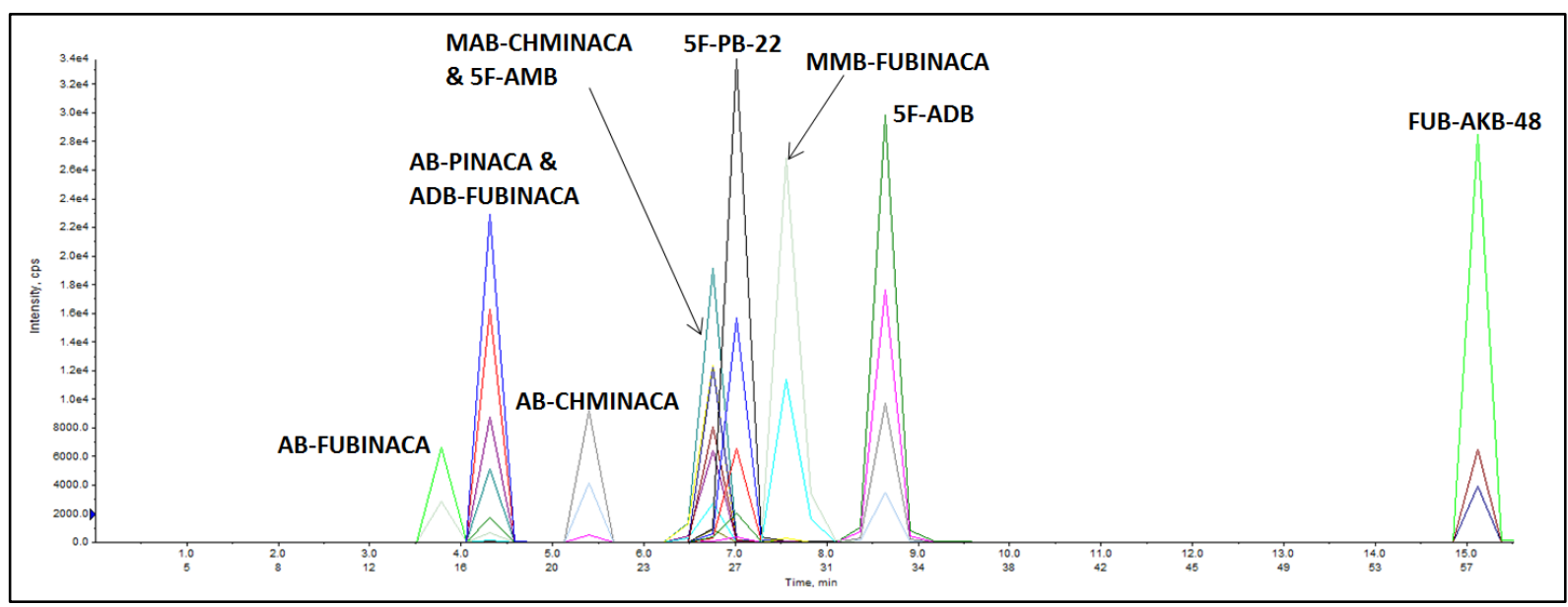

Figure E21 - LC chromatograph of a mixture of all 10 synthetic cannabinoids

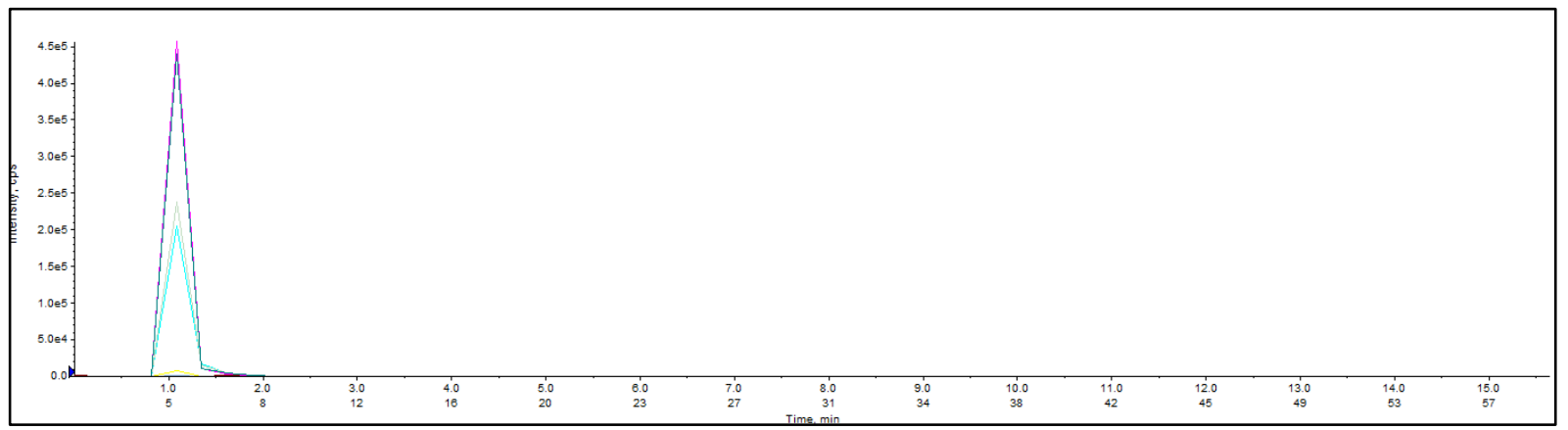

Figure E22 - LC chromatogram of a standard of 4-Hydroxycinnoline

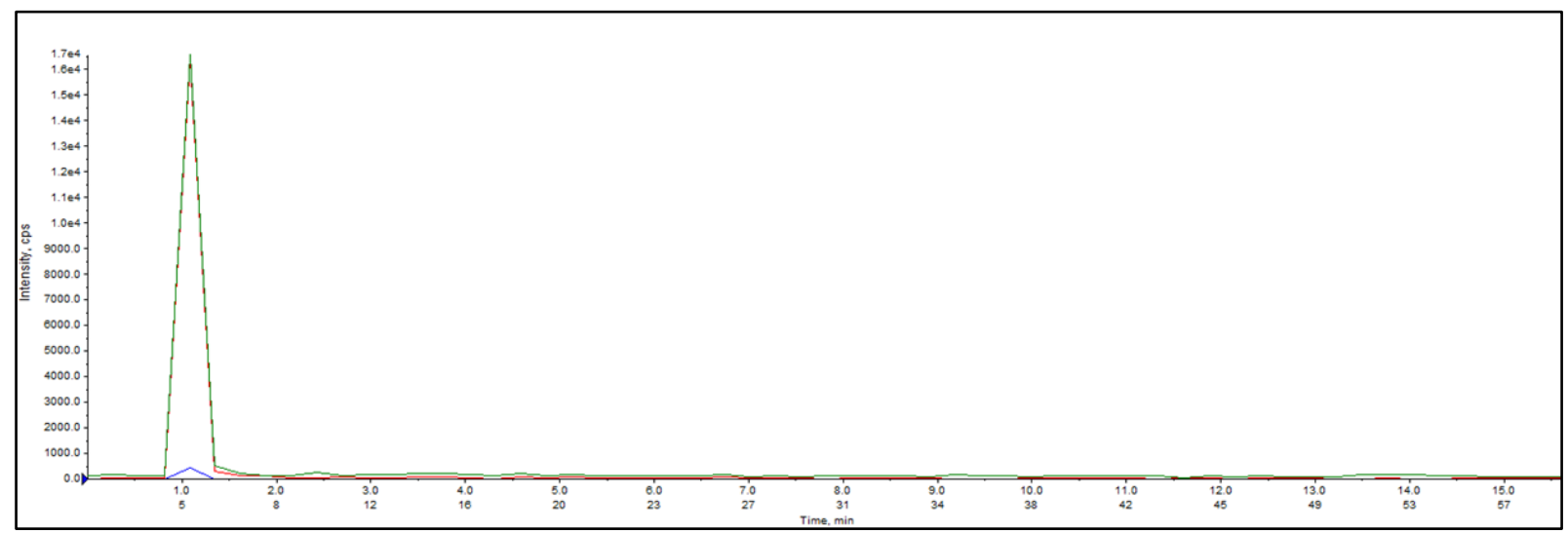

Figure E23 - LC chromatogram of a standard of 1H-Indazole-3-carboxaldehyde 


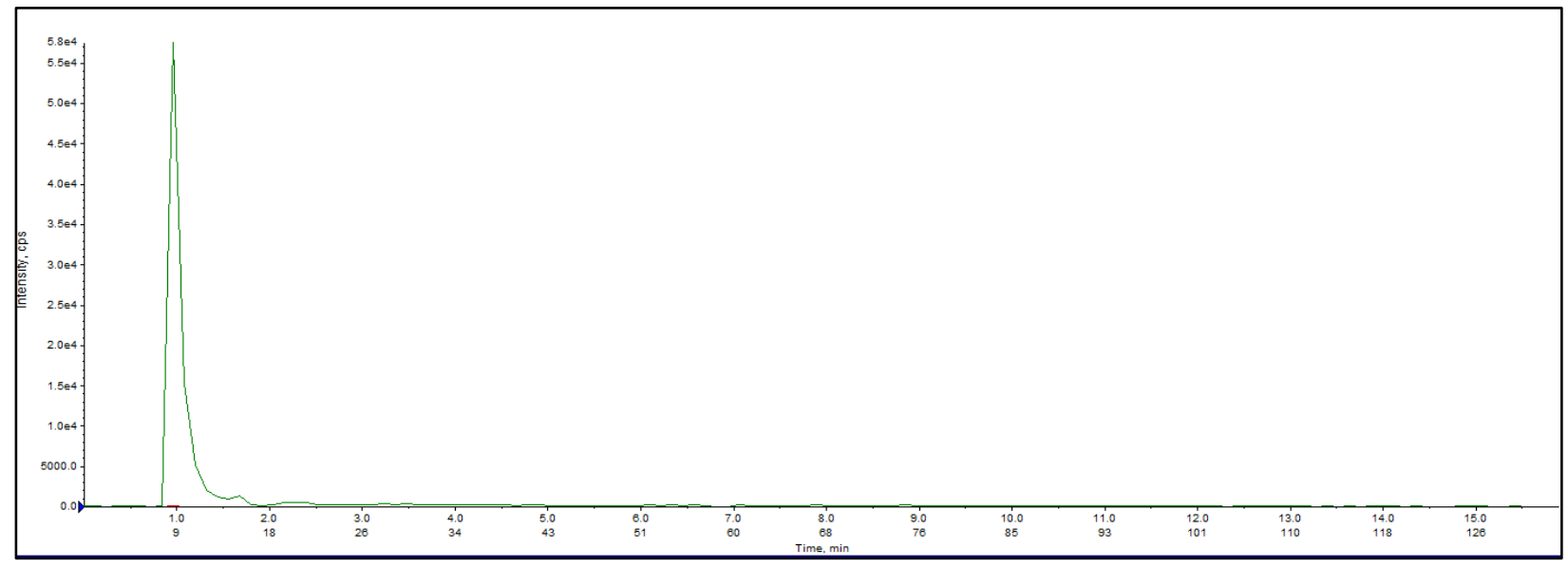

Figure E24 - LC chromatogram of a standard of Cinnoline

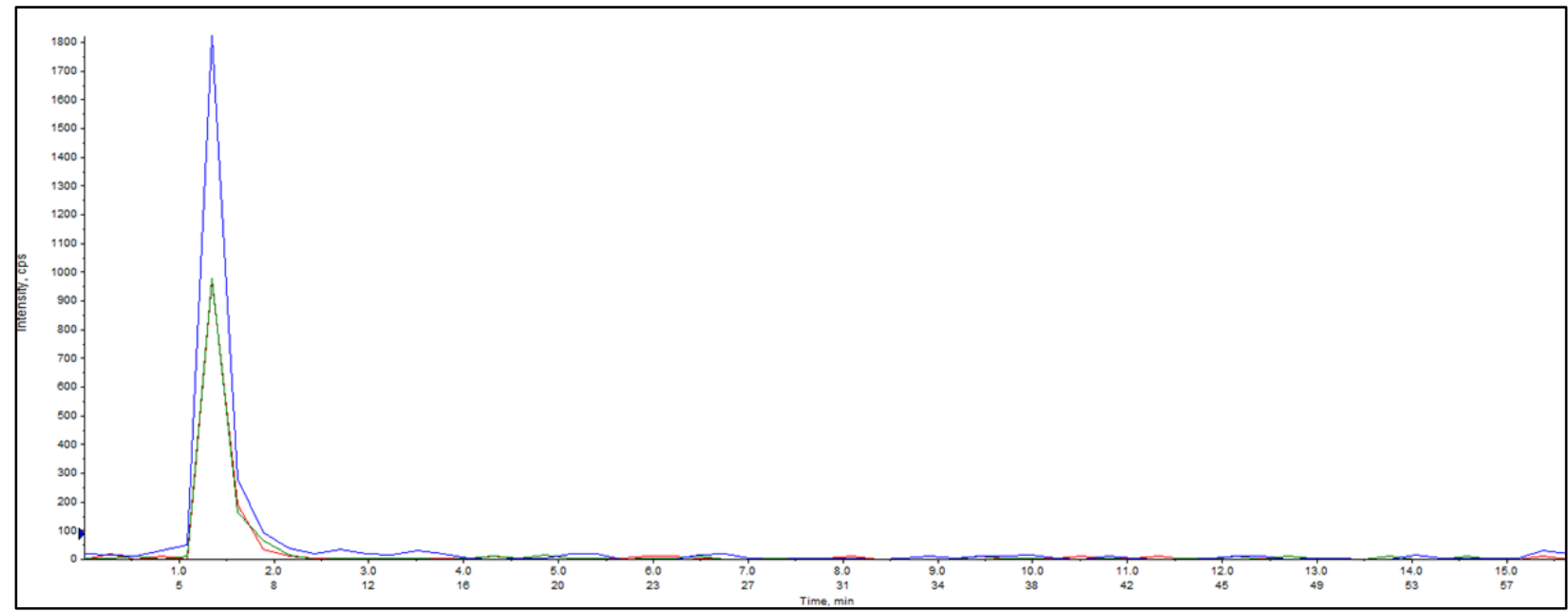

Figure E25 - LC chromatogram of a standard of 1-Methylindazole

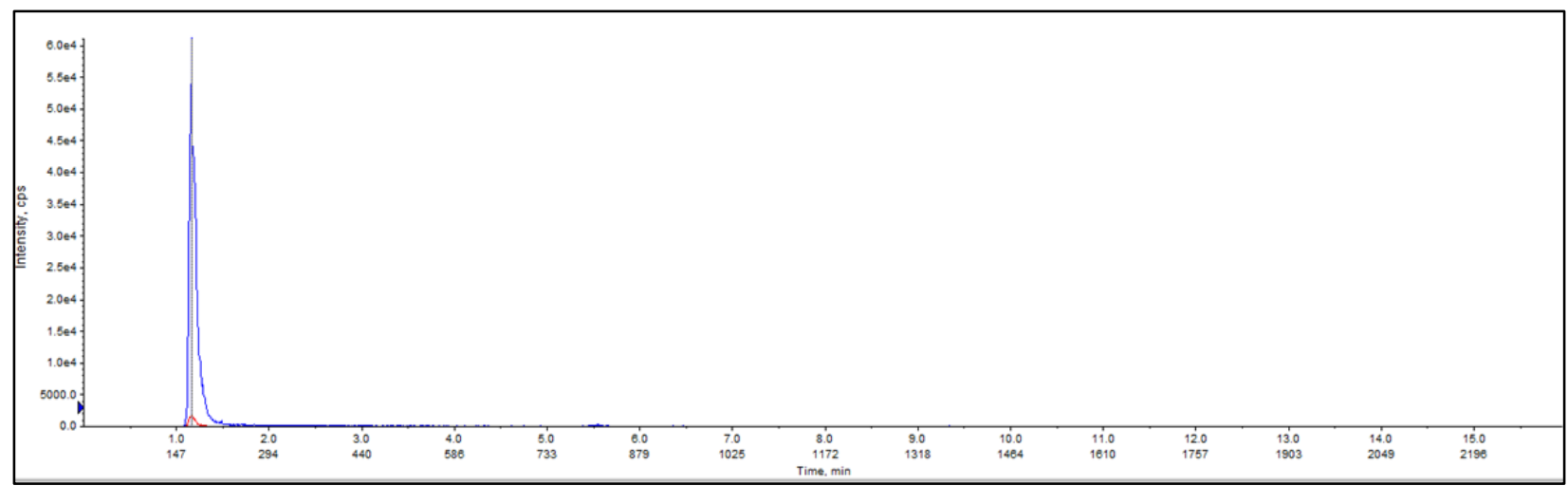

Figure E26 - LC chromatogram of a standard of Indazole 


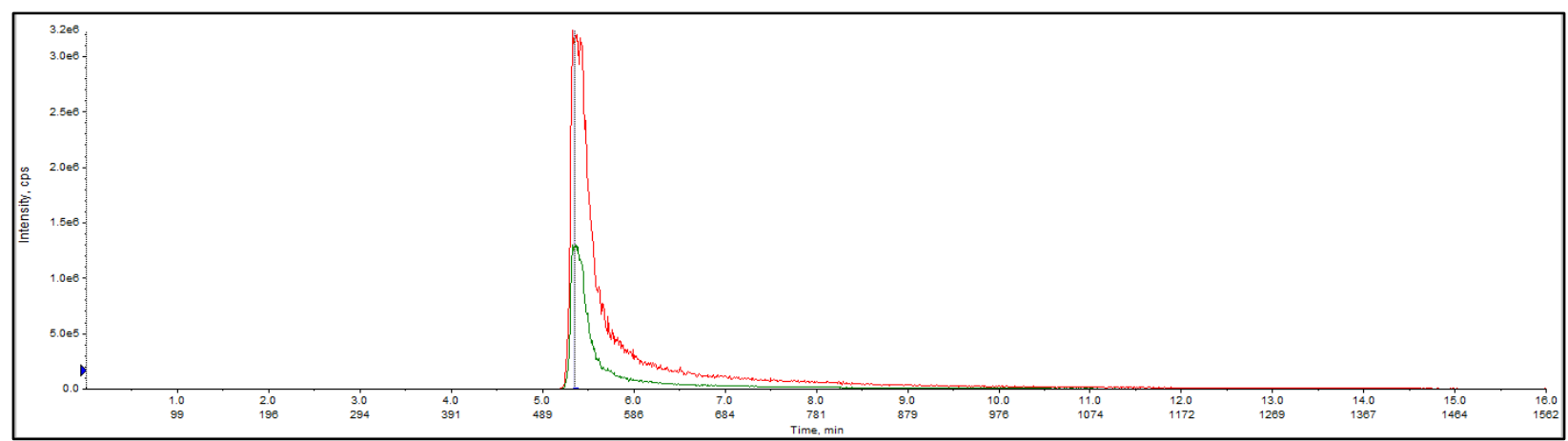

Figure E27 - LC chromatogram of a standard of 1-Pentylindazole

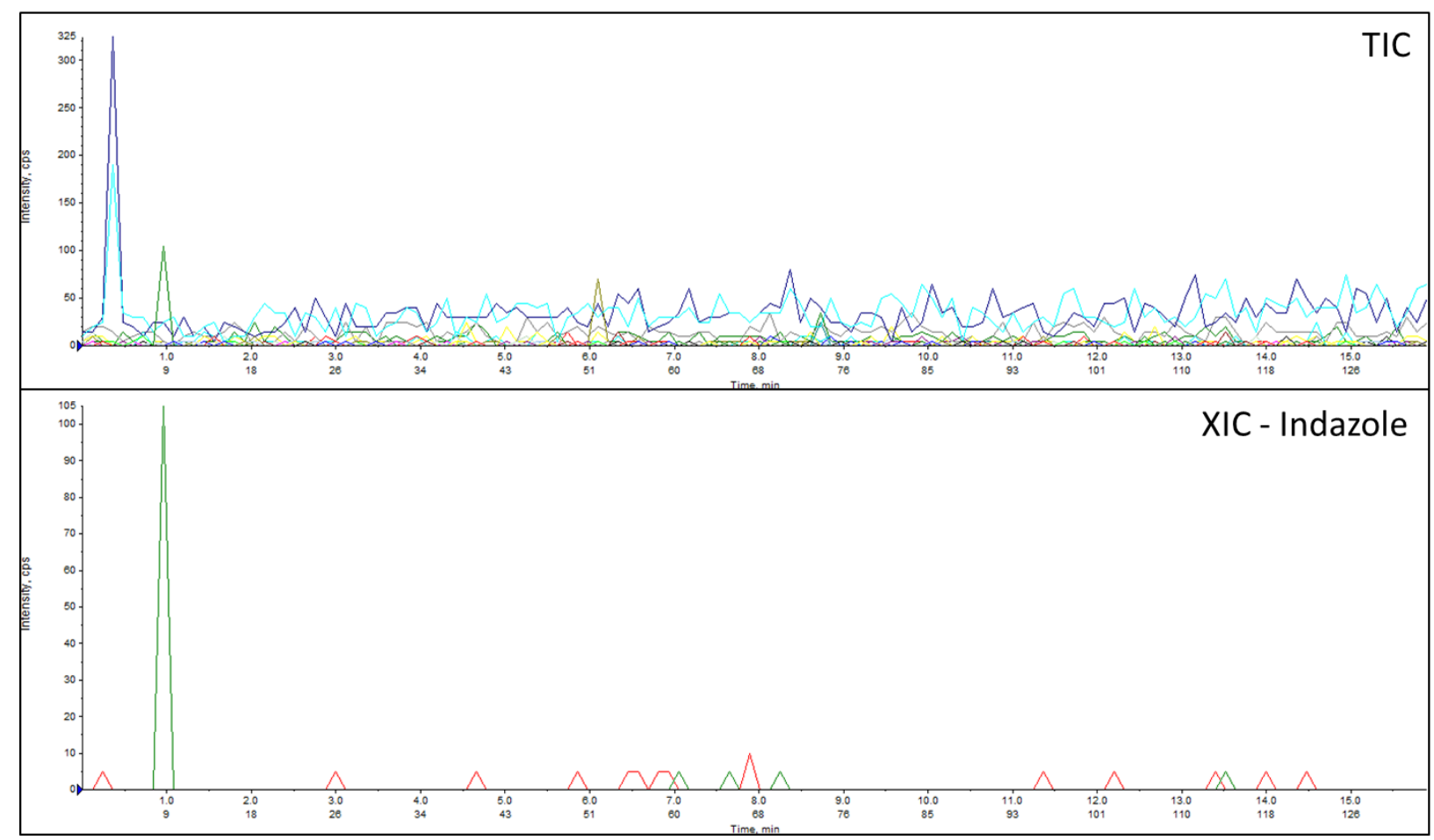

Figure E28 - Example LC chromatogram of a blood sample containing indazole 


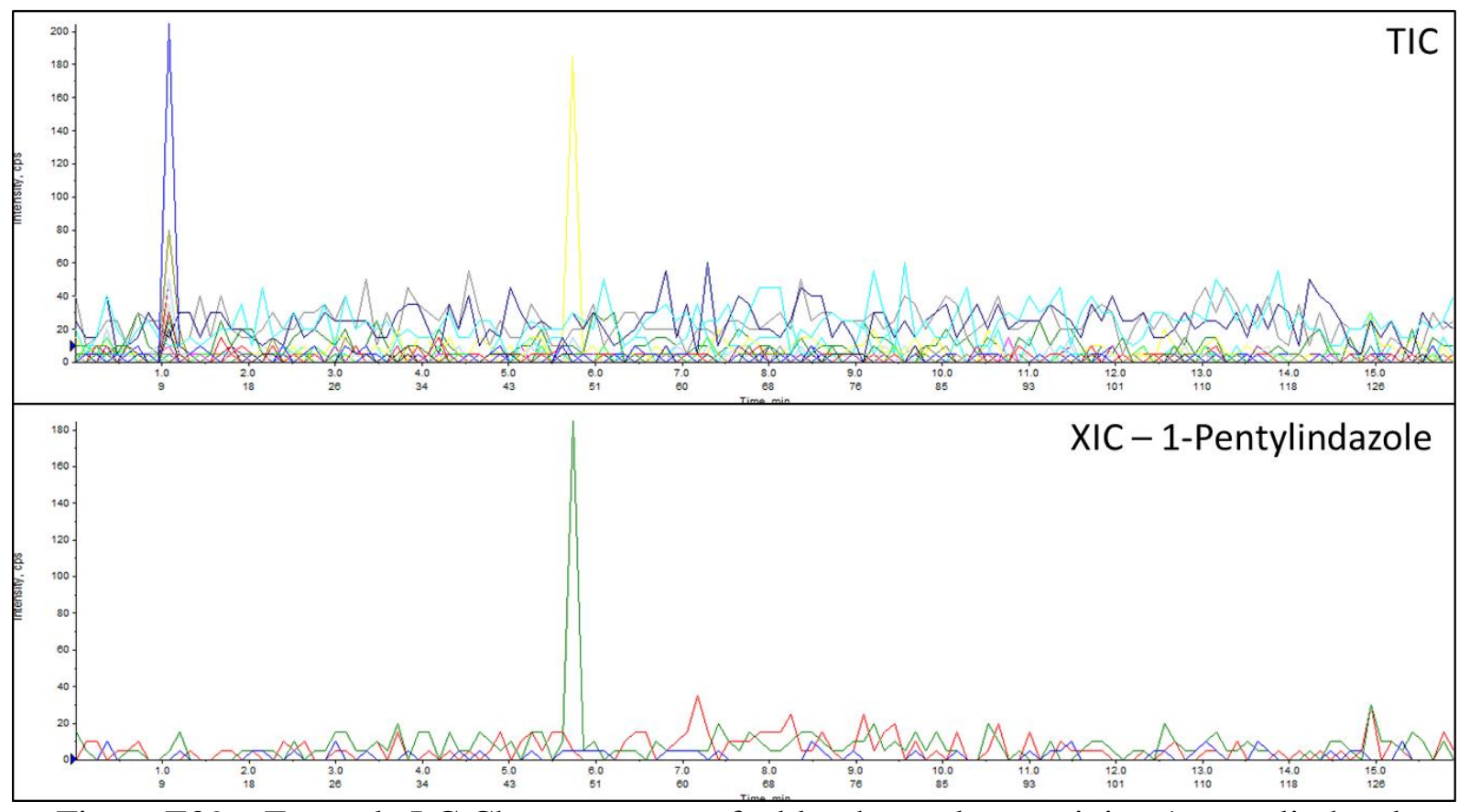

Figure E29 - Example LC Chromatogram of a blood sample containing 1-pentylindazole

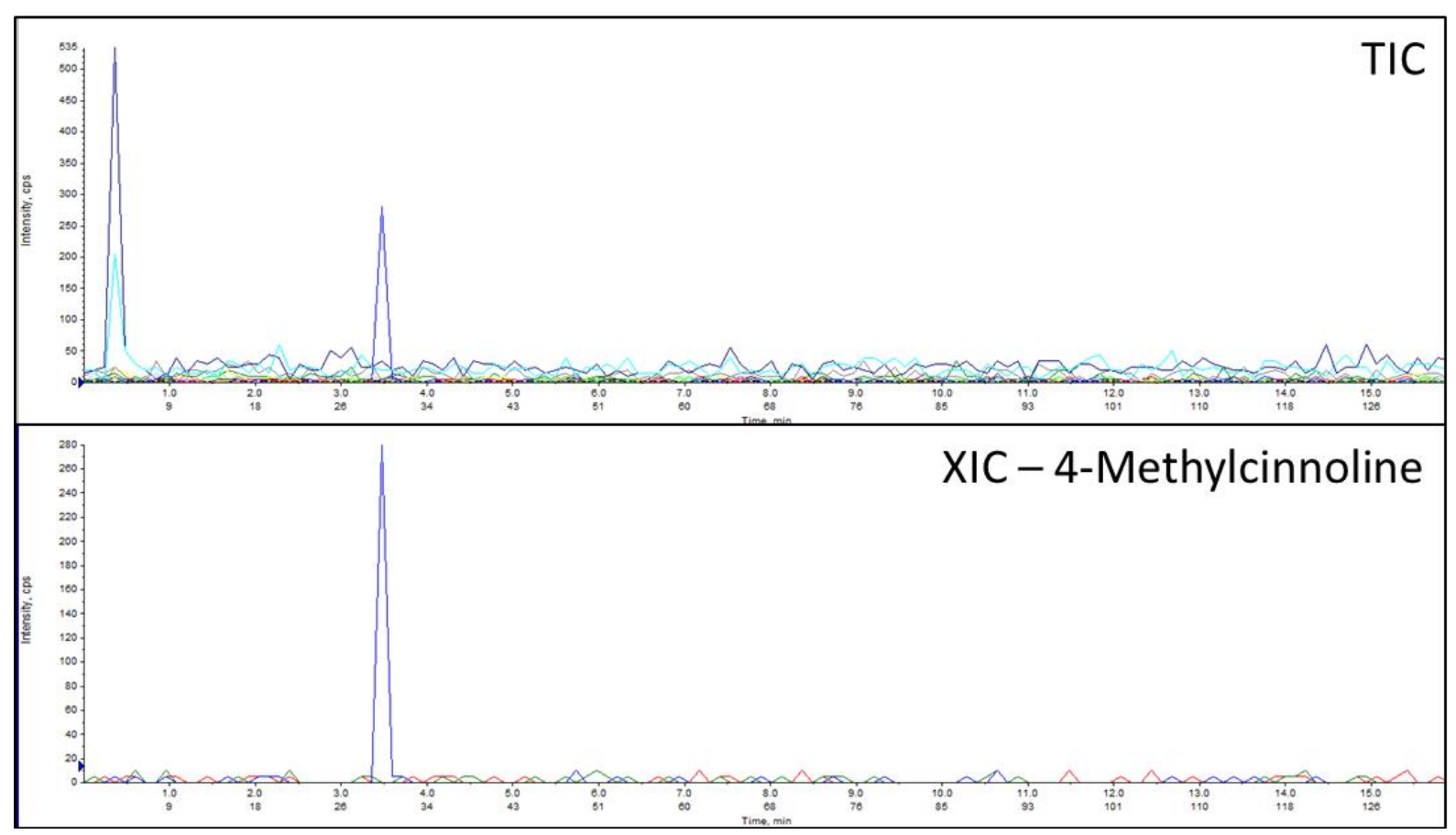

Figure E30 - Example LC Chromatogram of a blood sample containing 4-methylcinnoline 